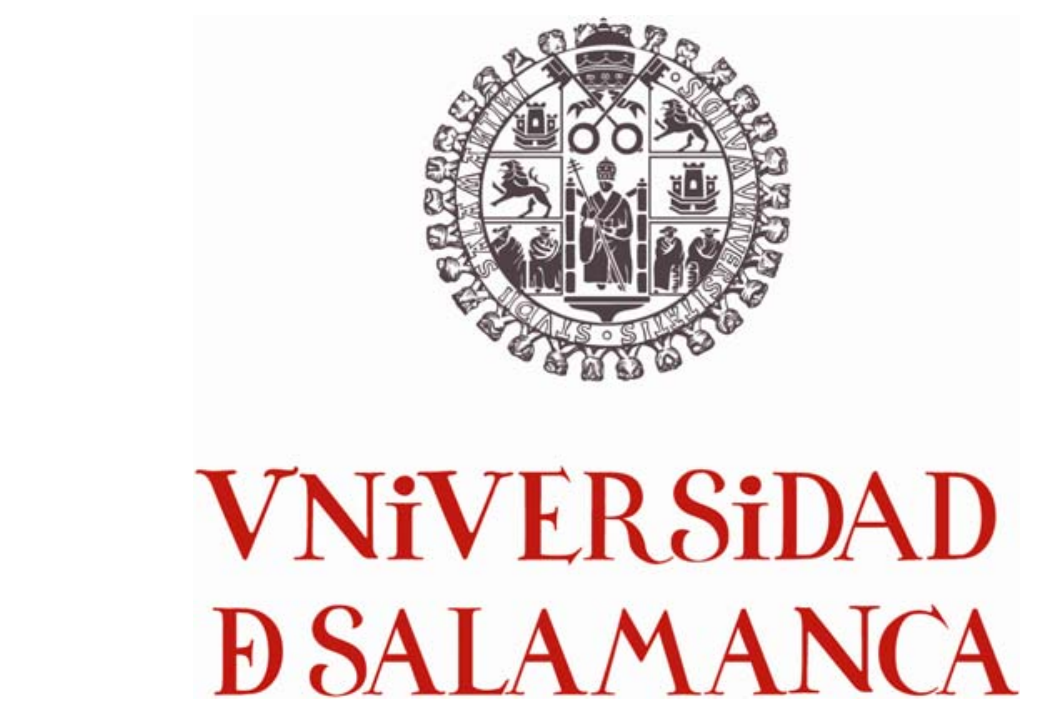

Departamento de Química I norgánica

\title{
Preparación y actividad catalítica de sistemas cromo-arcilla y níquel-arcilla
}

Guiomar Mata Ortiz

Salamanca, 2012 



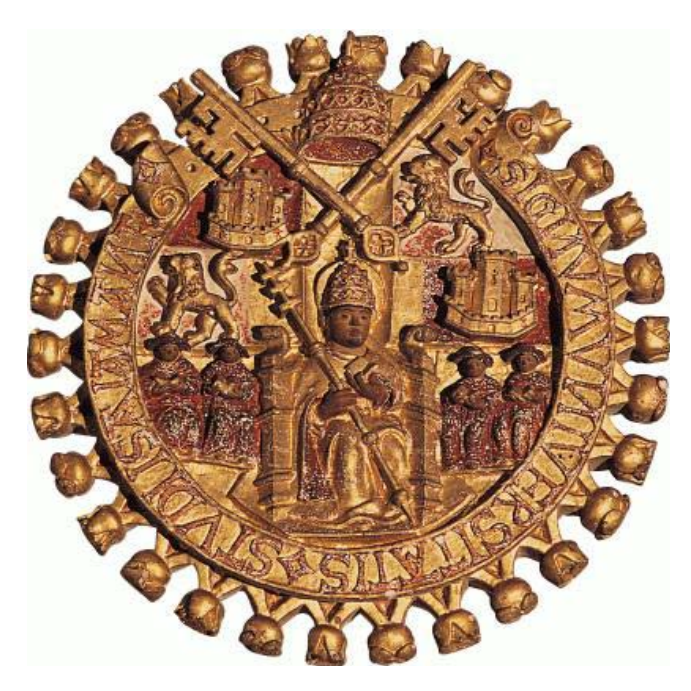

\section{Departamento de Química I norgánica}

\section{Preparación y actividad catalítica de sistemas cromo-arcilla y níquel-arcilla}

Memoria presentada por la Licenciada en Química Da Guiomar Mata Ortiz para optar al Grado de Doctora en Ciencias Químicas.

Salamanca, a 10 de Septiembre de 2012 



\section{VNiVERSIDAD D SALAMANCA}

Raquel Trujillano Hernández, Profesora Titular de Química Inorgánica de la Universidad de Salamanca, y Miguel Ángel Vicente Rodríguez, Catedrático de Química Inorgánica de la Universidad de Salamanca, como Directores del trabajo titulado "Preparación y actividad catalítica de sistemas cromo-arcilla y níquelarcilla",

\section{HACEN CONSTAR:}

Dicho trabajo ha sido realizado por la Licenciada en Química Da Guiomar Mata Ortiz para optar al Grado de Doctora en Química, dentro del Programa de Doctorado "Reactividad Química y Tecnología Medioambiental”.

Considerando que constituye un trabajo original de investigación, se encuentra concluido, y cumple todos los requisitos exigibles para su defensa pública, autorizan su presentación.

En Salamanca, a 10 de Septiembre de 2012 



\section{AGRADECI MI ENTOS}

Deseo expresar mi más sincero agradecimiento a los directores de esta Tesis Doctoral, D. Miguel Ángel Vicente y Dạ Raquel Trujillano, por proponer el tema del presente trabajo, por poner a mi disposición todo su tiempo y sus conocimientos, y por su ayuda, su apoyo y las facilidades ofrecidas en su labor de dirección durante todo este tiempo.

Quisiera agradecer a todos los miembros del Departamento de Química Inorgánica de la Universidad de Salamanca por haberme facilitado la realización de este trabajo, su ayuda y su colaboración.

A la Dra. Carolina Belver, gracias por su colaboración, su paciencia y todas sus enseñanzas.

Al Instituto de Catálisis y Petroleoquímica, por el recibimiento y las facilidades puestas a mi disposición durante mi estancia en el mismo.

A todos los compañeros del Departamento de Química Inorgánica, Mar, Christelle, Patri, Dani, Camilo, los Santis, Iván... porque todos, de una u otra forma, han contribuido a la realización de este trabajo y lo han llenado de buenos momentos dentro y fuera de los laboratorios. A Elena, Ricardo, Sole, Raquel, Emilio... porque todos habéis sido mi familia en Salamanca.

A Carlos, por condicionar su tiempo a mi estudio, por estudiar las tardes conmigo y porque su dedicación y su ayuda también merecen una Tesis.

A mis padres, a mis hermanos y mi familia, porque siempre habéis estado a mi lado y siempre habéis confiado en mí. 

Este trabajo ha sido parcialmente financiado por el Ministerio de Ciencia y Tecnología / Ministerio de Ciencia e Innovación mediante los Proyectos de Investigación con referencias MAT2002-03526, MAT2007-66439 y MAT2010-21177, y por la Junta de Castilla y León mediante los Proyectos de Investigación con referencias SA012/04, SA101A07 y SA009A11-2.

Parte de los resultados recogidos en esta Tesis Doctoral han sido obtenidos en colaboración con el Grupo de Tecnologías y Aplicaciones Medioambientales (TAMA) de la Universidad Pública de Navarra, en ocasiones mediante participación conjunta o coordinada en algunos de los Proyectos antes mencionados.

Parte de los resultados plasmados en esta Tesis Doctoral fueron obtenidos durante una estancia de investigación en el Instituto de Catálisis y Petroleoquímica del CSIC.

La colaboración con el Grupo Sol-Gel de la Universidade de Franca (Brasil) ha sido posible gracias a la financiación del Ministerio de Educación / Ministerio de Educación, Cultura y Deporte, a través del Programa Hispano-Brasileño de Cooperación Interuniversitaria, mediante los Proyectos con referencias PHB20050077 y PHB2011-0164.

La empresa TOLSA (Madrid) ha suministrado desinteresadamente las saponitas utilizadas en este trabajo, al tiempo que ha mostrado su interés en los resultados obtenidos mediante el seguimiento de algunos de los Proyectos de Investigación antes citados.

Agradecemos muy sinceramente la financiación de nuestro trabajo por parte de los organismos indicados, así como la colaboración de la empresa y los laboratorios mencionados. 

A mi madre

A mi padre

A mis hermanos

A mis ahijados 



\section{ÍNDICE}

\section{CAPÍ TULO I : I NTRODUCCIÓN, ANTECEDENTES Y OBJ ETI VOS}

I.1. Introducción $\quad 7$

$\begin{array}{ll}\text { I.2. Minerales arcillosos } & 7\end{array}$

I.3. Origen de las arcillas 8

I.4. Estructura y clasificación de las arcillas laminares 10

I.5. Propiedades físico-químicas de las arcillas 15

I.5.1. Capacidad de intercambio catiónico 16

$\begin{array}{ll}\text { I.5.2. Propiedades estructurales } & 18\end{array}$

$\begin{array}{ll}\text { I.5.3. Propiedades texturales } & 19\end{array}$

$\begin{array}{ll}\text { I.5.4. Propiedades catalíticas } & 19\end{array}$

I.6. Aplicaciones de las arcillas $\quad 21$

I.7. Características generales de la saponita 23

I.8. Intercalación y pilarización 24

I.9. Procesos catalíticos 28

I.9.1. Catalizadores soportados 29

I.10. El método sol-gel y el método de co-precipitación 33

I.11. Objetivos de este trabajo 34

I.12. Publicaciones que componen esta Tesis Doctoral 37

I.12.1. Resumen general de los resultados obtenidos 38

1.12.2. Indicios de calidad de las revistas en las que se han publicado

los resultados de esta Tesis Doctoral 43

I.13. Bibliografía 45

\section{CAPÍ TULO II: MATERI ALES DE PARTI DA Y MÉTODOS EXPERI MENTALES}

II.1. Materiales de partida $\quad 51$

II.2. Preparación de los catalizadores 53

II.2.1. Modificaciones químicas de la saponita 53

II.2.1.a. Intercalación y pilarización 53

II.2.1.b. Impregnación $\quad 57$

II.2.1.b.1. Muestras impregnadas con cromo 57

II.2.1.b.2. Muestras impregnadas con níquel $\quad 59$

II.2.2. Método sol-gel 60

II.2.3. Método de co-precipitación 62 
II.3. Métodos experimentales

II.3.1. Análisis químico $\quad 62$

II.3.2. Difracción de rayos $X$

II.3.3. Análisis térmicos 64

II.3.3.a. Análisis térmico gravimétrico 64

II.3.3.b. Análisis térmico diferencial 64

II.3.3.c. Reducción a temperatura programada 64

II.3.4. Espectroscopia Visible-Ultravioleta-Reflectancia Difusa 65

II.3.5. Espectroscopia Infrarroja por Transformada de Fourier 65

II.3.6. Isotermas de adsorción-desorción de nitrógeno a 77K 65

II.3.7. Microscopía electrónica 66

II.3.7.a. Microscopía electrónica de transmisión 66

II.3.7.b. Microscopía electrónica de barrido 66

II.4. Evaluación de la actividad catalítica 67

II.4.1. Reacción DeNOx $\quad 67$

II.4.1.a. Rectas de calibrado de reactivos y productos 68

II.4.1.b. Estudio del tamaño de partícula 69

II.4.1.c. Cálculo de la velocidad espacial $\quad 69$

II.4.1.d. Tratamiento matemático de los resultados obtenidos 70

II.4.2. Reacciones de oxidación de Z-cicloocteno y ciclohexano 71

II.5. Reactivos utilizados

II.6. Bibliografía 75

CAPÍTULO III. CHROMIUM-SAPONITE CLAY CATALYSTS: PREPARATION, CHARACTERIZACION AND CATALYTI C PERFORMANCE IN PROPENE OXIDATION

$\begin{array}{ll}\text { Abstract } & 79\end{array}$

III.1. Introduction $\quad 79$

III.2. Experimental 81

III.2.1. Raw materials and preparation of the catalysts 81

III.2.2. Characterization techniques 83

III.3. Results and discussion 84

III.3.1. Characterization of the catalysts $\quad 84$

$\begin{array}{ll}\text { III.3.2. Catalytic performance } & 97\end{array}$

$\begin{array}{ll}\text { III.4. Conclusions } & 101\end{array}$

$\begin{array}{ll}\text { III.5. References } & 101\end{array}$ 
CAPÍtUlo IV. estudio de LA PREPARACIÓN DE CATALIZADORES CROMOSAPONITA PILAREADA Y DE SU COMPORTAMIENTO CATALÍtICO EN LA OXI DACIÓN DE PROPENO

Abstract

IV.1. Introducción

IV.2. Experimental

IV.3. Técnicas de caracterización

IV.4. Caracterización de los sólidos

IV.4.1. Sólidos intercalados y pilareados

IV.4.2 Sólidos impregnados

IV.5. Actividad catalítica

IV.6. Conclusiones

IV.7. Bibliografía

CAPÍtUlo V. NI-PILLARED CLAyS AS CATALySTS FOR THE SELECTIVE CATALYTI C REDUCTI ON OF NITROGEN OXI DES

Abstract

V.1. Introduction

V.2. Experimental

V.2.1. The starting clay

V.2.2. Synthesis of $\mathrm{Ni}$-clay catalysts

V.2.3. Characterization of the solids

V.2.4. HC-SCR catalytic activity

V.3. Results and discussion

V.3.1. Preparation and Characterization of Ni-clays

V.3.2. Catalytic properties: Selective catalytic reduction of nitrogen oxides 


\section{CAPÍtULO VI. (Z)-CYCLOOCTENE EPOXIDATION AND CYCLOHEXANE OXI DATION ON NI/ ALUMI NA-PI LLARED CLAY CATALYSTS}

Abstract

VI.1. Introduction

VI.2. Experimental

VI.2.1. Raw materials, intercalation process and catalysts preparation

VI.2.2. Characterization techniques

VI.2.3. Catalytic performance

VI.3. Results and discussion

$\begin{array}{ll}\text { VI.3.1. Characterization of the catalysts } & 148\end{array}$

VI.3.2. Catalytic performance 155

VI.4. Conclusions 163

$\begin{array}{ll}\text { VI.5. References } & 163\end{array}$

CAPÍtULO VII. PREPARATION AND CHARACTERIZATION OF NEW NIALUMINOSIllicate CATALYSTS AND THEIR PERFORMANCE IN THE EPOXI DATION OF (Z)-CYCLOOCTENE

Abstract

VII.1. Introduction

VII.2. Experimental

VII.2.1. Preparation of the solids

VII.2.2. Characterization techniques

VII.2.3. Catalytic performance

VII.3. Results and discussion

VII.3.1. Chemical analysis

VII.3.2. X-Ray diffraction

VII.3.3. Thermal analyses

VII.3.4. UV-Vis spectroscopy

VII.3.5. Textural properties

VII.3.6. Catalytic performance

VII.4. Conclusions

\section{CAPÍTULO VIII. CONCLUSI ONES}

VIII.1. Materiales de partida

VIII.2. Propiedades de los catalizadores utilizados 
Capítulo I

I ntroducción, antecedentes objetivos 



\section{I.1. Introducción}

Los minerales arcillosos son materiales abundantes en la naturaleza y con una gran diversidad de propiedades y aplicaciones. Consecuentemente, han sido utilizados por el hombre desde los albores de la Humanidad, en forma de utensilios cotidianos, expresiones artísticas, escritura, construcción, etc.

Teniendo en cuenta esta amplia relación del hombre con los minerales arcillosos, no resulta extraño que se vuelva la vista hacia estos minerales cada vez que surge una nueva aplicación tecnológica. Su versatilidad hace que, con frecuencia, consigan adaptarse a estas nuevas aplicaciones, en ocasiones tras los oportunos tratamientos físico-químicos. Por citar solo algunos ejemplos recientes, podemos mencionar la preparación de nuevos adsorbentes, catalizadores y materiales híbridos orgánico-inorgánicos. Todo esto, unido a la abundancia de estos minerales en la corteza terrestre, su bajo coste y su distribución en todos los países, hace que el estudio de sus propiedades, y de los métodos para mejorarlas, continúe siendo objeto de numerosas investigaciones.

\section{I.2. Minerales arcillosos}

Es más correcto utilizar el término "mineral arcilloso" que "arcilla", aunque en nuestro campo de trabajo se consideren con frecuencia sinónimos. Estos términos se utilizan de manera general para designar productos resultantes de la meteorización, productos alterados hidrotermalmente y depósitos sedimentarios. Así, pueden definirse las arcillas como rocas sedimentarias, con frecuencia fragmentables, que, empapadas de agua, pueden formar una masa más o menos plástica. Este término designa igualmente a una categoría de minerales, los filosilicatos, que entran, en gran proporción, en la constitución de las rocas arcillosas. Además, este término tiene otra acepción relacionada con el tamaño de partícula; la fracción formada por partículas de tamaños más pequeños se denomina fracción arcillosa. Normalmente, los científicos que trabajan con arcillas consideran el límite de dos micras como el superior para el tamaño de partícula de los minerales arcillosos. Atendiendo a esta definición de materiales arcillosos, que se refiere más al tamaño de partícula que a criterios mineralógicos, pueden considerarse arcillas a minerales que, aun no perteneciendo al grupo de los 
filosilicatos, estén presentes en los sedimentos y su tamaño de partícula no supere las dos micras $[1,2]$.

El aspecto morfológico de las arcillas es visible únicamente al microscopio electrónico. Su identificación se efectúa por difracción de rayos $X$, y su caracterización puede completarse mediante análisis químicos y térmicos, entre otros. Aplicando la difracción de rayos $X$ a la caracterización de las arcillas se encuentra que, desde el punto de vista cristalográfico, existen dos grandes categorías de minerales arcillosos: las arcillas de estructura foliar o laminar y las arcillas de estructura fibrosa. Las arcillas de estructura laminar son las más habituales, están formadas por la superposición de láminas, y cada lámina a su vez está constituida por varias capas. Al ser las arcillas utilizadas en este trabajo, su estructura y composición serán ampliamente discutidas a continuación (Apartado 1.4). Por su parte, las arcillas fibrosas están formadas por estructuras en bandas y comprenden dos categorías principales, las paligorskitas (con composición alumínico-magnésica) y las sepiolitas (con composición esencialmente magnésica).

\section{I.3. Origen de las arcillas}

Las arcillas se originan en la naturaleza debido a las interacciones de las rocas con el agua que hidrata los silicatos presentes en ellas. Las diferentes condiciones en las que puede tener lugar la interacción, así como las distintas proporciones de agua y roca que interaccionan, dan lugar a reacciones diferentes y, por tanto, a distintos productos de reacción. Si la interacción se produce con una elevada cantidad de agua, la disolución de la roca es total, mientras que si la cantidad es menor, se producirá una disolución parcial.

La precipitación directa en zonas húmedas, como zonas pantanosas y márgenes fluviales, y los principales procesos geológicos, diagénesis, meteorización, deposición y alteración hidrotermal, pueden dar lugar a la formación de arcillas $[2,3]$.

La diagénesis se produce en depósitos donde se han acumulado varias capas de minerales arcillosos. La deposición consecutiva capa sobre capa de sedimentos hace que cada capa vaya siendo acumulada y enterrada de forma progresiva, lo que aumenta la temperatura y la presión sobre las capas inferiores. En estas 
condiciones, los materiales de las capas inferiores se vuelven más compactos y la relación agua/arcilla disminuye drásticamente, hasta que el agua está en cantidades mínimas. De esta forma, y con un alto grado de compactación, las interacciones que se producen son casi exclusivamente arcilla-arcilla [4].

El proceso de meteorización incluye procesos físicos y químicos. La meteorización física, o mecánica, produce una ruptura de las rocas por esfuerzos internos o externos, lo que implica su fragmentación en trozos más o menos grandes y angulosos, pero sin modificación alguna en la naturaleza mineralógica de la roca. Los procesos más importantes de la meteorización física son la temoclastia, la gelificación, la hidroclasia, la haloclasia y la corrosión. Por su parte, la meteorización química produce modificaciones en la naturaleza de las rocas originarias, tales como oxidación, hidratación, hidrólisis o carbonatación [2].

Mediante los procesos de sedimentación o deposición, las arcillas procedentes de la meteorización son transportadas y depositadas en otros lugares. Durante este transporte las arcillas pueden reaccionar con la disolución en la que son transportadas, o con otros elementos presentes en ella. Se pueden producir reacciones de intercambio catiónico con elementos presentes en la disolución transportadora o interacciones arcilla-arcilla. La mayoría de las arcillas de origen sedimentario se encuentran en depósitos de márgenes fluviales, océanos o lagos.

Las modificaciones hidrotermales de las arcillas consisten en la interacción de las rocas con grandes cantidades de agua caliente, generalmente de origen magmático. Estas alteraciones suelen tener lugar a temperaturas elevadas,

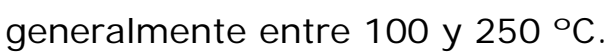

La mayor parte de los minerales arcillosos naturales tienen su origen en procesos de meteorización. Mediante diagénesis y sedimentación se producen las transformaciones de la composición química original de los sólidos o de unas arcillas en otras, mientras que los procesos hidrotermales producen arcillas de elevada pureza, muy utilizadas en la industria. 


\section{I.4. Estructura y clasificación de las arcillas laminares}

Las arcillas laminares están formadas por silicatos de aluminio, magnesio o hierro, en diferentes grados de hidratación y pueden contener cantidades muy variables de cationes alcalinos y alcalinotérreos. Pertenecen al grupo de los filosilicatos y se caracterizan por su estructura laminar, por lo que se les conoce como silicatos laminares. Cada una de las láminas que forman la estructura está a su vez constituida por capas tetraédricas y octaédricas, el empaquetamiento repetido de las láminas da lugar a las unidades estructurales [4].

La composición general de la capa tetraédrica puede expresarse como $\mathrm{T}_{2} \mathrm{O}_{5}$, donde $\mathrm{T}$ representa a los cationes $\mathrm{Si}^{4+} \circ \mathrm{Al}^{3+}$. Estos cationes se coordinan tetraédricamente con aniones oxo, $\mathrm{O}^{2-}$, y los tetraedros se unen entre sí compartiendo todos los vértices de sus bases, situando todas las bases en un mismo plano y formando anillos hexagonales, mientras que todos los vértices opuestos forman otro plano que se dirige a la capa octaédrica. De esta forma, los tres aniones compartidos se conocen como oxígenos basales, el cuarto, no compartido y situado en un plano paralelo, se denomina oxígeno apical (ver Figura I.1). Si el átomo central del tetraedro es silicio, la estructura tetraédrica se mantiene eléctricamente neutra ya que el silicio interior aporta cuatro cargas positivas y los oxígenos, al compartirse con otros tetraedros (en el plano basal) y octaedros (plano apical) aportan sólo una de sus dos cargas, con lo que tendremos en los vértices cuatro cargas negativas.

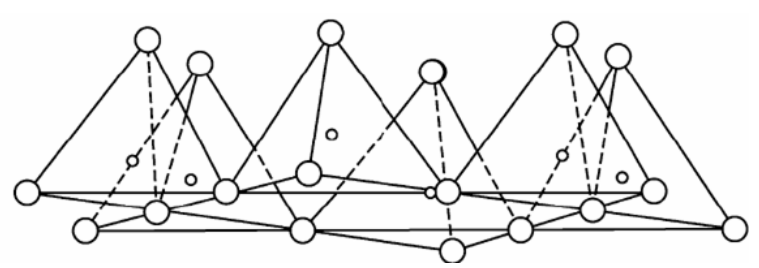

Figura I.1. Combinación de los tetraedros para formar una capa tetraédrica.

Las capas octaédricas están constituidas por dos planos paralelos de aniones $\mathrm{OH}^{-}$, entre los que se encuentran las posiciones octaédricas. Estas posiciones pueden ser ocupadas por cationes divalentes, como el $\mathrm{Mg}^{2+}$ y el $\mathrm{Fe}^{2+}$, o por cationes trivalentes, como el $\mathrm{Al}^{3+}$ o el $\mathrm{Fe}^{3+}$. El octaedro es regular. Al formar la estructura, el octaedro se apoya sobre una de las caras triangulares, con el eje cuaternario 
principal inclinado, como se representa en la Figura I.2. Los huecos octaédricos quedan en el plano central y son ocupados por los cationes. Si se trata de cationes divalentes sólo se alcanza la electroneutralidad si se ocupan todas las posiciones, en cuyo caso la capa es llamada trioctaédrica. Pero si hay cationes octaédricos trivalentes, al poseer mayor carga, dejan posiciones vacantes en la red, y en el caso extremo de que todos los cationes octaédricos sean trivalentes, se ocupan dos de cada tres posiciones posibles, y esa capa se denomina dioctaédrica [2]. ${ }^{1}$

Al igual que en las capas tetraédricas, las octaédricas ideales también son eléctricamente neutras. De las dos cargas de los aniones oxo de los vértices sólo una se comparte con el catión octaédrico, la otra carga, como se ha comentado anteriormente, se comparte con el silicio tetraédrico de la capa inferior $y$, si no se une a este catión, en ese vértice en lugar de haber un anión oxo hay un grupo hidroxilo (por ejemplo, en el borde de las partículas). Si el catión octaédrico es divalente, los vértices del octaedro deben compensar un total de dos cargas negativas y para ello cada vértice se comparte entre tres cationes $\mathrm{Mg}^{2+} \circ \mathrm{Fe}^{2+}$ (pertenece a tres octaedros). De esta manera, cada vértice aporta un tercio de carga y, como hay seis vértices, tendremos un total de dos cargas negativas por octaedro. En el caso de $\mathrm{Al}^{3+}$ y $\mathrm{Fe}^{3+}$ se necesitan tres cargas negativas en el octaedro, para ello ahora los vértices se comparten entre sólo dos octaedros. Esta compensación de cargas justifica igualmente la ocupación total de los huecos octaédricos si los cationes son divalentes (arcillas trioctaédricas), y la ocupación de dos de cada tres posiciones si los cationes son trivalentes (arcillas dioctaédricas) [1].

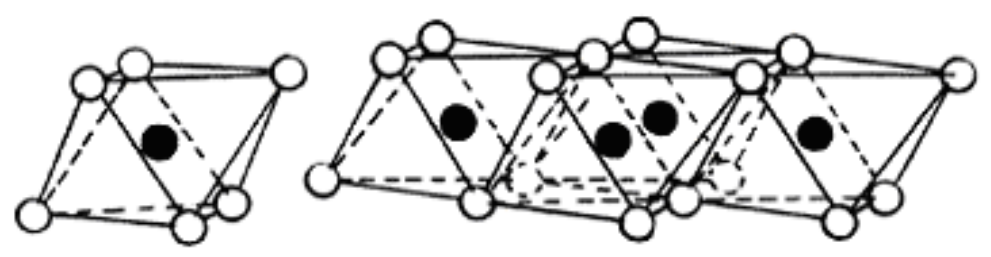

Figura I.2. Unidades octaédricas y uniones entre ellas.

\footnotetext{
${ }^{1}$ La denominación dioctaédrica-trioctaédrica para referirse a la ocupación de dos terceras partes o de la totalidad de las tres posiciones octaédricas existentes está referida a la fórmula estructural de la semicelda unidad ( 3 posiciones octaédricas, 22 cargas negativas). No obstante, la fórmula estructural suele referirse a la totalidad de la celda, en la que lógicamente se encuentran 6 posiciones octaédricas.
} 
Como se ha indicado, las capas tetraédricas y octaédricas se unen a través de los oxígenos apicales de la capa tetraédrica, que a su vez son uno de los vértices de la capa octaédrica. La combinación sucesiva de las capas da lugar a la formación de las láminas que forman la estructura laminar final de estos minerales. Estas láminas pueden estar formadas por una capa tetraédrica y una octaédrica, o bien por una octaédrica rodeada por dos tetraédricas, y se conocen como estructuras TO y TOT, o láminas $1: 1$ y 2:1, respectivamente (Figura I.3). Esta nomenclatura indica el número de capas de cada tipo presente en cada lámina.
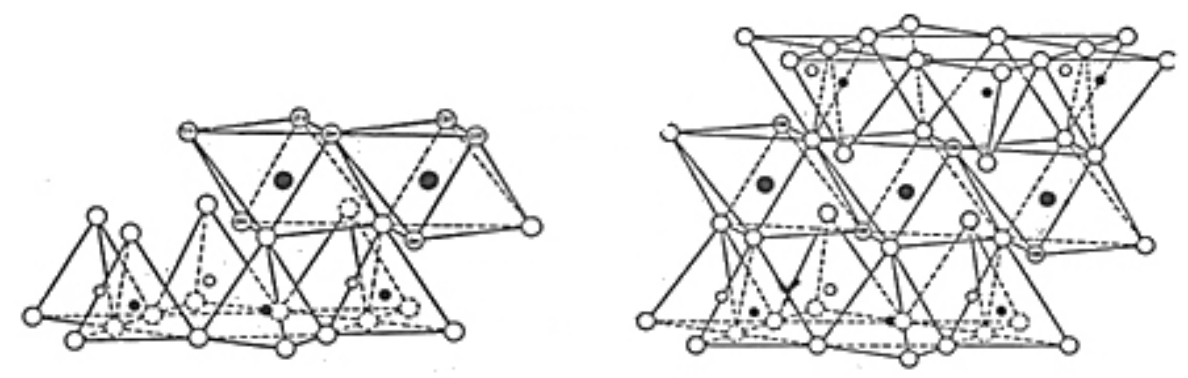

Figura I.3. Uniones TO y TOT entre capas tetraédricas y octaédricas.

Las láminas con estructura TO dan lugar a tres planos de aniones. Una de las caras de la lámina queda constituida por los oxígenos basales de la capa tetraédrica, mientras que la otra la constituyen los hidroxilos de la capa octaédrica. El plano intermedio entre los anteriores está formado por ambos tipos de aniones, $\mathrm{OH}^{-}$de la capa octaédrica y $\mathrm{O}^{2-}$ procedentes de los oxígenos apicales de la capa tetraédrica. Las estructuras TOT contienen cuatro planos de aniones, las dos caras exteriores están formadas por los oxígenos basales de las capas tetraédricas y las dos centrales por hidroxilos de la capa octaédrica y por los oxígenos apicales de la tetraédrica.

Las direcciones cristalográficas a y b se definen dentro de la lámina, tanto en las estructuras TO como en las TOT. Las láminas se apilan entre sí a lo largo de la dirección c, dando lugar a la formación de partículas, habitualmente denominadas tactoides, constituidas por ocho o diez láminas elementales superpuestas. Entre las láminas se sitúan los denominados cationes de cambio, que compensan la carga negativa generada por las sustituciones isomórficas y por las vacantes catiónicas en la lámina. 
Las diferentes distribuciones de capas dan lugar a la existencia de una gran diversidad de filosilicatos, teniendo en cuenta, además, las distintas composiciones químicas que pueden presentar y que dependen, fundamentalmente, del origen del mineral. La variedad de composiciones se debe también a la posibilidad de sustituciones isomórficas que pueden producirse tanto en las capas tetraédricas como en las octaédricas. En la capa tetraédrica, el $\mathrm{Si}^{4+}$ puede ser sustituido por $\mathrm{Al}^{3+}$ $y$, en menor medida, por $\mathrm{Fe}^{3+}$ (incluso, algunos autores sostienen que la sustitución por $\mathrm{Fe}$ es inviable). Los cationes $\mathrm{Mg}^{2+}$ y $\mathrm{Al}^{3+}$ de la capa octaédrica pueden ser sustituidos por $\mathrm{Fe}^{2+}, \mathrm{Fe}^{3+}, \mathrm{Mn}^{2+}$ y $\mathrm{Ti}^{4+}$. En esta capa, además, es frecuente que queden posiciones vacantes. Tanto las sustituciones isomórficas como las vacantes producen un defecto de carga en la lámina, para compensarlo y conseguir un balance nulo, se sitúan en la interlámina iones alcalinos, alcalinotérreos, protones o grupos amonio.

La arcilla utilizada en el presente trabajo ha sido la saponita, perteneciente al grupo de las esmectitas, filosilicatos 2:1 constituidos por una capa octaédrica magnésica, situada entre dos capas tetraédricas de sílice. Estructuralmente, la saponita es análoga a la arcilla más representativa de este grupo, que es la montmorillonita. La manera más sencilla de describir su estructura es el modelo representado en la Figura 1.4, propuesto por Hofmann et al. [5]. Esta estructura consta de una capa inferior de tetraedros de sílice que comparten los vértices de la base, mientras que el vértice opuesto está dirigido hacia la capa octaédrica. En la parte superior de la capa octaédrica se encuentra otra capa de tetraedros, simétrica a la descrita y con los vértices dirigidos también hacia la capa octaédrica. La capa octaédrica se sitúa, por tanto, entre dos capas tetraédricas, compartiendo con ellas los iones oxo apicales de los tetraedros, y quedando los cationes coordinados a cuatro $\mathrm{O}^{2-}$ y dos $\mathrm{OH}^{-}$. Estas capas constituyen una lámina, por lo que las esmectitas son silicatos TOT.

Las principales diferencias entre la saponita y la montmorillonita se centran en la ocupación de las capas tetraédrica y octaédrica. En la saponita hay una alta sustitución isomórfica en la capa tetraédrica, muy superior a la de la montmorillonita, lo que resulta importante para posibles aplicaciones catalíticas. En cuanto a la capa octaédrica, en la fórmula límite de la saponita no hay sustituciones 
isomórficas ni vacantes catiónicas, por lo que está ocupada por seis cationes $\mathrm{Mg}(\mathrm{II})$, siendo nula su carga negativa. Esta fórmula límite se presenta en muchas saponitas naturales, y en otras el grado de ocupación por cationes $\mathrm{Mg}(\mathrm{II})$ es muy próximo al total, pudiendo llegar a ocurrir que un pequeño grado de sustitución isomórfica de este catión por $\mathrm{Al}(\mathrm{III})$ o $\mathrm{Fe}(\mathrm{III})$ lleve incluso a una ligera carga positiva sobre esta lámina. En el caso de la montmorillonita, esta capa está ocupada, en la idealidad, por 4 cationes $\mathrm{Al}(\mathrm{III})$, pero las muestras reales presentan un grado de ocupación menor, lo que unido a las sustituciones isomórficas por $\mathrm{Mg}(\mathrm{II})$, hace que la carga de esta arcilla esté mayoritariamente situada sobre esta capa. Globalmente, la carga laminar de la saponita es, en general, mayor que la de la montmorillonita.

Por tanto, las principales diferencias entre montmorillonita y saponita pueden resumirse en el origen de la carga (octaédrica-tetraédrica) y en la composición de la capa octaédrica (alumínica-magnésica, respectivamente). En la Figura 1.4 se muestra la estructura y composición de la saponita ideal.

Por otro lado, la saponita también se asemeja a la vermiculita, tanto por la alta carga que ambas presentan como por la composición magnésica de sus capas octaédricas [6].
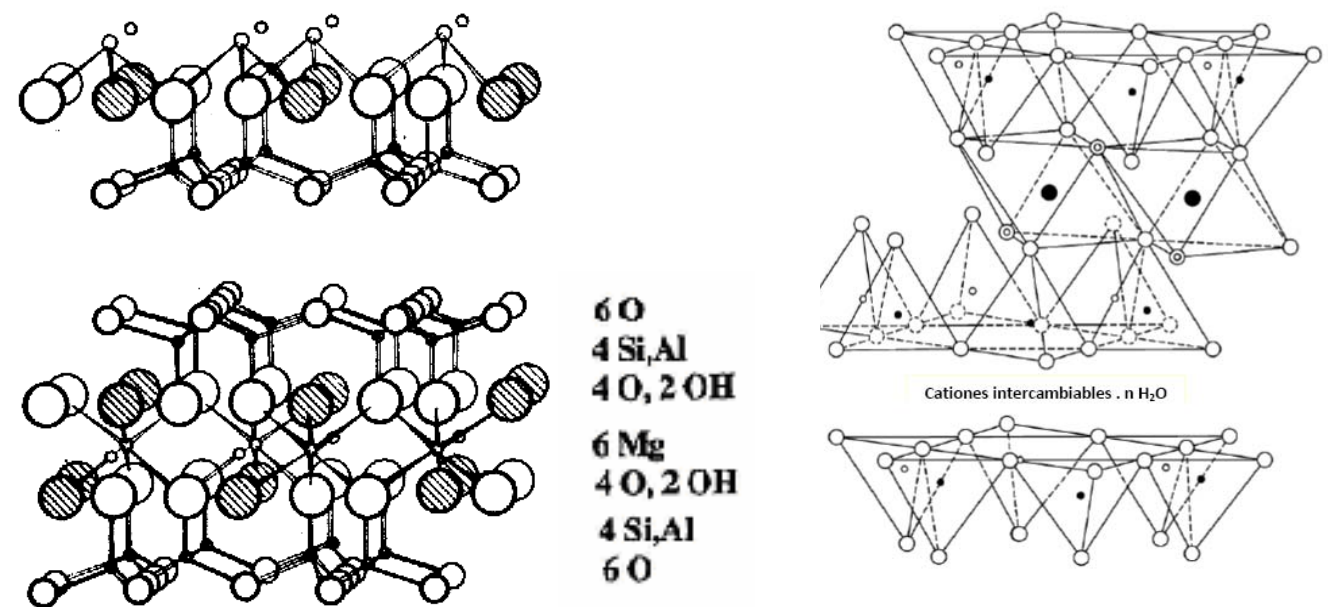

Figura I.4. Estructura de la saponita, adaptada del modelo de Hofmann para la montmorillonita.

Como se ha indicado, las láminas se extienden indefinidamente en dos dimensiones, ejes a y b, apilándose según el eje c. El enlace entre las láminas viene 
dado por los átomos de oxígeno unidos débilmente a los cationes interlaminares. Por ello, las láminas pueden expandirse a lo largo del eje c al penetrar entre ellas moléculas polares, con lo que el espaciado basal de estas arcillas puede fluctuar en un amplio intervalo, presentando un valor mínimo de 9,6 $\AA$ cuando las láminas están lo más próximas posible, y siendo de 12 a $14 \AA$ en las muestras naturales, dependiendo del tamaño de los cationes de cambio y del grado de hidratación. Las láminas pueden expandirse hasta espaciados mucho mayores cuando penetran entre ellas moléculas del tamaño adecuado. Esta expansibilidad viene influida por la naturaleza de los cationes interlaminares, la densidad de carga superficial y la localización de dicha carga.

Los filosilicatos pueden clasificarse de maneras muy diversas, la más sencilla se basa en una diferenciación en función de su estructura, si es laminar o fibrosa. La gran mayoría de los filosilicatos presentan estructura laminar por lo que, a su vez, se dividen en función del tipo de lámina, del número de capas tetraédricas y octaédricas por lámina, del defecto de carga de las láminas, refiriéndolo a la celda unidad, y de la ocupación de la capa octaédrica, si es dioctaédrica o trioctaédrica. Atendiendo a estos parámetros, los filosilicatos se clasifican como se indica en la Tabla I.1.

\section{I.5. Propiedades físico-químicas de las arcillas}

Como se ha indicado, la diversidad de propiedades de las arcillas se debe fundamentalmente a su estructura y composición, por lo que dichas propiedades pueden ser abordadas desde diferentes enfoques en función de las características de los sólidos finales y las aplicaciones para las que vayan a ser utilizados. En muchas aplicaciones, es fundamental un estudio detallado de las propiedades reológicas, mientras en otros casos prima la evaluación estructural o la cristalinidad. A continuación se revisan las propiedades que aportan mayor información cara a las aplicaciones objeto del presente trabajo. 


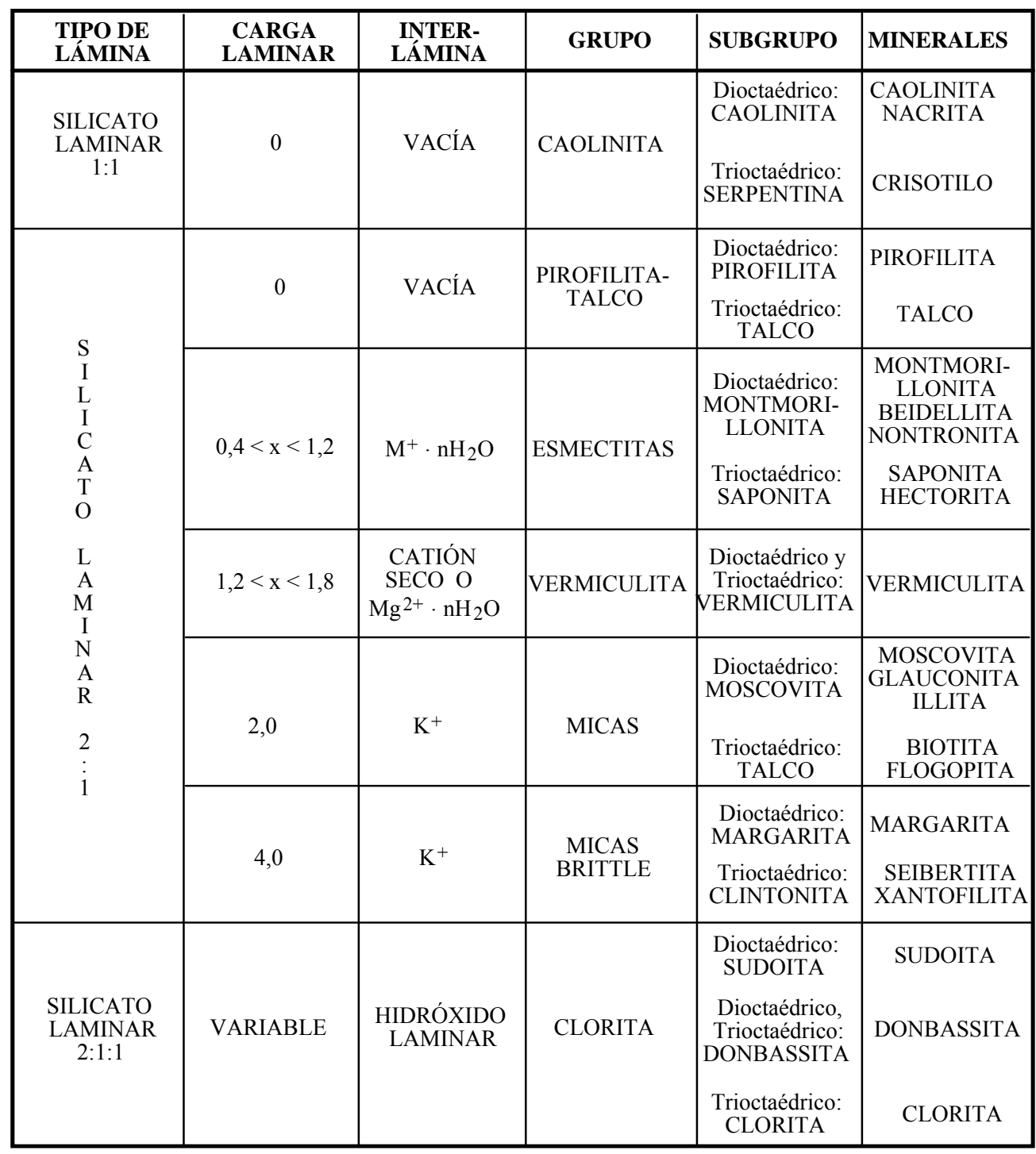

Tabla I.1. Clasificación de los minerales arcillosos laminares. Adaptada de [7].

\section{I.5.1. Capacidad de intercambio catiónico}

Como ya se ha comentado, las sustituciones isomórficas que tienen lugar en las capas tetraédricas y octaédricas de las arcillas dan lugar a cargas negativas netas en las láminas, que son compensadas por cationes que se sitúan en el espacio interlaminar. La cantidad de carga positiva que es aportada por estos cationes para neutralizar la carga de las láminas recibe el nombre de capacidad de intercambio catiónico, ClC (también se utilizan la denominación capacidad de cambio catiónico, abreviada CCC, así como las siglas en inglés, CEC). La determinación de la CIC es una medida de la carga laminar del mineral $[3,8]$. 
La $\mathrm{ClC}$ de un mineral se puede estimar mediante el grado de reversibilidad de la adsorción de los cationes interlaminares, en función de la fortaleza de la interacción de los cationes con las láminas de la arcilla, mediante las propiedades de hidratación y mediante la localización y número de grupos $\mathrm{OH}^{-}$estructurales. Estas propiedades conducen a valores de $\mathrm{ClC}$ diferentes. En el caso de los minerales arcillosos, se define la $\mathrm{CIC}$ como la cantidad de cationes intercambiables a un pH dado [9]. La unidad recomendada por la IUPAC para esta magnitud es $\mathrm{cmol}(+) / \mathrm{kg}$, que es numéricamente equivalente a meq/100g arcilla, unidad tradicionalmente utilizada en este campo de investigación. En el caso particular de las esmectitas, se encuentran valores de CIC entre 70 y 120 meq/100 g de arcilla.

Aunque la $\mathrm{CIC}$ de un mineral arcilloso se relacione con la carga laminar, los valores determinados experimentalmente indicarán únicamente los cationes que son realmente intercambiables en las condiciones de medida. De esta forma, la capacidad de intercambio catiónico corresponderá a la suma de dos tipos de cargas:

a. Sustituciones isomórficas y vacantes en las láminas de la arcilla: Esta carga tiene su origen en la propia estructura de las arcillas y se genera por las sustituciones o vacantes en las capas tetraédricas y octaédricas. En la mayoría de los casos esta deficiencia de carga da lugar a cationes intercambiables, sólo en algunos casos existen interacciones específicas de las capas tetraédricas que pueden impedir el intercambio catiónico en la interlámina. Esto ocurre en las illitas y micas, en las que el potasio situado en el espacio interlaminar no se intercambia ya que se encuentra fuertemente fijado en las cavidades di-trigonales de las capas tetraédricas. La carga generada por sustitución isomórfica se denomina carga constante o permanente [10].

b. Coordinación catiónica en los bordes de las láminas: En función del pH de la disolución en la que se encuentre una arcilla los cationes de los bordes de las láminas se encuentran coordinados con $\mathrm{H}_{3} \mathrm{O}^{+}, \mathrm{H}_{2} \mathrm{O} \circ \mathrm{OH}^{-}$. Esta carga, además de con el pH, puede variar con la fuerza iónica de la disolución. Al depender de estas propiedades, esta carga se denomina variable y se suele determinar a pH neutro. La contribución de esta carga 
depende fundamentalmente de la morfología de las partículas y de la relación entre el área superficial de los bordes y de las caras. En el caso de las esmectitas, suele suponer entre el 10 y el $20 \%$ del total de la carga del mineral arcilloso [11].

Existen en la bibliografía muchos métodos para determinar la capacidad de intercambio catiónico, como el intercambio con protones, el intercambio con especies orgánicas (azul de metileno), el intercambio con $\mathrm{NH}_{4}{ }^{+} \mathrm{o}$ el uso de cationes alcalinos y alcalinotérreos. Debido a las numerosas variables que pueden influir en el proceso y que los resultados obtenidos pueden variar considerablemente de un método a otro, es conveniente utilizar un protocolo estandarizado, de forma que se puedan obtener resultados reproducibles y comparativos entre diferentes muestras.

\section{I.5.2. Propiedades estructurales}

La estructura laminar y la elevada cristalinidad son dos de las propiedades estructurales más interesantes que presentan las arcillas. Debido a la gran diversidad de estructuras que se pueden encontrar en las arcillas resulta difícil su identificación y cuantificación.

La difracción de Rayos $X$ en polvo (DRX-P) es la técnica más utilizada para la identificación de estos minerales, aunque puede llevarse a cabo más fácilmente a partir de patrones de difracción de partículas orientadas, ya que éstos presentan un incremento de la intensidad de las reflexiones basales 00l. Los picos hkl no presentan especial utilidad en la identificación ya que todos los minerales arcillosos son muy similares en cuanto a sus direcciones $x$ e y [12].

El ángulo en el que aparecen las señales en un difractograma es la información más útil que se puede extraer para la identificación de las especies presentes. Otra característica importante de las señales de las arcillas es que presentan anchos de pico mayores que las especies bien cristalizadas, como el cuarzo, debido a que a medida que decrece el tamaño de partícula de un dominio cristalino, la señal difractométrica asociada se vuelve notablemente más ancha.

Por otra parte, la reflexión 060, producida dentro de la lámina e independiente del apilamiento de láminas, es muy sensible a la naturaleza de los cationes 
octaédricos, presentando valores cercanos a 1,48 $\AA$ en esmectitas dioctaédricas y próximos a $1,53 \AA$ en el caso de esmectitas trioctaédricas. Esta reflexión está vinculada al parámetro $b$ de la celda y es independiente del eje c por lo que su estudio permite distinguir entre arcillas dioctaédricas y trioctaédricas.

\section{I.5.3. Propiedades texturales}

Una de las propiedades de los materiales arcillosos que guarda mayor relación con aplicaciones en catálisis y adsorción es su área superficial específica. Dada la estructura laminar de las arcillas, la superficie específica debida a los bordes es muy inferior a la de sus caras. Aunque el valor teórico de la superficie de estos materiales, teniendo en cuenta su densidad, el tamaño de las partículas y el número de láminas que forman los tactoides, es aproximadamente $800 \mathrm{~m}^{2} / \mathrm{g}$, los valores experimentales generalmente oscilan entre 30 y $80 \mathrm{~m}^{2} / \mathrm{g}$, debido a las diferentes formas de apilamiento de las láminas y al colapso parcial de la estructura laminar.

Entre los tratamientos físicos y químicos que pueden realizarse sobre los materiales arcillosos esmectíticos, la intercalación/pilarización es uno de los que produce un mayor un incremento de la superficie específica; los sólidos pilareados finales suelen presentar valores superiores a los $400 \mathrm{~m}^{2} / \mathrm{g}$. El aumento de la superficie específica se debe principalmente al desarrollo de una estructura microporosa accesible a la adsorción de nitrógeno, y que depende del espaciado interlaminar y de la distancia entre los pilares.

\section{I.5.4. Propiedades catalíticas}

Los minerales arcillosos son excelentes materiales para el desarrollo de catalizadores, al ser catalizadores en sí mismos y poder actuar como soportes de otras fases activas. Además, pueden permitir un aumento de la selectividad gracias a las restricciones de tamaño y de forma impuestas por las dimensiones de los poros [13].

Los primeros trabajos de investigación realizados en este campo con materiales arcillosos se basaron en el tratamiento ácido o intercambio iónico para incrementar 
su acidez Brönsted o Lewis. Así, arcillas tratadas mediante activación ácida han sido ampliamente utilizadas en procesos catalíticos como el craqueo del petróleo. En la actualidad, el uso se he diversificado y los minerales arcillosos son reconocidos como catalizadores eficientes en reacciones que incluyen transformaciones redox, síntesis orgánica, Química Fina, etc. [14].

Las características de las arcillas que condicionan su actividad catalítica han sido recientemente analizadas por Adams y McCabe [13], haciendo hincapié en las siguientes propiedades:

a. Baja dimensionalidad: La bidimensionalidad de los canales porosos presentes en la estructura de las arcillas hace que las moléculas situadas en los mismos tengan más propensión a chocar entre ellas de lo que podrían hacerlo en tres dimensiones, dando lugar a una mayor reactividad.

b. Características estructurales: La presencia de especies de aluminio y/o hierro en los bordes de las láminas proporciona a los sólidos arcillosos acidez tipo Lewis. Además, la disociación de moléculas de agua presentes en la interlámina y coordinadas a cationes de alta carga y bajo radio, como $\mathrm{Al}^{3+}, \mathrm{Fe}^{3+} \circ \mathrm{Cr}^{3+}$, proporciona acidez tipo Brönsted. Ambos tipos de acidez permiten la catálisis de un buen número de reacciones. La actividad redox de las arcillas se puede obtener mediante la intercalación de cationes activos en el espacio interlaminar, como $\mathrm{Cu}^{2+}$ o $\mathrm{Fe}^{3+}$.

c. Selectividad de tamaño y/o forma: Originalmente, las arcillas pilareadas se prepararon para intentar solventar las desventajas que presentaban las arcillas tratadas mediante intercambio iónico o activación ácida frente al uso catalítico de las zeolitas. La pilarización ofrece la ventaja adicional de que los pilares presentan centros activos frente a la catálisis y que, en función del tamaño y la distribución de los pilares, se forman canales moleculares que imponen selectividad de forma y tamaño en las reacciones que catalizan.

d. Catálisis inorgánica: Los minerales arcillosos son históricamente conocidos como catalizadores de reacciones orgánicas, sin embargo, recientemente se ha documentado su uso en catálisis de reacciones 
inorgánicas, como el intercambio de ligandos en complejos sustitucionalmente inertes de $\mathrm{Cr}(\mathrm{III})$ y $\mathrm{Co}(\mathrm{III})$, donde las arcillas crudas parecen actuar como catalizadores básicos intercambiado protones desde la disolución reaccionante.

e. Soportes catalíticos: Dada su alta superficie específica, sobre todo en el caso de sólidos modificados, las arcillas pueden actuar como excelentes soportes de especies reactivas, y también pueden proporcionar varios tipos de centros activos que resultan co-activos con las especies soportadas.

Algunas modificaciones físico-químicas de las arcillas, particularmente la intercalación/pilarización, permiten una mejora de estas propiedades de los sólidos, favoreciendo su utilización catalítica en muchas reacciones de gran importancia industrial. Con ello, el estudio de las aplicaciones catalíticas de las arcillas, tanto naturales como modificadas, se ha incrementado notablemente en las últimas décadas, como puede comprobarse en trabajos de revisión, como los de Adams y McCabe y Gil et al. $[13,14]$.

\section{I.6. Aplicaciones de las arcillas}

Los materiales arcillosos han sido muy empleados desde la antigüedad, principalmente en construcción y usos cerámicos y medicinales. En el último siglo han comenzado a ser utilizados industrialmente y a gran escala. Hoy en día, tienen una gran importancia económica y sus aplicaciones comprenden un gran abanico de posibilidades.

Desde el punto de vista industrial, la mayor parte de las aplicaciones no requieren especificaciones estrictas en cuanto a la composición química (composición de las capas tetraédrica y octaédrica). Sin embargo, en algunos casos sí se presta especial atención a la química del espacio interlaminar y a sus propiedades físico-químicas. Las distintas familias de materiales arcillosos presentan propiedades diferentes que determinan sus posteriores usos; a continuación se citan las familias más utilizadas. 
- Las arcillas comunes encuentran su principal aplicación en el campo de la cerámica y la construcción (tejas, ladrillos, tubos, baldosas...), alfarería tradicional, lozas, etc. A estos usos se han destinado desde el principio de la Humanidad. Además, son utilizadas en la manufactura de cementos, como fuentes de alúmina y sílice, y en la producción de áridos ligeros.

- El caolín es un mineral muy importante desde el punto de vista industrial. Adquirió gran fama en el siglo XVI gracias a la porcelana china, fabricada a base de pastas ricas en este mineral. En la actualidad, su uso principal es la fabricación de papel, en la que se utiliza para proporcionar el acabado superficial o estucado, y que consume más del $50 \%$ de la producción mundial de este mineral. Este proceso requiere unas especificaciones de calidad muy estrictas, tanto en la pureza como en el color o el tamaño de grano. También es importante el uso de este mineral en la fabricación de materiales cerámicos (porcelana, gres, loza sanitaria o de mesa), y refractarios (aislantes térmicos y cementos). Al igual que en el caso del papel, las especificaciones necesarias para estos usos son también muy estrictas, tanto en pureza como en tamaño de grano. Otros usos importantes del caolín son la obtención de fibra de vidrio, catalizadores, absorbentes estomacales, industria cosmética, resinas y pinturas.

- Las bentonitas, nombre industrial de los minerales ricos en esmectitas, tienen un uso industrial muy importante y variado. Se utilizan en gran escala para la fabricación de moldes de fundición, donde el reciclado no suele ser posible ya que, debido a las altas temperaturas alcanzadas, pierden irreversiblemente parte de su agua de constitución, perdiendo así sus propiedades. La elevada superficie de las bentonitas les confiere una gran capacidad tanto de absorción como de adsorción, propiedad que es aprovechada para la clarificación y decoloración de aceites, vinos y sidras, así como en la purificación de aguas contaminadas con aceites industriales o disolventes orgánicos. En los últimos años se ha desarrollado el uso de estas arcillas como material de sellado para residuos tóxicos y peligrosos, como los productos radiactivos de actividad baja y media. En ingeniería civil, las bentonitas se usan para cementar fisuras y grietas de las rocas, absorbiendo la humedad para impedir el derrumbamiento de túneles o excavaciones. Otra aplicación de las bentonitas que está cobrando importancia en los últimos años es su utilización como ligante en la fabricación de alimentos pelletizados para animales e incluso 
para humanos, particularmente en salchichas y similares. Además de su acción como ligante, sirve de soporte de vitaminas, sales minerales, antibióticos y otros aditivos de la alimentación animal. La industria farmacéutica usa estas arcillas como excipientes dado que no son tóxicas ni irritantes, y no son absorbidas por el cuerpo humano. Actúan como adsorbentes, estabilizantes, espesantes, agentes suspensores o modificadores de la viscosidad.

El mineral arcilloso utilizado en este trabajo es la saponita. Aunque ya se han comentado de forma general algunas de sus características, a continuación se discuten de forma más detallada.

\section{I.7. Características generales de la saponita}

La saponita fue descubierta por Cronstedt en 1758 en Inglaterra, e inicialmente llamada "terra lemmia" por pertenecer a una tierra de batán. Este material arcilloso se usaba frecuentemente como sustituto del jabón, uso del que deriva su nombre actual. En 1931, Ross y Kerr la clasificaron en el grupo de las esmectitas.

Históricamente, las muestras de saponita más estudiadas provienen de los yacimientos de Kozákov (República Checa), Grösslattengrün (Alemania), Griffith Park y Ballarat (California, USA), y Milford (Utah, USA). Los yacimientos actualmente más importantes se sitúan en diversos lugares de Estados Unidos, Japón, Australia y Turquía. En España existen importantes yacimientos en la Cuenca del Tajo (provincias de Madrid y Toledo), siendo éste el origen de la saponita utilizada en el presente trabajo.

La fórmula estructural tipo de una saponita puede expresarse como [ $\mathrm{Si}_{8-\mathrm{x}} \mathrm{Al}_{\mathrm{x}}$ ] [ $\left.\mathrm{Mg}_{6}\right] \mathrm{O}_{20}(\mathrm{OH})_{4} \mathrm{M}^{+}{ }_{x}$, donde $\mathrm{M}^{+}$es el conjunto de los cationes de cambio del silicato, dados en forma de catión monovalente. Es, como ya se ha indicado, una esmectita trioctaédrica ya que en su fórmula ideal, su capa octaédrica está ocupada por seis cationes $\mathrm{Mg}^{2+}$, no quedando ninguna posición vacante. En las muestra reales, se producen sustituciones isomórficas de $\mathrm{Mg}^{2+}$ por otros cationes, especialmente por $\mathrm{Al}^{3+}, \mathrm{Fe}^{2+}$ y $\mathrm{Fe}^{3+}$, y cantidades menores de otros metales como $\mathrm{Mn}^{2+}$ y $\mathrm{Ti}^{4+}$. Las sustituciones isomórficas por $\mathrm{Fe}^{2+}$ dan lugar a una serie de disoluciones sólidas que van desde el término completamente magnésico, la saponita propiamente dicha, 
hasta el término ferroso, que suele denominarse saponita ferrosa, y que responden

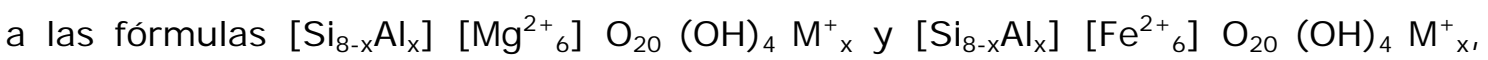
respectivamente.

La saponita tiene una capacidad de cambio de cationes elevada, con valores que varían entre 90 y $100 \mathrm{meq} / 100 \mathrm{~g}$, variación que depende fundamentalmente del origen de las muestras. Su densidad es del orden de $2,5 \mathrm{~g} / \mathrm{cm}^{3}$ y presenta colores que van desde el amarillo pálido hasta el marrón oscuro dependiendo fundamentalmente de su contenido en hierro.

Las saponitas naturales generalmente presentan valores de superficie específica entre $30-80 \mathrm{~m}^{2} / \mathrm{g}$, como es habitual para el grupo de las esmectitas. No obstante, las saponitas de la Cuenca del Tajo presentan valores considerablemente mayores, hasta $300 \mathrm{~m}^{2} / \mathrm{g}$, debido a su origen sedimentario y su pequeño tamaño de partícula.

La saponita es una arcilla idónea para su modificación por diversos tratamientos. En el caso de la activación ácida, su composición mayoritariamente magnésica hace que la disolución parcial de la capa octaédrica pueda hacerse con disoluciones muy diluidas. En el caso de la intercalación/pilarización, su alta carga y su origen tetraédrico favorecen enormemente este tratamiento, y además proporcionan a los sólidos finales una acidez mayor y más fuerte que la obtenida por intercalación/pilarización de montmorillonita.

Todas estas características han hecho que en los últimos años se haya incrementado notablemente la investigación sobre la saponita, aunque la montmorillonita siga siendo la esmectita más estudiada, principalmente por su mayor disponibilidad en la naturaleza.

\section{I.8. Intercalación y pilarización}

Las arcillas pilareadas, también denominadas arcillas intercaladas, arcillas apilaradas, arcillas pilarizadas, arcillas ancladas o PILC's, todos ellos términos derivados del inglés "Pillared clays", constituyen una de las familias de sólidos microporosos desarrollados por ingeniería molecular más estudiadas en los últimos años. El proceso de intercalación fue descrito por Barrer a mediados de los 50 del siglo 
pasado, tras utilizar cationes orgánicos, amonio y quelatos metálicos, para obtener sólidos que, aunque resultaron térmicamente inestables, fueron el primer paso para el desarrollo de las arcillas pilareadas con cationes inorgánicos preparadas a finales de los años 70. Las primeras esmectitas intercaladas con polioxocationes de aluminio con

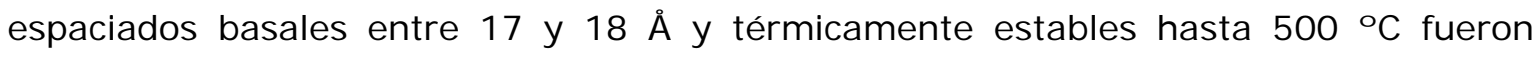
descritas de forma independiente por Lavah et al., Brindley y Sempels, y Vaughan y Lussier $[15,16]$.

La primera etapa de la preparación de estos materiales consiste en el intercambio de los cationes interlaminares de una esmectita por policationes inorgánicos voluminosos. Los sólidos resultantes son calcinados posteriormente a temperaturas relativamente altas para convertir los policationes en especies térmicamente estables. La primera etapa hace que aumente considerablemente el espaciado interlaminar de la arcilla, por lo que los sólidos intercalados, habitualmente llamados "arcillas intercaladas" y que pueden ser considerados como precursores de las auténticas arcillas pilareadas, tienen un elevado espaciado basal. Durante la posterior calcinación, los policationes son convertidos en fases próximas a óxidos metálicos, denominadas "pilares", que quedan insertados entre las láminas de la arcilla manteniéndolas separadas entre sí, evitando de esta manera el colapso de la estructura. El proceso debe dar lugar a un aumento del espaciado basal $\mathrm{d}_{001}$, observable por difracción de rayos $X, y$ a un incremento de la superficie específica, que deben producirse simultáneamente para que el sólido final pueda verdaderamente denominarse arcilla pilareada [15].

Dada la gran variedad de arcillas y de especies pilareantes que se pueden utilizar, la aplicación final que se vaya a dar al sólido pilareado puede estar influida por varios factores que afectan al proceso de preparación. Entre esos factores destacan la estabilidad térmica o hidrotérmica de las arcillas pilareadas, su distancia interlaminar, la densidad de los pilares incorporados, la naturaleza química de dichos pilares y su estabilidad química.

El policatión más utilizado en experimentos de intercalación de arcillas es, sin lugar a dudas, el $\left[\mathrm{Al}_{13} \mathrm{O}_{4}(\mathrm{OH})_{24}\left(\mathrm{H}_{2} \mathrm{O}\right)_{12}\right]^{7+}$, habitualmente llamado policatión de Keggin de $\mathrm{Al}$, o simplemente $\mathrm{Al}_{13}$. Su estructura se representa en la Figura I.5. Este policatión es mucho más conocido que los formados por otros elementos, se prepara fácilmente y 
es bastante estable, por lo que las arcillas pilareadas con Al, habitualmente denominadas Al-PILCs, son las mejor conocidas. Otros cationes que polimerizan dando especies cargadas positivamente que pueden ser usadas en experimentos de intercalación son, principalmente, $\mathrm{Zr}^{4+}, \mathrm{Ti}^{4+}, \mathrm{Cr}^{3+}, \mathrm{Fe}^{3+}$ y $\mathrm{Ga}^{3+}$. En el caso del $\mathrm{Cr}^{3+}$, usado en este trabajo, puede polimerizar formando el dímero $\left[\mathrm{Cr}_{2}(\mathrm{OH})_{2}\left(\mathrm{H}_{2} \mathrm{O}\right)_{8}\right]^{4+}$, el trímero $\left[\mathrm{Cr}_{3}(\mathrm{OH})_{4}\left(\mathrm{H}_{2} \mathrm{O}\right)_{9}\right]^{5+}$, dos tetrámeros: uno abierto $\left[\mathrm{Cr}_{4}(\mathrm{OH})_{6}\left(\mathrm{H}_{2} \mathrm{O}\right)_{11}\right]^{6+}$ y el otro cerrado $\left[\mathrm{Cr}_{4}(\mathrm{OH})_{5}\left(\mathrm{H}_{2} \mathrm{O}\right)_{10}\right]^{5+}$, y especies hexámeras. La estructura de estos oligómeros también se presenta en la Figura I.5.

Por otra parte, puede realizarse la intercalación conjunta con dos o más elementos, siempre que los mismos polimericen en condiciones similares, o que la polimerización del que se ponga en pequeñas cantidades, que podemos denominar "dopante", no altere significativamente la del elemento mayoritario.
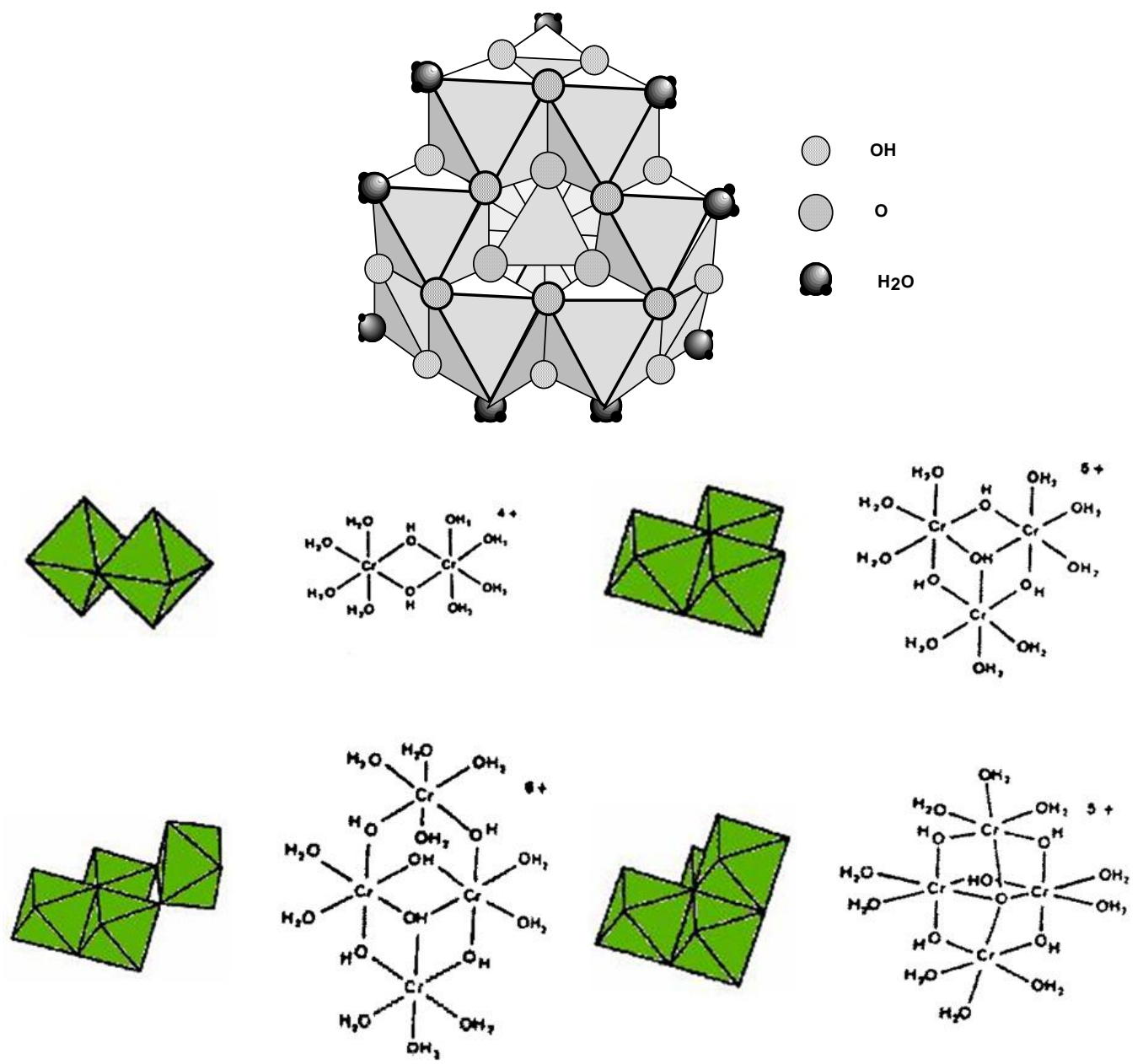

Figura I.5. Estructura del policatión $\mathrm{Al}_{13}$-Keggin y de los oligómeros formados por el $\mathrm{Cr}^{3+}$. 
Además de la naturaleza del policatión, otros factores importantes que condicionan la intercalación son el tiempo y las condiciones de hidrólisis y envejecimiento, y la concentración del policatión. El procedimiento experimental general para la obtención de una arcilla pilareada con policationes de Al puede resumirse mediante el esquema mostrado en la Figura I.6.

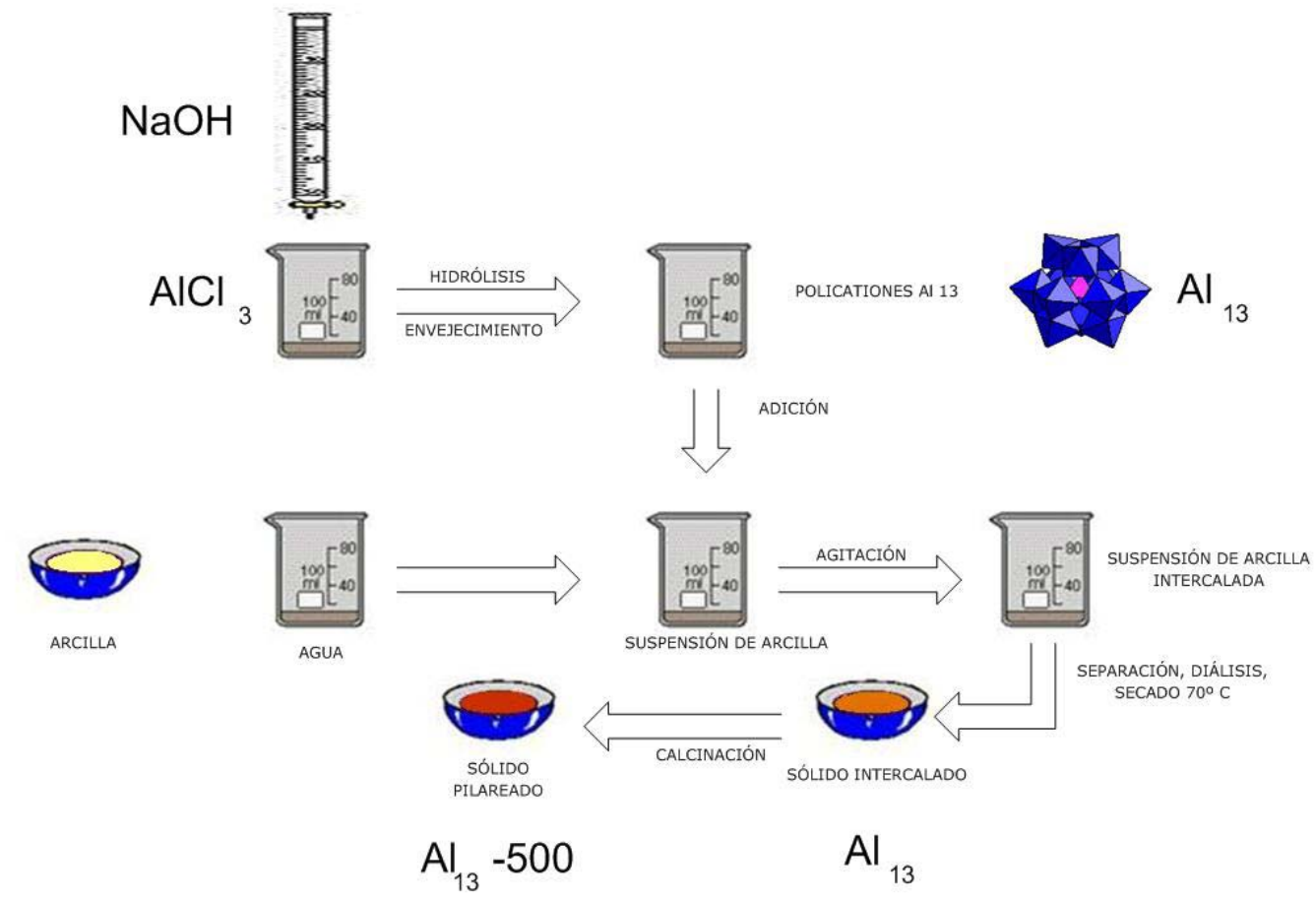

Figura I.6. Representación esquemática del procedimiento experimental para la pilarización de una esmectita con policationes de Al.

En la Figura 1.7 se ofrece el esquema de la estructura de las arcillas pilareadas, así como los cambios experimentados durante el proceso de preparación de estos sólidos, con especial atención a los cambios de las especies presentes en su región interlaminar. 

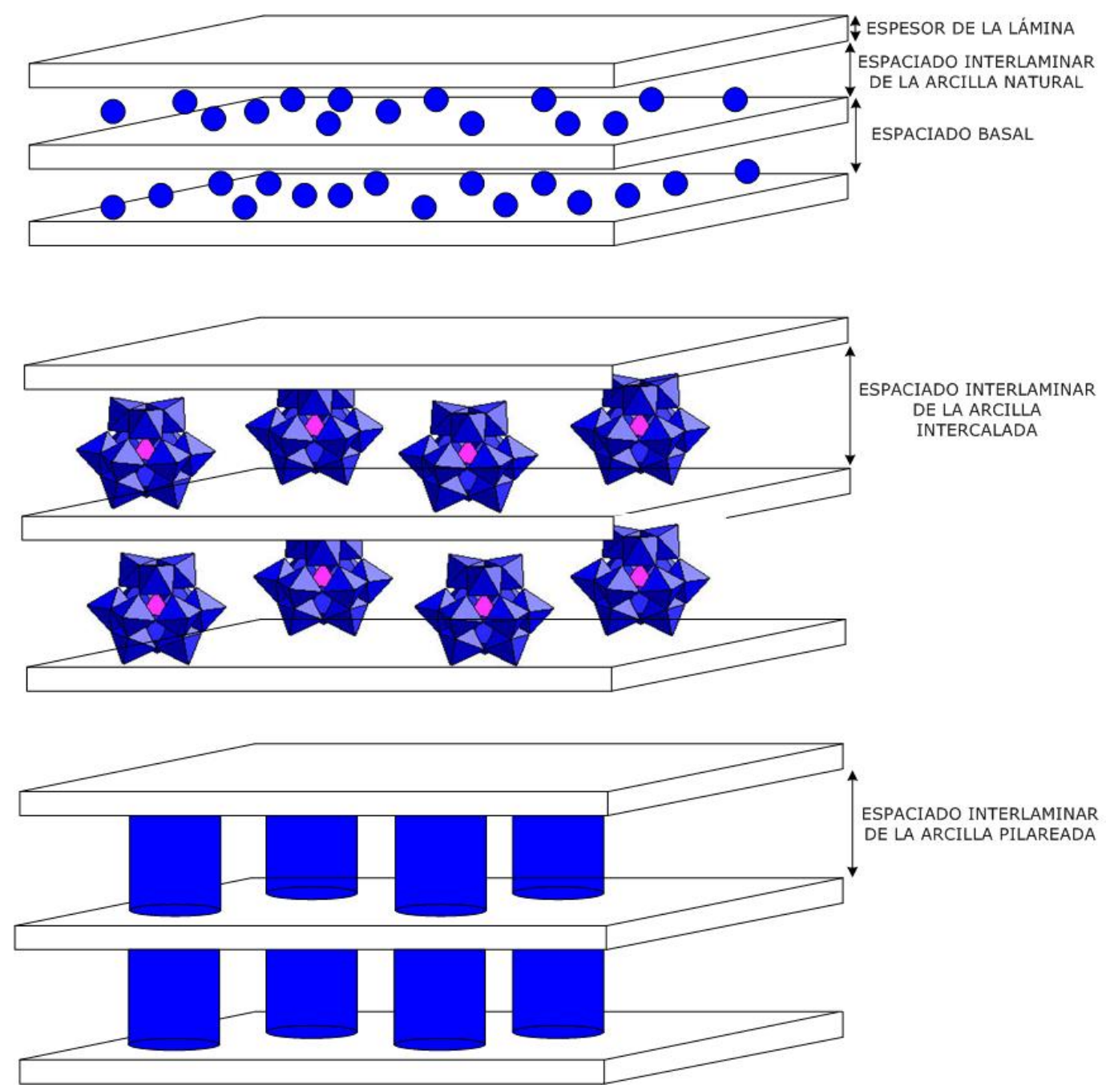

Figura I.7. Estructura de una arcilla pilareada con definición gráfica de la evolución de algunos parámetros durante su preparación.

\section{I.9. Procesos catalíticos}

La capacidad de una sustancia de actuar como catalizador de una reacción concreta depende fundamentalmente de su naturaleza química. En el caso de la catálisis heterogénea, la capacidad catalítica del sólido considerado dependerá de las propiedades químicas de su superficie, siendo éstas un reflejo de la química del sólido.

Para que ocurra un fenómeno catalítico es necesaria la interacción química entre el catalizador y el sistema reactivos-productos. Esta interacción no debe modificar la naturaleza química del catalizador, a excepción de su superficie, lo que implica 
que la interacción entre el catalizador y el sistema reaccionante se produzca únicamente en la superficie del catalizador y no involucre el interior del sólido.

Los fenómenos catalíticos heterogéneos requieren la adsorción química en la superficie del catalizador de al menos uno de los reactivos. Dado que la reacción se lleva a cabo en la superficie del catalizador, el conocimiento de la cantidad de moléculas adsorbidas en esta superficie reviste gran importancia. El sistema catalítico heterogéneo está constituido por un fluido que es una reserva de moléculas por transformar o ya transformadas y una superficie (catalizador), por lo que la cantidad de reactivo adsorbido se relaciona con su concentración en la fase gas.

\section{I.9.1. Catalizadores soportados}

La utilización de catalizadores soportados en lugar de catalizadores másicos responde a la necesidad de maximizar la eficacia disminuyendo los costes, puesto que, al ser la catálisis un fenómeno de superficie, permite alcanzar el mismo efecto catalítico con una menor cantidad de fase activa. Las principales características que se buscan en un catalizador son la actividad y la durabilidad, y también son importantes su idoneidad y su alta superficie específica. Además, hay que tener en cuenta que los catalizadores han de poder trabajar a altas temperaturas y presiones, o bien ser capaces de reducir éstas y permitir que la reacción transcurra en condiciones semejantes a las ambientales. Algunas de las principales ventajas que conlleva la dispersión de una fase activa sobre un soporte son la disminución del coste, ya que se necesita menor cantidad de fase activa, una mayor actividad, debido a que tendremos una mayor superficie accesible, y la selectividad y regenerabilidad que se obtienen mediante la dispersión de una fase activa sobre un soporte sólido. Además, el soporte facilita la manejabilidad del catalizador, especialmente en el caso de fases activas tóxicas, peligrosas o con baja biodegradabilidad [17].

La resistencia mecánica del catalizador soportado es muy importante para minimizar su desgaste, debido al contacto con los reactivos, y así incrementar su durabilidad. Además de la fricción física con los reactivos y el reactor, que tiene carácter irreversible y una repercusión importante en el área superficial, la otra 
causa más común del deterioro es el envenenamiento del catalizador debido a impurezas en los reactivos o a reacciones secundarias no deseadas.

Los principales componentes de un catalizador soportado son los siguientes:

- Fase activa: es el catalizador propiamente dicho. También se conoce como principio activo del catalizador, o bien centros activos del catalizador, ambos conceptos referidos a la parte de su estructura que participa en la conversión de los reactivos en productos. La fase activa está dispersa sobre la superficie del soporte.

- Promotores: Son aditivos, sin capacidad de catálisis, que afectan al catalizador. Permiten que el catalizador trabaje al $100 \%$ de sus posibilidades. Se distinguen tres tipos de promotores según su funcionalidad:

- Promotor textural: Inhibe la aglomeración de partículas de catalizador, de esta manera se evita la disminución del número de centros activos y del área superficial del catalizador.

- Promotor estructural o electrónico: Exalta el carácter ácido o básico del catalizador, mejorando la capacidad catalítica de éste en reacciones catalizadas por mecanismos ácido-base.

- Promotor anti-envenenamiento: Protege al catalizador inhibiendo aquellas sustancias no deseadas y con efectos tóxicos sobre éste.

- Soporte: Maximiza el área superficial del catalizador disperso sobre él y lo protege de ser arrastrado por los reactivos, proporcionándole resistencia mecánica y durabilidad. Algunos de los soportes más utilizados, como sílice, alúmina, titania, zeolitas, arcillas o carbones, poseen propiedades catalíticas por sí mismos y contribuyen sinérgicamente a la catálisis.

Los soportes que han sido más utilizados son la alúmina, la sílice y las zeolitas, y en segundo lugar los aluminosilicatos y el carbón activo; aunque la literatura describe en los últimos años otros soportes-catalizadores a partir de óxidos mixtos. Aunque estos óxidos pueden encontrarse en minerales y rocas de la naturaleza, es frecuente su síntesis en el laboratorio para controlar y mejorar sus propiedades. 
Para la preparación de catalizadores soportados se necesitan, en principio, todos los componentes antes citados, aunque en muchos casos el soporte contiene centros activos que hacen las funciones de promotores. Es habitual que el catalizador no sea añadido como tal, sino como un precursor que contiene el principio activo y, en una segunda etapa, se fije al soporte. En el proceso de preparación se añaden pequeñas cantidades de estos precursores al soporte, al objeto de que en los sólidos finales estén lo más dispersos posible para incrementar la eficacia del catalizador. El soporte deberá tener el menor tamaño de partícula posible ya que esto implica un aumento en el número de centros activos y en la superficie específica.

Existen numerosos procesos de preparación de catalizadores soportados, las etapas de cada uno de ellos suelen adecuarse al soporte y al catalizador considerados. Normalmente, esta preparación implica tres etapas: Incorporación del precursor al soporte (generalmente en medio acuoso), eliminación del medio acuoso mediante secado, y una posterior calcinación y/o reducción. El gran desarrollo de los catalizadores soportados en los últimos años ha venido acompañado de un perfeccionamiento en las técnicas de impregnación de sólidos y deposición de partículas sobre ellos. Entre las técnicas más generalizadas podemos destacar:

a) I mpregnación simple o en vía húmeda: Es el método más sencillo. Sobre una suspensión del soporte se disuelve la cantidad necesaria de precursor de manera que tanto las partículas de disolvente como las de precursor ocupan todos los poros del soporte. El disolvente se elimina mediante evaporación y secado del sólido, de manera que finalmente las únicas partículas depositadas sobre la superficie y los poros del soporte sean las del precursor.

b) I mpregnación por humedad incipiente: Consiste en mojar el soporte con una cantidad de disolución suficiente para que quede totalmente mojado pero sin disolución sobrante. Controlando pocas variables se consigue un cierto control del modo de deposición del catalizador sobre el soporte. Se utiliza generalmente cuando quieren conseguirse altos rendimientos en la deposición. 
La cantidad de disolución empleada es la mínima posible para disolver el precursor y empapar el soporte, de tal manera que todo el catalizador quede sobre el soporte tras el secado, pudiéndose realizar varias impregnaciones sucesivas si el precursor es poco soluble o si se quiere aumentar la cantidad de fase activa sobre la superficie.

c) Método de intercambio iónico: Este método puede utilizarse para incorporar tanto especies catiónicas como aniónicas, el único requisito es que el soporte contenga grupos hidroxilos (sílice, alúmina, titania y análogos), ya que participan en reacciones de intercambio iónico en medios acuosos. Utilizando un soporte con carácter electropositivo, el óxido formado correspondiente es básico y produce grupos hidroxilo, mientras que si tiene carácter electropositivo, da lugar a un óxido ácido y libera protones. Los protones pueden ser intercambiados por cationes metálicos mientras que los grupos hidroxilos se sustituyen por ligandos cargados negativamente. La calcinación y/o reducción de estos catalizadores da lugar a altas concentraciones de metal con un elevado grado de dispersión sobre la superficie.

d) Método de adsorción-desorción: Es un método válido para soportes fuertemente básicos, donde pueden adsorberse soluciones acuosas metálicas.

e) Coprecipitación: Es otro de los métodos más usados. Sobre una disolución que contiene un precursor del soporte, se añade una disolución básica con el metal catalizador (generalmente de su hidróxido), produciéndose la coprecipitación de ambos elementos y originando óxidos mixtos con elevadas propiedades catalíticas. Alternativamente, puede hidrolizarse de forma controlada una disolución que contenga una sal del elemento componente del soporte, habitualmente $\mathrm{Al}^{3+}, \mathrm{y}$ del componente de la fase activa, precipitando ambos conjuntamente. El aumento controlado del $\mathrm{pH}$ producido por la descomposición térmica de la urea es probablemente el procedimiento más utilizado.

f) Deposición química de vapor: Este método presenta la particularidad de carecer de un medio líquido. El soporte se fija en un horno tubular, al que llega el precursor en forma de vapor. El vapor se adhiere a la superficie del soporte, pudiendo ser necesaria una reducción para obtener el catalizador final. La 
forma de producir el vapor o la temperatura del horno son variables importantes en la preparación de estos catalizadores.

\section{I.10. El método sol-gel y el método de co-precipitación}

Aunque la mayoría de los estudios descritos en la presente Tesis Doctoral se han realizado sobre arcillas naturales, una parte de ellos se ha realizado sobre materiales preparados por el método sol-gel y por co-precipitación, por lo que los mismos serán descritos de manera muy general.

El proceso sol-gel para la preparación de sólidos se conoce desde hace más de 150 años, pero ha sido en las últimas décadas cuando ha experimentado un enorme auge, siendo utilizado para la preparación de vidrios altamente homogéneos, cerámicas, materiales compuestos, ópticos, y catalizadores, entre otros [18].

En general, esta metodología conlleva la preparación de una suspensión de un sólido en un líquido, seguida de la eliminación de este líquido, y finalmente de la densificación del sólido. Este proceso puede ser realizado mediante dos rutas, la hidrolítica o convencional y la no-hidrolítica. La ruta hidrolítica es la utilizada normalmente, y conlleva la hidrólisis de un precursor alcóxido tetrafuncionalizado, usando un catalizador ácido o básico. El proceso no es sencillo y existe la posibilidad de formación de varias especies intermedias, por verse envueltos varios reactivos con distintas reactividades [19].

La ruta no hidrolítica (NHG), fue desarrollada durante la década de los 90 como una alternativa a la hidrolítica. El método se basa en la condensación de un haluro metálico con éter isopropílico, a través de la unión del enlace $O-R$ y formación de un haluro de alquilo, donde el éter actúa como generador de oxígeno. El alcóxido es producido in situ por reacción del haluro con el éter, a través de la formación de un aducto de Lewis, siendo precisamente la generación in-situ del alcóxido la gran ventaja de esta ruta. Este procedimiento permite incorporar sales metálicas, complejos, colorantes orgánicos, porfirinas, etc., antes de la gelificación del material, para su posterior utilización como sensores o catalizadores, entre otros usos [20]. 
Por tanto, la metodología sol-gel permite una combinación, a escala de nanopartículas, de componentes orgánicos e inorgánicos, ofreciendo la posibilidad de sintetizar nuevos compuestos y permitiendo grandes avances en la ciencia de materiales. Ambas rutas utilizan temperaturas bajas, del orden de 60-100 ㅇ , lo que presenta la ventaja de llevar a la formación de materiales más homogéneos, además de permitir una mayor reproducibilidad en la obtención de los materiales.

En el presente trabajo, se intentó preparar saponita sintética mediante este método, utilizando precursores de $\mathrm{Si}^{4+}, \mathrm{Al}^{3+}, \mathrm{Na}^{+}$y $\mathrm{Ni}^{2+}$. $\mathrm{El} \mathrm{Ni}^{2+}$ fue elegido por su facilidad para situarse en las capas octaédricas de materiales arcillosos y por sus propiedades catalíticas. Alternativamente, se consideró el método de coprecipitación, ya citado en el apartado anterior, consistente en la precipitación, al mismo tiempo, de dos o más elementos. Puede ser realizado a $\mathrm{pH}$ constante o variable. El método a pH variable consiste en la adición de una disolución que contenga sales de los cationes sobre una disolución que contenga hidróxido, o viceversa, habitualmente a temperatura relativamente baja para evitar la precipitación de hidróxidos simples. La preparación a pH constante es similar, pero mantenido el pH constante mediante la adición controlada de una disolución alcalina.

La síntesis, tal como había sido planteada, resultó un fracaso en ambos casos, ya que no se pudo obtener la fase saponita, pero en cambio se obtuvo un interesante nanocompuesto takovita/aluminosilicato, cuya caracterización y actividad catalítica forman parte de esta Tesis, y que ha continuado siendo estudiado con posterioridad por nuestro Grupo.

\section{I.11. Objetivos de este trabajo}

Los minerales arcillosos presentan unas excelentes propiedades físico-químicas, que permiten su utilización en una gran variedad de aplicaciones industriales. No obstante, en muchos casos, particularmente en aplicaciones tecnológicas avanzadas, es necesario realizar ciertas mejoras en las propiedades de los materiales naturales para que dichas aplicaciones sean viables. Muchas de estas mejoras pueden conseguirse mediante tratamientos químicos. 
La intercalación y pilarización de arcillas esmectíticas, principalmente montmorillonitas y saponitas, con policationes derivados de diversos cationes es una línea de investigación en la que nuestro grupo trabaja desde hace unos quince años. Mejorando las propiedades físico-químicas y superficiales de materiales arcillosos se pretende responder a la creciente demanda de nuevos adsorbentes y catalizadores caracterizados por su especificidad, eficacia y bajo coste. En la mayoría de las ocasiones, estos estudios han sido realizados sobre arcillas nacionales (en ocasiones se han utilizado algunas arcillas extranjeras a efectos comparativos), lo cual tiene un valor añadido al utilizar los recursos de nuestro país.

Así, el objetivo principal del trabajo presentado en esta Memoria consiste en la preparación de nuevos catalizadores basados en arcillas y que contengan como especies activas fases de $\mathrm{Cr}$ o $\mathrm{Ni}$. En ambos casos se utilizará como material de partida la saponita del yacimiento de Yunclillos (Toledo). En el caso del $\mathrm{Ni}$, se utilizará también un aluminosilicato sintético de este elemento. Los sólidos obtenidos serán utilizados en reacciones de oxidación.

En el caso del $\mathrm{Cr}$, es posible realizar la intercalación-pilarización de este elemento, ya que el catión $\mathrm{Cr}^{3+}$ puede formar varios oligómeros de pequeño tamaño. Sin embargo, la estabilidad térmica de dichos policationes es baja, por lo que su utilización para la intercalación de arcillas suele ser acompañada del policatión $\mathrm{Al}_{13}$, es decir, realizando la intercalación conjunta de policationes de ambos elementos, que será la metodología utilizada en el presente trabajo, empleando distintas relaciones $\mathrm{Al} / \mathrm{Cr}$ y analizando su efecto en las propiedades de los sólidos finales.

Tanto para el caso del $\mathrm{Cr}$ como para el del $\mathrm{Ni}$, se han preparado diversos catalizadores por impregnación. Se ha utilizado como soporte la saponita pilareada con $\mathrm{Al}_{13}$, incorporando los precursores de estos elementos a partir de diversas sales de los mismos, en el caso del $\mathrm{Cr}$ considerando distintos estados de oxidación, al objeto de analizar la importancia de estos precursores en las propiedades de los sólidos finales. 
En el caso del $\mathrm{Ni}$, considerando que no se ha descrito la polimerización del catión $\mathrm{Ni}^{2+}$, y dada su afinidad por las posiciones octaédricas de los materiales arcillosos, se intentó la preparación en el laboratorio de una saponita de $\mathrm{Ni}$, es decir, de una saponita que contenga únicamente $\mathrm{Ni}^{2+}$ en sus posiciones octaédricas, utilizando para ello una metodología sol-gel. No sé consiguió obtener dicha saponita, pero se obtuvo un nanocompuesto formado por la hidrotalcita de $\mathrm{Ni}$-Al, takovita, y un aluminosilicato amorfo. Dicho nanocompuesto presentó excelentes propiedades, por lo que fue caracterizado y utilizado como catalizador.

Los diversos materiales obtenidos fueron caracterizados por análisis químicos, difracción de rayos $\mathrm{X}$, espectroscopias FT-IR y UV-Vis, análisis térmicos, adsorcióndesorción de nitrógeno y microscopia electrónica. Con esto, se consiguió una caracterización completa de todos los materiales, que permite discutir las diferencias producidas por las distintas formas de incorporación de los metales activos, y su influencia en su posterior comportamiento catalítico.

El comportamiento catalítico de estos materiales fue evaluado en dos tipos de reacciones. La primera de ellas, estudiada tanto con los sólidos que contienen $\mathrm{Cr}$ como con los que contienen $\mathrm{Ni}$, fue la reducción de óxidos de nitrógeno en presencia de un hidrocarburo (propeno). La reducción de compuestos de nitrógeno fue elegida por su gran importancia medioambiental. Los sólidos que contienen $\mathrm{Ni}$ fueron además utilizados en dos reacciones de oxidación de hidrocarburos, la epoxidación de (Z)-cicloocteno y la oxidación de ciclohexano, hasta formar el correspondiente epóxido en la primera de ellas (óxido de cicloocteno) y la denominada mezcla K-A en la segunda (ciclohexanona + ciclohexanol). Los productos obtenidos tienen, en ambos casos, alto valor añadido, por lo que son reacciones utilizadas industrialmente, con el inconveniente de utilizar habitualmente oxidantes muy contaminantes, lo que se evitará en el presente trabajo utilizando como oxidante agua oxigenada.

Esta investigación ha sido realizada con el soporte económico del Gobierno de España, a través de los sucesivos Ministerios encargados de la Investigación Científica, así como del Ministerio de Educación, y de la Junta de Castilla y León, a través de la Consejería de Educación. Para realizar este trabajo se ha contado con la colaboración del Departamento de Química Aplicada de la Universidad Pública de Navarra, del Instituto de Catálisis y Petroleoquímica (CSIC), y del Grupo Sol-Gel de 
la Universidade de Franca (Brasil). Asimismo, se ha contado con el apoyo de TOLSA y Feldespatos del Río Pirón, SA, empresas nacionales dedicadas a la explotación de yacimientos de materiales arcillosos.

\section{I.12. Publicaciones que componen esta Tesis Doctoral}

El trabajo realizado en la presente Tesis Doctoral ha sido recogido en diversos artículos científicos, capítulos de libros y comunicaciones a congresos científicos. En la presente Memoria, los resultados experimentales obtenidos se presentan y se discuten en forma de cuatro artículos científicos y un capítulo de libro. Los cuatro artículos han sido publicados en revistas de relevancia internacional en nuestro campo de investigación, mientras que el capítulo de libro se recoge en un volumen derivado de un congreso científico, en el que los capítulos fueron sometidos a un proceso de revisión similar al de los artículos científicos. Estas publicaciones son:

- Estudio de la preparación de catalizadores cromo-saponita pilareada y de su comportamiento catalítico en la oxidación de propeno. G. Mata, R. Trujillano, M.A. Vicente, C. Belver, S.A. Korili y A. Gil. Páginas 33-48 en “Materiales Arcillosos: de la Geología a las Nuevas Aplicaciones", Editado por M. Suárez, M.A. Vicente, V. Rives y M.J. Sánchez. Salamanca, 2006.

- Chromium-saponite clay catalysts: Preparation, characterizacion and catalytic performance in propene oxidation. G. Mata, R. Trujillano, M.A. Vicente, C. Belver, M. Fernández García, S.A. Korili, A. Gil. Applied Catalysis A: General, 327, 1-12, 2007.

- Preparation and characterization of new Ni-aluminosilicate catalysts and their performance in the epoxidation of (Z)-cyclooctene. K.J. Ciuffi, E.J. Nassar, L.A. Rocha, Z.N. da Rocha, S. Nakagaki, G. Mata, R. Trujillano, M.A. Vicente, S.A. Korili, A. Gil. Applied Catalysis A: General, 319, 153-162, 2007.

- Ni-pillared clays as catalysts for the selective catalytic reduction of nitrogen oxides. C. Belver, G. Mata, R. Trujillano, M.A. Vicente. Catalysis Letters, 123, 3240, 2008. 
- (Z)-cyclooctene epoxidation and cyclohexane oxidation on $\mathrm{Ni} /$ alumina-pillared clay catalysts. G. Mata, R. Trujillano, M.A. Vicente, S.A. Korili, A. Gil, C. Belver, K.J. Ciuffi, E.J. Nassar, G.P. Ricci, A. Cestari, S. Nakagaki. Microporous and Mesoporous Materials, 124, 218-226, 2009.

A continuación se ofrece un resumen general de los resultados más importantes alcanzados en estas publicaciones. Aunque se han estudiado dos sistemas catalíticos claramente diferenciados, los basados en $\mathrm{Cr}$ y en $\mathrm{Ni}$, al haber sido ambos utilizados en la reacción de oxidación de hidrocarburos, se hará un resumen conjunto para todas las publicaciones. Posteriormente se expondrá la calidad de las revistas científicas en las que se han publicado estos resultados, al objeto de avalar la calidad de los mismos.

\section{I.12.1. Resumen general de los resultados obtenidos}

La intercalación de saponita con $\mathrm{Al}, \mathrm{Cr}$, o mezclas de ambos elementos se realiza de forma satisfactoria en todas las muestras preparadas. Los difractogramas de las muestras calcinadas indican que los policationes de cromo son poco estables a altas temperaturas. Introduciendo la relación adecuada $\mathrm{Al} / \mathrm{Cr}$ se pueden obtener sólidos que combinan adecuadamente la estabilidad de los pilares de aluminio con las propiedades catalíticas aportadas por el cromo. La incorporación de cromo mediante el procedimiento de intercalación conjunta conduce a muestras con contenidos de $\mathrm{Cr}$ desde el 0 hasta casi el 25\% en masa, de forma que permiten evaluar el contenido de cromo óptimo para la reacción considerada.

En el caso de los sólidos preparados por impregnación con sales de cromo de saponita previamente intercalada con aluminio, la calcinación produce, en algunos casos, la deslaminación de los sólidos, dependiendo de la sal utilizada como precursora para la impregnación. Al igual que en el método anterior, los catalizadores obtenidos presentan elevados valores de espaciado basal y superficie específica, características que los hacen adecuados para su uso como catalizadores de oxidación.

La actividad catalítica de estos sólidos fue estudiada en la reacción de reducción de óxidos de nitrógeno, utilizando propeno como agente reductor, y en diferentes condiciones de reacción (estas condiciones se detallarán en el próximo capítulo). En 
primer lugar, la reacción se llevó a cabo en ausencia de oxígeno, utilizado cantidades estequiométricas de oxidante y de reductor, según la reacción ideal $\mathrm{C}_{3} \mathrm{H}_{6}+9 \mathrm{NO} \rightarrow 3 \mathrm{CO}_{2}+3 \mathrm{H}_{2} \mathrm{O}+9 / 2 \mathrm{~N}_{2}$, analizando además la posible aparición de otros productos de reacción como $\mathrm{CO}$, procedente de una combustión incompleta del hidrocarburo, y diferentes especies de nitrógeno como $\mathrm{NH}_{3}, \mathrm{HCN} \circ \mathrm{HCNO}$, que indicarían un mecanismo de reacción complejo.

Las conversiones de oxidante y reductor son significativamente distintas, alcanzándose conversiones de hasta el 90\% de hidrocarburo mientras que para el óxido de nitrógeno apenas se alcanza el $45 \%$. En las corrientes iniciales introducidas en el reactor no hay ningún otro agente oxidante que pueda participar en la reacción, por lo que el exceso de conversión del hidrocarburo debe producirse utilizando el propio catalizador como oxidante, en un mecanismo del tipo Mars-VanKrevelen. Este mecanismo de reacción implica una oxidación selectiva por catalizadores que contienen óxidos metálicos con estados de oxidación variables, el catalizador pierde parte de su oxígeno constitucional y, para compensar este hecho, el metal varía su estado de oxidación. En el presente caso, tanto en los catalizadores preparados por intercalación como en los preparados por impregnación, el cromo está en forma de $\mathrm{Cr}(\mathrm{III})$ que tras el proceso de catálisis pasaría a $\mathrm{Cr}(\mathrm{II})$.

A continuación se estudió la reacción en presencia de oxígeno. Para ello, se tomó como punto de partida la reacción anterior, y se disminuyó la cantidad de NO a la mitad, sustituyéndolo por $\mathrm{O}_{2}$ hasta alcanzar de nuevo las cantidades estequiométricas de oxidante. Con ello, en estas condiciones la reacción ideal es $\mathrm{C}_{3} \mathrm{H}_{6}+9 / 2 \mathrm{NO}+9 / 4 \mathrm{O}_{2} \rightarrow 3 \mathrm{CO}_{2}+3 \mathrm{H}_{2} \mathrm{O}+9 / 4 \mathrm{~N}_{2}$.

Con ello, se observan conversiones de hidrocarburo por encima del $90 \%$ en todos los sólidos estudiados, es decir, la presencia de dos oxidantes en la mezcla da como resultado una oxidación de hidrocarburo más elevada, especialmente a temperaturas intermedias. La evolución de los óxidos de nitrógeno también presenta importantes diferencias, siendo más efectiva cuando se introduce oxígeno en la alimentación del reactor. Así, cuando el NO es el único oxidante la reacción empieza a ser notable por encima de los $350 \stackrel{\circ}{ } \mathrm{C}$, mientras que con la mezcla de los dos oxidantes esto ocurre a $250 \stackrel{\circ}{ } \mathrm{C}$. 
El contenido en $\mathrm{Cr}$ también influye notablemente. Considerando las

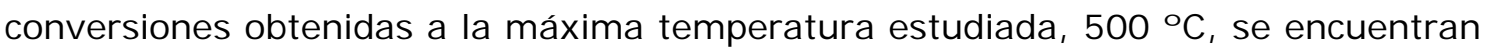
valores hasta un $40 \%$ superiores al utilizar el catalizador con un contenido intermedio de $\mathrm{Cr}$, el preparado con cantidades equimolares de $\mathrm{Cr}$ y Al. No obstante, al aumentar aún más el contenido en $\mathrm{Cr}$, las mejoras de conversión son mucho menos significativas.

Similares resultados se obtuvieron al utilizar los catalizadores preparados por impregnación. En todos los casos, la oxidación del hidrocarburo y la reducción de los óxidos de nitrógeno se ven favorecidas por la mezcla de oxidantes, y en todos los casos las diferencias más notables se observan a temperaturas intermedias.

Si se aumenta la cantidad de oxígeno en la alimentación, se observa que la conversión de propeno es muy elevada aunque la cantidad de oxígeno introducida sea baja, y se mantiene constante independientemente de la cantidad de oxidante introducida en el reactor. Sin embargo, la conversión de NO disminuye de forma importante a medida que se introduce oxígeno. Esto indica que el oxígeno se utiliza para la oxidación del hidrocarburo, de forma que decae la conversión de los óxidos de nitrógeno. La conversión de $\mathrm{NO}_{x}$ baja más cuanto más oxígeno entra en la alimentación, lo que indica que hay competencia entre los oxidantes, produciéndose la combustión del propeno a expensas de oxígeno y sin que haya reacción con el $\mathrm{NO}_{x}$.

Si se utiliza oxígeno como único oxidante, en condiciones estequiométricas, se observa la conversión total del hidrocarburo para todos los catalizadores, independientemente de la forma de incorporación del cromo a los catalizadores y de la cantidad de metal que contienen. Se observa que la combustión comienza sobre los $200 \stackrel{\circ}{ } \mathrm{C}$, y a partir de esta temperatura aumenta bruscamente, y en todos los casos alcanza el $100 \%$ a 400 C. Por tanto, la oxidación del propeno está muy vinculada a la presencia de cromo en los catalizadores, sin ser determinantes la posición del cromo ni las propiedades superficiales de los catalizadores.

Cabe señalar que en ningún caso se observó la formación de otros compuestos nitrogenados, dentro del límite de detección de la técnica utilizada, indicando que el nitrógeno es el único producto de la reacción de reducción del óxido de nitrógeno. 
La impregnación con sales de níquel de la saponita pilareada con aluminio permitió conservar la estructura laminar de los sólidos, así como elevados valores de superficie específica y microporosidad, al tiempo que se incorporaban cantidades variables de este elemento.

Estos sólidos también fueron utilizados en la reacción de reducción de óxidos de nitrógeno. Los únicos productos detectados en los efluentes de la reacción fueron $\mathrm{N}_{2}$ y $\mathrm{CO}_{2}$, observándose también depósitos carbonosos. Las conversiones de propeno alcanzaron porcentajes de hasta un $30 \%$, mientras que para el soporte son siempre inferiores al 5\%, confirmando la importancia de la incorporación de níquel. Por su parte, la reducción de óxidos de nitrógeno alcanza valores del 19\%. Estos resultados son similares a los encontrados utilizando otros catalizados basados en níquel, y sugieren que las especias carbonosas formadas durante la reacción catalítica son las principales responsables de la baja actividad.

El factor que más afecta al comportamiento de los catalizadores estudiados es la naturaleza del precursor utilizado para incorporar el níquel al soporte, siendo el sólido preparado a partir del acetilacetonato el más activo. La cantidad de níquel incorporado influye en mucha menor medida. La actividad está relacionada con la presencia de especies $\mathrm{NiO}$ y $\mathrm{NiAl}_{2} \mathrm{O}_{4}$, aunque la presencia de centros ácidos también es relevante. La presencia de níquel formando parte de aluminatos confiere menor poder reductor que el que presentan las partículas de óxido másico, y el mejor comportamiento catalítico del sólido preparado con acetilacetonato de níquel puede justificarse por la presencia de NiO, además de $\mathrm{NiAl}_{2} \mathrm{O}_{4}$, en su superficie. De hecho, la deconvolución de las curvas TPR de estos sólidos permitió identificar tres efectos de reducción de níquel, cuyas temperaturas e intensidades relativas variaron en cada muestra.

Comparando la reacción al utilizar la mezcla $\mathrm{NO}_{\mathrm{x}}-\mathrm{O}_{2}$ y al utilizar $\mathrm{O}_{2}$ como único oxidante, se observa que todos los catalizadores preparados presentan una reducción selectiva de los óxidos de nitrógeno a temperaturas intermedias. En todos los casos, la oxidación de hidrocarburo a expensas del oxígeno se ve favorecida a temperaturas por encima de los $400 \stackrel{\circ}{ } \mathrm{C}$, mientras que a temperaturas inferiores la oxidación se produce utilizando el óxido de nitrógeno como oxidante. 
Como vías alternativas para la preparación de catalizadores de níquel se utilizaron los métodos sol-gel y de co-precipitación (los detalles experimentales se ofrecen en el próximo capítulo). Aunque se buscaron condiciones que podrían conducir a la síntesis de saponita, ésta no se consiguió, y en el caso del método sol-gel se obtuvo una mezcla de sílice amorfa y $2 \mathrm{NaAlO}_{2} \cdot 3 \mathrm{H}_{2} \mathrm{O}$, mientras que en el caso de la co-precipitación se obtuvo un nanocomposite formado por sílice amorfa y takovita, la hidrotalcita de Ni-Al. La calcinación de estos sólidos a diferentes temperaturas permitió obtener interesantes catalizadores de níquel que, junto con los sólidos obtenidos anteriormente por impregnación de níquel sobre saponita pilareada, fueron utilizados en reacciones de oxidación de hidrocarburos, en concreto la epoxidación de (Z)-cicloocteno y la oxidación de ciclohexano.

El sólido seco obtenido mediante el método sol-gel da lugar a una conversión de (Z)-cicloocteno del 100\%, siendo el epóxido el único producto de la reacción. La conversión del alqueno varía bruscamente en función de la temperatura de calcinación de los sólidos. Desde el $100 \%$ del sólido seco, la conversión disminuye al 49 y $14 \%$ para los sólidos calcinados a 300 y 450 드, respectivamente, volviendo a aumentar para los sólidos tratados a 600 y 1000 C, alcanzándose valores del $35 \%$. La diferente actividad de los catalizadores preparados puede justificarse por la presencia de níquel en diferentes fases, así como por las diferentes propiedades texturales de los sólidos obtenidos. La muestra seca tiene cationes $\mathrm{Al}^{3+} \mathrm{y} \mathrm{Ni}^{2+}$ (ambos activos para la epoxidación del cicloocteno) ocupando posiciones octaédricas en las láminas, facilitando la accesibilidad de la olefina a dichos cationes, lo que se ve favorecido por el hecho de que los sólidos estén bastante deslaminados, es decir, no presenten una ordenación de largo alcance en el eje c.

En el caso de las muestras preparadas por co-precipitación, la conversión de alqueno es mucho menor, entre el 20 y el 35\%. Estos sólidos están compuestos por fases tipo hidrotalcita y sílice, por lo que parece que las posiciones ocupadas por $\mathrm{Al}^{3+}$ y $\mathrm{Ni}^{2+}$ en estos sólidos son mucho menos activas que en los obtenidos mediante el método sol-gel. De hecho, los sólidos obtenidos mediante coprecipitación tienen mejores propiedades texturales, sugiriendo que las posiciones de los metales son determinantes en la actividad catalítica de los sólidos.

Los catalizadores preparados por impregnación de sales de níquel sobre saponita pilareada con aluminio también mostraron un excelente comportamiento 
en estas reacciones. En la epoxidación del cicloocteno, se alcanzan conversiones del $30 \%$, con total selectividad al epóxido, mientras que en la oxidación de ciclohexano la conversión también alcanza el 30\%, siendo destacable que no se formó ciclohexanol, la selectividad fue total hacia la ciclohexanona. Esto puede deberse a que el carácter ácido del soporte arcilloso aumenta la formación de especies metálicas activas, favoreciendo que la reacción vaya hacia el producto más polar, la cetona. Estos datos, junto a los obtenidos en experimentos adicionales utilizando secuestradores de radicales, indican que la transferencia de oxígeno a los substratos es llevada a cabo por especies de níquel de alta valencia, por lo que los catalizadores utilizados se comportan de manera análoga a sistemas biológicos enzimáticos.

\section{I.12.2. Indicios de calidad de las revistas en las que se han publicado los resultados de esta Tesis Doctoral}

Como se ha indicado en el apartado anterior, los resultados obtenidos en la presente Tesis Doctoral han sido publicados en cuatro artículos científicos y en un capítulo de libro. A continuación se comentan los indicios de calidad de las revistas en las que se han publicado estos resultados.

El criterio de calidad más utilizado por la comunidad científica internacional para clasificar las revistas especializadas es el factor de impacto (FI). Este índice, elaborado anualmente, es una medida de la frecuencia con la que un artículo de una revista ha sido citado en dicho año en el conjunto de las revistas incluidas en el sistema. De esta manera, se establece una clasificación relativa de las revistas, al comparar las pertenecientes al mismo campo científico. Numéricamente, el FI de una revista en un año determinado se calcula como el cociente entre el número total de citaciones recibidas en ese año por los artículos publicados en la revista en los dos años anteriores al mismo y el número total de artículos publicados en dicha revista al cabo del mismo periodo.

De esta manera, Thomson Reuters elabora anualmente el Journal Citation Reports ${ }^{\circledR}$, clasificando más de 8.000 revistas en el área de Ciencia y Tecnología. Como se ha comentado anteriormente, estas revistas se sitúan en diferentes 
campos científicos, pudiendo estar una misma revista clasificada en varios campos científicos según sus líneas de investigación prioritarias.

En la Tabla I.2 se presentan los $\mathrm{Fl}$ de los últimos 5 años de las revistas en las que se han publicado los resultados derivados de esta Tesis Doctoral, y su respectiva posición dentro de las categorías en las que se encuentran clasificadas.

\begin{tabular}{|c|c|c|c|c|c|}
\hline Año $\rightarrow$ & 2007 & 2008 & 2009 & 2010 & 2011 \\
\hline \multicolumn{6}{|c|}{ Applied Catalysis A: General } \\
\hline Factor de impacto & 3,166 & 3,190 & 3,564 & 3,384 & 3,903 \\
\hline Clasificación relativa: & & & & & \\
\hline Química Física & $26 / 110$ & $29 / 113$ & $30 / 121$ & $35 / 127$ & $31 / 134$ \\
\hline Ciencias Medioambientales & $14 / 160$ & $21 / 163$ & $18 / 181$ & 23/193 & $20 / 205$ \\
\hline \multicolumn{6}{|c|}{ Catalysis Letters } \\
\hline Factor de impacto & 1,883 & 1,867 & 2,021 & 1,907 & 2,242 \\
\hline $\begin{array}{l}\text { Clasificación relativa: } \\
\text { Química Física }\end{array}$ & $52 / 110$ & $56 / 113$ & $59 / 121$ & $72 / 127$ & $60 / 134$ \\
\hline \multicolumn{6}{|c|}{ Microporous and Mesoporous Materials } \\
\hline Factor de impacto & 2,699 & 2,555 & 2,652 & 3,220 & 3,285 \\
\hline Clasificación relativa: & & & & & \\
\hline Química Aplicada & $12 / 62$ & $9 / 61$ & $8 / 64$ & $5 / 70$ & $8 / 71$ \\
\hline Química Física & $40 / 110$ & $38 / 113$ & $41 / 121$ & $38 / 127$ & $40 / 134$ \\
\hline Nanociencia y Nanotecnología & $14 / 46$ & $16 / 52$ & $22 / 59$ & $23 / 64$ & $25 / 66$ \\
\hline $\begin{array}{l}\text { Ciencia de los Materiales } \\
\text { (multidisciplinar) }\end{array}$ & 29/189 & $31 / 192$ & $35 / 214$ & $37 / 125$ & $39 / 231$ \\
\hline
\end{tabular}

Tabla I.2. Factor de impacto (FI) en los últimos cinco años de las revistas en las que se han publicado los resultados de esta Tesis Doctoral, y clasificación relativa en las categorías en las que se encuentran incluidas (Tomado del J CR ${ }^{8}$ ).

La revista Applied Catalysis A: General, publicada por la editorial Elsevier, cubre todos los aspectos relevantes de la catálisis homogénea y heterogénea, $y$, en menor medida, de la preparación y caracterización de catalizadores. Su factor de impacto es elevado, siendo claramente superior a 3,0, y acercándose a 4,0, en los últimos años. Ha estado tradicionalmente incluida en la categoría de Química Física, en la cual fluctúa entre el primer y segundo cuartil. En los últimos años, se ha 
incorporado a la categoría de Ciencias Medioambientales, en la cual está situada en el primer cuartil y se ha convertido en una revista de referencia internacional.

Catalysis Letters, publicada por la editorial Springer, está igualmente dirigida a cubrir todos los ámbitos de la catálisis. Su factor de impacto en los últimos años se ha situado en torno a 2,0, aunque el prestigio de la revista en la comunidad científica es mayor que el indicado por este índice de impacto. Se encuentra clasificada únicamente en la categoría de Química Física, en la que fluctúa entre el segundo y el tercer cuartil. Por tanto, puede considerarse una revista de nivel medio-alto en dicha categoría.

Por su parte, Microporous and Mesoporous Materials, también publicada por la editorial Elsevier, está enfocada al estudio de todo tipo de sólidos porosos, cubriendo su preparación, caracterización, estudios teóricos y de modelización, y particularmente sus aplicaciones, en adsorción, catálisis, electroquímica, membranas, sensores, etc. Debido a la variedad de temas abordados, se encuentra incluida en cuatro categorías, Química Aplicada, Química Física, Nanociencia y Nanotecnología, y Ciencia de los Materiales. Su factor de impacto es elevado, habiendo superado el valor de 3,2 en las dos últimas clasificaciones disponibles. Con esto, se sitúa en el segundo cuartil en las categorías de Química Física y de Nanociencia y Nanotecnología, y en el primer cuartil en las otras categorías en las que se encuentra incluida, por lo que se puede considerar también una revista de primer nivel en el campo de la Ciencia de los Materiales.

Por todo ello, puede indicarse que los resultados obtenidos en esta Tesis Doctoral han sido divulgados en revistas que presentan un alto factor de impacto y una categoría ampliamente reconocida en los ámbitos de la Catálisis y la Ciencia de los Materiales.

\section{I.13. Bibliografía}

[1] Grim R.E. Clay Mineralogy. McGraw-Hill Book Company. Nueva York (1953).

[2] Gomes C. Argilas. O que são e para que servem. Fundação Calouste Gulbenkian. Lisboa (1988). 
[3] Velde B. (Editor). Origin and Mineralogy of clays: Clays and the Environment. Springer. Berlín (1995).

[4] Nesse W.D. Introduction to Mineralogy. Oxford University Press. Oxford (2000).

[5] Hofmann U., Endell K., Wiem D. Struktur und quellung von montmorillonit, das tonmineral der bentonite. Zeitschrift für Kristallographie 86, 340-347 (1933).

[6] De la Calle C., Suquet H. Vermiculite. En: Hydrous phyllosilicates. S.W. Bailey (Ed.). Mineralogical Society of America, Reviews in Mineralogy 19, 455-496, Washington (1988).

[7] Thorez J. Practical Identification of Clay Minerals. A Handbook for Teachers and Students in Clay Mineralogy. Editions G. Lelotte, Dison (1978).

[8] Velde B. Introduction to Clay Minerals. Chemistry, Origins, Uses and Environmental Significance. Chapman \& Hall (1992).

[9] Bergaya F., Lagaly G., Vayer M. Cation and Anion Exchange. En: Handbook of Clay Science, Bergaya F., Theng B.K.G., Lagaly G. (Eds.). Developments in Clay Science. Elsevier Ltd. Amsterdam (2006).

[10] Gast R.G. Surface and Colloid Chemistry. En: Minerals in Soil Environments. Dixon J.B., Weed S.B. (Eds.). Soil Science Society of America. Madison (1977).

[11] Anderson S.J., Sposito G. Cesium-adsorption method for measuring accessible structural surface charge. Soil Science Society of America Journal, 55, 15691576 (1991).

[12] Moore D.M., Reynolds R.C. Jr. X-Ray Diffraction and the Identification and Analysis of Clay Minerals. 2nd. ed. Oxford University Press. New York (1997).

[13] Adams J.M., McCabe R.W. Clay Minerals as Catalysts. En: Handbook of Clay Science, Bergaya F., Theng B.K.G., Lagaly G. (Eds.). Developments in Clay Science. Elsevier Ltd. Amsterdam (2006).

[14] Gil A., Korili S.A., Trujillano R., Vicente M.A. (Eds.), Pillared Clays and Related Catalysts. Springer. New York-Dordrecht-Heidelberg-London (2010). 
[15] Bergaya F., Aouad A., Mandalia T. Pillared Clays and Clay Minerals. En: Handbook of Clay Science, Bergaya F., Theng B.K.G., Lagaly G. (Eds.). Developments in Clay Science. Elsevier Ltd. Amsterdam (2006).

[16] Gil A., Gandía L.M., Vicente M.A.. Recent advances in the Synthesis and catalytic applications of pillared clays. Catalysis Reviews-Science and Engineering 42, 145-212 (2000).

[17] Ertl G., Knözinger H., Schüth F., Weitkamp J. Handbook of Heterogeneous Catalysis, 2nd edition. Wiley-VCH (2008).

[18] Brinker C.J., Scherer G.W. Sol-Gel Science, The Physics and Chemistry of SolGel Processing. Academic Press (1990).

[19] Piccaluga G., Corrias A., Ennas G., Musinu A. Sol-Gel Preparation and Characterization of Metal-Silica and Metal Oxide-Silica Nanocomposites, Trans Tech Publications (2000).

[20] Wright J.D., Sommerdijk N.A.J.M. Sol-Gel Materials Chemistry and Applications, Taylor \& Francis (2000). 



\section{Capítulo I I}

Materiales de partida y métodos experimentales 



\section{I.1. Materiales de partida}

La arcilla utilizada para realizar este trabajo es una saponita de origen natural, procedente del yacimiento de Yunclillos-Cabañas, Toledo (se han utilizado dos muestras extraídas en dos puntos diferentes, pero ambas pertenecen al mismo yacimiento). La mina es explotada por la empresa TOLSA (Madrid).

La empresa suministradora proporciona la saponita tal como es extraída del yacimiento, molida y seca, pero sin ningún proceso de purificación, por lo que contiene diversas impurezas mineralógicas identificables mediante difracción de rayos $\mathrm{X}$, como cuarzo y feldespatos, así como trazas de sepiolita y mica, $\mathrm{y}$ pequeñas cantidades de materia orgánica (Figura II.1).

La purificación del material de partida se llevó a cabo por decantación atendiendo a la definición de arcilla como fracción del suelo con tamaño de partícula menor de dos micras. Dado que las partículas arcillosas constituyen la fracción más pequeña y menos densa de cualquier suelo, se puede aprovechar esta propiedad para extraerlas y realizar así la purificación. Para llevar a cabo la separación, se tiene en cuenta la diferente velocidad de caída de las partículas dispersadas en el seno de un fluido, en función de su tamaño y densidad. Matemáticamente, esta velocidad viene dada por la ley de Stokes (ecuación II.1):

$$
v=\frac{2}{9 \eta} r^{2} g\left(\rho_{P}-\rho_{S}\right)
$$

Ecuación II.1. Ley de Stokes $(v=$ velocidad de sedimentación de una partícula en el seno de un líquido; $r=$ radio de la partícula; $n=$ viscosidad de la suspensión; $\mathrm{g}=$ aceleración de la gravedad; $\rho_{\mathrm{p}}$ y $\rho_{\mathrm{S}}=$ densidad de la partícula y de la suspensión, respectivamente). Al trabajar con una suspensión diluida de arcilla en agua, los valores de $\eta$ y $\rho_{\mathrm{s}}$ se asimilan a los del líquido.

Según esta ecuación las partículas descenderán en el seno de la suspensión en función de sus propiedades físicas, las de mayor tamaño y densidad caen al fondo del fluido en un tiempo menor que las pequeñas y ligeras. Al aplicarla a partículas arcillosas suspendidas en agua, con un diámetro de $2 \mu \mathrm{m}$ y densidad de $2,2 \mathrm{~g} / \mathrm{cm}^{3}$ (densidad típica de las partículas arcillosas), se obtiene una velocidad de sedimentación de 1,25 cm/hora. Con esta velocidad podemos calcular qué altura de columna de suspensión se ve enriquecida en la fracción arcilla para un tiempo 
determinado, dicha altura de columna puede extraerse realizando así la purificación del material de partida.

Experimentalmente, esta purificación se lleva a cabo preparando una suspensión de material arcilloso dispersando unos $50 \mathrm{~g}$ de arcilla en $800 \mathrm{~mL}$ de agua, se agita y se observa su dispersabilidad. Debido a la presencia de pequeñas cantidades de materia orgánica, es necesario añadir unas gotas de amoníaco como agente dispersante para que la dispersión sea correcta. Una vez que se consigue una completa dispersión de la muestra, la suspensión se mantiene en agitación durante una noche, tras la cual se deja decantar para que la parte superior de la columna se enriquezca en fracción arcilla, y poder proceder a su extracción por sifonado. En nuestro caso, se dejó un tiempo de decantación de ocho horas, que, según la ley de Stokes, asegura que todas las partículas de tamaño mayor a dos micras han caído por debajo de la altura extraída, mientras que los $10 \mathrm{~cm}$ superiores de la columna de fluido estarán constituidos únicamente por la fracción arcillosa, por lo que se extrae esta parte de la columna.

La suspensión obtenida se centrifuga, la arcilla purificada se seca en una estufa a $70{ }^{\circ} \mathrm{C}$ y se muele en mortero de ágata, quedando el sólido así obtenido listo para su posterior tratamiento. Los difractogramas de rayos $X$ (Figura II.1) confirman la eficacia de la purificación, habiendo disminuido en gran medida las impurezas mineralógicas presentes en la arcilla de partida.

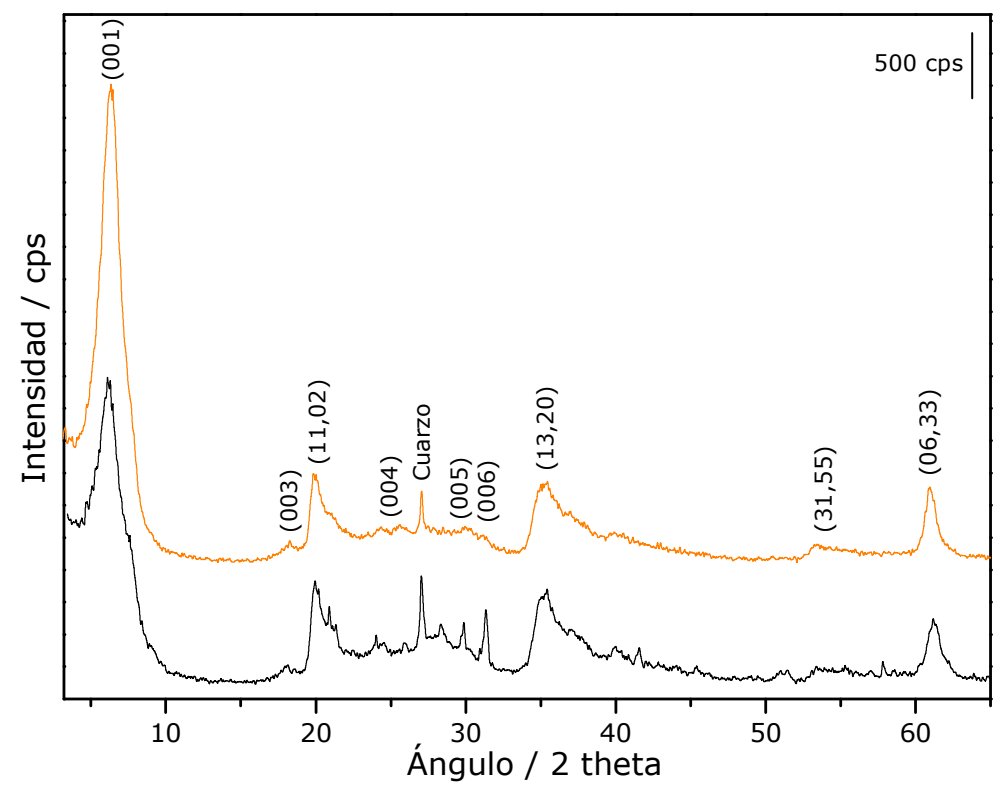

Figura II.1. Difractogramas de rayos $X$ de la saponita de Yunclillos antes (abajo) y después (arriba) de la purificación. 


\section{I.2. Preparación de los catalizadores}

Una vez obtenida la fracción arcilla del tamaño de partícula deseado, se procede a los tratamientos necesarios para realizar las modificaciones químicas anteriormente indicadas, esto es, intercalación, cointercalación e impregnación, respectivamente.

La arcilla de partida fue intercalada con disoluciones de policationes de aluminio y cromo, y cointercalada con distintas relaciones de ambos metales. Posteriormente, se realizó la pilarización de las muestras intercaladas. Además, la saponita pilarizada con aluminio se impregnó posteriormente con diferentes sales de cromo, para de esta forma estudiar la influencia del método de incorporación del metal a la arcilla en la actividad catalítica de los sólidos finales.

Dado que el níquel no presenta la posibilidad de formación de policationes, ni se ha descrito su sustitución isomórfica en el policatión $\mathrm{Al}_{13}$, la preparación de los catalizadores de níquel se realiza únicamente mediante la impregnación de la saponita previamente pilareada con policationes de aluminio.

\section{II.2.1. Modificaciones químicas de la saponita}

\section{I.2.1.a. I ntercalación y pilarización}

Como se ha indicado, la saponita ha sido intercalada con policationes de Al, $\mathrm{Cr}$ y policationes mixtos $\mathrm{Al} / \mathrm{Cr}$. El primer paso para realizar el proceso de intercalación y pilarización es la preparación de las disoluciones intercalantes. Esta preparación varía en función de la relación entre metales, serán necesarios diferentes tiempos, tanto de envejecimiento de los policationes como de contacto policatión-arcilla, para que tenga lugar el proceso, como se detalla a continuación. Dado que la intercalación de la saponita se realiza mediante intercambio catiónico, se ponen en contacto las disoluciones intercalantes preparadas con suspensiones acuosas de arcilla purificada. Tras un tiempo de contacto, los sólidos se centrifugan, se lavan y se secan para después proceder a su pilarización. 
Experimentalmente, las disoluciones intercalantes se preparan mediante la disolución de las correspondientes sales metálicas, empleando las cantidades deseadas de cada uno de los cationes e hidrolizando con una disolución de hidróxido sódico. Como fuentes de los metales se han utilizado sales hidratadas solubles en agua, $\mathrm{AlCl}_{3} \cdot 6 \mathrm{H}_{2} \mathrm{O}$ y $\mathrm{CrCl}_{3} \cdot 6 \mathrm{H}_{2} \mathrm{O}$, respectivamente. Se prepararon disoluciones intercalantes de composición $\mathrm{Al}_{x} \mathrm{Cr}_{y}$, donde $\mathrm{x}$ e y son, respectivamente, las fracciones molares de aluminio y cromo en el seno de la disolución preparada. Así, la disolución $\mathrm{Al}_{1,0}$ contiene únicamente la sal de aluminio, mientras que la $\mathrm{Cr}_{1,0}$ está formada sólo por la de cromo. El proceso general para la obtención de sólidos intercalados y pilareados se resume en la Figura II.2, detallándose más adelante las diferencias en las condiciones de síntesis de cada uno de los catalizadores en función de la relación de metales $\mathrm{Al} / \mathrm{Cr}$ que contengan.

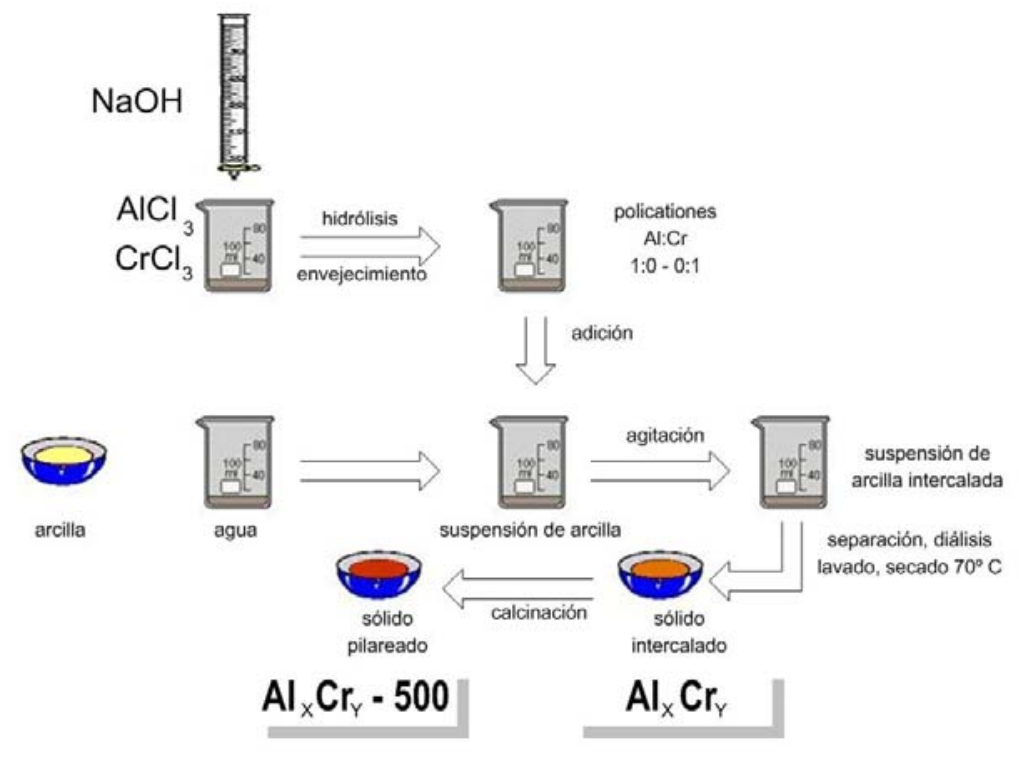

Figura II.2. Esquema de la preparación de los sólidos intercalados y pilareados.

La disolución intercalante $\mathrm{Al}_{1,0}$ fue preparada según el método descrito en la bibliografía [1-3], para lo cual se disuelve la cantidad de sal de aluminio necesaria y se hidroliza mediante la adición lenta de una disolución básica; la cantidad de la disolución básica ha de ser la adecuada para obtener una relación $\mathrm{OH}^{-} / \mathrm{Al}^{3+}$ de 2,2. Para ello, se prepara una disolución de 30 mmoles de $\mathrm{AlCl}_{3} \cdot 6 \mathrm{H}_{2} \mathrm{O}$ en $140 \mathrm{~mL}$ de agua bidestilada, sobre la que se añade una segunda disolución que contiene 66 mmoles de $\mathrm{NaOH}$, preparada por dilución de $66 \mathrm{~mL}$ de $\mathrm{NaOH} 1 \mathrm{M}$ en $245 \mathrm{~mL}$ de agua. Para realizar la hidrólisis se debe adicionar la disolución alcalina muy lentamente y con agitación fuerte de forma que se eviten altas concentraciones locales de hidroxilo, que pueden dar lugar a la precipitación de hidróxido de 
aluminio. Para favorecer la polimerización del catión, la disolución se deja envejecer durante 24 horas. En la disolución obtenida en estas condiciones, la mayoría del aluminio estará en forma de policatión de Keggin $\left[\mathrm{Al}_{13} \mathrm{O}_{4}(\mathrm{OH})_{24}\left(\mathrm{H}_{2} \mathrm{O}\right)_{12}\right]^{7+}$.

La preparación de la disolución $\mathrm{Cr}_{1,0}$ se llevó a cabo disolviendo la cantidad de sal de cromo necesaria para obtener 30 mmoles de metal en $140 \mathrm{~mL}$ de agua. La relación $\mathrm{OH}^{-} / \mathrm{Cr}^{3+}$ desciende en este caso hasta 2,0 [4], por lo que es necesaria una disolución que contenga 60 mmoles de grupos hidroxilo, que se prepara mediante dilución de $60 \mathrm{~mL}$ de $\mathrm{NaOH} 1 \mathrm{M}$ en $245 \mathrm{~mL}$ de agua. Esta disolución se adiciona lentamente y con agitación fuerte sobre la disolución de $\mathrm{Cr}^{3+}$. Para favorecer la formación de policationes, la disolución se deja envejecer, en reposo pero con agitación ocasional, durante 72 horas.

Las disoluciones mixtas, compuestas por distintas relaciones de $\mathrm{Al} / \mathrm{Cr}$, se preparan mediante disolución de las sales de ambos metales, en la forma descrita en la bibliografía para otras mezclas de metales [3]. Se prepararon disoluciones intercalantes que contenían las cantidades de $\mathrm{Al}$ y $\mathrm{Cr}$ indicadas en la Tabla II.1, de manera que la cantidad total de cationes es siempre 30 mmoles. La hidrólisis de cada una de las disoluciones se realiza con cantidades de $\mathrm{NaOH}$ que varían en función de la cantidad de cada uno de los metales, de manera que se aproximan a las disoluciones intercalantes simples de aluminio o de cromo según su composición sea más próxima a una u otra. Los tiempos de envejecimiento también se varían en función de la relación de metales que componen la disolución. Las cantidades de cada metal utilizadas para cada una de las disoluciones, junto con las condiciones de preparación y su nomenclatura se detallan en la Tabla II.1.

\begin{tabular}{|c|c|c|c|c|}
\hline $\begin{array}{l}\text { Disolución } \\
\text { intercalante }\end{array}$ & $\begin{array}{c}\mathbf{m m o l} \\
\mathrm{Al}^{3+}\end{array}$ & $\begin{array}{c}\mathrm{mmol} \\
\mathrm{Cr}^{3+}\end{array}$ & $\begin{array}{l}\text { Relación } \\
\mathrm{OH}^{-} / \mathrm{Me}^{3+}\end{array}$ & $\begin{array}{c}\text { Tiempo de } \\
\text { envejecimiento }\end{array}$ \\
\hline $\mathrm{Al}_{1,0}$ & 30 & 0 & 2,2 & 24 horas \\
\hline $\mathrm{Al}_{0,9} \mathrm{Cr}_{0,1}$ & 27 & 3 & 2,2 & 24 horas \\
\hline $\mathrm{Al}_{0,5} \mathrm{Cr}_{0,5}$ & 15 & 15 & 2,1 & 48 horas \\
\hline $\mathrm{Al}_{0,2} \mathrm{Cr}_{0,8}$ & 6 & 24 & 2,0 & 72 horas \\
\hline $\mathrm{Cr}_{1,0}$ & 0 & 30 & 2,0 & 72 horas \\
\hline
\end{tabular}

Tabla II.1. Condiciones empleadas para la obtención de $6 \mathrm{~g}$ de arcilla intercalada, y nomenclatura de las disoluciones utilizadas. 
Una vez realizada la intercalación, se obtiene una suspensión de arcilla que se separa mediante centrifugación y se lava. El proceso de lavado es necesario para eliminar los contraiones de las sales utilizadas como fuentes de metales, los policationes que no se han situado en el espacio interlaminar y los cationes de cambio de la arcilla natural que han sido sustituidos por policationes en el proceso de intercalación. Dicho lavado se lleva a cabo suspendiendo de nuevo la arcilla en la menor cantidad posible de agua (100-150 mL), la suspensión obtenida se introduce en una membrana de diálisis y se cierra, y dicha membrana se introduce a su vez en un recipiente que contiene $3 \mathrm{~L}$ de agua bidestilada. El agua de lavado se renueva cada 12 horas hasta que se consigue la total eliminación del exceso se iones, lo que se comprueba analizando cualitativamente los iones $\mathrm{Cl}^{-}$con una disolución $1 \mathrm{M}$ de $\mathrm{AgNO}_{3}$. Al añadir unas gotas de esta disolución a una alícuota de las aguas de lavado comprobamos la presencia de cloruros por la aparición de un precipitado blanquecino de $\mathrm{AgCl}[5]$, el lavado continúa hasta que esta prueba resulta negativa. Completado el lavado, los sólidos se centrifugan y se secan en una estufa a $70{ }^{\circ} \mathrm{C}$, obteniéndose así los sólidos intercalados, que recibieron los mismos nombres que las disoluciones a partir de las que se obtienen.

El proceso de pilarización se lleva a cabo mediante la calcinación de los sólidos intercalados. La calcinación se realiza en atmósfera de aire, utilizando una rampa de temperatura de $1^{\circ} \mathrm{C} / \mathrm{min}$; el aumento lento y paulatino de temperatura se utiliza para favorecer la deshidroxilación lenta de los policationes intercalados, evitando así la formación rápida de clusters metálicos a partir de los policationes intercalados y el colapso de la estructura laminar. Se mantiene la rampa de calentamiento hasta alcanzar los $500^{\circ} \mathrm{C}$, temperatura que se mantiene durante dos horas. Esta temperatura fue elegida porque es la habitualmente utilizada para la preparación de arcillas pilareadas con aluminio y porque será la máxima utilizada para realizar la reacción catalítica de reducción de NOx. Una vez terminado el proceso de calcinación, el sólido se deja enfriar y obtenemos la arcilla pilarizada. La nomenclatura de los sólidos pilareados es el nombre de los sólidos intercalados de partida seguido de la temperatura de calcinación: $\mathrm{Al}_{x} \mathrm{Cr}_{y}-500$. 


\section{I.2.1.b. I mpregnación}

\section{II.2.1.b.1. Muestras impregnadas con cromo}

La arcilla pilarizada con aluminio en el proceso anterior, $\mathrm{Al}_{1,0}-500$, se utilizó como soporte para la incorporación de cromo mediante la técnica de impregnación a humedad incipiente. Este proceso consiste en añadir a una determinada cantidad de soporte seco el volumen necesario de una disolución de sal metálica para que quede completamente humedecido, pero sin disolución sobrante. La disolución se añade lentamente, mezclándola cuidadosamente con el soporte, favoreciendo así que se introduzca por capilaridad en los poros del mismo, eliminando el aire inicialmente presente en ellos, que queda sustituido por la disolución metálica. En la Figura II.3 se muestra un esquema del proceso.

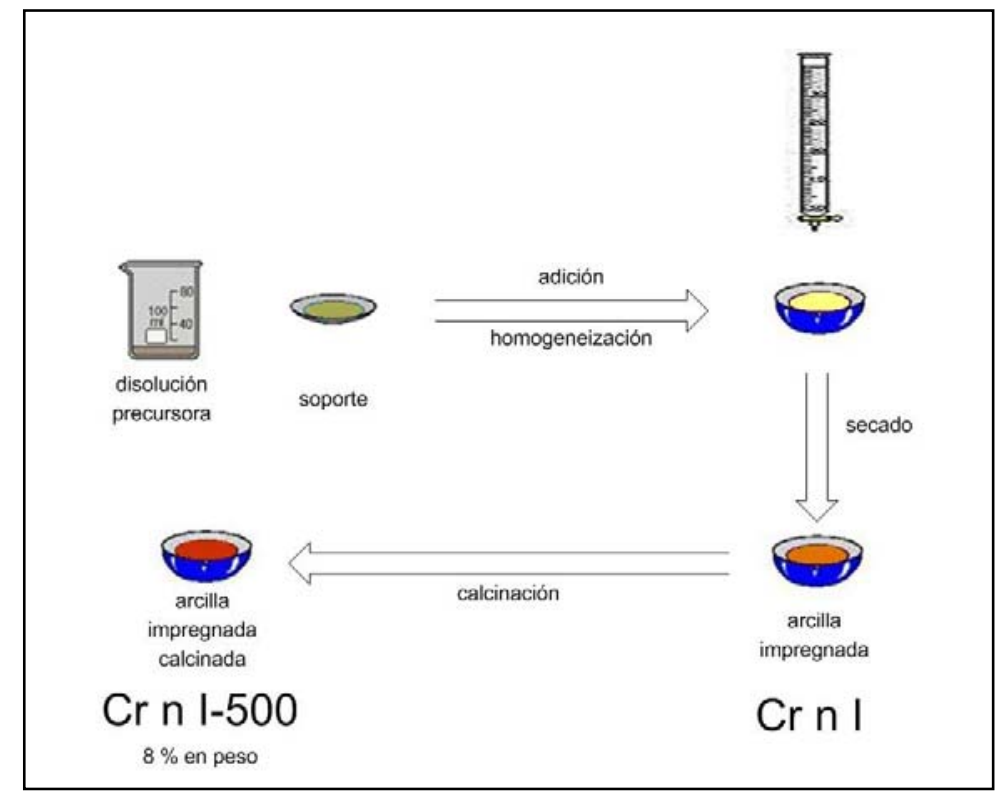

Figura II.3. Esquema de la preparación de los sólidos impregnados.

Para preparar los sólidos impregnados debemos calcular el volumen de retención del soporte, es decir, cuál es el volumen de disolución que admite una determinada masa de sólido pilareado, sin que quede exceso de disolución. Para ello, humedecemos la arcilla con los disolventes que utilizaremos para disolver los precursores, en este caso, acetona y agua. Se observó que son necesarios 16 y $9 \mathrm{~mL}$ de acetona y agua, respectivamente, para realizar la impregnación de $5 \mathrm{~g}$ de soporte. A continuación, se calcula la cantidad necesaria de cada uno de los precursores considerados para obtener un contenido de $\mathrm{Cr}_{2} \mathrm{O}_{3}$ en los sólidos finales 
del $8 \%$ en peso. Se consideraron precursores con $\mathrm{Cr}$ en distintos estados de oxidación, uno de $\mathrm{Cr}(\mathrm{II})$, dos de $\mathrm{Cr}$ (III) y uno de $\mathrm{Cr}(\mathrm{VI})$, como se recoge en la Tabla II.2. Se disolvió la cantidad necesaria de cada uno de ellos en el disolvente adecuado y se procedió a la impregnación del soporte según el proceso descrito anteriormente. En todos los casos, las cantidades necesarias de los distintos precursores se disolvieron sin dificultad en $9 \mathrm{~mL}$ de agua o $16 \mathrm{~mL}$ de acetona, por lo que la impregnación se realizó en un único ciclo. Los catalizadores impregnados preparados permitirán analizar las diferencias de actividad en función del estado de oxidación inicial del metal incorporado, y la posible influencia de los disolventes utilizados para la impregnación.

\begin{tabular}{|c|c|c|}
\hline Nomenclatura & Precursor & Disolvente \\
\hline Cr1 I & Acetato de Cr(II) & Agua \\
Cr2 I & Acetilacetonato de Cr(III) & Acetona \\
Cr3 I & Cromato amónico & Agua \\
Cr4 I & Nitrato de Cr(III) & Agua \\
\hline
\end{tabular}

Tabla II.2. Reactivos utilizados para la preparación de los sólidos impregnados con cromo, y nomenclatura de los sólidos resultantes.

Una vez realizada la impregnación, los sólidos se dejan secar a temperatura ambiente durante dos días, y finalmente se calcinan en atmósfera de aire para obtener los catalizadores metálicos deseados. La calcinación se lleva a cabo en una mufla, en atmósfera de aire, con una rampa de calentamiento de $1^{\circ} \mathrm{C} / \mathrm{min}$ desde temperatura ambiente hasta $500{ }^{\circ} \mathrm{C}$, manteniendo esta temperatura final durante dos horas. En este caso, como la arcilla utilizada como soporte ya está pilareada, la velocidad lenta se usa para eliminar progresivamente los ligandos y contraiones de las sales de partida y evitar el colapso de las láminas de arcilla que puede producir un aumento rápido de la temperatura. La nomenclatura dada a los catalizadores resultantes tras el proceso de secado es la indicada en la Tabla II.2, mientras que para los sólidos calcinados se añadió a dichos nombres la temperatura de calcinación: Cr n I-500.

Además de las anteriores, se realiza una quinta impregnación sobre una alúmina comercial (alúmina esferalita 505) usando como precursor nitrato de cromo (III) en las condiciones de preparación descritas, para poder comprobar si el soporte influye en la actividad de los catalizadores. 


\section{II.2.1.b.2. Muestras impregnadas con níquel}

La preparación de catalizadores arcillosos basados en níquel, debido a que éste no ofrece la posibilidad de formación de policationes ni sustituciones en el policatión de Keggin, se realiza por impregnación de arcilla pilareada. Para la preparación de estos catalizadores se utilizó la saponita de Cabañas como arcilla inicial, que fue pilareada con policationes $\mathrm{Al}_{13}$ según el procedimiento descrito en el apartado anterior y, como se ha indicado, utilizada como soporte.

La impregnación se llevó a cabo, como en el caso anterior, por el método de humedad incipiente. El sólido precursor (la saponita pilareada) se humedeció con disoluciones que contenían las cantidades deseadas del níquel, de forma que el sólido quedara completamente humedecido pero sin disolución sobrante. Para llevar a cabo esta impregnación se determinó previamente qué volumen de cada disolución era necesario para mojar el sólido, para lo que se humedeció la arcilla pilareada con agua y acetona (los dos disolventes utilizados en la impregnación), determinando el volumen a utilizar en cada uno de los ciclos.

Las impregnaciones se realizaron utilizando cloruro de níquel (II), nitrato de níquel (II) y acetilacetonato de níquel (II) como sales precursoras para obtener las cantidades deseadas de fase activa (Tabla II.3). Se prepararon catalizadores soportados que contienen el $1,5,3,0,4,5$ y $6,0 \%$ de fase activa (referida en todos los casos a óxido de níquel, $\mathrm{NiO})$.

\begin{tabular}{|c|c|c|}
\hline Nomenclatura & Precursor & Disolvente \\
\hline $\mathrm{NiCl}$ & Cloruro de Ni(II) & Agua \\
$\mathrm{Ni}(\mathrm{nit})$ & Nitrato de $\mathrm{Ni}(\mathrm{II})$ & Agua \\
$\mathrm{Ni}(\mathrm{acac})$ & Acetilacetonato de Ni(II) & Acetona \\
\hline
\end{tabular}

Tabla II.3. Reactivos utilizados para la preparación de los sólidos impregnados con níquel. En la nomenclatura de los sólidos resultantes se añade al nombre indicado su contenido nominal en $\mathrm{NiO}$.

Para preparar las disoluciones de impregnación se disolvió la cantidad de sal necesaria en cada caso para la incorporación de la cantidad deseada de fase activa, teniendo en cuenta que en cada caso se partió de 6 gramos de arcilla pilareada. Al 
utilizar como precursores cloruro y nitrato de níquel, las disoluciones se prepararon utilizando agua bidestilada como disolvente; pero el acetilacetonato de níquel es insoluble en agua, por lo que en este caso se utilizó acetona como disolvente. En las impregnaciones realizadas con agua, las cantidades de fase activa fueron disueltas en la cantidad de disolvente necesaria para mojar el soporte, y los sólidos se obtuvieron en un único proceso de impregnación. En el caso del acetilacetonato de níquel, las cantidades necesarias de fase activa se disolvieron en la mínima cantidad posible de acetona, pero dada la baja solubilidad del acetilacetonato de níquel, el volumen de disolución fue en todos los casos mayor que la cantidad necesaria para mojar el soporte, por lo que fueron necesarios varios ciclos de impregnación para la incorporación de la totalidad de fase activa.

Los sólidos obtenidos tras cada proceso de impregnación se dejaron secar a temperatura ambiente durante 48 horas, tras lo que se calcinaron en atmósfera de aire para obtener los catalizadores metálicos soportados. Para realizar la calcinación, se elevó la temperatura con una rampa lenta de 1 o $\mathrm{C} / \mathrm{min}$ desde temperatura ambiente hasta $500{ }^{\circ} \mathrm{C}$, siempre en atmósfera de aire y manteniendo la temperatura de $500{ }^{\circ} \mathrm{C}$ durante dos horas. Igual que en el caso de los catalizadores soportados de cromo, al ser el soporte una arcilla pilareada, que ya ha sido previamente calcinada a $500{ }^{\circ} \mathrm{C}$, la rampa lenta de temperatura se utiliza en este caso para que los ligandos y contraiones de las sales utilizadas como precursoras se eliminen lentamente, evitando que su rápida descomposición provoque un colapso de la estructura laminar del soporte. La nomenclatura utilizada para los sólidos calcinados es la misma que la de los impregnados seguida de la temperatura de calcinación (Ej: $\mathrm{Ni}(\mathrm{acac}) 6 \rightarrow \mathrm{Ni}(\mathrm{acac}) 6-500)$.

\section{I.2.2. Método sol-gel}

La ruta para la preparación sol-gel se inicia normalmente con una disolución de sales metálicas inorgánicas o compuestos organometálicos; estos precursores son sometidos a una serie de reacciones de hidrólisis y polimerización para formar una suspensión coloidal o sol que mediante hidrólisis y condensación forma un material sólido lleno de disolvente ( $\mathrm{gel}$ ). Durante el periodo de envejecimiento el gel expulsa el solvente y agua residual. Utilizando este método, se ha intentado preparar una saponita sintética utilizando como precursores compuestos de $\mathrm{Si}^{4+}, \mathrm{Al}^{3+}, \mathrm{Na}^{+}$y $\mathrm{Ni}^{2+}$. 
La capacidad catalítica del níquel, así como su facilidad para ocupar posiciones octaédricas en los minerales arcillosos, son las propiedades por las que se elige este elemento como precursor.

Experimentalmente, el proceso se inició mediante la disolución de las cantidades necesarias de cloruro de níquel, carbonato sódico y cloruro de aluminio en diclorometano, en un matraz de dos bocas. A continuación, se añadió tetraetilortosilicato (TEOS) y éter isopropílico. La mezcla resultante, con $\mathrm{pH}=2,0$, se mantuvo a reflujo a $110{ }^{\circ} \mathrm{C}$, con agitación y en atmósfera de argón, durante 4 horas. Una vez finalizado este tiempo, la mezcla reaccionante se dejó enfriar y se envejeció durante una noche a temperatura ambiente. El exceso de disolvente se eliminó mediante rotavapor $y$ el sólido fue lavado sucesivamente con diclorometano, acetonitrilo y metanol (utilizando los disolventes en este orden). Tras el lavado, el sólido se secó a $70^{\circ} \mathrm{C}$ durante 48 horas, y la muestra resultante se denominó SG-seca (SG denota el procedimiento de obtención del sólido).

Los catalizadores fueron obtenidos por tratamientos térmicos a diferentes temperaturas, en concreto $300,450,600$ y 1000 º . La velocidad de calentamiento fue en todos los casos de $30{ }^{\circ} \mathrm{C} / \mathrm{min}$, manteniendo la temperatura final de calcinación durante 4 horas. Tras las calcinaciones, los sólidos se dejaron enfriar a temperatura ambiente, obteniéndose así los catalizadores finales que se denominan SG-300, SG-450, SG-600 y SG-1000, respectivamente, en función de la temperatura a la que han sido calcinados (Tabla II.4).

\begin{tabular}{|ccc|}
\hline Nomenclatura & Reactivos & $\begin{array}{c}\text { Temperatura de secado } \\
\text { / calcinación / }{ }^{\circ} \mathrm{C}\end{array}$ \\
\hline SG-Blanco & $\mathrm{Na}_{2} \mathrm{CO}_{3}+\mathrm{AlCl}_{3}+\mathrm{TEOS}$ & 70 \\
SG-300 & $\mathrm{NiCl}_{2}+\mathrm{Na}_{2} \mathrm{CO}_{3}+\mathrm{AlCl}_{3}+\mathrm{TEOS}$ & 300 \\
SG-450 & $\mathrm{NiCl}_{2}+\mathrm{Na}_{2} \mathrm{CO}_{3}+\mathrm{AlCl}_{3}+\mathrm{TEOS}$ & 450 \\
SG-600 & $\mathrm{NiCl}_{2}+\mathrm{Na}_{2} \mathrm{CO}_{3}+\mathrm{AlCl}_{3}+$ TEOS & 600 \\
SG-1000 & $\mathrm{NiCl}_{2}+\mathrm{Na}_{2} \mathrm{CO}_{3}+\mathrm{AlCl}_{3}+$ TEOS & 1000 \\
\hline
\end{tabular}

Tabla II.4. Reactivos utilizados para la preparación de los sólidos por el método sol-gel, y nomenclatura de los sólidos resultantes.

Además de los sólidos mencionados, se preparó con fines comparativos una muestra en ausencia de níquel, para analizar la influencia de este elemento en el 
comportamiento catalítico. Para ello, se trataron los precursores de $\mathrm{Si}^{4+}, \mathrm{Al}^{3+}$ y $\mathrm{Na}^{+}$ por el procedimiento antes descrito, manteniendo todas las variables del proceso. La muestra seca así obtenida fue denominada SG-blanco.

\section{I.2.3. Método de co-precipitación}

La preparación de los sólidos mediante este método comenzó con la disolución en un matraz de dos bocas de cloruro de níquel, carbonato sódico y cloruro de aluminio en diclorometano. A continuación, de añadieron TEOS y amoníaco. Tras esta adición, se observó una gelificación de la mezcla, y se añadieron $40 \mathrm{~mL}$ de agua y $9 \mathrm{~mL}$ de amoníaco. La mezcla resultante, con $\mathrm{pH}=11,0$, se mantuvo a reflujo a $60{ }^{\circ} \mathrm{C}$ y con agitación magnética durante 20 horas. Al igual que en la preparación por el método sol-gel, el sólido resultante se sometió a lavados sucesivos utilizando diclorometano, acetonitrilo y metanol como disolventes. Tras el lavado, el sólido se secó a $70{ }^{\circ} \mathrm{C}$ durante 48 horas, y el sólido así obtenido fue denominado CP-seco (como en el caso anterior, CP denota el método de obtención).

Para obtener los catalizadores finales, las muestras se sometieron a tratamiento térmico a 300, 450, 600 y $1000{ }^{\circ} \mathrm{C}$, utilizando rampas de temperatura de 30 $\mathrm{C} / \mathrm{min}$ y manteniendo la temperatura de calcinación durante 4 horas. Como en el caso anterior, se preparó una muestra comparativa en ausencia de níquel, obtenida manteniendo todas las condiciones de la reacción, pero sin añadir el precursor de este elemento. La muestra así obtenida se denominó CP-blanco.

\section{II.3. Métodos experimentales}

\section{I.3.1. Análisis químico}

El análisis químico de las arcillas de partida y de los sólidos preparados por los distintos métodos fue realizado por los laboratorios Activation Laboratories Ltd., en Ancaster, Ontario, Canadá. La técnica utilizada para el análisis fue la Espectrometría de Absorción de Plasma Inductivamente Acoplado (ICPS). Para llevar a cabo el proceso, las muestras se mezclan previamente con metaborato y 
tetraborato de litio y se funden en un horno de inducción. La mezcla fundida se introduce en una disolución que contiene un 5\% de ácido nítrico hasta su total disolución. Las composiciones obtenidas son garantizadas por el análisis simultáneo de varios patrones habitualmente usados por dicho laboratorio.

\section{II.3.2. Difracción de rayos $X$}

Los estudios de difracción de rayos $\mathrm{X}$ fueron realizados en un difractómetro Siemens D500 provisto de un sistema de control Difract-AT y como almacenador de datos un microprocesador DACO-MP. El equipo utiliza un tubo de cobre para la obtención de la radiación, y un filtro de níquel, así como un sistema de rendijas consecutivas, para su colimación, por lo que los análisis se realizan empleando radiación Cu Ka filtrada $(\lambda=1,5418 \AA)$. Las condiciones de trabajo fueron $30 \mathrm{~mA}$ de intensidad, $40 \mathrm{kV}$ de voltaje y velocidad de barrido de $2^{\circ}$ de ángulos de Bragg (2 $\theta$ ) por minuto. El análisis de todos los sólidos se realizó entre 2 y $70^{\circ}$ de $2 \theta$ sobre muestras en polvo no orientado.

La difracción de rayos $X$ es una técnica ampliamente empleada en el estudio de compuestos laminares. En este trabajo se utiliza para caracterizar las distintas muestras, estudiando las reflexiones basales que permiten determinar si ha habido o no una correcta intercalación en la arcilla de partida. Además permite analizar la posible presencia de otras fases, tales como óxidos e hidróxidos metálicos cristalinos, en los sólidos pilareados y en los impregnados, así como identificar las distintas fases obtenidas en las preparaciones realizadas por los métodos sol-gel y de co-precipitación. Para ello, los difractogramas obtenidos fueron analizados utilizando el programa EVA (Graphics EVAluation Program) y, en los casos necesarios, comparados con los patrones del fichero Joint Committee on Powder Diffraction Standards, JCPDS [6]. Estas comparaciones permitieron reconocer cualitativamente las fases presentes en cada una de las muestras, y en el caso de la muestra natural, evaluar su composición mineralógica por la aplicación del método de los poderes reflectantes [7]. 


\section{I.3.3. Análisis térmicos}

\section{II.3.3.a. Análisis térmico gravimétrico}

Los análisis térmicos gravimétricos (ATG) fueron realizados en una termobalanza Perkin-Elmer TGA-7 con estación de datos TAC7/DX y programa de análisis TGA7 HT. Los análisis se llevaron a cabo mediante una rampa de temperatura de $10^{\circ} \mathrm{C} / \mathrm{min}$ hasta $1000{ }^{\circ} \mathrm{C}$. Para ello, se necesitan unos $20 \mathrm{mg}$ de muestra y se hace pasar un flujo continuo de $\mathrm{O}_{2} \circ \mathrm{N}_{2}$ de $35 \mathrm{~cm}^{3} / \mathrm{min}$ en función de la atmósfera en la que se desee estudiar el proceso térmico.

\section{I.3.3.b. Análisis térmico diferencial}

Para los estudios de análisis térmico diferencial (DTA) se empleó un equipo Perkin-Elmer DTA-7 con estación de datos TAC7/DX y programa de análisis DTA7, dotado de un horno vertical, con termopares y registro automático. Al igual que en el análisis térmico gravimétrico, se realizó una rampa de calentamiento de $10{ }^{\circ} \mathrm{C} / \mathrm{min}$ hasta alcanzar los $1000{ }^{\circ} \mathrm{C}$. En este caso, también se mantienen atmósferas dinámicas de oxígeno o nitrógeno. La referencia utilizada fue una alúmina de Perkin-Elmer, especial para análisis térmico.

\section{II.3.3.c. Reducción a temperatura programada}

La reducción a temperatura programada (TPR) se realizó en un equipo Micromeritics TPR/TPD 2900, entre temperatura ambiente y $800{ }^{\circ} \mathrm{C}$, utilizando una rampa de calentamiento de $10{ }^{\circ} \mathrm{C} / \mathrm{min}$, utilizando una corriente de gas que contiene hidrógeno en argón al $5 \%$ en volumen ( $60 \mathrm{~mL} / \mathrm{min})$. El consumo de hidrógeno se mide mediante un detector de conductividad térmica. El sistema cuenta además con una trampa de isopropanol para eliminar el agua, el amoníaco y otros posibles compuestos que puedan formarse durante el proceso de reducción y afectar a la medida. 


\section{I.3.4. Espectroscopia Visible-Ultravioleta por Reflectancia Difusa}

Los espectros electrónicos de las muestras se registraron en el intervalo 1100$200 \mathrm{~nm}$ mediante reflectancia difusa (RD-UV-Vis) en un espectrofotómetro PerkinElmer Lambda 35 con dispositivo de esfera integradora incorporado Labsphere RSAPE-20, y acoplado a un PC con software UV WinLab 2.85, utilizando una rendija de $2 \mathrm{~nm}$ y $\mathrm{MgO}$ como referencia.

\section{II.3.5. Espectroscopia infrarroja por transformada de Fourier}

Los espectros de infrarrojo (FT-IR) fueron obtenidos con un espectrómetro Perkin-Elmer 1730 Infrared Fourier Transform Spectrometer, provisto de una fuente de radiación láser He-Ne $(\lambda=632,8 \mathrm{~nm})$. Los datos se recogen y se tratan con el programa Spectrum for Windows. Se realizan los espectros de las muestras sobre bromuro potásico de uso espectroscópico. Esta técnica consiste en hacer una gran dilución de la muestra en bromuro potásico, 1/300 aproximadamente, utilizando una pequeña cantidad de muestra ( $1 \mathrm{mg}$, aproximadamente). $\mathrm{El} \mathrm{KBr}$ debe estar previamente molido y seco en estufa a $110^{\circ} \mathrm{C}$. Una vez mezclado con la muestra, se obtiene una pastilla por prensado a $8 \mathrm{~T} / \mathrm{cm}^{2}$ y se registra el espectro entre 4000 y $400 \mathrm{~cm}^{-1}$, efectuando 15 barridos para cada muestra.

\section{I.3.6. I sotermas de adsorción-desorción de nitrógeno a 77K}

Los estudios de área superficial y porosidad de las muestras se realizaron a partir de las isotermas de adsorción-desorción de nitrógeno a $77 \mathrm{~K}\left(-196^{\circ} \mathrm{C}\right)$. Las isotermas se registraron en un equipo volumétrico automático Micromeritics ASAP 2010. Antes de realizar el estudio superficial, las muestras fueron pretratadas en el propio equipo. Con objeto de limpiar la superficie de los sólidos, se mantienen a vacío durante dos horas a temperatura ambiente, hasta que la presión es constante e inferior a $50 \mu \mathrm{m}$ de $\mathrm{Hg}$, tras lo cual se aumenta la temperatura hasta $110{ }^{\circ} \mathrm{C}$, hasta volver a alcanzar dicha presión, o durante un tiempo mínimo de dos horas.

El análisis se realiza sobre unos $100 \mathrm{mg}$ de muestra, tomando medidas a diferentes presiones y permitiendo al sistema alcanzar el equilibrio en cada uno de los pasos. La isoterma completa se divide en dos ramas, la de adsorción y la de 
desorción. El análisis de la rama de adsorción se realiza en dos etapas sucesivas. En la primera de ellas, se inyectan dosis constantes de $4 \mathrm{~cm}^{3} \mathrm{~N}_{2}$ (CNPT) dejando un tiempo de estabilización entre 1 y 1,5 horas; este proceso se mantiene hasta alcanzar presiones superiores a $p / p^{0}=0,1$. Una vez alcanzado este valor, se hacen medidas de presiones relativas según una tabla de valores previamente fijada, hasta completar el número de datos deseado, tanto en la rama de adsorción como en la de desorción.

Mediante el estudio de los datos obtenidos, se calcula el área por aplicación del método BET [8] y de Langmuir [9], utilizando valores de presiones relativas entre 0,10 y 0,35 . También se estudia la distribución del tamaño de poros aplicando el método de Horvath-Kawazoe [10]. Así mismo, se emplea el método de la recta $\mathrm{t}$ para establecer la posible existencia de microporos [11].

\section{3.7. Microscopía electrónica}

\section{I.3.7.a. Microscopía electrónica de transmisión}

Los estudios de microscopía electrónica de transmisión (TEM) fueron realizados en el Servicio de Microscopía Electrónica de la Universidad de Salamanca, utilizando un microscopio ZEISS-902. Antes de realizar el estudio microscópico, las muestras se someten a un pretratamiento, dispersando una pequeña cantidad de sólido en un baño de ultrasonidos, y a continuación se deposita una gota de esta suspensión sobre una rendija de cobre previamente impregnada, mediante arco voltaico, con una película de carbono amorfo.

\section{I.3.7.b. Microscopia electrónica de barrido}

La microscopía electrónica de barrido (SEM) se realizó en el mismo Servicio utilizando un equipo Microscope Zeiss DSM 940, conectado a un Scan Converter DSC-1024 G Sony, gracias al cual las imágenes pueden ser digitalizadas. El pretratamiento de estas muestras consiste en cubrirlas con una fina película de oro. Para ello, se introducen en una cámara donde se someten a alto vacío, y posteriormente se introduce una pequeña cantidad de argón y se aplica un voltaje de $1500 \mathrm{~V}$ durante $60 \mathrm{~s}$. Este voltaje produce la ionización del argón, produciendo 
cationes $\mathrm{Ar}^{+}$que chocan contra un cátodo de oro situado sobre las muestras. Las colisiones de los iones de gas contra el metal producen el desprendimiento de átomos de oro que cubren la muestra. De esta manera, se convierten en muestras conductoras, condición necesaria para su posterior bombardeo con electrones en el microscopio.

\section{II.4. Evaluación de la actividad catalítica}

\section{II.4.1. Reacción DeNOx}

La actividad de los diferentes catalizadores en la reducción de óxidos de nitrógeno fue estudiada en un reactor de flujo, alimentado por gases de contenido conocido, y en el que los gases de salida se conectan en línea con un espectrómetro infrarrojo Perkin-Elmer 1725X FTIR, con una celda de transmisión de múltiples reflexiones Infrared Analysis Inc.

Se utilizó propeno como gas reductor y argón como gas portador. La alimentación del reactor contenía cantidades estequiométricas de oxidante y reductor, produciéndose la siguiente reacción ideal:

$\mathrm{C}_{3} \mathrm{H}_{6}+9 \mathrm{NO} \rightarrow 3 \mathrm{CO}_{2}+3 \mathrm{H}_{2} \mathrm{O}+9 / 2 \mathrm{~N}_{2}$

Se utilizó un flujo total de entrada al reactor de $200 \mathrm{~mL} / \mathrm{min}$, con un contenido de $0,1 \%$ de reductor y $0,9 \%$ de oxidante, además del gas portador. La cantidad de catalizador introducida en el reactor fue de 0,63 gramos de sólido tamizado a un tamaño entre 0,125 y $0,250 \mathrm{~mm}$, con lo que se obtiene finalmente una velocidad espacial de $19028 \mathrm{~h}^{-1}$.

Las medidas de reactividad se realizan en estado estacionario en intervalos de $50{ }^{\circ} \mathrm{C}$, desde 200 hasta $500{ }^{\circ} \mathrm{C}$. Cada uno de los calentamientos se realizó en rampas de 5 o $\mathrm{C} / \mathrm{min}$, manteniendo la temperatura constante en cada etapa el tiempo necesario para que la reacción sea medida en estado estacionario. Una vez estabilizada la reacción, se realiza el espectro de infrarrojo del gas eluyente del reactor. El esquema del equipo completo se recoge en la Figura II.4. 


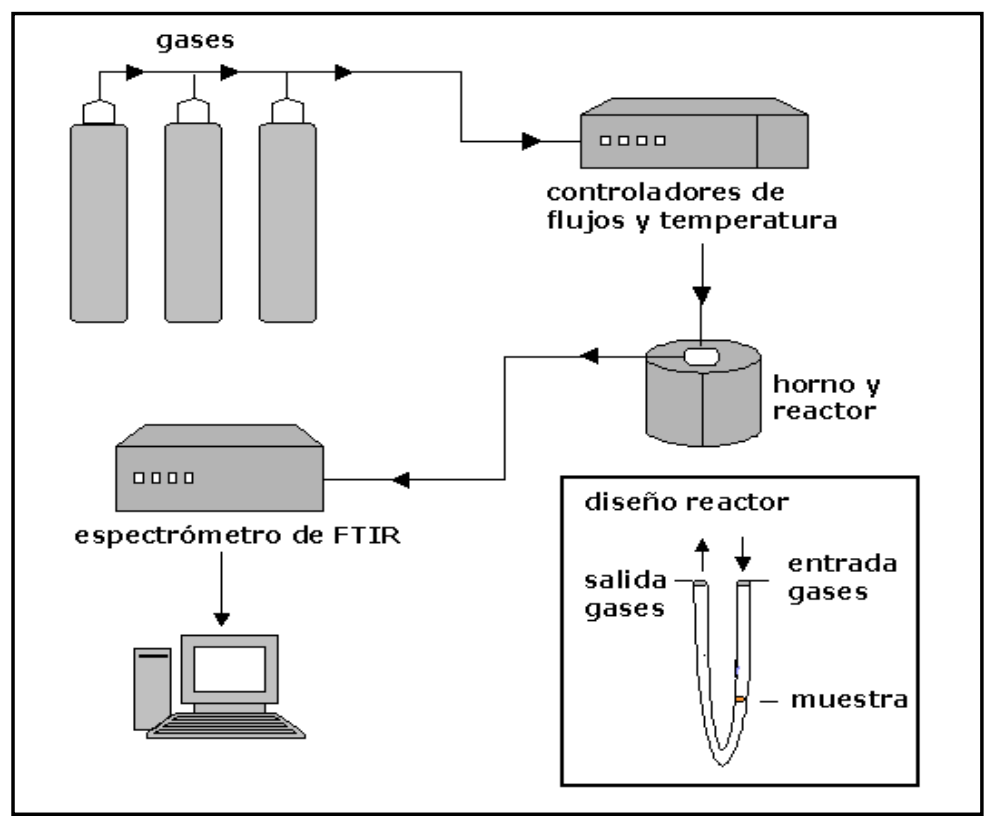

Figura II.4. Esquema del sistema de reacción en el que se realizan las medidas de actividad catalítica en reacciones DeNOx.

Antes de llevar a cabo las pruebas para determinar el comportamiento catalítico de los sólidos se deben analizar las variables del reactor que pueden influir en las medidas que se van a realizar. Cada uno de los componentes del reactor debe calibrarse, y se debe trabajar con un flujo, velocidad espacial, tamaño de partícula, etc. adecuados para obtener los mejores resultados.

\section{II.4.1.a. Rectas de calibrado de reactivos y productos}

Como se ha indicado, las medidas se llevan a cabo con un espectrómetro infrarrojo, por lo que se deben identificar las bandas de cada uno de los reactivos y productos, tanto de los esperados como de posibles productos de combustiones incompletas o de reacciones secundarias, y comprobar las interferencias entre ellas. En primer lugar, se determinan las bandas que pueden ser utilizadas como diagnóstico para cada reactivo o producto, y a continuación determinamos, para cada una de ellas, si es más adecuado utilizar su intensidad o su área para realizar el calibrado de las concentraciones. Con diferentes concentraciones de entrada, se hace un calibrado en todo el intervalo de trabajo. En la siguiente Tabla se indican las especies estudiadas, y la magnitud empleada para su análisis, elegida tras 
analizar su banda diagnóstico así como las posibles interferencias con otras bandas que aparecen en zonas próximas.

\begin{tabular}{|c|c|c|}
\hline Especie analizada & Banda $/ \mathrm{cm}^{-1}$ & Magnitud \\
\hline $\mathrm{CO}$ & 2118 & Intensidad \\
$\mathrm{CO}_{2}$ & 670 & Intensidad \\
$\mathrm{NO}_{2}$ & 1631 & Intensidad \\
$\mathrm{N}_{2} \mathrm{O}$ & 1300 & Intensidad \\
$\mathrm{NO}$ & $1960-1880$ & Área \\
$\mathrm{C}_{3} \mathrm{H}_{6}$ & $1100-800$ & Área \\
\hline
\end{tabular}

Tabla II.5. Especies analizadas, posición de las bandas diagnóstico y magnitudes utilizadas para el análisis de los espectros FTIR en el reactor catalítico.

\section{I.4.1.b. Estudio del tamaño de partícula}

El tamaño de las partículas de catalizador introducidas en el sistema es una variable importante; tamaños demasiado pequeños originan problemas de difusión, produciendo altas presiones de los gases en el interior del reactor, o incluso un taponamiento en el mismo. Por otro lado, los tamaños demasiado grandes dan lugar a conversiones bajas ya que disponen de menos centros activos accesibles para llevar a cabo la reacción. El objetivo es encontrar un tamaño de tamizado adecuado situado en un compromiso entre ambos, con la máxima conversión posible y que no origine problemas de difusión de los gases.

\section{I.4.1.c. Cálculo de la velocidad espacial}

La velocidad espacial de la reacción viene dada por la ecuación:

$$
V=(F \rho) / m
$$

en la que $\mathrm{F}$ representa el flujo total de gases que entran en el reactor, $\mathrm{m}$ la masa de catalizador que se introduce en el reactor, $\rho$ su densidad, y $V$ la velocidad espacial. Las diferentes cantidades de catalizador que se pueden introducir en el sistema pueden dar lugar a conversiones muy diferentes. El estudio de la velocidad espacial se lleva a cabo introduciendo diferentes cantidades de catalizador y 
estudiando las conversiones. Como cantidad de partida se suele usar una semejante a las utilizadas en ensayos catalíticos anteriores, o a las descritas en bibliografía, para después utilizar cantidades superiores e inferiores a ésta hasta encontrar un valor óptimo. En nuestro caso, se utilizó una velocidad espacial de $19028 \mathrm{~h}^{-1}$, utilizada con anterioridad en el mismo reactor para catalizadores Fearcilla comparables a los ahora estudiados [12-13].

\section{I.4.1.d. Tratamiento matemático de los resultados obtenidos}

Los espectros obtenidos se analizan mediante un programa matemático, que calcula las concentraciones de cada uno de los gases a la salida del reactor. De las cantidades deducidas de los espectros infrarrojos se calculan las conversiones de cada una de las especies, que indican el porcentaje de compuesto que reacciona durante el proceso catalítico. La conversión de cada compuesto se realiza de la siguiente manera:

- Conversión de $\mathrm{NO}_{\mathrm{x}}$ : Indica el porcentaje de óxidos de nitrógeno que reaccionan en el proceso catalítico:

$$
\mathrm{NO}_{\mathrm{x} \text { conv }}=\left(1-\frac{\mathrm{NO}+N \mathrm{O}_{2}}{N O_{x i}}\right) \times 100
$$

Donde $\mathrm{NO}_{\mathrm{x}}$ conv es la cantidad de $\mathrm{NO}_{\mathrm{x}}$ convertida en el transcurso de la reacción y $\mathrm{NO}_{\mathrm{xi}}$ es la cantidad inicial de $\mathrm{NO}_{\mathrm{x}}$, cuyo valor es: $\mathrm{NO}_{\mathrm{xi}}=\mathrm{NO}$ $+\mathrm{NO}_{2}+\left(2 \times \mathrm{N}_{2} \mathrm{O}\right)$.

- Conversión de hidrocarburo: Indica el porcentaje de propeno que se consume durante la reacción:

$$
\mathrm{HC}_{\text {conv }}=\left(1-\frac{H C}{H C i}\right) \times 100
$$

$\mathrm{HC}_{\text {conv }}$ es la cantidad de hidrocarburo convertida, $\mathrm{HC}$ la medida en cada espectro y $\mathrm{HC}_{i}$ es la cantidad inicial que entra en el reactor.

- Producción de $\mathrm{CO}$ : Indica la cantidad de $\mathrm{CO}\left(\mathrm{CO}_{\text {prod }}\right)$ formada en la combustión incompleta de hidrocarburo, expresada en función del CO 
analizado en cada momento, referido a la cantidad de propeno inicialmente introducido, considerando que una molécula de hidrocarburo puede producir tres moléculas de CO.

$$
\mathrm{CO}_{\text {prod }}=\left(\frac{C O}{3 \times H C_{i}}\right) \times 100
$$

- Rendimiento a $\mathrm{N}_{2}$ : Indica el porcentaje de $\mathrm{NO}_{x}$ que se ha transformado en nitrógeno molecular durante la reacción ( $\left.\mathrm{N}_{2 \text { rend }}\right)$. Se calcula mediante un balance de masas ya que este compuesto no es activo en infrarrojo. Dado que en ningún caso se detectó ningún otro compuesto nitrogenado, el balance de masas realizado es el siguiente:

$$
\mathrm{N}_{2 \text { rend }}=\left(1-\frac{N O_{X}}{N O_{X i}}\right) \times 100
$$

\section{II .4.2. Reacciones de oxidación de Z-cicloocteno y ciclohexano}

La actividad catalítica de las arcillas impregnadas con níquel se estudió en dos reacciones de oxidación, la epoxidación de (Z)-cicloocteno y la oxidación de ciclohexano. Estas reacciones se encuentran esquematizadas en la Figura II.5.
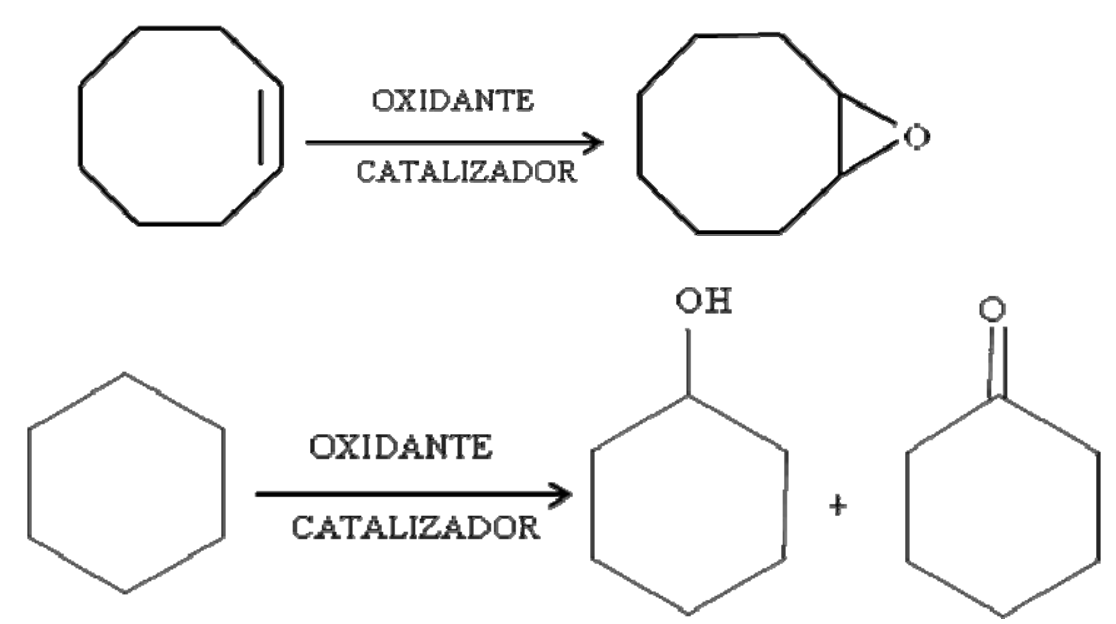

Figura II.5. Reacciones de oxidación de (Z)-cicloocteno y de ciclohexano.

En ambos casos se utilizaron dos oxidantes, el yodolsilbenceno y el peróxido de hidrógeno. El segundo de ellos no es contaminante, ya que el único subproducto que genera es agua, por lo que es el oxidante ideal desde el punto de vista 
industrial, mientras que el primero de ellos se ha utilizado por la información que permite obtener sobre el desarrollo de la reacción. El yodosilbenceno (PhIO) se sintetiza mediante hidrólisis de diacetato de yodosilbenceno, según la metodología descrita por Saltzman y Sharefkin [14], de acuerdo a la siguiente reacción:

$$
\mathrm{C}_{6} \mathrm{H}_{5} \mathrm{I}\left(\mathrm{OCOCH}_{3}\right)_{2}+2 \mathrm{NaOH} \rightarrow \mathrm{C}_{6} \mathrm{H}_{5} \mathrm{IO}+2 \mathrm{NaOCOCH}_{3}+\mathrm{H}_{2} \mathrm{O}
$$

El producto de la hidrólisis se seca a presión reducida y se conserva a $5{ }^{\circ} \mathrm{C}$. Su pureza se controla periódicamente mediante valoración yodométrica. En medio ácido, el yodosilbenceno reacciona con yoduro liberando yodo, mediante la reacción:

$$
\mathrm{C}_{6} \mathrm{H}_{5} \mathrm{IO}+2 \mathrm{I}^{-} \rightarrow \mathrm{C}_{6} \mathrm{H}_{5} \mathrm{I}+\mathrm{H}_{2} \mathrm{O}+\mathrm{I}_{2}
$$

El yodo generado es posteriormente valorado con tiosulfato.

Para llevar a cabo la oxidación con yodosilbenceno, se introducen $10 \mathrm{mg}$ del catalizador a estudiar en un vial de $4 \mathrm{~cm}^{3}$ sellado mediante un septo de silicona y revestido de teflón; a continuación se añaden $22,7 \mathrm{mmol}$ de PhIO en una mezcla de 1,2 dicloroetano y 1,15 mmoles de (Z)-cicloocteno (previamente purificado mediante una columna de alúmina) o 1,39 mmoles de ciclohexano, y 0,01 $\mathrm{cm}^{3} \mathrm{de}$ ciclohexanona o n-octanol, respectivamente, como patrones internos de las dos reacciones consideradas.

En el caso de la oxidación con peróxido de hidrógeno, las reacciones se llevan a cabo exactamente de la misma manera, con la única diferencia de añadir $25 \mu \mathrm{L}$ de peróxido de hidrógeno al 70\% como oxidante en sustitución de yodosilbenceno. Todas las reacciones se realizan a $25^{\circ} \mathrm{C}$ de temperatura y presión atmosférica.

Una vez concluida cada reacción, la disolución reaccionante se separa del catalizador mediante centrifugación, el catalizador se lava repetidas veces con 1,2dicloroetano y los productos de reacción se analizan mediante cromatografía de gases (Hewlett-Packard 6890). Los rendimientos se calculan por comparación con curvas de calibrado obtenidas a partir de compuestos puros. Para tener un mejor conocimiento de las reacciones, éstas se llevan a cabo utilizando pruebas "en 
blanco", en concreto utilizando la arcilla natural y la arcilla pilareada, sin contener níquel, o sin añadir oxidante, y siguiendo el mismo procedimiento ya descrito.

\section{I.5. Reactivos utilizados}

La síntesis de los policationes de intercalación se llevó a cabo con los siguientes reactivos:

- Cloruro de aluminio hexahidratado, $\mathrm{AlCl}_{3} \cdot 6 \mathrm{H}_{2} \mathrm{O}$, Panreac, purísimo.

- Cloruro de cromo(III) hexahidratado, $\mathrm{CrCl}_{3} \cdot 6 \mathrm{H}_{2} \mathrm{O}$, Aldrich, purísimo.

- Hidróxido sódico, $\mathrm{NaOH}$, Panreac, 97\%, lentejas.

Los análisis térmicos y las isotermas de adsorción y desorción de nitrógeno se realizaron con los siguientes gases:

- Aire sintético $\left(\mathrm{N}_{2}+\mathrm{O}_{2}\right), \mathrm{N}-49$, Sociedad Española de Oxígeno.

- Nitrógeno, $\mathrm{N}_{2}$, Alphagaz, N-50, Sociedad Española de Oxígeno.

- Helio, He, Alphagaz, N-48, Sociedad Española de Oxígeno.

- Oxígeno, $\mathrm{O}_{2}$, Alphagaz, N-50, Sociedad Española de Oxígeno.

- Hidrógeno al 5\% diluido en argón (Air Liquide).

Las pastillas para la obtención de los espectros de infrarrojo fueron preparadas utilizando:

- Bromuro potásico, $\mathrm{KBr}$, Panreac, para espectroscopia IR.

Para la síntesis de catalizadores soportados basados en cromo, se utilizaron como precursores los siguientes compuestos:

- Acetato de cromo(II) monohidratado dímero, $\left[\mathrm{Cr}\left(\mathrm{CH}_{3} \mathrm{CO}_{2}\right)_{2} \mathrm{H}_{2} \mathrm{O}\right]_{2}$, Aldrich, $98 \%$.

- Acetilacetonato de cromo (III), $\mathrm{Cr}\left(\mathrm{C}_{5} \mathrm{H}_{8} \mathrm{O}_{2}\right)_{3}$, Aldrich, 97\%.

- Cromato amónico, $\left(\mathrm{NH}_{4}\right)_{2} \mathrm{CrO}_{4}$, Aldrich, $98 \%$.

- Nitrato de cromo(III) nonahidratado, $\mathrm{Cr}\left(\mathrm{NO}_{3}\right)_{3} \cdot 9 \mathrm{H}_{2} \mathrm{O}, 98 \%$.

- Acetona, $\mathrm{CH}_{3} \mathrm{COCH}_{3}$, Panreac, purísimo.

- Alúmina esferalita 505, $\mathrm{Al}_{2} \mathrm{O}_{3}$, Procatalyse, 100-200 $\mu \mathrm{m}$. 
La actividad catalítica en la reacción DeNOx de los catalizadores preparados fue medida utilizando los siguientes gases:

- Propeno, $\mathrm{C}_{3} \mathrm{H}_{6}, 1,86 \%$ diluido en argón, Sociedad Española de Oxígeno.

- Óxido de nitrógeno, $\mathrm{NO}, \mathrm{C}-20\left(98 \% \mathrm{NO}+1 \% \mathrm{NO}_{2}+0,5 \% \mathrm{~N}_{2} \mathrm{O}\right)$, Carburos Metálicos.

- Argón, Ar, Alphagaz, Sociedad Española de Oxígeno.

- Aire sintético, $\left(\mathrm{N}_{2}+\mathrm{O}_{2}\right)$, Sociedad Española de Oxígeno.

Para la preparación de los catalizadores de níquel se utilizaron los siguientes reactivos:

- Nitrato de níquel hexahidratado, $\mathrm{Ni}\left(\mathrm{NO}_{3}\right)_{2} \cdot 6 \mathrm{H}_{2} \mathrm{O}$, Aldrich, 99,999\%.

- Acetilacetonato de níquel, $\mathrm{Ni}\left(\mathrm{C}_{5} \mathrm{H}_{8} \mathrm{O}_{2}\right)_{2}$, Aldrich, 95\%.

- Cloruro de níquel hexahidratado, $\mathrm{NiCl}_{2} \cdot 6 \mathrm{H}_{2} \mathrm{O}$, Aldrich, 99,95\%.

- Acetona, $\mathrm{CH}_{3} \mathrm{COCH}_{3}$, Panreac, purísimo.

Para la preparación de las muestras mediante los métodos sol-gel y coprecipitación se utilizaron lo siguientes reactivos:

- Tetraetilortosilicato, $\mathrm{Si}\left(\mathrm{OC}_{2} \mathrm{H}_{5}\right)_{4}$, TEOS, Sigma-Aldrich, 99,999.

- Cloruro de aluminio, $\mathrm{AlCl}_{3}$, Merck, 99,999\%.

- Cloruro de níquel hexahidratado, $\mathrm{NiCl}_{2} \cdot 6 \mathrm{H}_{2} \mathrm{O}$, Merck, $97 \%$.

- Carbonato sódico, $\mathrm{Na}_{2} \mathrm{CO}_{3}$, Merck, 99\%.

- Diclorometano, $\mathrm{CH}_{2} \mathrm{Cl}_{2}$, Aldrich, 99,8\%.

- Isopropil éter anhidro, $\left(\mathrm{CH}_{3}\right)_{2} \mathrm{CHOCH}\left(\mathrm{CH}_{3}\right)_{2}$, Aldrich, 99,0.

- Acetonitrilo, $\mathrm{CH}_{3} \mathrm{CN}$, Merck, 99\%.

- Metanol, $\mathrm{HCH}_{2} \mathrm{OH}$, Merck, 99,9\%.

- Amoníaco, $\mathrm{NH}_{3}$, Fluka, 97\%.

Para la evaluación de la actividad catalítica en las reacciones de oxidación de (Z)-cicloocteno y ciclohexano se utilizaron los siguientes reactivos:

- Peróxido de hidrogeno, $\mathrm{H}_{2} \mathrm{O}_{2}$, Peróxidos do Brasil - Solvay, $70 \%$ v/v.

- Diacetato de yodosilbenceno, $\mathrm{C}_{6} \mathrm{H}_{5} \mathrm{I}\left(\mathrm{OCOCH}_{3}\right)_{2}$, Aldrich, $98 \%$. 
- Diclorometano, $\mathrm{CH}_{2} \mathrm{Cl}_{2}$, Aldrich, 99,8\%.

- 1,2-Dicloroetano, $\mathrm{CH}_{2} \mathrm{Cl}-\mathrm{CH}_{2} \mathrm{Cl}$, Merck, 99\%

- (Z)-cicloocteno, Acros Organics, 95\%.

- Ciclohexano, Acros Organics, 99\%.

- Ciclohexanol, Acros Organics, 99\%.

- Ciclohexanona, Acros Organics, 99\%.

- n-Octanol, Acros Organics, 99\%.

\section{I l.6. Bibliografía}

[1] Vaughan D.E.W. Pillared clays-A historical perspective. Catalysis Today 2, 187198 (1988).

[2] Jones S.L. The preparation and solution chemistry of $\mathrm{Al}(\mathrm{III})$ and $\mathrm{Zr}$ (IV) pillaring species. Catalysis Today 2, 209-217 (1988).

[3] Gil A., Gandía L.M., Vicente M.A. Recent advances in the synthesis and catalytic applications of pillared clays. Catalysis Reviews-Science and Engineering 42, 145-212 (2000).

[4] Brindley G.W., Yamakana S. A study of hydroxychromium montmorillonites and the form of the hydroxy-chromium polymers. American Mineralogist 64, 830835 (1979).

[5] Burriel Martí F., Lucena Conde F., Arribas Jimeno S., Hernández Méndez J. Química analítica cualitativa. Editorial Paraninfo. Madrid (1992).

[6] Joint Committee on Powder Diffraction Standards. JCPDS File. International Centre for Diffraction Data. Philadelphia (1977).

[7] Martín Pozas J.M. Análisis cuantitativo de fases cristalinas por DRX. En: Difracción de muestras policristalinas. Método de Debye-Scherrer. Instituto de Ciencias de la Educación de la Universidad de Valladolid (1975).

[8] Brunauer S., Emmett P.H., Teller E. Adsorption of gases in multimolecular layers. Journal of the American Chemical Society 20, 1553-1564 (1937). 
[9] Langmuir I. The adsorption of gases on plane surfaces of glass, mica and platinum. Journal of the American Chemical Society 40, 1361-1403 (1918).

[10] Horvath G., Kawazoe K. Method for the calculation of effective pore size distribution in molecular sieve carbon. Journal of Chemical Engineering of Japan $16,470-475$ (1983).

[11] Lippens B.C., de Boer J.H. Studies on pore systems in catalysis. V. The $t$ method. Journal of Catalysis 4, 319-323 (1965).

[12] Belver C., Vicente M.A., Martínez-Arias A., Fernández-García M. Fe-Saponite Pillared and Impregnated Catalysts. II. Nature of the Iron Species Active for the Reduction of NOx with Propene. Applied Catalysis B: Environmental 50, 227-234 (2004).

[13] Belver C., Vicente M.A., Fernández-García M., Martínez-Arias A. Supported catalysts for DeNOx reaction based on iron clays. Journal of Molecular Catalysis A: Chemical 219, 309-313 (2004).

[14] Saltzman H., Sharefkin J.G. Iodosobenzene. Organic Syntheses, Collective Volume 5, 658 (1973); Vol. 43, 60 (1963). 


\section{Capítulo I I I}

Chromium-saponite clay catalysts: Preparation, characterization and catalytic performance in propene oxidation 

III. Chromium-saponite clay catalysts: Preparation, characterization and catalytic performance in propene oxidation

\section{Abstract}

Chromium-saponite catalysts have been prepared by two synthesis procedures. The first method consisted in the intercalation of the saponite with solutions containing aluminium and chromium oligomers with various molar ratios, while the second one consisted in the incipient wetness impregnation of alumina-pillared saponite with several chromium salts, ammonium chromate, $\operatorname{Cr}$ (II) acetate and $\mathrm{Cr}$ (III) nitrate. Several techniques, X-ray diffraction, nitrogen physisorption at $-196{ }^{\circ} \mathrm{C}$, thermogravimetric and differential thermal analysis, infrared and DR-UVVis spectroscopies, temperature-programmed reduction and electron paramagnetic resonance spectroscopy at $-196{ }^{\circ} \mathrm{C}$, have been used to characterize and to compare the properties of the materials synthesized. The results show that the solids obtained combine the layered structure of the clay and the thermal stability given by the alumina pillars. The catalysts have been tested in the oxidation of propene, showing a catalytic behaviour according to the Mars-van Krevelen mechanism, the performance not depending on the method in which chromium was incorporated to the clay.

Keywords: alumina-pillared clays, chromia-alumina-pillared clays, chromium catalysts, propene oxidation.

\section{I .1. I ntroduction}

Pillared interlayered clays (PILCs) are a very important family of porous solids that has been developed in the last years. The synthesis procedure includes three principal steps: i) the preparation of polyoxocations by hydrolysis of certain multivalent cations, which under appropriate conditions give rise to cationic polymeric species, ii) the formation of the intercalated clays by ion exchange of the original charge-compensating cations of swellable smectite clays by the polyoxocations synthesized in step (i), and iii) the formation of the pillared clays by calcination of the intercalated clays to high temperatures, at which the metastable polyoxocations are transformed into stable oxi-hydroxidic phases, which act as pillars that support the clay layers and maintain them separated. The clay layers prevent aggregation of the metallic oxide clusters, yielding to very dispersed particles. The porous structure of the pillared clays depends on the number and the size of the pillars in the interlayer region [1-7]. 
Intercalation of chromium is particularly interesting, because it can form welldefined polycations, such as a dimer $\left[\mathrm{Cr}_{2}(\mathrm{OH})_{2}\left(\mathrm{H}_{2} \mathrm{O}\right)_{8}\right]^{4+}$, a trimer $\left[\mathrm{Cr}_{3}(\mathrm{OH})_{4}\left(\mathrm{H}_{2} \mathrm{O}\right)_{9}\right]^{5+}$, two tetramers, an open one $\left[\mathrm{Cr}_{4}(\mathrm{OH})_{6}\left(\mathrm{H}_{2} \mathrm{O}\right)_{11}\right]^{6+}$ and a closed one $\left[\mathrm{Cr}_{4}(\mathrm{OH})_{5} \mathrm{O}\left(\mathrm{H}_{2} \mathrm{O}\right)_{10}\right]^{5+}$, or even a hexameric species [8-13]. These species coexist in $\mathrm{Cr}$-speciation diagram with the monomer $\left[\mathrm{Cr}\left(\mathrm{H}_{2} \mathrm{O}\right)_{6}\right]^{3+}$, and we may refer to all them with the general term "Cr-polycations", although the last one obviously is not a polymeric species. Aluminium can easily be polymerised to form the wellknown polycation $\left[\mathrm{Al}_{13} \mathrm{O}_{4}(\mathrm{OH})_{24}\left(\mathrm{H}_{2} \mathrm{O}\right)_{12}\right]^{7+}$, in short $\mathrm{Al}_{13}$, very efficient for the intercalation of clays [14-16]. Alumina-pillared clays are thus well-known, easy to prepare and very stable [1-7]. The joint hydrolysis of $\mathrm{Al}(\mathrm{III})$ and $\mathrm{Cr}$ (III) has as a result the incorporation of two elements to the structure of the clay [17-20]. Cointercalation can be carried out in a relatively easy way by the joint hydrolysis of the two elements, followed by the addition of the solution containing the polymerized cations to the clay. Although it has been claimed that $\mathrm{Cr}^{3+}$ cations may be incorporated in the hydrolysis to the structure of $\mathrm{Al}_{13}$ units $[20,21]$, no enough evidence of such mixed species has been found, and each cation may form its own polymeric species.

Chromium is an interesting metal for catalytic applications because of the variety of its oxidation states [22]. Chromium catalysts have been used in several reactions with environmental applications, such as the combustion of volatile organic compounds (VOCs) [23,24] and Cl-VOCs [25], and DeNOx reactions [26]. Only in a few cases these catalysts are based in clay supports [24,27]. Natural clays are not as common catalyst supports as alumina, silica, zeolites or carbon. The use of pillared clays, because of their relatively long preparation procedure, is justified if very specific catalysts are obtained $[28,29]$.

In the present paper, the preparation, characterization and catalytic behaviour of several chromia-saponite catalysts are reported. The samples have been prepared by co-pillaring of saponite with aluminium- and chromium-polycations, at various $\mathrm{Al} / \mathrm{Cr}$ ratios and by impregnation of alumina-pillared saponite with various chromium precursors. The pillared samples were evaluated as catalysts in the oxidation of propene, chosen as a common hydrocarbon test in exhaust treatments $[30,31]$. 


\section{I .2. Experimental}

III.2.1. Raw materials and preparation of the catalysts

The clay used in this work was a saponite from Yunclillos (Toledo, Spain), kindly supplied by TOLSA (Madrid, Spain). The raw material was purified by dispersion in water, careful decantation and extraction of the fraction with particle size smaller than $2 \mu \mathrm{m}$. The structural formula of purified saponite on the basis of an unit cell, 22 oxygen atoms, based on the chemical analysis given in Table 1, was found to be $\left[\begin{array}{llllll}\mathrm{Si}_{7.348} & \mathrm{Al}_{0.652}\end{array}\right]\left[\begin{array}{lllll}\mathrm{Al}_{0.178} & \mathrm{Mg}_{5.369} & \mathrm{Mn}_{0.004} & \mathrm{Fe}_{0.154} & \mathrm{Ti}_{0.022}\end{array} \mathrm{O}_{20}(\mathrm{OH})_{4}\left[\begin{array}{ll}\mathrm{Ca}_{0.096} & \mathrm{Na}_{0.066} \\ \mathrm{Mg}_{0.230}\end{array}\right.\right.$ $\left.\mathrm{K}_{0.081}\right]$. The cation exchange capacity (CEC) was 0.99 mequiv/g, the basal spacing $13.8 \AA$ and the BET specific surface area $169 \mathrm{~m}^{2} / \mathrm{g}$.

\begin{tabular}{ccccccccccc}
\hline Sample & $\mathrm{SiO}_{2}$ & $\mathrm{Al}_{2} \mathrm{O}_{3}$ & $\mathrm{Fe}_{2} \mathrm{O}_{3}$ & $\mathrm{MnO}$ & $\mathrm{MgO}$ & $\mathrm{CaO}$ & $\mathrm{Na}_{2} \mathrm{O}$ & $\mathrm{K}_{2} \mathrm{O}$ & $\mathrm{TiO}_{2}$ & $\mathrm{Cr}_{2} \mathrm{O}_{3}$ \\
\hline Saponite & 60.09 & 5.77 & 1.65 & 0.03 & 30.70 & 0.71 & 0.27 & 0.52 & 0.25 & 0.00 \\
$\mathrm{Al}_{1.0}$ & 56.46 & 14.56 & 1.51 & 0.03 & 26.64 & 0.11 & 0.04 & 0.44 & 0.22 & 0.00 \\
$\mathrm{Al}_{0.9} \mathrm{Cr}_{0.1}$ & 54.35 & 15.19 & 1.46 & 0.03 & 25.73 & 0.05 & 0.05 & 0.37 & 0.22 & 2.55 \\
$\mathrm{Al}_{0.5} \mathrm{Cr}_{0.5}$ & 52.40 & 10.66 & 1.41 & 0.03 & 24.20 & 0.04 & 0.04 & 0.44 & 0.22 & 10.55 \\
$\mathrm{Al}_{0.2} \mathrm{Cr}_{0.8}$ & 51.37 & 7.83 & 1.38 & 0.03 & 23.81 & 0.04 & 0.02 & 0.36 & 0.20 & 14.96 \\
$\mathrm{Cr}_{1.0}$ & 49.90 & 5.08 & 1.42 & 0.03 & 22.14 & 0.04 & 0.01 & 0.41 & 0.22 & 20.75 \\
$\mathrm{Cr}_{\text {ac }}$ & 51.67 & 13.58 & 1.45 & 0.03 & 24.19 & 0.10 & 0.02 & 0.33 & 0.22 & 8.39 \\
$\mathrm{Chromate}_{\text {nate }}$ & 51.99 & 13.36 & 1.40 & 0.03 & 24.34 & 0.09 & 0.01 & 0.35 & 0.21 & 8.22 \\
$\mathrm{Cr}_{\text {Nit }}$ & 51.90 & 13.57 & 1.41 & 0.03 & 24.49 & 0.10 & 0.04 & 0.42 & 0.22 & 7.82 \\
\hline
\end{tabular}

Table 1. Chemical analyses (wt. \%) of the natural, pillared and impregnated solids, referred to dry solids ( $0 \%$ water).

Saponite was intercalated with chromium, aluminium, and solutions containing both elements. Intercalation with chromium polycations was carried out following a procedure described previously [32,33]. According to this procedure, $30 \mathrm{mmol}$ of $\mathrm{CrCl}_{3} \cdot 6 \mathrm{H}_{2} \mathrm{O}$ (Aldrich, purissimum) were dissolved in water, and hydrolysed with 1 $\mathrm{mol} / \mathrm{dm}^{3} \mathrm{NaOH}$ (Panreac, purissimum), at a $\mathrm{OH}^{-} / \mathrm{Cr}^{3+}$ mole ratio equal to 2.0 . The solution thus obtained was aged for $72 \mathrm{~h}$ so that polymerisation took place and then added to a previously prepared suspension of saponite in water at a ratio of $5.0 \mathrm{mmol} \mathrm{Cr}^{3+} / \mathrm{g}_{\text {clay }}$. The resulting slurry was left under stirring for $72 \mathrm{~h}$ to favour cation exchange, and then washed by dialysis until absence of chloride. The intercalated solid was separated by centrifugation and dried at $70{ }^{\circ} \mathrm{C}$ for $16 \mathrm{~h}$. Finally, it was heated at $1^{\circ} \mathrm{C} / \mathrm{min}$ up to $500^{\circ} \mathrm{C}$ in an oven and calcined for $2 \mathrm{~h}$. 
Intercalation with aluminium polycations was carried out by a similar procedure $[15,16]$. First, $\mathrm{AlCl}_{3} \cdot 6 \mathrm{H}_{2} \mathrm{O}$ (Panreac, purissimum) was dissolved in water and hydrolysed with $1 \mathrm{~mol} / \mathrm{dm}^{3} \mathrm{NaOH}$ (Panreac, purissimum), at a $\mathrm{OH}^{-} / \mathrm{Al}^{3+}$ mole ratio equal to 2.2. The resulting solution was left under vigorous stirring for $24 \mathrm{~h}$ so that aluminium polycations $\left(\mathrm{Al}_{13}\right)$ were formed, and then added to a clay suspension, at a ratio of $5 \mathrm{mmol}_{\mathrm{Al}}{ }^{3+} / \mathrm{g}_{\text {clay }}$. The slurry was aged under stirring for $24 \mathrm{~h}$, and then washed by dialysis until absence of chloride. The solid was obtained by centrifugation, dried at $70{ }^{\circ} \mathrm{C}$ and calcined at $500{ }^{\circ} \mathrm{C}$ as the chromium intercalated clays.

For the preparation of the intercalating solutions containing the two elements, the required amounts of $\mathrm{AlCl}_{3} \cdot 6 \mathrm{H}_{2} \mathrm{O}$ and $\mathrm{CrCl}_{3} \cdot 6 \mathrm{H}_{2} \mathrm{O}$ were dissolved in water at three $\mathrm{Al} / \mathrm{Cr}$ mole ratios, specifically $0.9: 0.1 ; 0.5: 0.5$ and $0.2: 0.8$. The three solutions were hydrolysed with a $\mathrm{NaOH}$ solution having a concentration of $1 \mathrm{~mol} / \mathrm{dm}^{3}$ at $\left(\mathrm{OH}^{-} / \mathrm{Al}^{3+}+\mathrm{Cr}^{3+}\right)$ mole ratios of $2.2,2.1$ and 2.0, respectively. The rest of the preparation procedure was similar to the ones already described for the pillared clays containing only one metal [19].

The alumina-pillared solid, which may be considered as reference pillared clay, was used as the support for the preparation of the supported catalysts by incipient wetness impregnation. The salts used for the impregnation were ammonium chromate $\left(\mathrm{NH}_{4}\right)_{2} \mathrm{CrO}_{4}$ (Aldrich $98 \%$ ), chromium(II) acetate monohydrate dimer $\left[\mathrm{Cr}\left(\mathrm{CH}_{3} \mathrm{COO}\right)_{2} \cdot \mathrm{H}_{2} \mathrm{O}\right]_{2}$ (Aldrich) and chromium(III) nitrate nonahydrate $\mathrm{Cr}\left(\mathrm{NO}_{3}\right)_{3} \cdot 9 \mathrm{H}_{2} \mathrm{O}$, (Aldrich 99\%), which are precursors containing chromium in various oxidation states, that were dissolved in the minimum amount of water, in acetone in the case of the acetate, not soluble in water, and then added to the support. The resulting solids were dried and calcined in the same way as the pillared solids. In all cases, the final loading of the catalysts was $8 \mathrm{wt} . \%$ of $\mathrm{Cr}_{2} \mathrm{O}_{3}$.

The intercalated-pillared solids are hereafter referred as $\mathrm{Al}_{x} \mathrm{Cr}_{y}$, with $\mathrm{x}$ and $\mathrm{y}$ being the mole fraction of $\mathrm{Al}$ and $\mathrm{Cr}$, respectively. The clay-based solids are referred by the name of the precursor used for impregnation, namely $\mathrm{Cr}_{\mathrm{ac}}$, chromate and $\mathrm{Cr}_{\text {Nit }}$ stand for the catalysts prepared starting from $\mathrm{Cr}$ (II) acetate, ammonium chromate and $\mathrm{Cr}$ (III) nitrate, respectively. In all cases, these references correspond to the dried samples; the suffix "-500" is added to designate the calcined solids. 
III. Chromium-saponite clay catalysts: Preparation, characterization and catalytic performance in propene oxidation

\section{III.2.2. Characterization techniques}

X-ray diffraction (XRD) patterns of the solids were obtained over non-oriented powder samples, at a $2 \theta$ range from 2 to $65 \%$, scanned at $2 \% / \mathrm{min}$. The instrument used was a Siemens D-500 diffractometer with filtered $\mathrm{Cu} \mathrm{K \alpha}$ radiation operated at $40 \mathrm{kV}$ and $30 \mathrm{~mA}$.

Elemental analysis of the solids was carried out by inductively coupled plasma optical emission spectroscopy (ICP OES) at Activation Laboratories Ltd., Ancaster, Ontario, Canada.

Specific surface area and pore size distributions were obtained by static adsorption of nitrogen (Air Liquide, $99.9992 \%$ ) at $-196{ }^{\circ} \mathrm{C}$, using a static volumetric apparatus (Micromeritics ASAP 2010 adsorption analyser). About $0.1 \mathrm{~g}$ of sample was used for each experiment, previously outgassed at a pressure lower than $6.67 \mathrm{~Pa}$, first at room temperature for $6 \mathrm{~h}$ and then at $110{ }^{\circ} \mathrm{C}$ for $2 \mathrm{~h}$.

Thermal studies were performed on Perkin-Elmer analysers, TGA7 and DTA7 for thermogravimetric and differential thermal analysis, respectively. All measurements were carried out at a heating rate of $10{ }^{\circ} \mathrm{C} / \mathrm{min}$ under a flow of $20 \mathrm{~cm}^{3} / \mathrm{min}$ of air (Air Liquide, $99.999 \%$ ) or nitrogen (Air Liquide, $99.9992 \%$ ).

FT-IR spectra were recorded in the $350-4000 \mathrm{~cm}^{-1}$ region on a Perkin-Elmer 1730 Infrared Fourier Transform Spectrometer. About $1 \mathrm{mg}$ of sample and $300 \mathrm{mg}$ of $\mathrm{KBr}$ (Panreac, for IR spectroscopy) were used in the preparation of the pellets.

DR-UV-Vis spectra were recorded in reflectance mode on a Cary 5 spectrometer, with an optical length of $0.2 \mathrm{~cm}$.

Temperature-programmed reduction (TPR) analyses were carried out on a Micromeritics TPR/TPD 2900 instrument. About $40 \mathrm{mg}$ of sample were heated from room temperature to $900{ }^{\circ} \mathrm{C}$ at $10{ }^{\circ} \mathrm{C} / \mathrm{min}$, under a flow of $60 \mathrm{~cm}^{3} / \mathrm{min}$ of carrier gas ( $5 \% \mathrm{H}_{2}$ in $\mathrm{Ar}$, Air Liquide). Hydrogen consumption was measured by a thermal conductivity detector (TCD) and CuO (Merck, 99.99\%) was used as an external standard for area calibration. Water and other compounds that may be formed 
during the precursor decomposition and the metal reduction were retained in a cold trap to avoid any interference with the measured signal.

The electron paramagnetic resonance (EPR) spectra were recorded at $-196^{\circ} \mathrm{C}$ in a Bruker ER 200D spectrometer calibrated with DPPH (diphenyl picryl hydrazide). The samples were previously outgassed under vacuum conditions, ca. $26.610^{-3} \mathrm{~Pa}$, for $1.5 \mathrm{~h}$.

Catalytic tests were carried out in a fixed bed flow reactor. The catalysts were sieved and the fraction between 125 and $250 \mu \mathrm{m}$ was used in the catalytic experiments. The feed was adjusted in order to evaluate the oxidant capacity of several reaction mixtures, choosing respectively oxygen and nitrogen oxides as oxidant agents. The propene concentration was attuned to $0.1 \% \mathrm{C}_{3} \mathrm{H}_{6}(1.86 \%$ $\mathrm{C}_{3} \mathrm{H}_{6}$ in $\mathrm{Ar}$, Air Liquide) mixing with $0.45 \% \mathrm{O}_{2}\left(20 \% \mathrm{O}_{2}\right.$ in $\mathrm{N}_{2}$, Air Liquide) or $0.9 \%$ $\mathrm{NO}_{\times}\left(98 \% \mathrm{NO}+1 \% \mathrm{NO}_{2}+0.5 \mathrm{~N}_{2} \mathrm{O}\right.$, Carburos Metálicos) in argon (99.999\%, Air Liquide), establishing a total flow of $200 \mathrm{~cm}^{3} / \mathrm{min}$. The gas-hourly space velocity (GHSV) was fixed to $19000 \mathrm{~h}^{-1}$ after checking the optimum conditions to compare the catalysts. Propene oxidation was studied from 200 to $500{ }^{\circ} \mathrm{C}$, at intervals of $50{ }^{\circ} \mathrm{C}$. In all cases, the samples were raised to the measurement temperature with a temperature rate of $5 \mathrm{o} / \mathrm{min}$ and stabilized for $45 \mathrm{~min}$ prior to analysis, in order to ensure steady-state conditions. The reactants and products of the reaction were analysed by on-line infrared spectroscopy with a Perkin-Elmer 1725X FTIR spectrometer with a multiple reflection transmission cell (Infrared Analysis Inc.).

\section{III.3. Results and discussion}

\section{III.3.1. Characterization of the catalysts}

The solid intercalated with aluminium shows the X-ray diffractogram typical from alumina-pillared clays, see Fig. 1 , sample $\mathrm{Al}_{1.0}$. The basal spacing increases from $13.81 \AA$, in the natural clay (diffractogram not shown), to $18.33 \AA$ in the intercalated solid. Also intercalation with aluminium-chromium polycations produces a shift of the basal (001) reflection peak to lower angles, indicating an increase in the basal spacing, intercalation being effective in all the solids considered, see Fig. 1. More differences are observed for the solids calcined at $500{ }^{\circ} \mathrm{C}$, although all of them show a layered structure, solids where aluminium is the only or the 
majority element are well-ordered, while for solids where chromium is the only or the majority element, their ordering in the c-dimension is rather low. Literature data show that alumina-pillared clays are thermally stable, particularly aluminapillared saponites, for which maintenance of the layered structure has been reported under calcination up to $700^{\circ} \mathrm{C}$ [34]. However, chromia-pillared clays are thermally stable only up to $200^{\circ} \mathrm{C}$ [19]. Thus, for alumina-chromia-pillared clays, it is expected that alumina gives thermal stability to the solids, while increasing amounts of chromia will loss this stability. In XRD patterns, this effect may be observed as a total disappearance of $d_{001}$ reflection peak, or at least as a strong decrease in its intensity, showing relative ordering due to the regions where the layered structure may be maintained by alumina pillars. This is the trend actually found; the solid $\mathrm{Al}_{1.0}-500$ shows an intense $\mathrm{d}_{001}$ reflection at $18.48 \AA$, while the solid $\mathrm{Al}_{0.9} \mathrm{Cr}_{0.1}$ displays this reflection, also as an intense peak, at $17.21 \AA$. In opposite, the solid $\mathrm{Cr}_{1.0}-500$ shows a wide band between 10.05 and $12.92 \AA$. In the series where $\mathrm{Cr}$ is majority, $\mathrm{Cr}_{2} \mathrm{O}_{3}$-eskolaite peaks are detected in the solids calcined at $500{ }^{\circ} \mathrm{C}$.

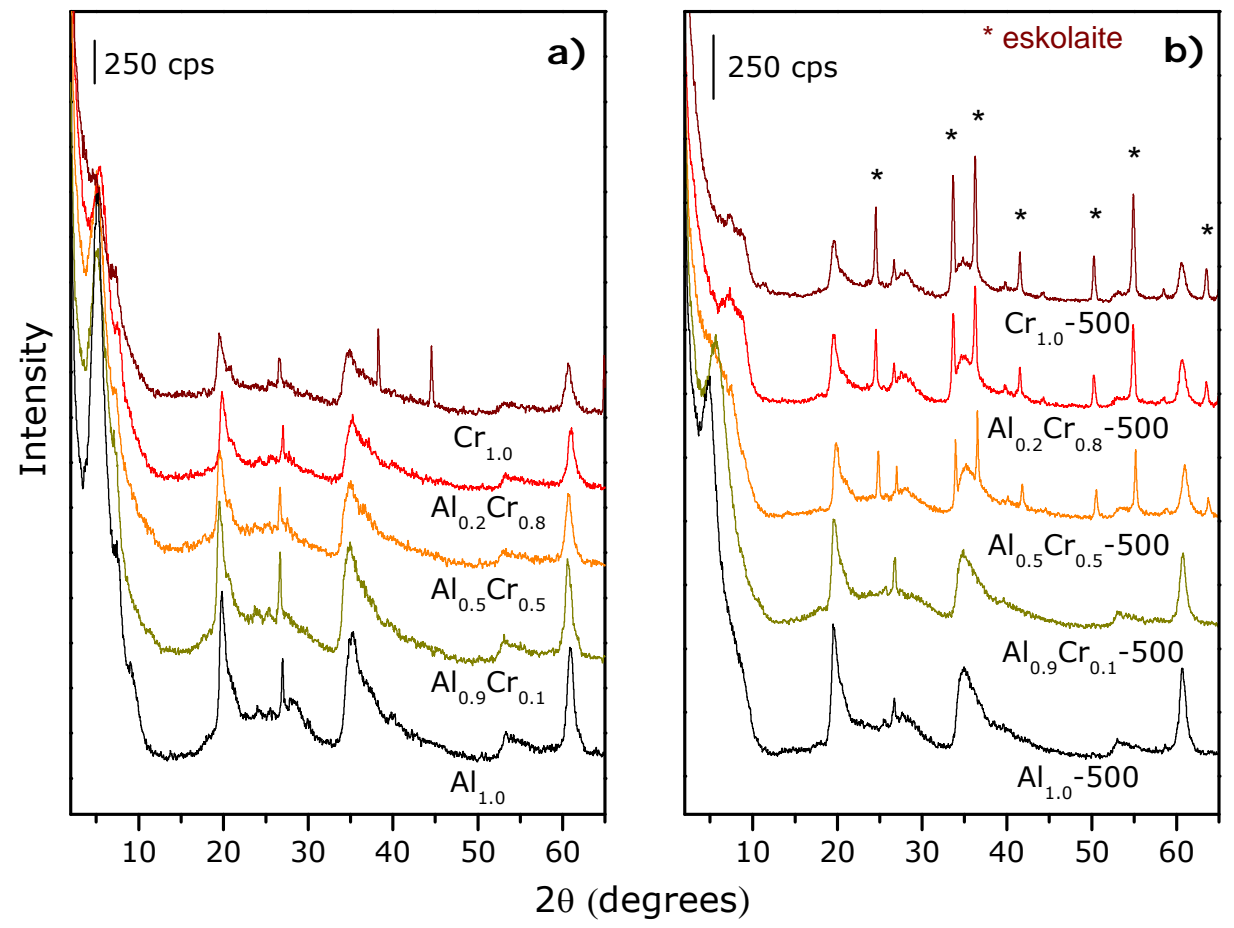

Fig. 1. XRD patterns of (a) intercalated and (b) pillared clays. 
As indicated before, the impregnated solids are prepared by using the saponite pillared with $\mathrm{Al}_{13}$ polycations, sample $\mathrm{Al}_{1.0}-500$, as support. The clay based solids maintain, in all cases, the layered structure of the support, see Fig. 2, although with a loss in the ordering in the c-axis. When considering the diffractograms of the solids after calcination, which is the final catalysts, c-axis ordering is low, solids being mainly delaminated. No peaks of chromium oxides neither oxihydroxides are detected, this suggesting a high dispersion of the chromium species on the surface of the support, forming amorphous species or particles with size below the detection limit of X-ray diffraction, which is particularly interesting considering the relatively high amount of chromium used for impregnation.

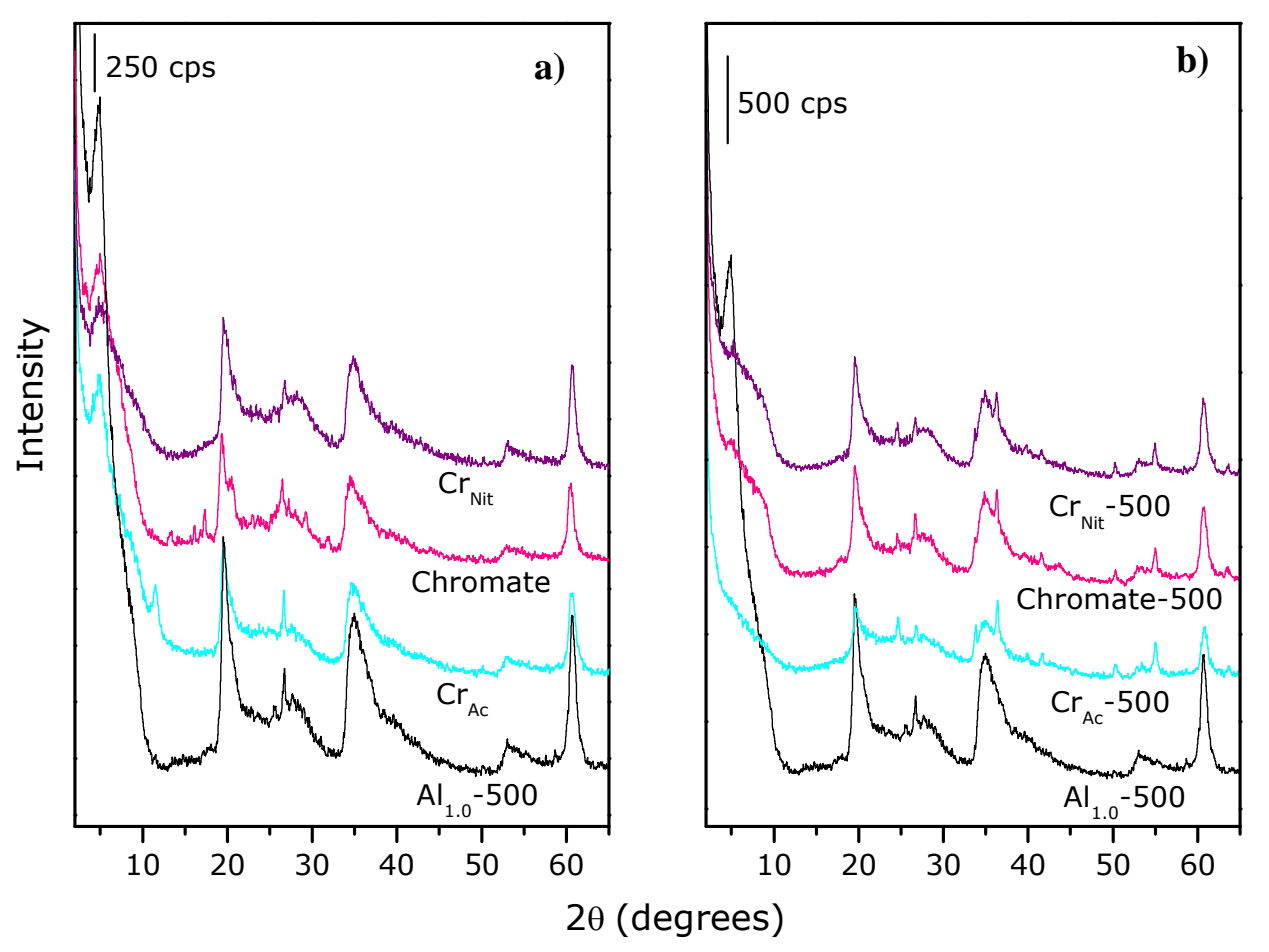

Fig. 2. XRD patterns of impregnated clays (a) before and (b) after calcination.

The chemical analysis of the pillared solids reflects the fixation of aluminium and chromium during the intercalation process, together with the evolution of other elements of the clay. The chemical composition of all the solids are given in Table 1, for better comparison expressed as water-free composition calculated considering the sum of the metallic oxides as $100 \%$. A strong decrease in the amount of $\mathrm{Ca}$ and $\mathrm{Na}$ in the natural clay may be noticed, confirming that the incorporation of aluminium and chromium is produced by a cation exchange 
reaction between the polymeric cationic species of these elements in solution and the exchangeable cations in the interlayer region of saponite. The amount of aluminium and chromium fixed varies almost linearly with the molar ratio of the two elements in the intercalating solutions, reaching high fixation amounts, mainly in the case of chromium. Variations are also observed in the amount of other elements, in some cases, these are only relative variations due to the high amounts of aluminium and chromium incorporated to the solids.

\begin{tabular}{ccccccccccc}
\hline Sample & $\mathrm{SiO}_{2}$ & $\mathrm{Al}_{2} \mathrm{O}_{3}$ & $\mathrm{Fe}_{2} \mathrm{O}_{3}$ & $\mathrm{MnO}$ & $\mathrm{MgO}$ & $\mathrm{CaO}$ & $\mathrm{Na}_{2} \mathrm{O}$ & $\mathrm{K}_{2} \mathrm{O}$ & $\mathrm{TiO}_{2}$ & $\mathrm{Cr}_{2} \mathrm{O}_{3}$ \\
\hline Saponite & 60.09 & 5.77 & 1.65 & 0.03 & 30.70 & 0.71 & 0.27 & 0.52 & 0.25 & 0.00 \\
$\mathrm{Al}_{1.0}$ & 60.09 & 15.50 & 1.61 & 0.03 & 28.35 & 0.12 & 0.04 & 0.47 & 0.24 & 0.00 \\
$\mathrm{Al}_{0.9} \mathrm{Cr}_{0.1}$ & 60.09 & 16.80 & 1.61 & 0.03 & 28.45 & 0.05 & 0.05 & 0.41 & 0.25 & 2.81 \\
$\mathrm{Al}_{0.5} \mathrm{Cr}_{0.5}$ & 60.09 & 12.23 & 1.62 & 0.03 & 27.76 & 0.04 & 0.04 & 0.51 & 0.25 & 12.10 \\
$\mathrm{Al}_{0.2} \mathrm{Cr}_{0.8}$ & 60.09 & 9.16 & 1.61 & 0.03 & 27.86 & 0.04 & 0.03 & 0.42 & 0.24 & 17.50 \\
$\mathrm{Cr}_{1.0}$ & 60.09 & 6.12 & 1.71 & 0.03 & 26.66 & 0.05 & 0.02 & 0.50 & 0.27 & 24.99 \\
\hline
\end{tabular}

Table 2. Effect of the chemical treatments on the composition of the pillared solids.

Composition of pillared solids is referred to the $\mathrm{SiO}_{2}$ content in the natural saponite. All compositions given in wt. \% and referred to dry solids ( $0 \%$ water).

In order to better compare these variations, all compositions are referred in Table 2 to a reference value, the amount of $\mathrm{SiO}_{2}$ in the natural saponite, $60.09 \%$, being selected, because silicium is not altered in the treatments here carried out. When compositions are compared in this basis, it can be observed that the amount of $\mathrm{Al}_{2} \mathrm{O}_{3}$ fixed varies between 3 and $11 \%$, while the amount of $\mathrm{Cr}_{2} \mathrm{O}_{3}$ fixed varies between 3 and $25 \%$. The amount of aluminium incorporated in the $\mathrm{Al}_{1.0}$ solid is in good accordance with the amount expected for compensating the CEC of the clay, assuming that this element is majority forming $\mathrm{Al}_{13}{ }^{7+}$ units $[35,36]$. However, in the case of chromium, the amount fixed by the intercalated solids is clearly higher than that necessary for compensating CEC considering the polycationic species that this element can form. It may thus be proposed that chromium forms other polymeric species, or that a certain amount of this element is fixed in the surface of the clay. It is clearly observed in the normalized composition that the content of $\mathrm{Mg}$ decreases in the intercalated solids. The high sensibility of saponite clay under acidic conditions is well known, being $\mathrm{Mg}(\mathrm{II})$ in its octahedral positions easily leached $[37,38]$. It may be taken into account that the $\mathrm{pH}$ of the intercalating solutions is close to 4 , and although their leaching effect on the clay may be attenuated because of the slightly alkaline character of the clay suspension, it 
provokes an important dissolution of octahedral $\mathrm{Mg}$, almost $25 \%$ of the total amount of this element in the most acidic sample $\left(\mathrm{Cr}_{1.0}\right)$, with the subsequent deleterious effect in the layered ordering of the clays.

The formation of one or other of the polycations of chromium cited above strongly depends on the $\mathrm{Al} / \mathrm{Cr}$ ratio. As it has been previously reported by our own group [19], the co-hydrolysis of both elements takes place with a competition for the hydroxyl groups added to the cation solutions. $\mathrm{Al}^{3+}$, because of its higher acidity, polymerises first, and thus $\mathrm{Cr}^{3+}$ polymerisation strongly depends on the limited amount of hydroxyl not employed for polymerisation of $\mathrm{Al}^{3+}$. This, together with the amount of hydroxyl added, between 2.0 and 2.2 of $\mathrm{OH} / \mathrm{metal}$ mole ratios, makes that higher is the ratio of $\mathrm{Al}^{3+}$ in the solution, lower is the amount of hydroxyl available for $\mathrm{Cr}^{3+}$ polymerisation and consequently, lower the degree of polymerisation of this cation. This degree of polymerisation can be easily followed by visible spectroscopy, by study of the position and relative intensity of the two bands appearing between 400 and $600 \mathrm{~nm}$ [8-13]. Under the conditions used in this study, the trimeric species predominates in the solutions with stoichiometry $\mathrm{Cr}_{1.0}$ and $\mathrm{Al}_{0.2} \mathrm{Cr}_{0.8}$, the dimer predominates in the solution with stoichiometry $\mathrm{Al}_{0.5} \mathrm{Cr}_{0.5}$, and the monomer in the solution $\mathrm{Al}_{0.9} \mathrm{Cr}_{0.1}$. The presence of these species in the intercalating solutions is compatible with the basal spacing observed by X-ray diffraction, when comparing the interlayer distance with the height of the polycations calculated by Volzone [33].

The chemical composition given in Table 1 shows that the impregnated solids have $\mathrm{Cr}_{2} \mathrm{O}_{3}$ contents close to $8 \mathrm{wt}$ \%, the content targeted during the synthesis. The differences with respect to this value are due to the degree of hydration of the solids in each step of the synthesis.

The nitrogen adsorption-desorption isotherms of the solids are shown in Fig. 3 and 4. As can be observed, a high amount of nitrogen is adsorbed at low relative pressure. All the isotherms are of type II from the IUPAC classification, with a hysteresis loop type H3, behaviour characteristic of layered materials with slit-like pores $[39,40]$. The structural parameters calculated from these isotherms are given in Table 3. Specific surface areas were obtained by the BET method, the external surface area by the t-method, the total pore volume was calculated from the 
III. Chromium-saponite clay catalysts: Preparation, characterization and catalytic performance in propene oxidation

amount of nitrogen adsorbed at a relative pressure of 0.99 , and the micropore size distributions by using the Horvath-Kawazoe method [41].

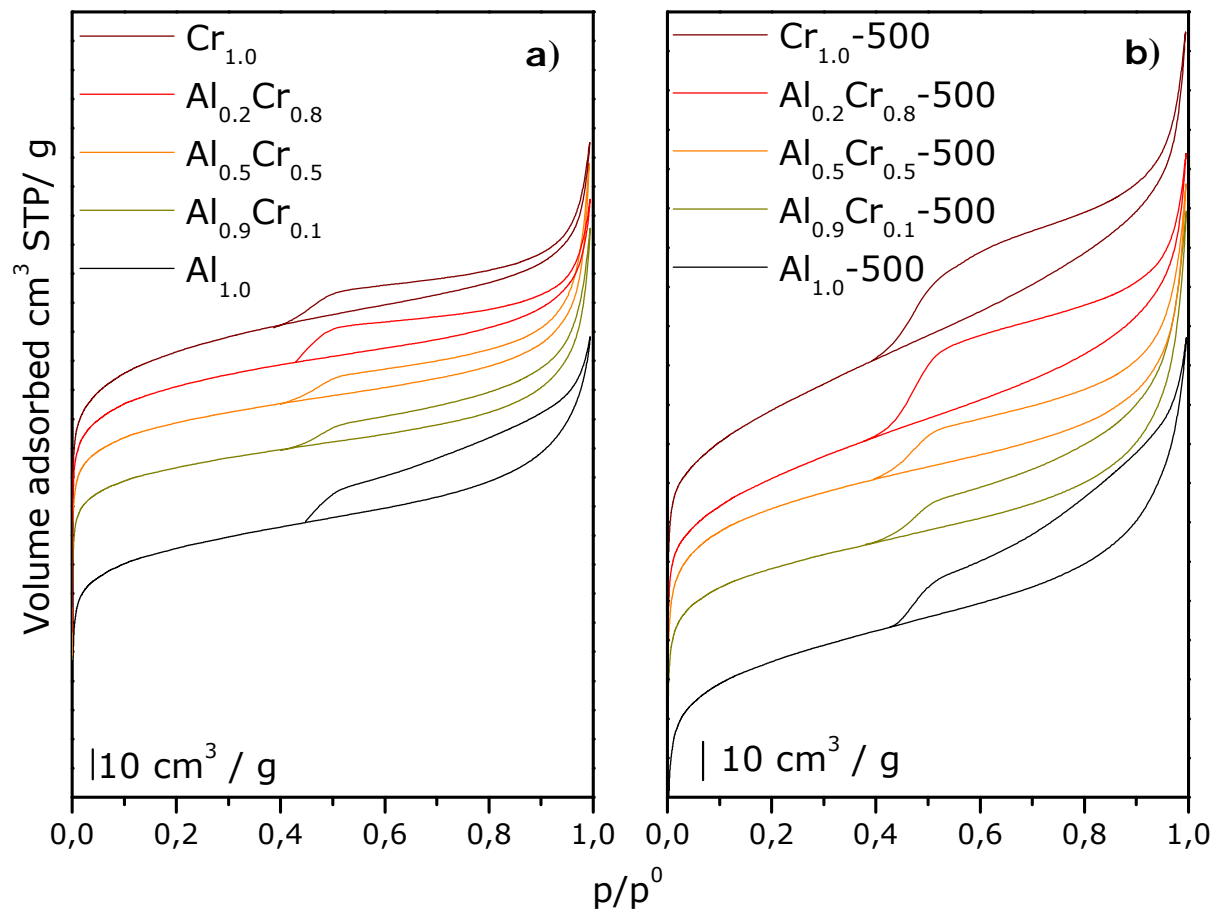

Fig. 3. Nitrogen adsorption-desorption isotherms at $-196{ }^{\circ} \mathrm{C}$ of (a) intercalated and (b) pillared clays.

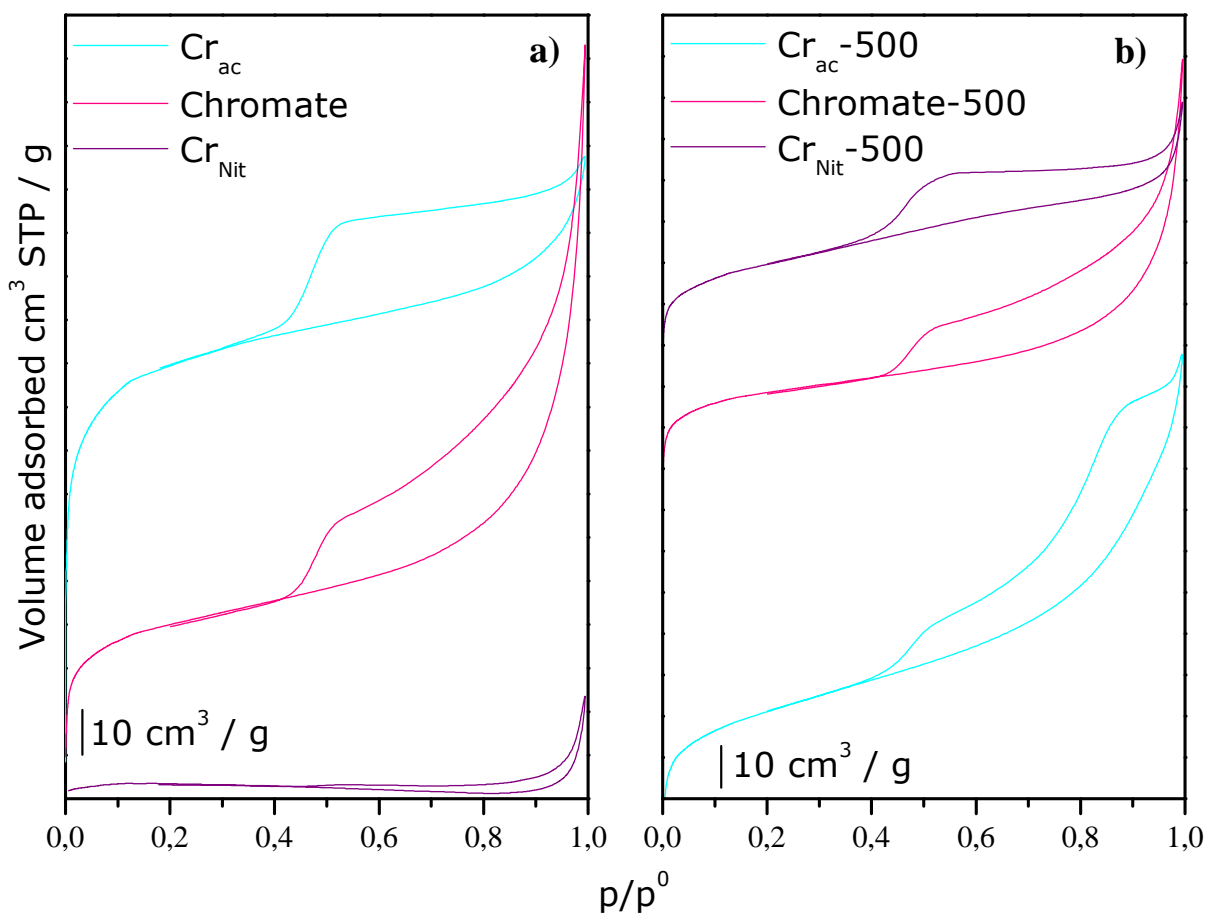

Fig. 4. Nitrogen adsorption-desorption isotherms at $-196^{\circ} \mathrm{C}$ of the impregnated clays, (a) before and (b) after calcination. 


\begin{tabular}{ccccccccc}
\hline Sample & \multicolumn{2}{c}{$\mathrm{S}_{\mathrm{BET}}\left(\mathrm{m}^{2} / \mathrm{g}\right)$} & \multicolumn{2}{c}{$\mathrm{S}_{\mu \mathrm{p}}\left(\mathrm{m}^{2} / \mathrm{g}\right)$} & \multicolumn{2}{c}{$\mathrm{Vp}\left(\mathrm{cm}^{3} / \mathrm{g}\right)$} & \multicolumn{3}{c}{$\begin{array}{c}\text { Micropore } \\
\text { diameter }(\AA)\end{array}$} \\
\cline { 2 - 9 } & $110^{\circ} \mathrm{C}$ & $500^{\circ} \mathrm{C}$ & $110^{\circ} \mathrm{C}$ & $500^{\circ} \mathrm{C}$ & $110^{\circ} \mathrm{C}$ & $500{ }^{\circ} \mathrm{C}$ & $110^{\circ} \mathrm{C}$ & $500{ }^{\circ} \mathrm{C}$ \\
\hline Saponite & 169 & & 111 & & 0.195 & & 5.5 \\
$\mathrm{Al}_{1.0}$ & 334 & 274 & 283 & 218 & 0.244 & 0.230 & 6.0 & 5.5 \\
$\mathrm{Al}_{0.9} \mathrm{Cr}_{0.1}$ & 258 & 240 & 217 & 191 & 0.220 & 0.231 & 5.3 & 5.4 \\
$\mathrm{Al}_{0.5} \mathrm{Cr}_{0.5}$ & 311 & 252 & 270 & 207 & 0.260 & 0.224 & 5.4 & 5.4 \\
$\mathrm{Al}_{0.2} \mathrm{Cr}_{0.8}$ & 327 & 191 & 285 & 120 & 0.226 & 0.202 & 5.4 & $5.9-8.8$ \\
$\mathrm{Cr}_{1.0}$ & 328 & 209 & 280 & 117 & 0.243 & 0.231 & 5.4 & $5.9-8.7$ \\
$\mathrm{Cr}_{\text {ac }}$ & 187 & 177 & 168 & 105 & 0.112 & 0.157 & 5.5 & 5.8 \\
$\mathrm{Chromate}$ & 72 & 97 & 44 & 61 & 0.015 & 0.257 & 8.0 & $7.0-8.9$ \\
$\mathrm{Cr}_{\text {Nit }}$ & 6 & 107 & n.a. ${ }^{a}$ & 73 & 0.094 & 0.251 & n.a. & $6.5-9.4$ \\
\hline a not applicable. & & & & & & &
\end{tabular}

Table 3. BET surface area $\left(\mathrm{S}_{\mathrm{BET}}\right)$, micropore surface area $\left(\mathrm{S}_{\mu \mathrm{p}}\right)$, total pore volume $(\mathrm{Vp})$, and micropore diameter of the solids.

First of all, it may be noticed that the specific surface area of the natural saponite is rather high, clearly higher than the values usually reported for natural smectites in general, and for saponites in particular $\left(30-50 \mathrm{~m}^{2} / \mathrm{g}\right)$. However, high BET surface area values have been reported by several authors for saponites in Madrid Basin, which has been attributed to the very small particle size of these clays due to their sedimentary origin $[37,38,42]$. Surface area shows the higher values for the solids intercalated with solutions containing only one cation, $\mathrm{Al}^{3+}$ or $\mathrm{Cr}^{3+}$, while solids derived from mixed solutions show lower values, although the decrease is only remarkable for $\mathrm{Al}_{0.9} \mathrm{Cr}_{0.1}$ solid.

Thermal treatment at $500{ }^{\circ} \mathrm{C}$ affects the solids depending on their composition. Thus, solids $\mathrm{Al}_{1.0}-500, \mathrm{Al}_{0.9} \mathrm{Cr}_{0.1}-500$ and even $\mathrm{Al}_{0.5} \mathrm{Cr}_{0.5}-500$ maintain a high percentage of the surface area of the corresponding counterparts before calcination, between 81 and $93 \%$. A loss of 42 and $37 \%$ of the surface area of the intercalated solids is observed in the samples $\mathrm{Al}_{0.2} \mathrm{Cr}_{0.8}$ and $\mathrm{Cr}_{1.0}$, respectively. This is related, to a large extent, to the thermal stability of the solids under calcination, as previously discussed when reporting their XRD data, the high collapse of chromium-rich solids under calcination at $500{ }^{\circ} \mathrm{C}$ entails a strong loss in surface area, while the maintenance of an ordered layered structure in the aluminium-rich solids causes the maintenance of most of surface area. Surface area always losses 
because calcination at $500{ }^{\circ} \mathrm{C}$ entails the removal of water from the polycationic species, thus provoking a decrease in the length of pillaring unities, or even the collapse of the layered structure observed by X-ray diffraction. The microporous surface contributes in a very significant way to the total surface area of the solids, reaching values between 84 and $87 \%$ in the intercalated solids and between 56 and $82 \%$ in the pillared counterparts.

The specific surface area of the materials leads very different results for the dried and the calcined impregnated solids. The solids dried at $70{ }^{\circ} \mathrm{C}$ show strong decreases of surface area with respect to the support, showing values as low as $6 \mathrm{~m}^{2} / \mathrm{g}$, even lower than the external surface area of the clay particles, meaning that the chromium salt has not only occluded the intraparticular porosity, but is acting as a kind of glue sticking the particles together. When the solids are calcined at $500{ }^{\circ} \mathrm{C}$ the surface area increases. The incorporation of the precursors, in some cases containing big organic moieties, strongly blocks the access to the interlayer microporous region of the support, causing the commented decrease in the surface area. The calcination of the solids to form the final catalysts causes the removal of the precursor components, and consequently opening the access to the previously blocked internal porosity. At this respect, the thermal curves show the decomposition of the organic moieties of the precursors as very exothermal processes centred at about $380{ }^{\circ} \mathrm{C}$, and the FT-IR spectra of the final catalysts, calcined at $500{ }^{\circ} \mathrm{C}$, confirms the absence of organic matter in them. For the nitrate precursor, no evidence of bands related to nitrate species appears in the corresponding FT-IR spectra of the $\mathrm{Cr}_{\mathrm{Nit}}-500$ sample indicating the removal of the nitrate precursor component. In any case, because of the incorporation of chromium and the commented partial collapse of the layered support structure during the impregnation-drying-calcination processes, the surface area of the supported catalysts is always lower than that of the support.

The total pore volume, determined from the amount of nitrogen adsorbed at a relative pressure of 0.99 is rather high, reaching for some solids values higher than $0.20 \mathrm{~cm}^{3} / \mathrm{g}$, probably related to interparticular porosity. The micropore size distribution of the solids, Fig. 5, determined by the Horvath-Kawazoe method, show that most of the solids have a monomodal pore distribution, with a maximum centred at 5.3-6.0 $\AA$. Some of the solids calcined at $500{ }^{\circ} \mathrm{C}$ present a bimodal distribution, with maxima close to 5.9 and $9.4 \AA$. This bimodal distribution has been 
previously reported for pillared clays [43], and as observed is maintained by some of the impregnated solids now prepared.

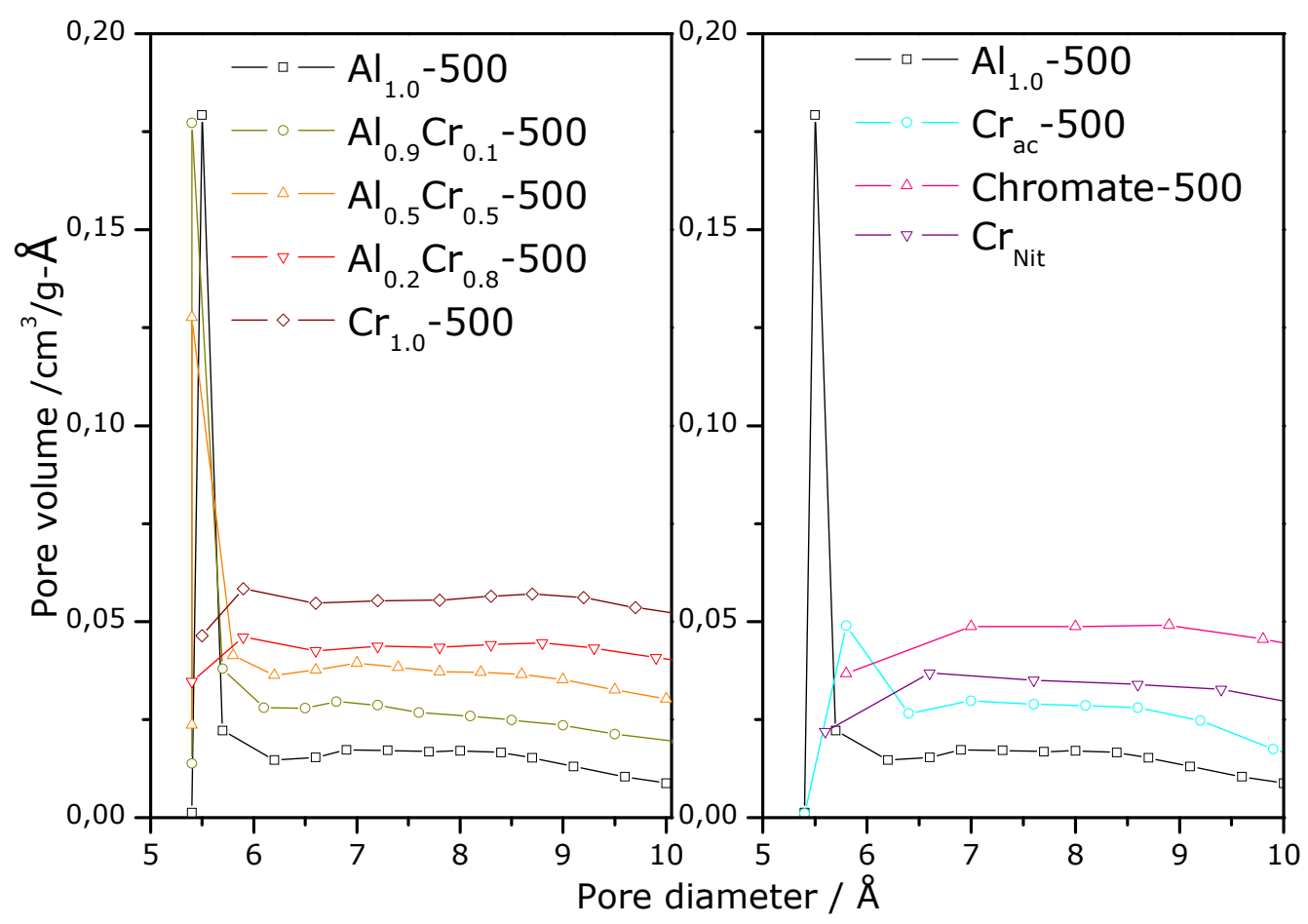

Fig. 5. Micropore size distribution for (a) pillared and (b) impregnated clays.

The DR-UV-Vis spectra of the solids show the characteristic bands of $\operatorname{Cr}$ (III) in octahedral environment, see Fig. 6. This cation shows three spin-allowed bands, usually two of them appearing in the Vis region ( $v_{1}$ and $v_{2}$ ) and the other appearing overlapped by the strong charge transference in the UV region $\left(v_{3}\right)$, and also a spinforbidden transition called the "ruby line" $\left(v_{4}\right)$. The spectra of the intercalated and pillared solids are very similar, with the visible bands centred at about 430 and $600 \mathrm{~nm}$ in the intercalated solids and at 460 and $600 \mathrm{~nm}$ for the pillared ones. The small shift observed in $v_{2}$ to high wavelength, that is, to low energy, for the pillared solids, and the subsequent decrease in the Racah parameters (not given) indicates that the pillared solids are more covalent that the intercalated ones, which accords with the dehydration and dehydroxilation that encompasses the calcination step carried out to transform the intercalated solids into the pillared ones, in other words, to transform the polycations into pillars [44]. As indicated, the impregnated solids show spectra rather different from the dried to the calcined solids, being remarkable that the spectra of the calcined solids are similar to those of the pillared 92 
solids, both series calcined at $500^{\circ} \mathrm{C}$. No bands that may be due to oxidation states different from $\mathrm{Cr}$ (III) were observed, although it may be noticed that $\mathrm{Cr}$ (VI) species do not present $d-d$ transitions. Thus, having in mind the limitation commented about the fact that Visible spectroscopy is blind to $\mathrm{Cr}(\mathrm{VI})$ species, it may be said that, independently of the precursor used in the impregnation the further treatments, particularly the calcination at $500{ }^{\circ} \mathrm{C}$, make all the final catalysts to contain similar chromium species.

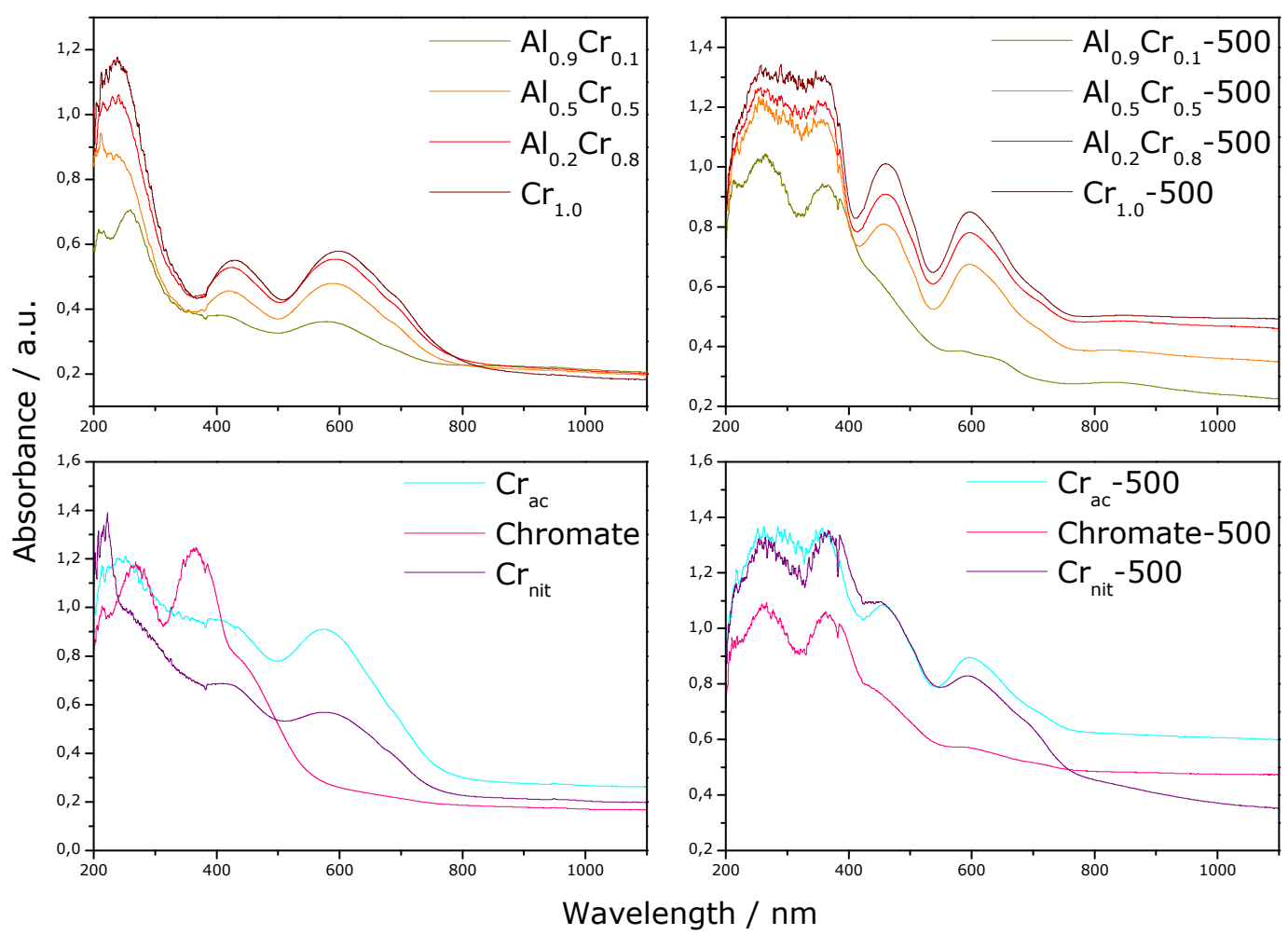

Fig. 6. DR-UV-Vis spectra of intercalated-pillared and of impregnated clays.

Temperature programmed reduction of the final catalysts shows that most of chromium in the solids is in the trivalent state. The reduction profiles, see Fig. 7, of natural saponite and of the $\mathrm{Al}_{1.0}-500$ solid present a small reduction effect at high temperature due to the small amount of $\mathrm{Fe}(\mathrm{III})$ in the octahedral positions of the saponite. Besides of this effect, the solids containing chromium present two reduction effects, the first one centred at 390-400 ${ }^{\circ} \mathrm{C}$ in the pillared solids and at 375-390 ${ }^{\circ} \mathrm{C}$ in the impregnated solids, and the second one appearing as a shoulder of the former, at $475{ }^{\circ} \mathrm{C}$. It has been reported that the calcination of solids containing chromium under oxidant atmosphere produces $\operatorname{Cr}(\mathrm{VI})$ species at moderate temperature, which decompose at higher temperature giving $\mathrm{Cr}$ (III) 
species. The amount of $\mathrm{Cr}(\mathrm{VI})$ has been reported to be close to $100 \%$ in some solids after calcination at $400{ }^{\circ} \mathrm{C}$, but strongly decreasing when the calcination temperature increases to $500{ }^{\circ} \mathrm{C}$ [45]. Taking in consideration that our solids have been calcined at $500^{\circ} \mathrm{C}$ in air, it is not reasonable to expect that chromium may be in the solids as $\mathrm{Cr}$ (II), and if it is as $\mathrm{Cr}$ (VI) it may be easily reduced up to $\mathrm{Cr}$ (III) by hydrogen, this reduction not continuing to $\mathrm{Cr}$ (II).

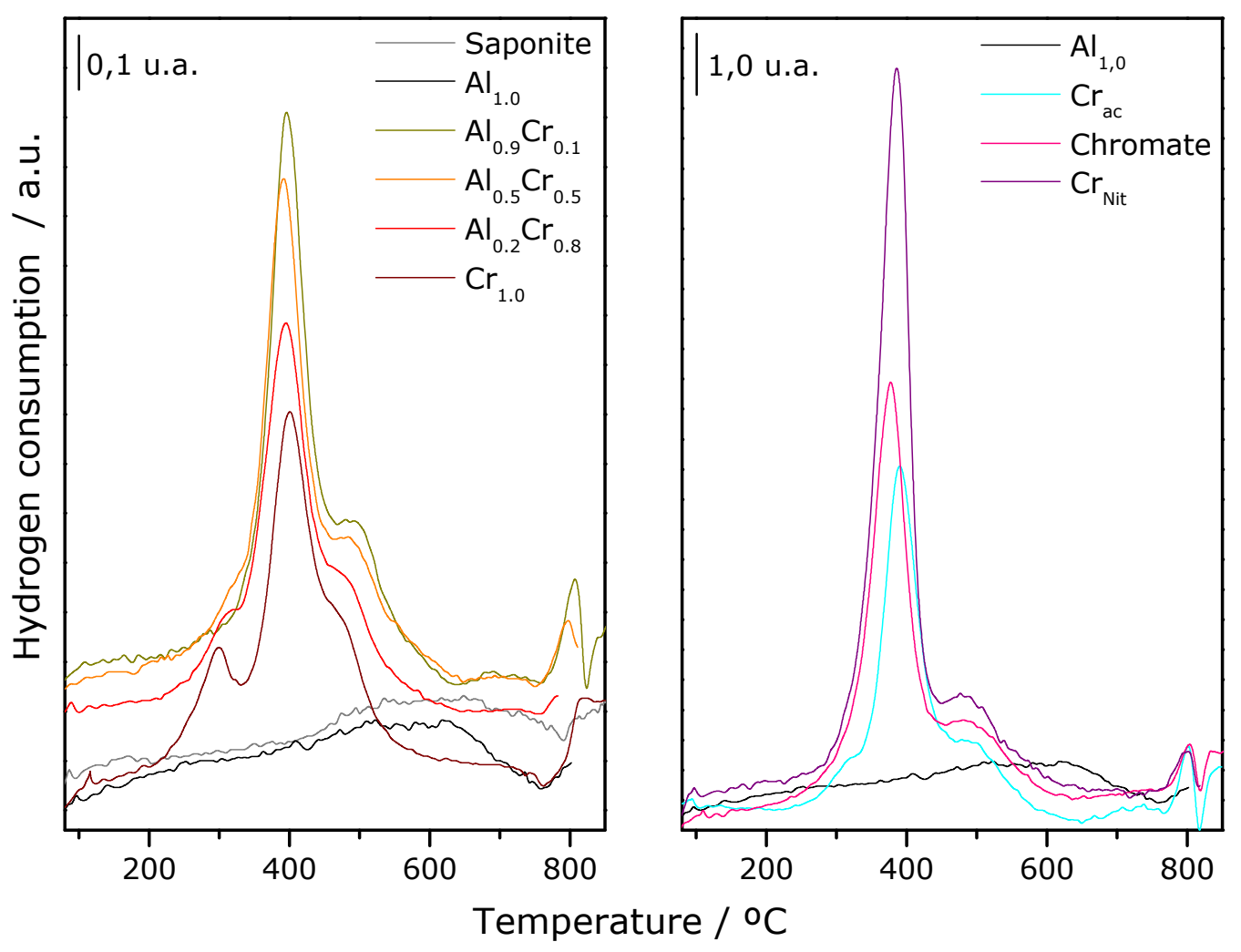

Fig. 7. TPR curves of (left) pillared and (right) impregnated clays.

The TPR curves of the impregnated catalysts are similar to that of $\mathrm{CrO}_{3}$. This suggests that the $\mathrm{Cr}(\mathrm{VI})$ species present in the solids are close to $\mathrm{Cr}(\mathrm{VI})$ oxide, even when ammonium chromate was used as precursor. Assuming that the reduction effects are due to the process $\mathrm{Cr}(\mathrm{VI}) \rightarrow \mathrm{Cr}$ (III) and the absence of other reduction processes, they allow to calculate the amount of chromium present in the solids calcined at $500{ }^{\circ} \mathrm{C}$ as $\mathrm{Cr}(\mathrm{VI})$ species, data given in Table 4, or alternatively to calculate an average oxidation state for chromium $[45,46]$. It was found that between 5.4 and $58.2 \%$ of total chromium is under the form of $\mathrm{Cr}(\mathrm{VI})$ species. However, it may be considered that the value of $58.2 \%$ of $\mathrm{Cr}(\mathrm{VI})$ is found for solid $\mathrm{Al}_{0.9} \mathrm{Cr}_{0.1}-500$, and because of the low amount of $\mathrm{Cr}$ fixed by this solid (see Table 1 ), 
the determination of the amount of $\mathrm{Cr}(\mathrm{VI})$ in this solid from hydrogen consumption has a high relative error. In the pillared solids, when higher is the amount of chromium fixed during the intercalation, lower is the percentage of $\mathrm{Cr}(\mathrm{VI})$ after calcination. In the impregnated solids, although the amount of chromium is very similar in all of them, the percentage of $\mathrm{Cr}$ in the $\mathrm{Cr}(\mathrm{VI})$ form varies between 16.6 and $34.4 \%$, suggesting that the amount of $\mathrm{Cr}(\mathrm{VI})$ after calcination at $500{ }^{\circ} \mathrm{C}$ depends on the nature of the precursor used. The amount of chromium that remains in the $\mathrm{Cr}(\mathrm{VI})$ form after calcination at $500{ }^{\circ} \mathrm{C}$ is clearly higher than that reported for other solids, in which even all chromium is in the trivalent form after calcination at this temperature [45].

\begin{tabular}{cc}
\hline Sample & $\begin{array}{c}\mathrm{Cr}(\mathrm{VI}) \text { content } \\
(\%)\end{array}$ \\
\hline $\mathrm{Al}_{0.9} \mathrm{Cr}_{0.1}$ & 58.2 \\
$\mathrm{Al}_{0.5} \mathrm{Cr}_{0.5}$ & 14.3 \\
$\mathrm{Al}_{0.2} \mathrm{Cr}_{0.8}$ & 7.3 \\
$\mathrm{Cr}_{1.0}$ & 5.4 \\
$\mathrm{Cr}_{\mathrm{ac}}$ & 16.6 \\
$\mathrm{Chromate}$ & 21.4 \\
$\mathrm{Cr}_{\text {Nit }}$ & 34.4 \\
\hline
\end{tabular}

Table 4. Percentage of $\mathrm{Cr}(\mathrm{VI})$ related to the total content of $\mathrm{Cr}$ in the solids calcined at $500^{\circ} \mathrm{C}$.

The characterization of chromium in the catalysts studied was completed by EPR measurements, which spectra are shown in Fig. 8. There are some reports describing the EPR investigations of chromium species on several oxide surfaces $[47,48]$. Mainly, three signals have been found in the EPR spectra of Cr-catalysts denoted as $Y, \beta$ and $\delta$. The axial $\gamma$-signal is centred at a $g$-factor value of 1.9 and it has been assigned to isolated $\operatorname{Cr}(V)$ species $[49,50]$. Whereas, the $\beta$-signal is attributed to $\mathrm{Cr}_{2} \mathrm{O}_{3}$-like clusters and the $\delta$-signal is associated to isolated or dispersed $\mathrm{Cr}$ (III) species [51]. Although again EPR can not detect $\mathrm{Cr}$ (VI) species and thus we can not infer results about species with this oxidation state from this technique, the presence and nature of these signals are dependent to the synthesis procedure, the support and the loading. 


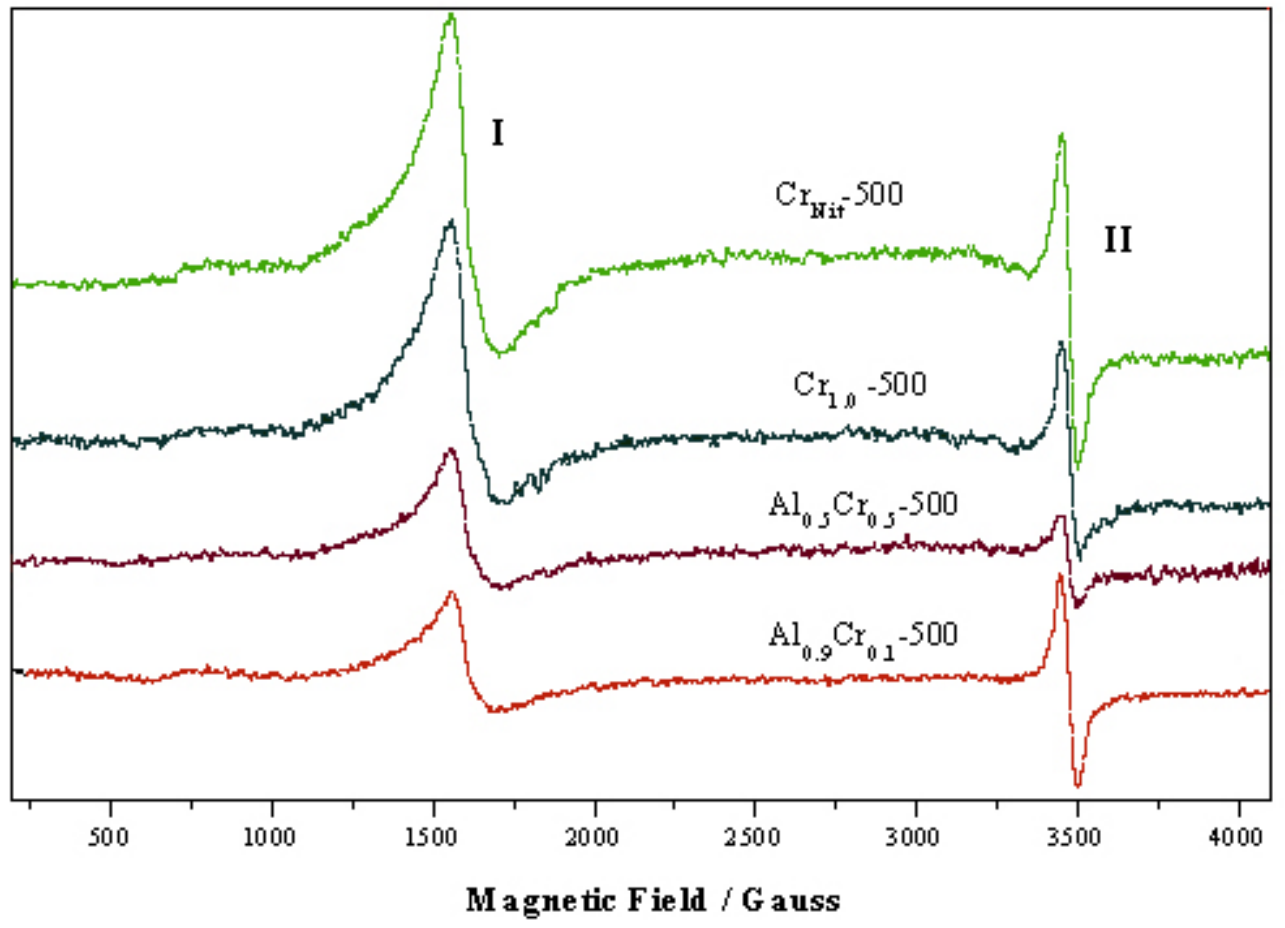

Fig. 8. EPR spectra of the samples indicated recorded at $-196{ }^{\circ} \mathrm{C}$ after outgassing.

The EPR spectra of all catalysts studied present the same two signals, denoted as $\mathbf{I}$ and $\mathbf{I} \mathbf{I}$. The signal $\mathbf{I}$ is centred at a $\mathrm{g}$ of 4.2 with a shoulder located at a $\mathrm{g}$ of 9 . It has an axial shape and it can be attributed to the presence of isolated octahedral $\mathrm{Fe}^{3+}$ species subjected to a rhombic distortion [52]. These iron species are located in the octahedral layer of the original clay, whose presence has been indicated previously in Table 1 . Signal $\mathbf{I I}$ is a symmetrical line centred at a $\mathrm{g}$ of 1.97 whose assignation offers some controversy. Depending on the support, at this position could appear the line assigned to $\operatorname{Cr}(\mathrm{V})$, denoted $\gamma$-signal, but also could be assigned to the presence of $\mathrm{Cr}$ (III) as $\mathrm{a}-\mathrm{Cr}_{2} \mathrm{O}_{3}$, signal $\beta[47,51]$. It is also important to remark that the literature also proposed the hypothesis of a trimer of mixed valence $\left(\mathrm{Cr}^{6+}-\mathrm{Cr}^{3+}-\mathrm{Cr}^{6+}\right)$ with average oxidation state of 5 as $\mathrm{Y}$-signal assignation [47]. In order to complete the EPR characterization, the same spectra were recorded at room temperature (not shown). The intensity of signal II decreased when the annealing temperature increased. This change is characteristic of the transformation of the paramagnetic species into non-paramagnetic species. This reduction in the intensity has been related to $\mathrm{Cr}_{2} \mathrm{O}_{3}$, which is weakly paramagnetic, being the dipolar interaction more notable than the interchange interaction at high temperatures [53]. In this sense, the characterization of chromium-saponite 96 
III. Chromium-saponite clay catalysts: Preparation, characterization and catalytic performance in propene oxidation

performed by various techniques confirms the presence of $\operatorname{Cr}$ (III) and $\operatorname{Cr}(\mathrm{VI})$ species in all the catalysts studied.

\section{III.3.2. Catalytic performance}

Selected solids were first tested for the catalytic reduction of NOx with propene, showing low activity for the nitrogen oxides reduction although high activity for the hydrocarbon oxidation, thus, we focused our attention on their catalytic behaviour for oxidation of propene. However, some results obtained in the NOx reaction are useful for explaining the catalytic performance of the solids.

The results obtained for the oxidation of propene, in presence of oxygen, are shown in Fig. 9. The conversion of propene reaches values of $100 \%$ for all the catalysts studied at $400{ }^{\circ} \mathrm{C}$, independently of the amount of chromium incorporated to the clay in the pillaring or impregnation processes. The effect of chromium amount only is apparent at lower temperatures. At $300{ }^{\circ} \mathrm{C}$ the increase of chromium percent slightly improves the propene conversion. This behaviour suggests that low amounts of chromium are effective for the propene oxidation reaction. As reference, the support used for the preparation of the impregnated solids, aluminium-pillared sample $\left(\mathrm{Al}_{1.0}-500\right)$, was included in Fig. 9 in order to evaluate its influence in the catalytic behaviour. The catalytic performance of this sample is very low, even at high temperatures, indicating that the behaviour of the catalysts evaluated is mainly due to the chromium species incorporated to the pillared clay.

The pillared and the impregnated solids have the same behaviour, as shown in Fig. 9, indicating that the catalytic performance does not depend on the method in which chromium was incorporated. This element acts as the active phase in oxidation reactions both if it is mainly located in the interlayer region or on the external surface of the clay. The propene combustion is not complete, as it has been described in literature for other chromium combustion catalysts [23]. At $350{ }^{\circ} \mathrm{C}, \mathrm{CO}$ is detected together with $\mathrm{CO}_{2}$ as reaction products. The $\mathrm{CO}$ selectivity reached at $500{ }^{\circ} \mathrm{C}$ is $40 \%$ for all the samples studied, except for the $\mathrm{Cr}_{1.0}-500$ sample, which reached $60 \%$ CO selectivity, see Fig. 9 . 

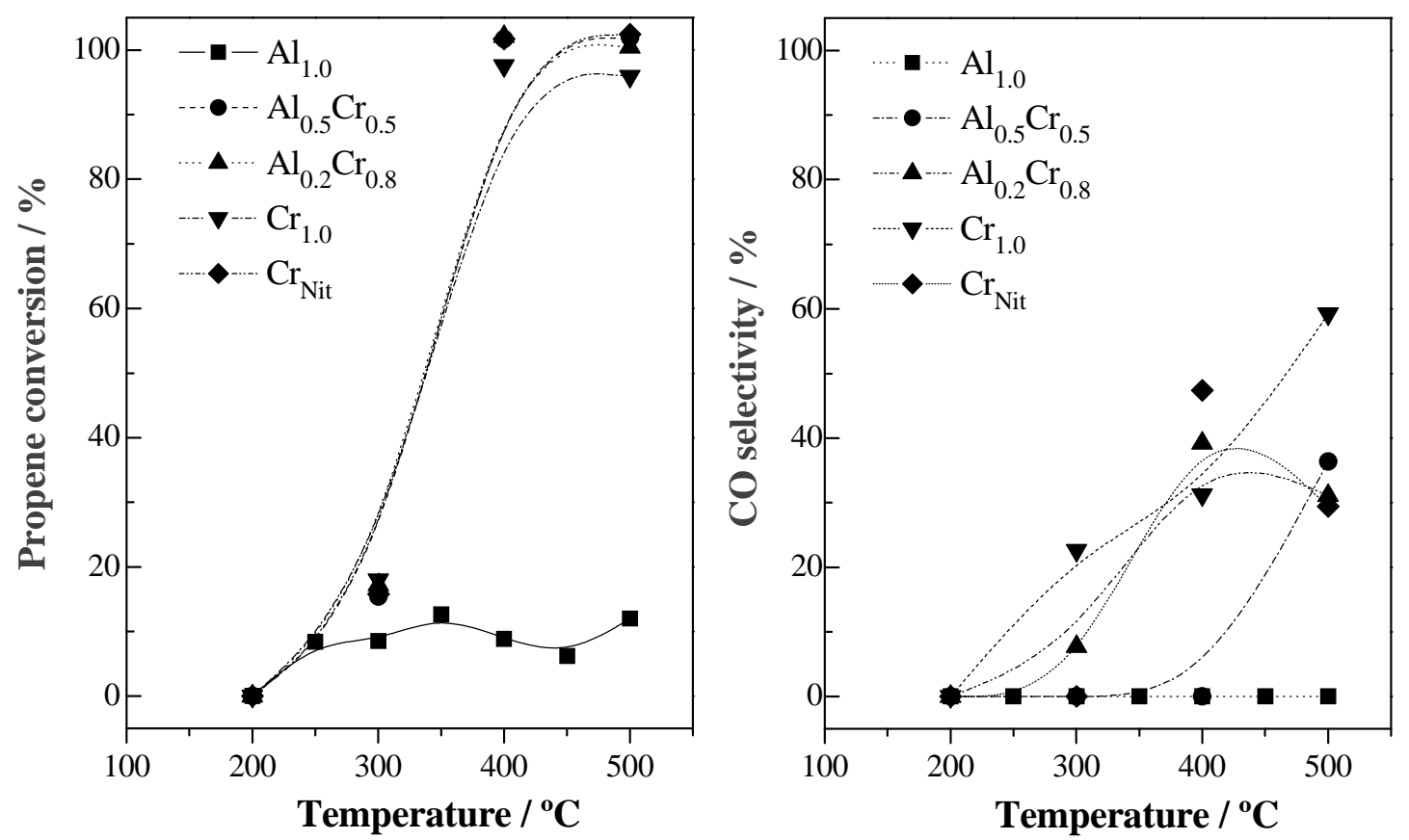

Fig. 9. Conversion of propene and selectivity to $\mathrm{CO}$ for propene oxidation with $\mathrm{O}_{2}$ oxidant.

The hydrocarbon oxidation begins at low temperatures, $250^{\circ} \mathrm{C}$, in all the solids studied. The reaction is activated from 300 to $400{ }^{\circ} \mathrm{C}$, reaching the maximum at this last temperature. This temperature should be related with the reduction peak observed in the $\mathrm{H}_{2}$-TPR measurements described before, attributed to the $\mathrm{Cr}(\mathrm{VI}) \rightarrow$ $\mathrm{Cr}$ (III) reduction. Thus, the activity of these materials could be attributed to the easy oxidation of $\mathrm{Cr}^{3+}$ ions to $\mathrm{Cr}^{6+}$ on the surface of chromium oxides. Similar relation between the activity and the TPR results has been described in the literature based on metal oxide catalysts for hydrocarbon combustion [54]. The two oxidation states are present in the chromium catalysts studied, as it has been described before by TPR, EPR and UV-Vis measurements. Chromium could present multiple oxidation states by an attachment to extra-lattice oxygen atoms. Due to its large atomic radius, the electron in its outermost shell (3d) is relatively easy to give high oxidation states and, thus, produce high redox potential. It is expected that the increase in oxidation state cause the increase of the electron accepting ability of chromium $[23,55]$. The high oxidation state is suitable to be reduced by organic compounds, giving low oxidation degrees capable to be oxidized by the oxygen, thus, closing the redox cycle. 
When using NO as oxidant agent, the catalysts behaviour was considerably different to that observed when using oxygen as oxidant, shown in Figure 10A. The oxidation of propene reached conversions close to $80 \%$ at high temperature, when pillared and impregnated catalysts are used. However, the conversion of NO reached values close to $30 \%$, that is, much lower than those reached for propene conversions. The yield to diatomic nitrogen under these conditions results analogous to $\mathrm{NO}_{\mathrm{x}}$ conversions for all catalysts studied. As there are no other oxidant agents in the feed, these results suggest that the catalysts contribute to the oxidation of the hydrocarbon by means of a Mars-van Krevelen mechanism. Assuming the Mars-van Krevelen when using NO as oxidant, the high oxidation activity of the chromium-catalysts for propene oxidation can also be explained by the Mars-van Krevelen model $[56,57]$, described in literature as the most suitable to represent the VOC's combustion reaction $[54,58]$.

Considering the behaviour observed when using oxygen or nitrogen oxide as oxidants, the reaction was carried out in the joint presence of both oxidants. The stoichiometric ratio propene-oxidants were maintained, making that the two oxidants give the same amount of oxygen atoms $\left(\mathrm{C}_{3} \mathrm{H}_{6}+4.5 \mathrm{NO}+2.25 \mathrm{O}_{2}\right)$. The conversion of propene found under these conditions reached higher values than when using only NO. The reaction was favoured by the presence of oxygen (not shown). This behaviour was observed for all the clay-based catalysts, reaching conversions close to $80 \%$ for propene and to $40 \%$ for NO. The yield to nitrogen runs parallel to the conversion of NO, no other nitrogenous species were detected. In all catalysts studied, the oxidant contribution is able to produce the complete combustion of the hydrocarbon, without evidences of the presence of $\mathrm{CO}$, behaviour similar to that described for NO reaction.

The effect of the amount of oxygen in the oxidant mixture $\mathrm{NO}+\mathrm{O}_{2}$ was investigated by adding increasing amounts of oxygen under isothermal conditions, at 400 and $500{ }^{\circ} \mathrm{C}$. All the catalysts showed a similar behaviour, shown in Figure 10B. At $400{ }^{\circ} \mathrm{C}$, when the conversion of propene has not reached $100 \%$, the addition of oxygen favoured the oxidation of the hydrocarbon without provoking a decrease in the reduction of the nitrogen oxides, even for high oxygen concentrations. This supposes the existence of a synergic effect between $\mathrm{O}_{2}$, NO and the $\mathrm{O}-\mathrm{Cr}$ species at this temperature. However, at $500{ }^{\circ} \mathrm{C}$, when the conversion of propene was already $100 \%$, the excess of oxygen competed with the nitrogen 
oxides for the oxidation of propene, the hydrocarbon reacted preferably with oxygen strongly decreasing the conversion of the nitrogen oxides. A modified Marsvan Krevelen model has been claimed in the literature for explaining the synergy between $\mathrm{O}_{2}$ and NO for total oxidation abatement of VOC's [58]. The authors proposed that firstly the $\mathrm{NO}$ would react with $\mathrm{O}_{2}$ at the catalyst surface to form $\mathrm{NO}_{2}$. Consequently, the $\mathrm{NO}_{2}$ could assist or replace the oxygen to oxidize the catalyst yielding $\mathrm{MO}_{\mathrm{x}}$ species suitable to oxidize the organic compounds in a final step.
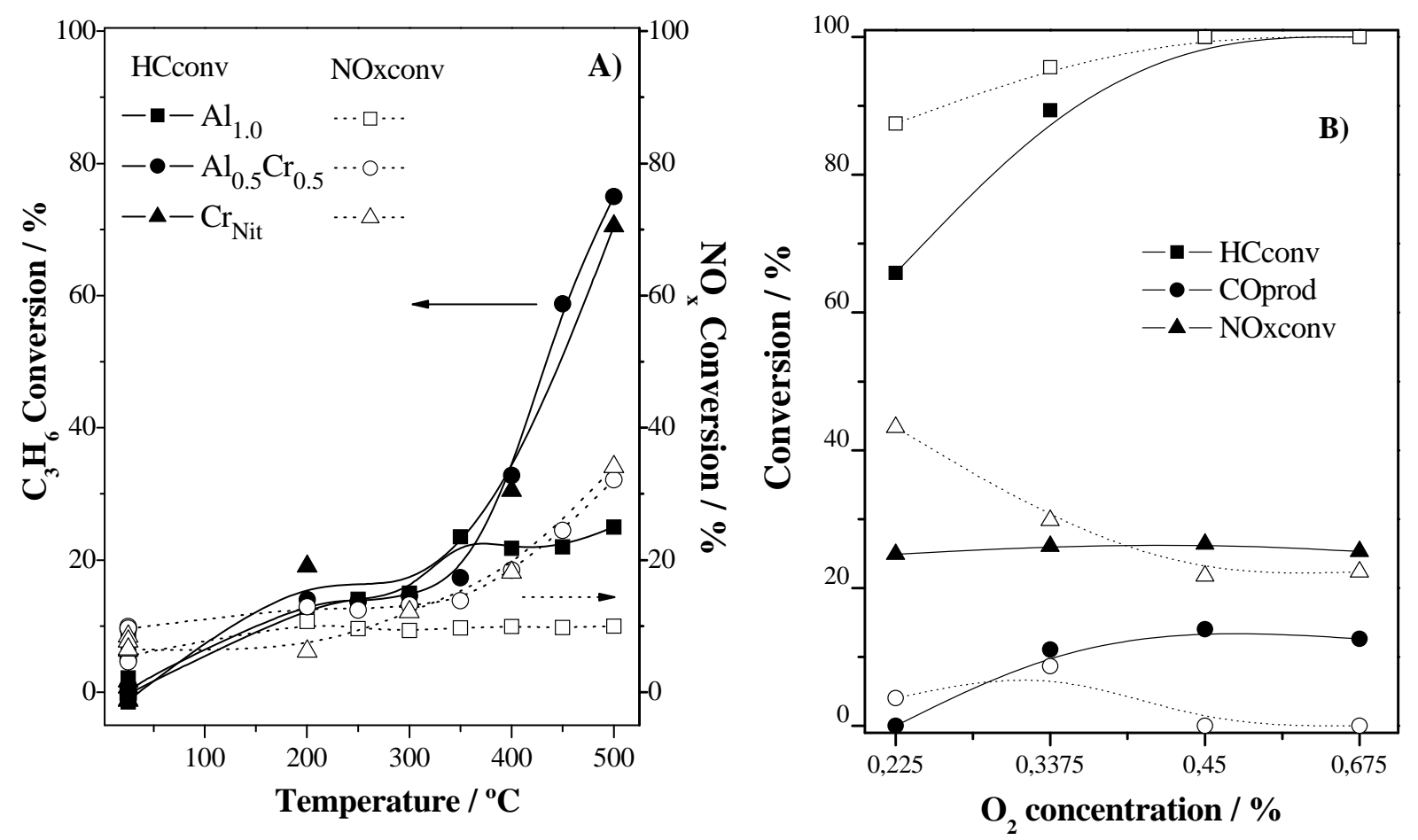

Fig. 10. Catalytic performance results with $\mathrm{NO}$ as oxidant: A) $\mathrm{C}_{3} \mathrm{H}_{6}$ and $\mathrm{NO}_{x}$ conversions for the catalysts indicated, showing the $\mathrm{Al}_{1.0}$ as reference; $\left.\mathrm{B}\right) \mathrm{C}_{3} \mathrm{H}_{6}, \mathrm{NO}_{x}$ conversions and $\mathrm{CO}$ production for the $\mathrm{Al}_{0.5} \mathrm{Cr}_{0.5}$ catalysts with increasing amounts of $\mathrm{O}_{2}$; (full lines, $400{ }^{\circ} \mathrm{C}$, dotted lines, $\left.500^{\circ} \mathrm{C}\right)$.

Coming back to the oxidation of propene with oxygen, the high activity of $\mathrm{Cr}$ saponite samples is accompanied by the occurrence of high amounts of products of incomplete combustion in the outlet stream, as indicated by the $\mathrm{CO}$ presence. This suggests that the oxygen feed will initially contribute to the chromium oxidation to high oxidation state $\left(\mathrm{Cr}^{6+}\right)$ and, then, to be reduced to low oxidation states by the organic compounds and/or the reaction intermediates [53]. The reduction of $\mathrm{Cr}^{3+}$ to lower oxidation species should be rather difficult, as has been indicated before in the $\mathrm{H}_{2}-\mathrm{TPR}$ measurements, requiring high temperatures or a stronger reducing 100 
III. Chromium-saponite clay catalysts: Preparation, characterization and catalytic performance in propene oxidation

agent in the feed. This should limit the activity, justifying in this case the uncompleted degradation of the hydrocarbon.

In order to gain more information on the influence of chromium phases and of the clay support in the redox behaviour of the catalyst, the same reaction was performed using a $\mathrm{Cr} /$ alumina catalyst as reference (not shown), synthesized in the same way that the clay-based impregnated nitrate sample $\left(\mathrm{Cr}_{\text {Nit }}\right)$. This solid shows the same behaviour that the clay-catalysts indicated before, such as $70 \%$ hydrocarbon conversion and $35 \%$ CO selectivity. This fact suggests that the other elements present in the clay, Fe and $\mathrm{Mn}$, are not involved in the oxidation-reduction process, only the chromium species $\left(\mathrm{CrO}_{x}\right)$ incorporated to the clay are responsible to the activity.

\section{II .4. Conclusions}

Treatment of a saponite with polycations of aluminium, chromium or mixtures of the two elements successfully gives rise to intercalated layered solids with high basal spacing. Although the presence of chromium worsen the thermal resistance of the pillared materials, modulating the $\mathrm{Al} / \mathrm{Cr}$ ratio it is possible to obtain solids that adequately combine the stability of alumina-pillared clays and the catalytic ability of chromium. Impregnation of an alumina-pillared saponite support with various chromium-precursors produces, in some cases, the delamination of the layered structure.

The pillared clays and the impregnated materials show high specific surface area and porosity. Their performance for oxidation of propene is very similar independently of the method used for the incorporation of chromium.

\section{III.5. References}

[1] D.E.W. Vaughan, Catal. Today 2 (1988) 187.

[2] K. Othsuka, Chem. Mater. 9 (1997) 2039.

[3] J.-F. Lambert, G. Poncelet, Topics Catal. 4 (1997) 43.

[4] H.G. Karge, J Weitkamp (Eds.), Molecular Sieves - Science and Technology, Volume 1, Synthesis, Springer, Berlin and Heidelberg, 1998.

[5] A. Gil., L.M. Gandía, M.A. Vicente, Catal. Rev.- Sci. Eng. 42 (2000) 145. 
[6] Z. Ding., T.J. Kloprogge, R.L. Frost, G.Q. Lu, H.Y. Zhu, J. Porous Mater. 8 (2001) 273.

[7] F. Wypych, K.G. Satyanarayana (Eds.), Clay Surfaces - Fundamentals and Applications, Elsevier, Amsterdam, 2004.

[8] M. Thompson, R.E. Connick, Inorg. Chem. 20 (1981) 2279.

[9] J.E. Finholt, M.E. Thompson, R.E. Connick, Inorg. Chem. 20 (1981) 4151.

[10] H. Stünzi, W. Marty, Inorg. Chem. 22 (1983) 2145.

[11] L. Spiccia W. Marty, Inorg. Chem. 25 (1986) 266.

[12] L. Spiccia, H. Stoeckli-Evans, W. Marty, R. Giovanoli, Inorg. Chem. 26 (1987) 474.

[13] L. Spiccia, W. Marty, R. Giovanoli, Inorg. Chem. 27 (1988) 2660.

[14] G. Johansson, Acta Chem. Scand. 14 (1960) 771.

[15] N. Lahav, U. Shani, J. Shabtai, Clays Clay Miner. 26 (1978) 107.

[16] J.Y. Bottero, J.M. Cases, F. Fiessinger, J.E. Poirier, J. Phys. Chem. 84 (1980) 2933.

[17] K.A. Carrado, S.L. Suib, N.D. Skoularikis, R.W. Coughlin, Inorg. Chem. 25 (1986) 4217.

[18] D.Y. Zhao, Y.S. Yang, X.X. Guo, Zeolites 15 (1995) 58.

[19] R. Toranzo, M.A. Vicente, M.A. Bañares-Muñoz, Chem. Mater. 9 (1997) 1829.

[20] L. Storaro, R. Ganzerla, M. Lenarda, R. Zanoni, A. Jiménez-López, P. OliveraPastor, E. Rodríguez-Castellón, J. Mol. Catal. A 115 (1997) 329.

[21] P. Olivera-Pastor, J. Maza-Rodríguez, P. Maireles-Torres, E. RodríguezCastellón, A. Jiménez-López, J. Mater. Chem. 4 (1994) 179.

[22] B.M. Weckhuysen, I.E. Wachs, R.A. Schoonheydt, Chem. Rev. 96 (1996) 3327.

[23] A.Z. Abdullah, M.Z. Abu Bakar, S. Bhatia, J. Chem. Technol. Biotechnol. 80 (2005) 1016.

[24] A. Gil, M.A. Vicente, R. Toranzo, M.A. Bañares, L.M. Gandía, J. Chem. Technol. Biotechnol. 72 (1998) 131.

[25] M. Kang, C. Lee, Appl. Catal. A 266 (2004) 163.

[26] V.F. Tret'yakov, T.N. Burdeinaya, Y.P. Zakorchevnaya, A.G. Zakirova, M.N. Bakhtiyarov, V.A. Matyshak, V.N. Korchak, Kinetic. Catal. 46 (2005) 525; T.N. Burdeinaya, V.A. Matyshak, V.F. Tret'yakov, A.G. Zakirova, V.N. Korchak, V.V. Lunin, Kinetic. Catal. 48 (2007) 84.

[27] M.A. Vicente, A. Meyer, E. González, M.A. Bañares-Muñoz, L.M. Gandía, A. Gil, Catal. Lett. 78 (2002) 99. 
[28] G. Ertl, H. Knözinger, J. Weitkamp (Eds.), Handbook of Heterogenous Catalysis. Volume 1, Wiley-VCH, Weinheim, Germany, 1997.

[29] G. Ertl, H. Knözinger, J. Weitkamp (Eds.), Preparation of Solid Catalysts, Wiley-VCH, Weinheim, Germany, 1999.

[30] C.-C. Chien, W.-P. Chuang, T.-J. Huang, Appl. Catal. A 131 (1995) 73.

[31] I.V. Yentekakis, V. Tellou, G. Botzolaki, I.A. Rapakousios, Appl. Catal. B 56 (2005) 59.

[32] G.W. Brindley, S. Yamakana, Am. Miner. 64 (1979) 830.

[33] C. Volzone, Clays Clay Miner. 43 (1995) 377.

[34] S. Chevalier, H. Suquet, R. Franck, C. Marcilly, D. Pp 32-46 in Expanded clays and other microporous solids, M.L. Occelli and H.E. Robson, eds. Van Nostrand Reinhold, New York, 1992.

[35] L. Bergaoui, J.F. Lambert, R. Franck, H. Suquet, J.L. Robert, J. Chem. Soc. Faraday Trans. 91 (1995) 2229.

[36] L. Bergaoui, J.F. Lambert, M.A. Vicente Rodríguez, L.J. Michot, F. Villiéras, Langmuir 11 (1995) 2849.

[37] M.A. Vicente, M. Suárez, J.D. López-González, M.A. Bañares-Muñoz, Langmuir 12 (1996) 566.

[38] O. Prieto, M.A. Vicente, M.A Bañares-Muñoz, J. Porous Mater. 6 (1999) 335.

[39] K.S.W. Sing, D.H. Everett, R.A.W. Haul, L. Moscou, R.A. Pierotti, J. Rouquerol, T. Siemieniewska, Pure Appl. Chem. 57 (1985) 603.

[40] F. Rouquerol, J. Rouquerol, K. Sing, Adsorption by powders and porous solids. Principles, Methodology and Applications, Academic Press, London, 1999.

[41] G. Horvath, K. Kawazoe, J. Chem. Eng. Jpn. 16 (1983) 470.

[42] B. Casal, J. Merino, E. Ruiz-Hitzky, E. Gutiérrez, A. Alvarez, Clay Miner. 32 (1997) 41.

[43] A. Gil, M.A. Vicente, L.M. Gandía, Micropor. Mesopor. Mater. 34 (2000) 115.

[44] M.A. Vicente, R. Toranzo, M.A. Bañares-Muñoz, E. Rodríguez, An. Quím. 94 (1998) 136.

[45] F.M. Labajos, V. Rives, Inorg. Chem. 35 (1996) 5313.

[46] M. del Arco, D. Carriazo, C. Martín, A.M. Pérez-Grueso, V. Rives, J. Solid State Chem. 178 (2005) 3571.

[47] B.M. Weckhuysen, L.M. de Ridder, P.J. Grobet, R.A. Schoonheydt, J. Phys. Chem. B 99 (1995) 390.

[48] Z. Zhu, Z. Chang, L. Kevan, J. Phys. Chem. B 103 (1999) 2680.

[49] K.T. Ranjit, L. Kevan, J. Phys. Chem. B 107 (2003) 2610. 
[50] J.M. Goupil, J.-F. Hemidy, D. Cornet, J. Chim. Phys. 73 (1976) 431.

[51] Z. Zhu, M. Hartmann, E.M. Maes, R.S. Czernuszewicz, L. Kevan, J. Phys. Chem. B 104 (2000) 4690.

[52] C. Belver, M.A. Vicente, A. Martínez-Arias, M. Fernández-García, Appl Catal. B 50 (2004) 227.

[53] J.M. Yáñez-Limón, J.F. Pérez-Roblez, J. González-Hernández, R. ZamoranoUlloa, D. Ramirez-Rosales, Thin Solid Films 373 (2000) 184.

[54] V.R. Choudhary, S. Banerjee, S.G. Pataskar, Appl. Catal. A 253 (2003) 65.

[55] A.Z. Abdullah, M.Z. Abu Bakar, S. Bhatia, J. Hazard. Mater. B 129 (2006) 39.

[56] V.R. Choudhary, G.M. Deshmukh, Chem. Eng. Sci. 60 (2005) 1575.

[57] P. Mars, D.W. van Krevelen, Spec. Sup. Chem. Eng. Sci. 3 (1954) 41.

[58] F. Bertinchamps, M. Treinen, N. Blangenois, E. Mariage and E.M. Gaigneaux, J. Catal. 230 (2005) 493. 


\section{Capítulo I V}

Estudio de la preparación de catalizadores cromo-saponita pilareada y de su comportamiento catalítico en la oxidación de propeno 



\section{Resumen}

Se han preparado catalizadores Cr-saponita por dos métodos de síntesis. Por un lado, intercalación de la saponita con aluminio, con cromo, y conjunta con aluminio y cromo, utilizando diferentes razones molares Al/Cr. Por otro lado, la saponita pilareada con aluminio se usa como soporte para la impregnación con diferentes precursores de cromo con estados de oxidación II, III y VI. Con ello se obtienen sólidos que combinan la naturaleza laminar de la arcilla, la elevada estabilidad térmica aportada por el aluminio y las propiedades catalíticas del cromo. Los catalizadores resultantes son estudiados en la oxidación de propeno mediante oxígeno, óxidos de nitrógeno o mezclas de ambos oxidantes.

\section{Abstract}

Chromium-saponite catalysts have been prepared by two synthesis methods. The first method consists in the intercalation of the saponite with aluminium, with chromium and co-intercalation of $\mathrm{Al}$ and $\mathrm{Cr}$ using several molar ratios of both elements. In the second method, Al-pillared saponite is used as support to be impregnated with various Cr-precursors with different oxidation states. Thus, the solids obtained combine the layered structure of the clay, the high thermal stability given by the Al-pillars and the catalytic activity given by $\mathrm{Cr}$. These catalysts have been studied in the oxidation of propene with oxygen, nitrogen oxides and mixtures of both oxidants.

\section{V.1. I ntroducción}

Las arcillas pilareadas son uno de los grupos de sólidos más estudiados en los últimos años ya que constituyen una de las familias más importantes de sólidos microporosos desarrollados por ingeniería molecular. El proceso de síntesis puede dividirse en varios pasos: (1) preparación de polihidroxicationes metálicos mediante hidrólisis de las sales correspondientes, (2) intercambio iónico de los cationes inicialmente presentes en el espacio interlaminar por los policationes previamente sintetizados, formando así los sólidos intercalados, y (3) estabilización de los sólidos intercalados mediante calcinación, obteniendo así los sólidos pilareados. El proceso de intercalación produce un importante incremento del espaciado basal de 
la arcilla de partida mientras que el proceso de calcinación da lugar a la deshidratación y deshidroxilación de los policationes intercalados formando clusters de los óxidos metálicos correspondientes. Los clusters metálicos, también llamados pilares, mantienen las láminas separadas y permiten el acceso a la superficie laminar interna generando así un sólido con estructura microporosa de dimensiones moleculares. Desde otro punto de vista, se puede considerar que las láminas impiden la agregación de las especies metálicas intercaladas, produciendo una elevada dispersión de las especies intercalantes. El tamaño y número de los oligómeros intercalados determina el tamaño de los poros de la arcilla pilareada [1].

La intercalación de policationes de cromo resulta especialmente interesante debido a las propiedades catalíticas de este elemento que, combinadas con las superficiales de las arcillas, pueden dar lugar a sólidos finales con gran diversidad de aplicaciones. La cointercalación con disoluciones mixtas de aluminio y cromo se lleva a cabo mediante la hidrólisis conjunta de ambos cationes variando la razón molar $\mathrm{OH}^{-} / \mathrm{Me}^{3+}$ en función de la cantidad de cromo presente en la disolución intercalante. El cromo se incorpora a la estructura de la arcilla aunque no está claro si ambos elementos forman policationes independientes o si el cromo entra a formar parte del policatión de Keggin mediante sustitución isomórfica del aluminio, como han descrito algunos autores [2].

Por otro lado, los catalizadores soportados están formados por una o más sustancias catalíticamente activas depositadas sobre la superficie de un soporte con elevada superficie específica. En ellos, se trata de conseguir la dispersión de los compuestos activos y evitar la sinterización. La preparación de catalizadores soportados tiene lugar en varias etapas que incluyen el depósito del precursor activo sobre la superficie del soporte, secado, calcinación y, en algunos casos, una última etapa de activación. El uso de catalizadores soportados responde a la necesidad de maximizar la eficacia disminuyendo los costes. Con este tipo de catalizadores se necesita una menor cantidad de fase activa con una elevada dispersión, y se facilita la manejabilidad del catalizador.

Por otra parte, catalizadores basados en cromo [3] han sido ampliamente utilizados en algunas reacciones de interés como oxidación de hidrocarburos [4], VOCs [5] y CFCs [6], sin embargo en pocos casos se trata de sólidos arcillosos [7]. 
En todo caso, los diferentes estados de oxidación del cromo aportan un gran número de posibilidades en reacciones redox [8].

En este trabajo se describe la preparación, caracterización y comportamiento catalítico de varios catalizadores cromo-saponita pilareada. Los catalizadores han sido preparados por diferentes métodos: (1) intercalación y pilarización con policationes de aluminio, cromo y diferentes razones molares $\mathrm{Al} / \mathrm{Cr}, \mathrm{y}$ (2) impregnación de un soporte de arcilla pilareada con aluminio con diferentes precursores de cromo que contienen el metal en tres estados de oxidación diferentes y con distintos contraiones.

\section{IV.2. Experimental}

La arcilla utilizada como material de partida es una saponita procedente del yacimiento de Yunclillos (Toledo), que es suministrada por la empresa TOLSA S.A. (Madrid). Experimentalmente se utiliza la fracción menor de dos micras que se obtiene mediante suspensión y decantación en agua. La fórmula estructural por celda unidad es: $\left[\mathrm{Si}_{7,348} \mathrm{Al}_{0,652}\right]\left[\mathrm{Al}_{0,178} \mathrm{Mg}_{5,369} \mathrm{Mn}_{0,004} \mathrm{Fe}_{0,154} \mathrm{Ti}_{0,022}\right] \mathrm{O}_{20}(\mathrm{OH})_{4}\left[\mathrm{Ca}_{0,096}\right.$ $\left.\mathrm{Na}_{0,066} \mathrm{Mg}_{0.230} \mathrm{~K}_{0,081}\right]$. Su capacidad de cambio catiónico es 0,99 meq/g y su espaciado basal es $13,8 \AA$.

Esta saponita ha sido intercalada con cromo, con aluminio y con disoluciones mixtas de ambos elementos. La intercalación con policationes de cromo se lleva a cabo mediante un método descrito en bibliografía $[9,10]$. Se utiliza la cantidad de sal necesaria para conseguir 30 mmoles de metal, que se disuelven en agua y se hidrolizan con una disolución de $\mathrm{NaOH}$ de manera que la razón molar $\mathrm{OH}^{-} / \mathrm{Cr}^{3+}$ sea 2,0 . La disolución obtenida se envejece durante 72 horas para facilitar la formación de policationes. Transcurrido ese tiempo la disolución se añade sobre una suspensión de 6 gramos de arcilla y se mantienen en contacto durante otras 72 horas, tiempo en el que tiene lugar el proceso de intercambio iónico. La suspensión resultante se centrifuga y se lava mediante diálisis hasta ausencia de cloruros, utilizando nitrato de plata para el análisis. El sólido obtenido se seca a $70{ }^{\circ} \mathrm{C}$ durante 24 horas y se calcina utilizando una rampa de temperatura de $1{ }^{\circ} \mathrm{C} / \mathrm{min}$ hasta alcanzar los $500{ }^{\circ} \mathrm{C}$, temperatura a la que se mantiene durante 2 horas, obteniéndose así el sólido pilareado. 
La intercalación con aluminio se realiza mediante un método estandarizado [11]. La disolución de $\mathrm{AlCl}_{3} \cdot 6 \mathrm{H}_{2} \mathrm{O}$ se hidroliza con una disolución de $\mathrm{NaOH}$ de manera que la relación $\mathrm{OH}^{-} / \mathrm{Al}^{3+}$ sea igual a 2,2. Una vez terminada la adición la disolución se deja envejecer durante 24 horas. La disolución se añade a una suspensión previamente preparada de arcilla en agua utilizando una razón Al/arcilla de $5 \mathrm{mmoles} / \mathrm{g}$ y se mantienen en contacto 24 horas para favorecer el intercambio iónico. Para la obtención del sólido intercalado la arcilla se centrifuga y se lava mediante diálisis hasta ausencia de cloruros. El sólido pilareado se obtiene tras secado y calcinación en las condiciones descritas anteriormente.

Además, se lleva a cabo la intercalación conjunta de policationes de aluminio y cromo. Las disoluciones intercalantes contienen cantidades diferentes de ambos metales en razones molares $\mathrm{Al} / \mathrm{Cr}$ que son $0,9: 0,1 ; 0,5: 0,5$ y 0,2:0,8. Estas disoluciones se hidrolizan con $\mathrm{NaOH}$ utilizando razones molares $\mathrm{OH}^{-} / \mathrm{Me}^{3+}$ que están comprendidas entre 2,2 y 2,0 en función del contenido en cromo de cada una de ellas. Tanto el tiempo de envejecimiento de las disoluciones de policationes como el tiempo de contacto policatión-arcilla están comprendidas entre 24 y 72 horas, aumentando con la cantidad de cromo en la disolución intercalante. El resto de las condiciones de síntesis son modificaciones de las descritas para la preparación de policationes de aluminio o de cromo, aproximándose a unas o a otras según la composición de la disolución $[9,11]$. Una vez producido el intercambio iónico los sólidos se centrifugan y se lavan mediante diálisis hasta ausencia de cloruros, tras lo cual se secan y se calcinan de la misma forma que en los casos anteriores.

Los sólidos soportados se obtienen mediante impregnación de la saponita pilareada con Al por el método de humedad incipiente. Como fase activa se utilizan diferentes precursores que contienen cromo: acetato de cromo(II), acetilacetonato de cromo(III), cromato amónico y nitrato de cromo(III). De esta forma se pueden comparar los resultados obtenidos en función del estado de oxidación del cromo y del disolvente utilizado, ya que el acetilacetonato de cromo(III) fue impregnado utilizando acetona como disolvente mientras que los demás precursores fueron disueltos en agua. La cantidad de precursor utilizada en cada caso fue la necesaria para obtener un porcentaje del $8 \%$ en peso de $\mathrm{Cr}_{2} \mathrm{O}_{3}$ en los sólidos finales. Para realizar la impregnación los precursores se disuelven en la mínima cantidad de disolvente necesaria para la disolución completa del precursor siendo posible realizar todas las impregnaciones en una etapa. Una vez terminada la 
impregnación, los sólidos se secan a temperatura ambiente y se calcinan en las mismas condiciones que los sólidos pilareados y copilareados. Además de los sólidos impregnados sobre un soporte arcilloso, se realiza una impregnación de nitrato de cromo(III) sobre alúmina $\left(\mathrm{Y}-\mathrm{Al}_{2} \mathrm{O}_{3}\right.$ Condea de Puralox), que puede ser considerada como soporte de referencia al ser ampliamente empleada en reacciones de oxidación [12].

La nomenclatura de los sólidos preparados es la siguiente: para los sólidos preparados mediante intercalación se utiliza la nomenclatura $\mathrm{Al}_{x} \mathrm{Cr}_{\mathrm{y}}$ siendo $\mathrm{x}$ e y las correspondientes razones molares de cada metal utilizadas en la intercalación. Una vez calcinados se añade "-500" al nombre. Para los sólidos impregnados se utiliza la nomenclatura $\mathrm{Cr}_{\mathrm{Ac}}, \mathrm{Cr}_{\text {acac, }}$ Cromato y $\mathrm{Cr}_{\text {Nit }}$ según los precursores utilizados: acetato de cromo(II), acetilacetonato de cromo(III), cromato amónico y nitrato de cromo(III), respectivamente. El sólido preparado por impregnación de nitrato de cromo(III) sobre alúmina se designa Cr/alúmina. Una vez calcinados también se añade "-500" a la nomenclatura.

\section{IV.3. Técnicas de caracterización}

El análisis químico elemental de las muestras fue realizado mediante espectroscopía de plasma acoplado inductivamente (ICPS). Los resultados obtenidos se utilizan para calcular la fórmula elemental de la saponita de partida, así como para determinar la cantidad de cromo y de aluminio que se introduce en cada síntesis, y las modificaciones que éstas producen en la composición química de la saponita de partida.

La difracción de rayos $X$ de las muestras en polvo se realiza con un difractómetro Siemens D-500 con intensidad y voltaje de $30 \mathrm{~mA}$ y $40 \mathrm{kV}$, respectivamente. El equipo utiliza la radiación $\mathrm{Ka}$ del cobre filtrada. El barrido de los sólidos se realizó entre 2 y $65^{\circ}$ de $2 \theta$ sobre muestras de polvo no orientado, con una velocidad de barrido de $2^{\circ}$ de ángulos de Bragg $(2 \theta)$ por minuto.

El estudio textural de las muestras se llevó a cabo mediante la realización de las isotermas de adsorción-desorción de nitrógeno a -196 C, que son obtenidas en un analizador Micromeritics ASAP 2010. Antes de llevar a cabo el estudio de adsorción las muestras son desgasificadas durante dos horas a temperatura ambiente y otras 
dos horas a $110{ }^{\circ} \mathrm{C}$ o hasta alcanzar una presión inferior a $50 \mu \mathrm{m} \mathrm{Hg}$. De estas isotermas se obtiene la superficie específica, utilizando el método BET, y la superficie externa mediante aplicación del método $t$.

Los ensayos de actividad se llevaron a cabo en un reactor de flujo de lecho fijo. El lecho catalítico está constituido por el catalizador previamente tamizado a un tamaño de grano en el rango de 0,125-0,250 mm. Dicho tamaño fue optimizado con objeto de eliminar limitaciones a la velocidad de reacción debidas a efectos de transferencia de masa. Para todos los catalizadores se analizó la conversión en una mezcla estequiométrica $0,1 \% \mathrm{C}_{3} \mathrm{H}_{6}+0,9 \% \mathrm{Ox}$ (oxidante) en $\mathrm{Ar}$, empleando como oxidantes $\mathrm{O}_{2}, \mathrm{NO}_{x}$ o mezcla $\mathrm{O}_{2} / \mathrm{NO}$, a una velocidad espacial de $19028 \mathrm{~h}^{-1}$.

El estudio catalítico fue realizado en condiciones estacionarias desde 200 hasta $500{ }^{\circ} \mathrm{C}$ registrando datos en intervalos de $50{ }^{\circ} \mathrm{C}$. La rampa de calentamiento empleada para cada intervalo fue de $5^{\circ} \mathrm{C} / \mathrm{min}$. Los reactantes y productos fueron analizados en línea con un espectrómetro FTIR Perkin-Elmer 1725X acoplado con una celda de gases en modo transmisión con múltiple reflexión. El análisis de cada gas se desarrolló con un programa diseñado para calcular la concentración de cada especie a partir de la absorbancia de sus bandas características [12].

\section{V.4. Caracterización de los sólidos}

\section{V.4.1. Sólidos intercalados y pilareados}

El sólido intercalado únicamente con policationes de aluminio muestra un difractograma de rayos $X$ con las características propias de las arcillas intercaladas. El espaciado basal se desplaza desde los $13,81 \AA$ de la saponita natural hasta $18,33 \AA$ en el sólido intercalado. La intercalación con policationes mixtos $\mathrm{Al} / \mathrm{Cr}$ también da lugar a un desplazamiento en la reflexión (001) hacia menores ángulos, lo que implica un aumento en el espaciado basal en todas las muestras preparadas. Se puede deducir que la intercalación se produce correctamente en todos los casos estudiados. Una vez terminado el tratamiento térmico se realizan de nuevo los difractogramas de rayos $X$ encontrándose notables diferencias en función del contenido en cromo de las muestras (véase la figura 1 ). 


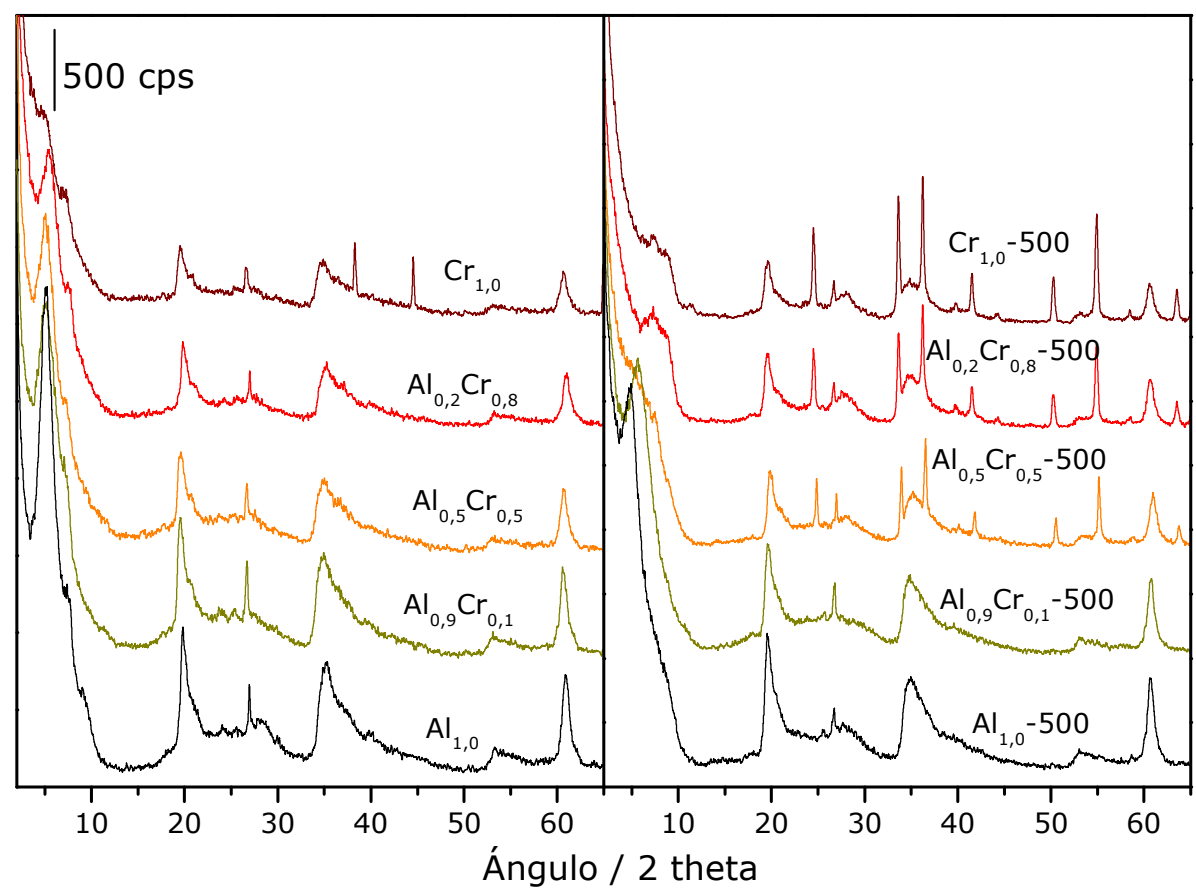

Figura 1: Difractogramas de rayos $X$ de las muestras intercaladas (izquierda) y pilareadas (derecha).

Como se observa en la figura, se produce la intercalación en todas las relaciones $\mathrm{Al} / \mathrm{Cr}$ estudiadas. Tras la calcinación se observa un colapso parcial de la estructura laminar que es más acusado cuanto mayor es el contenido en cromo de las muestras, lo que refleja la menor estabilidad de los policationes de cromo en comparación con los de aluminio. Ninguno de los picos observados se corresponde con óxidos u oxihidróxidos de cromo, lo que permite afirmar que se han obtenido muestras con un elevado grado de dispersión de cromo.

El análisis elemental de las muestras preparadas indica la cantidad de aluminio y cromo fijados durante la intercalación, así como las variaciones en los demás elementos (tabla 1). Los resultados de los análisis, expresados en porcentaje en óxidos, se refieren a muestra seca, para facilitar la comparación de los resultados. Del mismo modo, el contenido de cada óxido metálico se normaliza con respecto al contenido en sílice de la saponita natural. En estos análisis se observan las variaciones en el contenido de aluminio y cromo en función de la disolución utilizada para realizar la intercalación. La cantidad de cromo fijado, expresado como óxido de cromo, oscila entre el 3 y el $25 \%$, lo que indica que mediante éste método de síntesis se puede controlar la cantidad de metal que se incorpora en las muestras. Las relaciones atómicas $\mathrm{Al}: \mathrm{Cr}$ experimentales en los sólidos pilareados con disoluciones mixtas son $5,85: 1,0,80: 1$, y $2: 6,96$ frente a los valores 9:1, 1:1, y $2: 8$, respectivamente, existentes en las disoluciones. Estudiando las variaciones 
de los demás elementos, se observa que el intercambio catiónico se produce principalmente por cationes $\mathrm{Ca}^{2+}$ y $\mathrm{Na}^{+}$presentes en la interlámina de la saponita natural.

\begin{tabular}{ccccccccccc}
\hline Muestra & $\mathrm{SiO}_{2}$ & $\mathrm{Al}_{2} \mathrm{O}_{3}$ & $\mathrm{Fe}_{2} \mathrm{O}_{3}$ & $\mathrm{MnO}$ & $\mathrm{MgO}$ & $\mathrm{CaO}$ & $\mathrm{Na}_{2} \mathrm{O}$ & $\mathrm{K}_{2} \mathrm{O}$ & $\mathrm{TiO}_{2}$ & $\mathrm{Cr}_{2} \mathrm{O}_{3}$ \\
\hline Saponita & 60,09 & 5,77 & 1,65 & 0,03 & 30,70 & 0,71 & 0,27 & 0,52 & 0,25 & 0,00 \\
$\mathrm{Al}_{1,0}$ & 60,09 & 15,50 & 1,61 & 0,03 & 28,35 & 0,12 & 0,04 & 0,47 & 0,24 & 0,00 \\
$\mathrm{Al}_{0,9} \mathrm{Cr}_{0,1}$ & 60,09 & 16,80 & 1,61 & 0,03 & 28,45 & 0,05 & 0,05 & 0,41 & 0,25 & 2,81 \\
$\mathrm{Al}_{0,5} \mathrm{Cr}_{0,5}$ & 60,09 & 12,23 & 1,62 & 0,03 & 27,76 & 0,04 & 0,04 & 0,51 & 0,25 & 12,10 \\
$\mathrm{Al}_{0,2} \mathrm{Cr}_{0,8}$ & 60,09 & 9,16 & 1,61 & 0,03 & 27,86 & 0,04 & 0,03 & 0,42 & 0,24 & 17,50 \\
$\mathrm{Cr}_{1,0}$ & 60,09 & 6,12 & 1,71 & 0,03 & 26,66 & 0,05 & 0,02 & 0,50 & 0,27 & 24,99 \\
\hline
\end{tabular}

Tabla 1. Composición química (\% en peso) de la saponita de Yunclillos y de los sólidos intercalados, normalizada respecto al contenido en $\mathrm{SiO}_{2}$ de la saponita natural.

El estudio de las propiedades texturales de los sólidos refleja la elevada superficie específica que se desarrolla mediante el proceso de intercalación. Los valores de superficie específica estimados por el método BET para los sólidos intercalados y pilareados se presentan en la tabla 2.

\begin{tabular}{|c|c|c|}
\hline Muestra & $\mathrm{S}_{\mathrm{BET}}$ & $\mathrm{S}_{\mathrm{MP}}$ \\
\hline Saponita & 169 & $111(66) *$ \\
$\mathrm{Al}_{1,0}$ & 334 & $283(85)$ \\
$\mathrm{Al}_{0,9} \mathrm{Cr}_{0,1}$ & 258 & $217(84)$ \\
$\mathrm{Al}_{0,5} \mathrm{Cr}_{0,5}$ & 311 & $270(87)$ \\
$\mathrm{Al}_{0,2} \mathrm{Cr}_{0,8}$ & 327 & $285(87)$ \\
$\mathrm{Cr}_{1,0}$ & 328 & $280(85)$ \\
$\mathrm{Al}_{1,0}-500$ & 274 & $218(80)$ \\
$\mathrm{Al}_{0,9} \mathrm{Cr}_{0,1}-500$ & 240 & $191(80)$ \\
$\mathrm{Al}_{0,5} \mathrm{Cr}_{0,5}-500$ & 252 & $207(82)$ \\
$\mathrm{Al}_{0,2} \mathrm{Cr}_{0,8}-500$ & 191 & $120(63)$ \\
$\mathrm{Cr}_{1,0}-500$ & 209 & $117(56)$ \\
\hline
\end{tabular}

Tabla 2. Superficie específica de los sólidos $\left(\mathrm{S}_{\mathrm{BET}}\right)$ y superficie de microporos de cada una de ellas $\left(S_{M P}\right)$. * Entre paréntesis, porcentaje de superficie total que corresponde a la superficie de los microporos.
Como se observa en los datos de la tabla el proceso de intercalación incrementa la superficie de los sólidos, mientras que el tratamiento térmico y consiguiente deshidratación y deshidroxilación de los pilares produce una disminución de la superficie específica respecto a los intercalados. A partir de la superficie externa se evalúa la superficie microporosa, cuyos valores y sus porcentajes respecto a la superficie total se ofrecen también en la Tabla. La superficie microporosa contribuye de forma muy significativa a la superficie total de los sólidos, alcanzando valores entre el 84 y el $87 \%$ en los sólidos intercalados y entre el 56 y el $82 \%$ en los sólidos pilareados. La disminución de la superficie se debe al colapso de la estructura laminar observado en los difractogramas de rayos $X$. 


\section{IV.4.2. Sólidos impregnados}

Los sólidos impregnados se caracterizan utilizando las mismas técnicas que en el caso de los intercalados y pilareados. Los difractogramas de las muestras impregnadas se comparan con el del sólido $\mathrm{Al}_{1,0}-500$, utilizado como soporte y se realizan también los difractogramas después de la calcinación, todos ellos se presentan en la figura 2.

El proceso de impregnación mantiene en todas las muestras la estructura laminar del sólido $\mathrm{Al}_{1,0}-500$ utilizado como soporte. Se mantienen todos las líneas de difracción del silicato $y$, en algunas muestras, aparecen picos característicos de las sales precursoras. La disminución de intensidad de los picos indica que, aunque se mantiene la estructura de la arcilla, el proceso de impregnación disminuye notablemente la cristalinidad del sólido pilareado utilizado como soporte. Los difractogramas de los sólidos una vez calcinados no muestran los picos de los precursores y el pico a bajos ángulos varía considerablemente indicando un colapso parcial de la estructura laminar. En ninguno de los sólidos se observan reflexiones propias de óxidos u oxihidróxidos de cromo. Esto indica que durante la síntesis de los sólidos impregnados no se forman especies cristalinas de los mismos, detectables mediante esta técnica, lo que hace suponer que el cromo incorporado tiene una buena dispersión sobre el soporte.

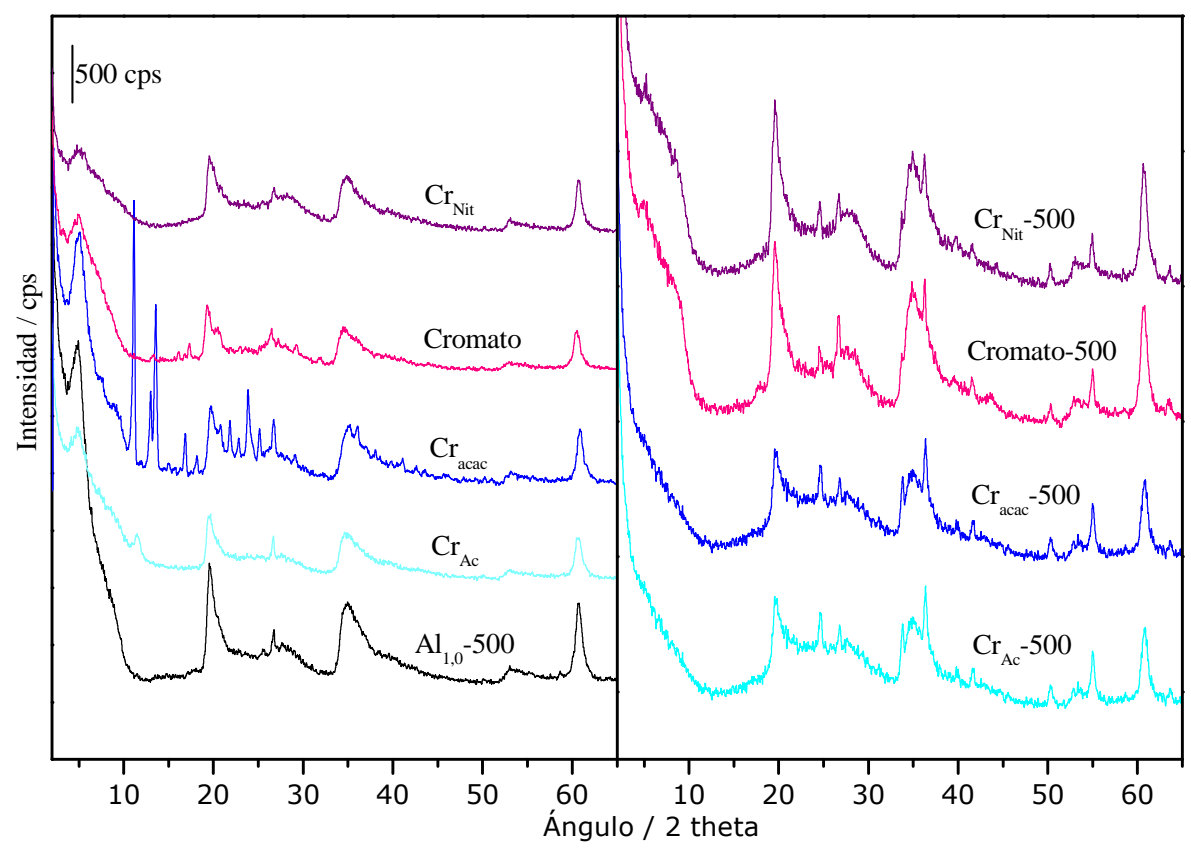

Figura 2. Difractogramas de rayos $X$ de las muestras impregnadas. Izqda: muestras impregnadas y secas a $70^{\circ} \mathrm{C}$, se incluye el soporte. Dcha: Muestras impregnadas calcinadas. 


\begin{tabular}{|c|c|}
\hline Muestra & $\mathrm{Cr}_{2} \mathrm{O}_{3} / \%$ \\
\hline $\mathrm{Al}_{1,0}$ & 0,00 \\
$\mathrm{Cr}_{\mathrm{Ac}}$ & 9,17 \\
$\mathrm{Cr}_{\text {acac }}$ & 8,08 \\
Cromato & 8,93 \\
$\mathrm{Cr}_{\text {Nit }}$ & 8,51 \\
\hline
\end{tabular}

Tabla 3. Porcentaje de cromo en el soporte $y$ en las muestras impregnadas.

\begin{tabular}{|c|c|}
\hline Muestra & $\mathrm{S}_{\mathrm{BET}} / \mathrm{m}^{2} \mathrm{~g}^{-1}$ \\
\hline $\mathrm{Al}_{1,0}-500$ & 274 \\
\hline $\mathrm{Cr}_{\mathrm{Ac}}$ & 178 \\
\hline $\mathrm{Cr}_{\text {acac }}$ & 22 \\
\hline Cromato & 72 \\
\hline $\mathrm{Cr}_{\text {Nit }}$ & 6 \\
\hline $\mathrm{Cr}_{A c}-500$ & 177 \\
\hline $\mathrm{Cr}_{\mathrm{acac}}-500$ & 237 \\
\hline Cromato-500 & 97 \\
\hline $\mathrm{Cr}_{\mathrm{Nit}}-500$ & 107 \\
\hline
\end{tabular}

Las muestras impregnadas se preparan de forma que el sólido final contenga un $8 \%$ en peso de $\mathrm{Cr}_{2} \mathrm{O}_{3}$. Los análisis químicos de las muestras dan los contenidos en cromo incluidos en la tabla 3. Los porcentajes de los demás óxidos se mantienen prácticamente constantes, ya que la impregnación no produce modificación química alguna en el soporte.

La superficie específica de los sólidos calculada a partir de los resultados de adsorción de nitrógeno a $-196{ }^{\circ} \mathrm{C}$ da resultados muy diferentes para los sólidos impregnados secos y los calcinados. Los sólidos impregnados secos a $70{ }^{\circ} \mathrm{C}$ poseen superficies mucho menores que la del soporte utilizado para realizar la impregnación. Esto es debido a que durante la impregnación las sales precursoras se sitúan a la entrada de los poros, bloqueando el acceso a los mismos y disminuyendo en gran medida la superficie específica del sólido utilizado como soporte. Durante el proceso de calcinación, como se comprueba con los análisis FTIR y térmicos, las sales utilizadas como precursores de la impregnación pierden los ligandos y contraiones, que constituyen gran parte de su volumen, de manera que se libera de nuevo la entrada a la porosidad $y$, por lo tanto, la superficie específica de los catalizadores aumenta. Como se ha visto en los difractogramas de rayos $X$, la segunda calcinación produce un colapso parcial de la estructura laminar por lo que los catalizadores finalmente obtenidos no alcanzan en ningún caso la superficie del soporte $\mathrm{Al}_{1,0}-500$. 


\section{IV.5. Actividad catalítica}

Los sólidos preparados son ensayados como catalizadores en la oxidación de propeno con distintas mezclas oxidantes. La primera reacción estudiada consiste en la oxidación de hidrocarburo con oxígeno en condiciones estequiométricas, cuyos resultados se muestran en la figura 3 para la conversión del hidrocarburo.

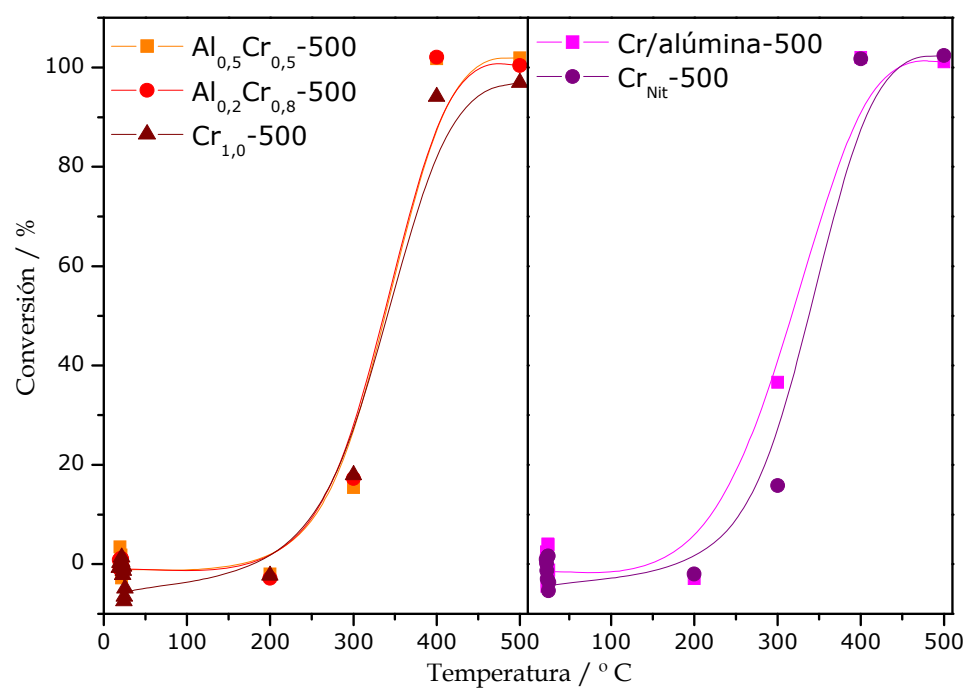

Figura 3. Conversiones de hidrocarburo para la reacción $\mathrm{C}_{3} \mathrm{H}_{6}+\mathrm{O}_{2}$ para los catalizadores indicados: izada) sólidos pilareados; dcha. ) sólidos

Las conversiones de hidrocarburo registradas por los catalizadores pilareados alcanzan el $100 \%$ para temperaturas superiores a los $400{ }^{\circ} \mathrm{C}$, independientemente del contenido en cromo de dichos sólidos. Este efecto refleja la existencia de un porcentaje de cromo, incorporado en los pilares, óptimo para esta reacción, cercano al 10-12 \% en peso. Comparando los resultados de los sólidos intercalados con los preparados por impregnación se observa el mismo comportamiento para todos ellos independientemente de la forma utilizada para la incorporación del cromo, lo que reflejaría la eficacia del comportamiento redox del cromo ante procesos de oxidación, ya se encuentre localizado en la superficie interna del catalizador (sólidos pilareados) o en la superficie externa (sólidos impregnados). El análisis de los efluentes de reacción muestra, sin embargo, la combustión parcial del hidrocarburo para todos los casos a temperaturas superiores a los $350^{\circ} \mathrm{C}$. Como se muestra en la Figura 4 la oxidación del hidrocarburo produce $\mathrm{CO}_{2}$ y $\mathrm{CO}$, alcanzando una producción de CO cercana al $20 \%$. 


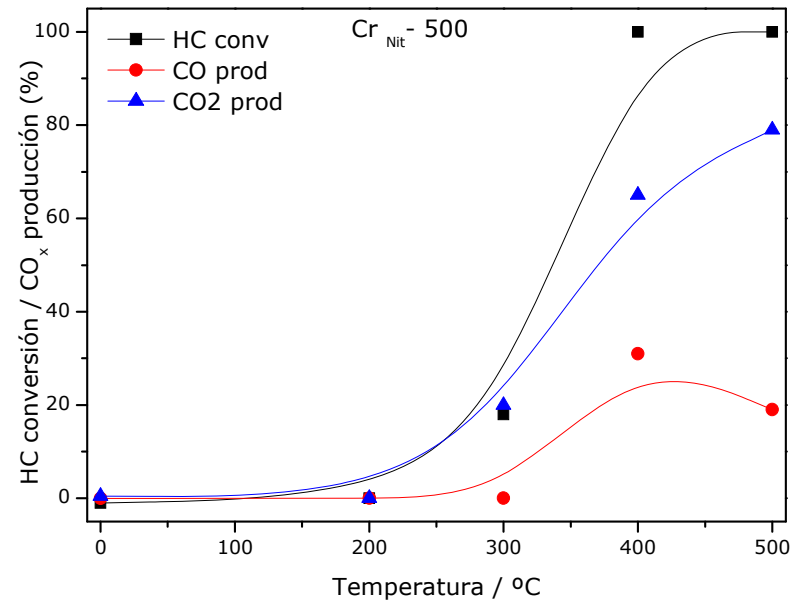

Figura 4. Conversión de hidrocarburo y producción de $\mathrm{CO}_{\mathrm{x}}$ para el sólido impregnado $\mathrm{Cr}_{\mathrm{Nit}}-500$ durante la reacción $\mathrm{C}_{3} \mathrm{H}_{6}+\mathrm{O}_{2}$

En segundo lugar, se estudia como reacción modelo la oxidación del hidrocarburo en presencia de NO en condiciones estequiométricas. En este caso la reacción conlleva un interés medioambiental añadido al estudiar tanto la oxidación del propeno como la reducción del NO [13]. Los resultados obtenidos se muestran en la figura 5, analizando las conversiones del propeno, de los óxidos de nitrógeno, así como el rendimiento de los mismos a nitrógeno diatómico.

La oxidación del hidrocarburo alcanza conversiones cercanas al $60 \%$, a altas temperaturas, tanto en los materiales pilareados con cromo como en los impregnados, lo que difiere considerablemente de las altas conversiones obtenidas al emplear oxígeno como agente oxidante. Al analizar los resultados obtenidos para las conversiones de los óxidos de nitrógeno se observan valores considerablemente bajos, cercanos al $30 \%$. La diferencia existente entre las conversiones obtenidas para oxidante y reductor merece ser objeto de estudio. Dado que no se introduce ningún otro agente oxidante en la alimentación del reactor la oxidación del hidrocarburo debe producirse por un mecanismo tipo Mars-Van Krevelen, por el cual el catalizador es capaz de ceder parte del oxígeno de su estructura para producir la oxidación y regenerarse posteriormente mediante una corriente de oxígeno. Este efecto también es observado en el sólido Cr/alúmina, aunque en menor medida, lo que supondría que el aporte de oxígeno se produce por las especies de cromo formadas tras la calcinación de los materiales a $500{ }^{\circ} \mathrm{C}$, descartando el material arcilloso como fuente del oxidante. 


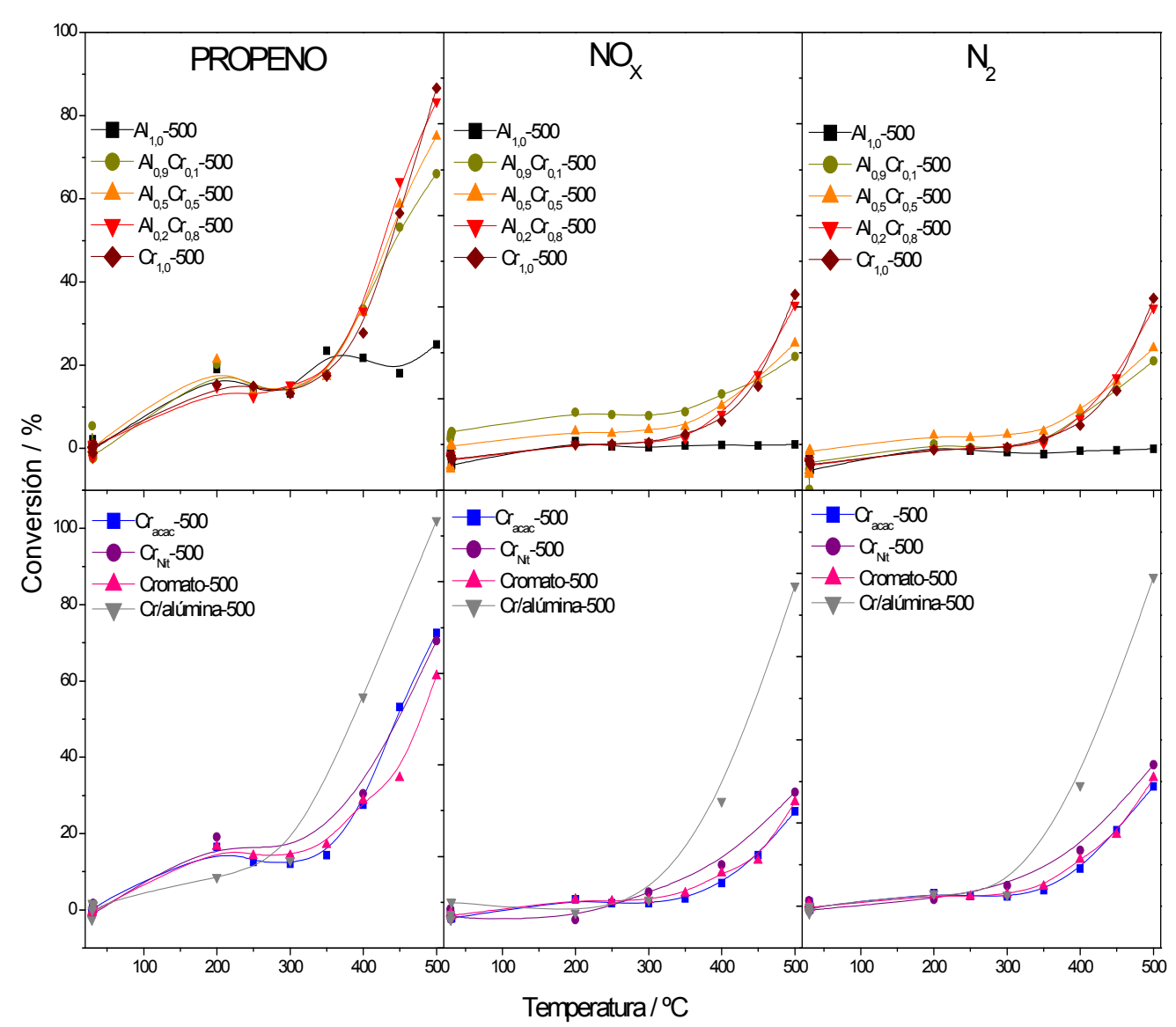

Figura 5. Conversiones de hidrocarburo y $\mathrm{NO}_{\mathrm{x}}$ obtenidas en la reacción y rendimiento a nitrógeno de cada una de: las muestras pilareadas (arriba) y las muestras impregnadas (abajo).

El análisis detallado de los efluentes de esta reacción no muestra evidencias de la oxidación parcial del propeno, a diferencia de lo que ocurría en la primera reacción, observándose sólo la presencia de $\mathrm{CO}_{2}$ como producto. En lo que respecta a los productos nitrogenados, la similitud del rendimiento a nitrógeno con respecto a la reducción de los óxidos de nitrógeno (figura 5) muestra al nitrógeno diatómico como único producto, no detectándose en ningún caso especies de reducción intermedias.

En el caso de las muestras pilareadas se observa que introduciendo pequeñas cantidades de cromo a la saponita es posible alcanzar conversiones notables, sin embargo, a partir de un cierto contenido todas tienen un comportamiento muy similar, independientemente de la cantidad de cromo introducida en el proceso de intercalación. Esta similitud supone, como ocurría en la oxidación con oxígeno, la existencia de un contenido en cromo óptimo o idóneo para esta reacción, que podríamos fijar en torno al $5 \%$ en peso, puesto que para porcentajes superiores no 
se observan variaciones significativas en la actividad de los catalizadores, efecto observado en otros materiales pilareados [14].

Respecto a las muestras impregnadas sobre la saponita pilareada se observa gran similitud entre ellas. El precursor de cromo empleado para la impregnación no parece influir en la actividad del catalizador resultante, ni siquiera en lo que respecta al aporte de oxígeno por parte del catalizador. Este hecho supone que a pesar de partir de precursores con diferentes estados de oxidación del cromo el tratamiento de calcinación, al que los materiales son sometidos, da lugar a sólidos con las mismas especies de cromo que aportan el oxígeno para el mecanismo MarsVan Krevelen. La influencia del soporte en la reacción de propeno con los $\mathrm{NO}_{\mathrm{x}}$ se hace evidente al comparar los sólidos $\mathrm{Cr}$ /alúmina y $\mathrm{Cr}_{\text {Nit }}$. El catalizador basado en alúmina alcanza conversiones, tanto de hidrocarburo como de óxidos de nitrógeno, notablemente superiores a los valores alcanzados por el sólido basado en la saponita pilareada. Puesto que el precursor de cromo empleado para la impregnación fue el mismo en ambos casos y el contenido en cromo es similar, la diferencia de actividad puede ser atribuida a las propiedades superficiales del catalizador.

Dado el distinto comportamiento catalítico que poseen los sólidos sintetizados en función de la naturaleza del agente oxidante, se llevó a cabo la oxidación del propeno en presencia de ambos oxidantes. Para ello se regularon las concentraciones de los reactantes, manteniendo la estequiometría de la reacción y ajustando las proporciones de $\mathrm{NO}$ y de $\mathrm{O}_{2}$ de forma que ambos oxidantes aporten el mismo número de átomos de oxígeno a la reacción $\left(\mathrm{C}_{3} \mathrm{H}_{6}+4,5 \mathrm{NO}+2,25 \mathrm{O}_{2}\right)$. Los resultados obtenidos se muestran en la figura 6 .

Se observa que las conversiones obtenidas utilizando mezcla de oxidantes son mayores que las obtenidas en presencia de NO como único oxidante, por lo que se deduce que la reacción se ve favorecida en presencia de oxígeno. La gráfica refleja un comportamiento similar para todos los sólidos basados en la saponita pilareada, alcanzando conversiones de propeno cercanas al $80 \%$ y de $\mathrm{NO}_{\mathrm{x}}$ del $40 \%$. Al igual que en los estudios anteriores, se observa que la producción de nitrógeno transcurre de forma paralela a la reducción de óxidos de nitrógeno, por lo que no se forman productos de reducción intermedios. De nuevo, el catalizador impregnado sobre alúmina presenta un comportamiento diferente, alcanzando mayores 
conversiones tanto para la oxidación de propeno como para la reducción del NO. Como se ha comentado anteriormente esto puede ser atribuido a las propiedades superficiales que presenta dicho catalizador. Estos resultados ponen de manifiesto el efectivo comportamiento redox del cromo que se ve favorecido en presencia de oxígeno.

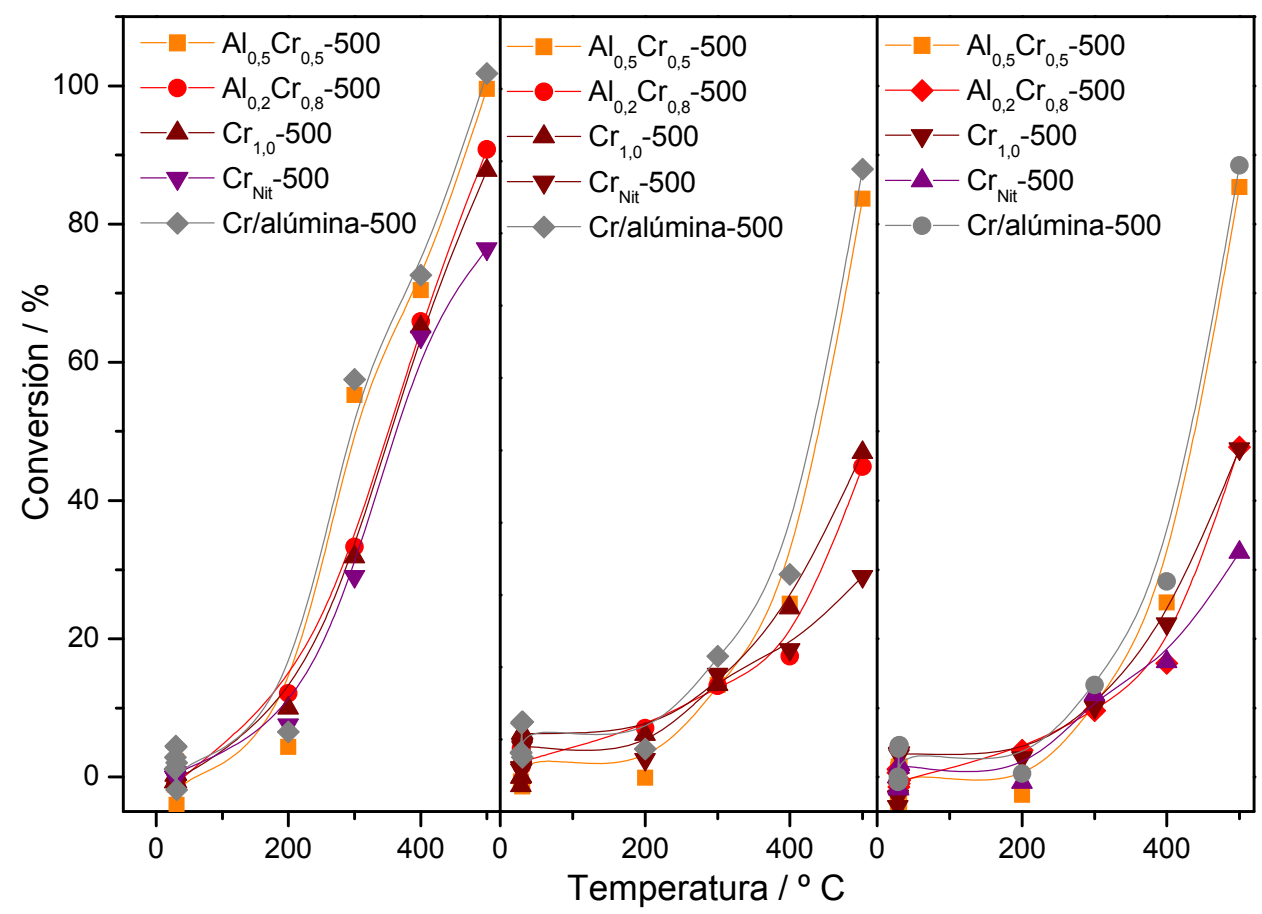

Figura 6. Conversiones de hidrocarburo y $\mathrm{NO}_{x}$ y rendimiento a nitrógeno obtenidas en la reacción $\mathrm{C}_{3} \mathrm{H}_{6}+4,5 \mathrm{NO}+2,25 \mathrm{O}_{2}$ para los catalizadores impregnados y pilareados que se indican.

Con objeto de evaluar el efecto del oxígeno ante la reacción conjunta de $\mathrm{NO}+\mathrm{O}_{2}$, se llevaron a cabo ensayos catalíticos introduciendo cantidades crecientes de oxígeno en condiciones isotermas. Los resultados obtenidos se muestran en la figura 7 para uno de los sólidos pilareados escogidos como modelo, ya que todos los sólidos estudiados presentaron el mismo comportamiento, incluso el preparado sobre alúmina como soporte, representándose los resultados registrados a 400 y $500{ }^{\circ} \mathrm{C}$.

Al evaluar el efecto del oxígeno se observa como a temperaturas intermedias, en las que la conversión del hidrocarburo no ha llegado a su máximo valor $\left(400{ }^{\circ} \mathrm{C}\right)$, la incorporación de oxígeno favorece la oxidación del hidrocarburo sin producir un detrimento en la reducción de los óxidos de nitrógeno, incluso para elevadas concentraciones de oxígeno. Esto pone de manifiesto la existencia de un efecto sinérgico entre el oxígeno y el NO a estas temperaturas. Sin embargo 
realizando el mismo estudio a $500{ }^{\circ} \mathrm{C}$, temperatura a la que la conversión de hidrocarburo casi ha alcanzado el $100 \%$, se observa como el exceso de oxígeno introducido compite con los $\mathrm{NO}_{\mathrm{x}}$ como agente oxidante del propeno. A esta temperatura el propeno reacciona preferentemente con el oxígeno, produciéndose un importante descenso en la conversión de $\mathrm{NO}_{\mathrm{x}}$.

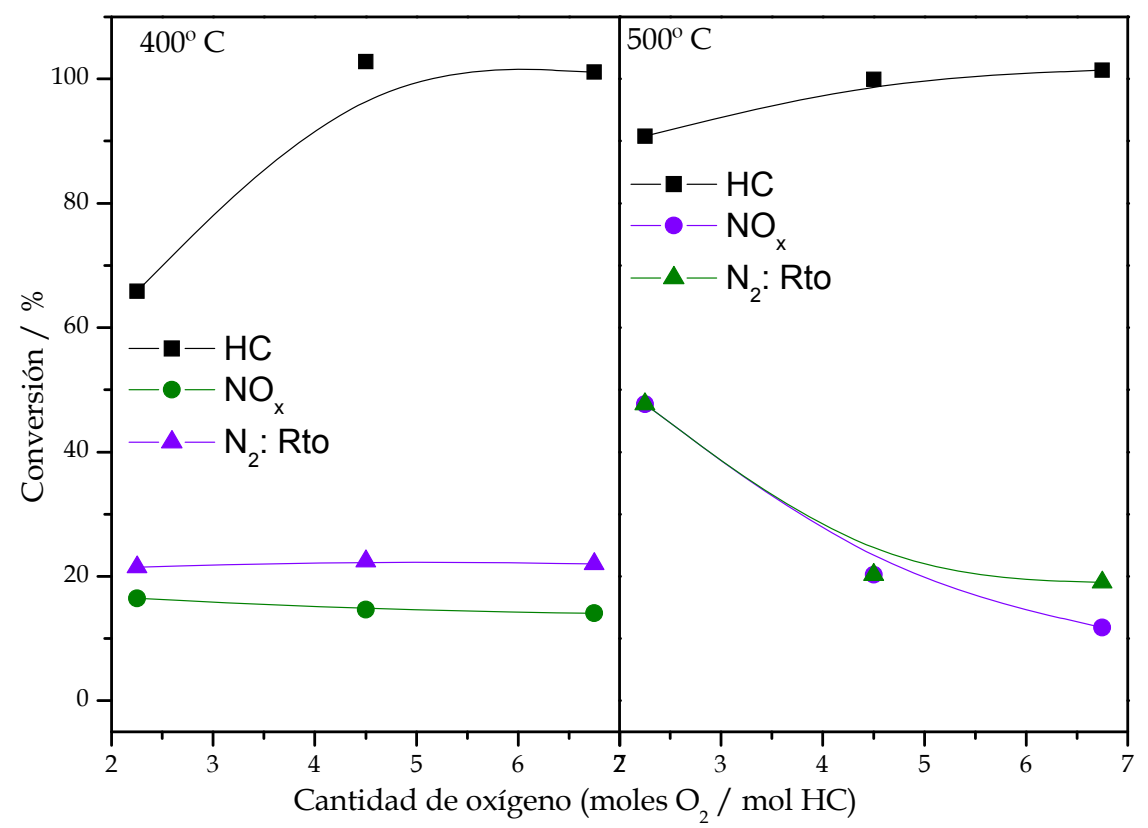

Figura 7. Conversiones de hidrocarburo y $\mathrm{NO}_{x}$ y rendimiento a nitrógeno obtenidos para el catalizador $\mathrm{Al}_{0,2} \mathrm{Cr}_{0,8}$. Izquierda: reacción a $400{ }^{\circ} \mathrm{C}$ y derecha: reacción a $500{ }^{\circ} \mathrm{C}$.

Estos resultados ponen de manifiesto la eficacia de los sólidos cromo-arcilla como catalizadores para la oxidación del hidrocarburo, existiendo unas condiciones óptimas que afectan al contenido en cromo y a la naturaleza del agente oxidante más que a la forma de incorporar la fase activa en el catalizador.

\section{V.6 Conclusiones}

La intercalación de una saponita con $\mathrm{Al}, \mathrm{Cr}$, o mezclas de ambos elementos se realiza de forma satisfactoria en todas las muestras preparadas. Los difractogramas de las muestras calcinadas indican que los policationes de cromo son menos estables a altas temperaturas. Introduciendo la relación adecuada $\mathrm{Al} / \mathrm{Cr}$ podemos obtener sólidos que combinan adecuadamente la estabilidad de los pilares de aluminio con las propiedades catalíticas aportadas por el cromo. En el caso de los sólidos impregnados, la calcinación produce, en algunos casos, la deslaminación de las muestras dependiendo de la sal utilizada como precursor para la impregnación. 
La cantidad de cromo final de los catalizadores puede controlarse preparando las disoluciones intercalantes o de impregnación adecuadas al catalizador deseado.

Los sólidos calcinados presentan elevados espaciados basales y superficies específicas, características que los hacen adecuados para su uso como catalizadores de oxidación. La actividad de los sólidos preparados frente a la oxidación de propeno y a la reducción de $\mathrm{NO}_{\mathrm{x}}$ es muy similar independientemente del método utilizado para la incorporación de cromo. En función de las condiciones oxidantes utilizadas en la alimentación se obtienen resultados diferentes tanto en la combustión de hidrocarburos como en la reducción de $\mathrm{NO}_{\mathrm{x}}$.

\section{IV.7. Bibliografía}

[1] A. Gil, L.M. Gandía y M.A. Vicente. Catal. Rev. Sci. Eng. 42, 145-212 (2000).

[2] L. Storaro, R. Ganzerla, M. Lenarda, R. Zanoni, A. Jiménez-López, P. OliveraPastor, E. Rodríguez-Castellón. J. Mol. Catal. A: Chemical, 115, 329-338 (1997).

[3] P. Olivera-Pastor, J. Maza-Rodríguez, P. Maireles-Torres, E. RodríguezCastellón, A. Jiménez-López. J. Mater. Chem. 4, 179-184, (1995).

[4] B. Grzybowska, J. Sloczynski, L. Kerommes, K. Wcislo, T. Bobinska. Appl. Catal. A: General, 209, 279-289, (2001).

[5] M.C.M. Alvim Ferraz, S. Möser, M. Tonhäeuser. Fuel, 78, 1567-1573, (1999).

[6] M. Kang, C. Lee. Appl. Catal. A: General, 266, 163-172, (2004).

[7] M.A. Vicente, A. Meyer, E. González, M.A. Bañares-Muñoz, L.M. Gandía, A. Gil. Catal. Lett., 78, 99-103, (2002).

[8] J.R. Sohn, S. Gon Ryu. Catal, Lett., 78, 105-110, (2001).

[9] C. Volzone. Clays Clay Miner., 43, 377-382 (1995).

[10] G.W. Brindley y S. Yamakana. Am. Miner., 64, 830-835 (1979).

[11] N. Lahav, U. Shani y J. Shabtai. Clays Clay Miner., 26, 107-115, (1978).

[12] A. Iglesias-Juez. Tesis Doctoral, Universidad Autónoma de Madrid (2004).

[13] C. Belver, M.A. Vicente, A. Martínez-Arias, M. Fernández-García. Appl Catal. B: Environ., 50, 227-234 (2004).

[14] F. Radtke, R.A. Köppel y A. Baiker. Catal. Today, 26, 159-167 (1995). 



\section{Capítulo V}

$\mathrm{Ni}$-pillared clays as catalysts for the selective catalytic reduction of nitrogen oxides 



\section{Abstract}

Different and alternative methods for the preparation of $\mathrm{Ni}$-pillared clays based on impregnation and encapsulation procedures are reported. Several nickel precursors and metal loads are evaluated in order to assess their influence in the structural, textural and catalytic properties of the resulting solids. The behaviour of the optimum solids as catalysts has been proved in the selective catalytic reduction of nitrogen oxides with propene. The redox properties of nickel species induce the reduction of the nitrogen oxides at low temperatures whereas the hydrocarbon oxidation is enhanced at higher temperatures.

Keywords: Pillared clays, Ni catalysts, DeNOx reaction

\section{V.1. Introduction}

Clays are versatile materials employed in diverse applications, comprising from ceramics to catalysts. Their utilization is mainly based on their properties, surface, acidity and/or composition [1,2]. In this sense, many tailoring procedures have been performed in order to improve different clays, intercalation-pillaring being one of the most studied [3,4]. Pillared layered clays appear as new microporous materials whose properties can be modulated by selecting the adequate synthetic parameters, such as nature of the pillaring agent, type of clay or thermal treatment; thus proffering an important way to design tailored catalysts [5].

The synthesis of pillared clays (PILCS) is based in the ionic exchange of the original interlayer cations by polyoxocations previously prepared. The large intercalated polyoxocations are thermally transformed into stable metallic oxidic clusters grafted to the clay layers, forming interlamellar galleries accessible to small and medium size molecules [6]. The catalytic applications of PILCs depend on the nature of both the parent clay and the intra-layer pillars. The pillars formation permits to improve the textural properties by the creation of intra-galleries and also to create promising active centres (acidic or metallic). The presence of transition metals in the pillars has proved to be useful in oxidation-reduction reactions, such as oil cracking, fine chemical synthesis $[7,8]$ or selective catalytic reduction, using ammonia or hydrocarbon as reducing agents $[9,10]$. 
The selective catalytic reduction of nitrogen oxides by hydrocarbons (HC-SCR) has attracted much attention since it appears as a potential way to remove these pollutants from motor exhausts, avoiding the inconveniences derived from the use of ammonia. So far, the most common De- $\mathrm{NO}_{x}$ catalysts employed are based on transition and noble metals supported over zeolites or alumina [11-13]. The use of metal pillared clays in this reaction has not been enlarged, finding some reports based on Fe-, Co-, Cr-, and Cu-PILCs [14-16], and more recently noble metal PILCs [17]. Thus, we have focused our attention both on pillared clays, as optimum microporous materials for this reaction, and on $\mathrm{Ni}$-catalysts, described in the literature as effective for $\mathrm{NO}_{x}$ reduction in presence of $\mathrm{C}_{2}$ and $\mathrm{C}_{3}$ hydrocarbons $[18,19]$.

In this study, Ni-saponite catalysts were prepared by two different ways; impregnation of Al-pillared saponite with several nickel precursors, and encapsulation of nickel complexes in the parent and in the intercalated clay. The resulting materials were characterized and evaluated as catalysts for selective catalytic reduction of nitrogen oxides with propene.

\section{V.2. Experimental}

\section{V.2.1. The Starting Clay}

The clay used in this work was the saponite from Cabañas (Toledo, Spain), supplied by TOLSA (Madrid), previously purified by dispersion-decantation of the $<2 \mu \mathrm{m}$ fraction [20]. Its cation exchange capacity was 0.99 mequiv/g, its basal spacing $12.2 \AA$ and the BET specific surface area $191 \mathrm{~m}^{2} / \mathrm{g}$.

\section{V.2.2. Synthesis of $\mathrm{Ni}-$ Clay Catalysts}

The synthesis of the Al-pillared saponite was carried out by intercalation of $\mathrm{Al}_{13}$ polycations by a standard procedure [21]. $\mathrm{Al}_{13}{ }^{7+}$ polycations were prepared by controlled hydrolysis of an $\mathrm{Al}^{3+}$ solution (obtained from $\mathrm{AlCl}_{3} \cdot 6 \mathrm{H}_{2} \mathrm{O}$ ) with $1 \mathrm{M} \mathrm{NaOH}$, using a ratio $\mathrm{OH}^{-} / \mathrm{Al}^{3+}=2.2$. The hydrolysis reaction was maintained under vigorous stirring during $24 \mathrm{~h}$. Then, the solution was added to a previously prepared clay suspension employing an $\mathrm{Al} / \mathrm{clay}$ ratio of $5 \mathrm{mmol} / \mathrm{g}$, aging the new suspension 
under stirring for $24 \mathrm{~h}$. The resulting solid was separated by centrifugation, washed

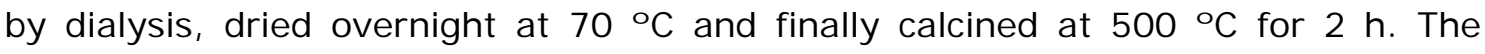
sample thus synthesized, pillared saponite, was designated as AI-PILC (in some cases, the citation to Al-intercalated saponite would refer to the sample before calcination, only dried).

The Al-pillared saponite above synthesized was used as support for the preparation of the first series of nickel-catalysts. Nickel was incorporated by a standard incipient wetness impregnation method [22]. Thus, $\mathrm{Ni}(\mathrm{II})$ nitrate, $\mathrm{Ni}(\mathrm{II})$ chloride and $\mathrm{Ni}(\mathrm{II})$ acetylacetonate salts (Sigma-Aldrich) were chosen as $\mathrm{Ni}(\mathrm{II})$ precursors. Impregnation with $\mathrm{Ni}$ chloride was performed in order to obtain catalysts with $\mathrm{NiO}$ loading from 1 to 3 wt. \%, nevertheless preliminary catalytic results made necessary to increase the nickel amounts to obtain catalysts with $\mathrm{NiO}$ loading from 1.5 to $6 \mathrm{wt}$. \%. The precursors were dissolved in the minimum solvent amount (acetone for acetylacetonate salt and water for the rest) and the solution was added to the AI-PILC support. The resulting solids were dried and calcined following the same protocol above reported. The solids were designated as followed: $\mathrm{Ni}($ nit), $\mathrm{Ni}(\mathrm{acac})$ and $\mathrm{NiCl}$, indicating the metal precursor, $\mathrm{Ni}(\mathrm{II})$ nitrate, acetylacetonate and chloride, respectively; followed by a number that indicates the formal NiO load of the catalyst.

The second series of nickel catalysts was prepared by encapsulation of a nickel complex both in the natural and in the Al-intercalated saponite. This procedure has been recently developed in order to combine the chemical selectivity of the metal complex with the stability of heterogeneous supported catalysts [23]. Thus, $\mathrm{Ni}(\mathrm{II})$ phtalocianine (Sigma-Aldrich) was chosen as Ni-complex using pyridine (SigmaAldrich) as solvent. The encapsulation procedure was performed by putting in touch the clay with a large amount of the complex for $72 \mathrm{~h}$, replacing the evaporated solvent during the process. The solids obtained were separated by filtration, dried and calcined in the same way that the other nickel-samples. The solids were named Ns or Is, indicating the clay support (natural or intercalated saponite, respectively), followed by the abbreviation of the metal complex, Ph. Almost all data given correspond to the samples calcined at $500 \stackrel{\circ}{ } \mathrm{C}$, which are the final catalysts, when talking on dried samples, before calcination, the suffix dried is added to the name. 


\section{V.2.3. Characterization of the Solids}

Elemental analyses were carried out by Activation Laboratories Ltd., Ancaster, Ontario, Canada, using Inductively Coupled Plasma Optical Emission Spectroscopy (ICP OES).

Powder X-ray diffraction (PXRD) patterns were registered between 2 and 70 (20) with a scanning velocity of $2 \cong / m i n$ by using a Siemens D-500 diffractometer, operating at $40 \mathrm{kV}$ and $30 \mathrm{~mA}$, and employing $\mathrm{Cu} \mathrm{K \alpha}$ filtered radiation. The equipment was connected to a DACO-MP microprocessor and used Diffract-AT software.

Textural analyses were determined by nitrogen adsorption-desorption isotherms at $-196{ }^{\circ} \mathrm{C}$ using a Micromeritics apparatus (ASAP 2010). The samples were degassed for $6 \mathrm{~h}$ at room temperature and then for $2 \mathrm{~h}$ at $110{ }^{\circ} \mathrm{C}$. Specific surface area was obtained from the BET method, microporous surface area by means of the $\mathrm{t}$ method, and total pore volume from the adsorption at $\mathrm{p} / \mathrm{p}^{\circ} 0.99$.

DR-UV-Vis spectra were recorded in reflectance mode by using a Shimadzu spectrometer UV-2100, with an optical length of $0.4 \mathrm{~cm}$.

\section{V.2.4. HC-SCR Catalytic Activity}

The catalytic activity of each $\mathrm{Ni}$-catalyst was measured in a fixed bed flow reactor using a catalytic bed composed by the catalyst sieved mixed with SiC (ratio $1 / 2$ catalyst vs. $\mathrm{SiC}$ ). The catalyst amount and the total flow were adjusted in order to work with a gas hourly space velocity of $50000 \mathrm{~h}^{-1}$. The HC-SCR reaction was evaluated under stoichiometric conditions, adjusting the reactant gas (v/v) at $0.1 \% \mathrm{C}_{3} \mathrm{H}_{6}$ (L'Air Liquide) and $0.9 \% \mathrm{NO}_{x}$ ( $\mathrm{NO} 98 \% ; \mathrm{NO}_{2} 1 \% ; \mathrm{N}_{2} \mathrm{O} 0.5 \%$, Carburos Metálicos, Spain) in nitrogen (L'Air Liquide).

The reaction was carried out under stationary conditions from 200 to $500 \stackrel{\circ}{ }$, using a heating rate of $5 \stackrel{\circ}{\circ} \mathrm{min}^{-1}$ and a time stabilization of $25 \mathrm{~min}$, recording data at intervals of $50 \stackrel{\circ}{ } \mathrm{C}$. The outflow gas was analyzed by on-line infrared spectroscopy using a Perkin-Elmer 1725X FTIR spectrometer using a multiple reflection transmission cell (Infrared Analysis Inc.). Catalytic activities for $\mathrm{NO}_{x}$ were evaluated in terms of percentage conversions of $\mathrm{NO}$ and $\mathrm{NO}_{2}$ to $\mathrm{N}_{2}$, because $\mathrm{N}_{2} \mathrm{O}$ formation was negligible in all conditions studied. 


\section{V.3. Results and Discussion}

\section{V.3.1. Preparation and Characterization of Ni-clays}

The intercalation-pillaring process with Al was successful, increasing the basal spacing from $13.8 \AA$ in the natural clay, to $18.2 \AA$ in the intercalated solid and $17.8 \AA$ in the pillared one (Figure 1 ).

The impregnated samples maintain the layered structure of the Al-PILC support although the c-axis ordering decreases, indicating some delamination after impregnation (Figure 1). The behaviour of the three nickel precursors is quite similar, suggesting a high dispersion of the Ni species on the support surface; only the impregnation with high amounts of acetylacetonate causes the formation of detectable crystallites of $\mathrm{NiO}$ bunsenite phase (JCPDS: 4-0835), see $\mathrm{Ni}(\mathrm{acac}) 6$ diffractogram. This fact could be due to a worse dispersion of nickel during the impregnation with this precursor, because of the high volatility of the acetone used as solvent.

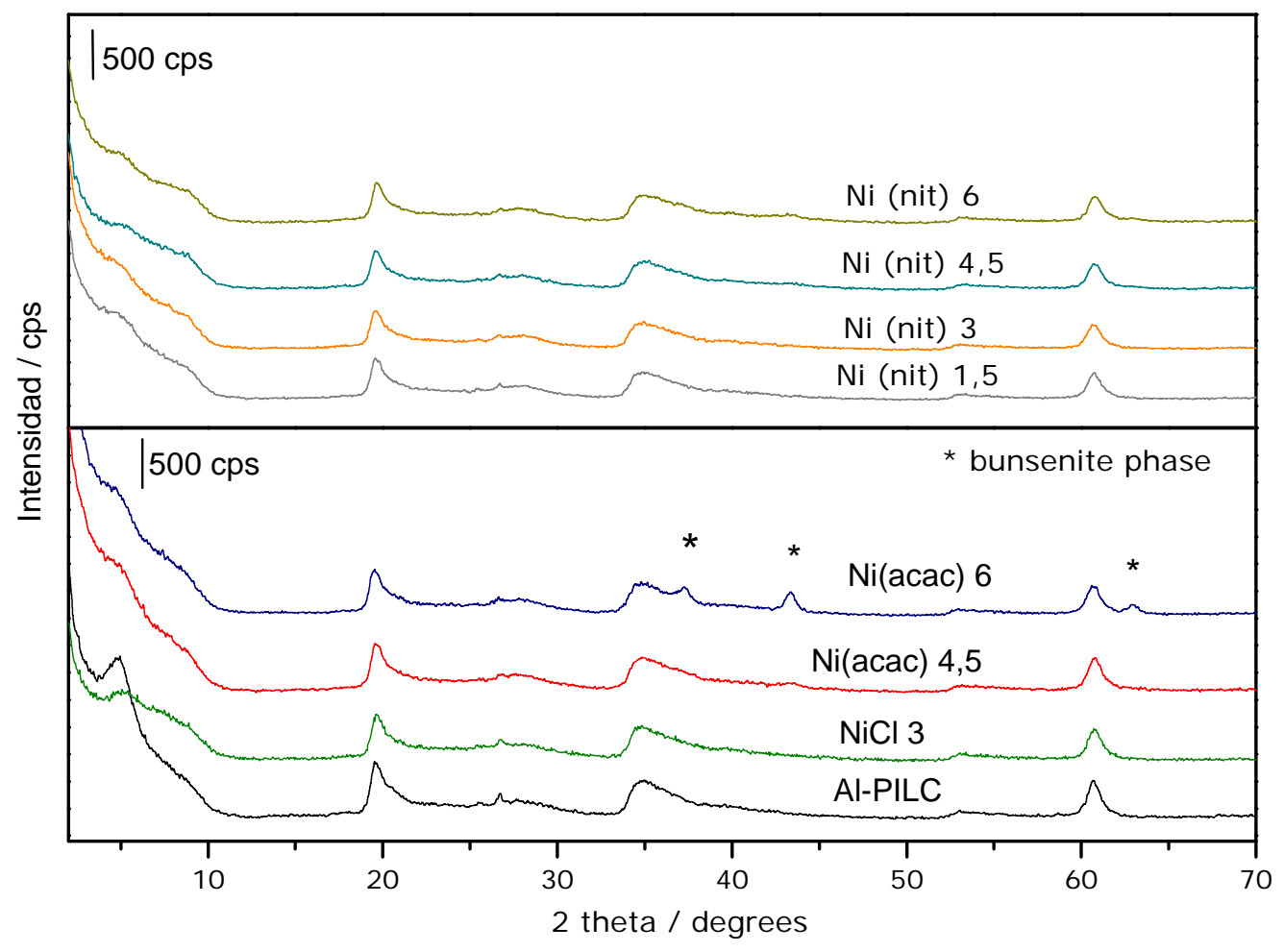

Figure 1. XRD patterns of the impregnated solids indicated; the diffractogram of the support, Al-PILC solid, is shown as reference. 


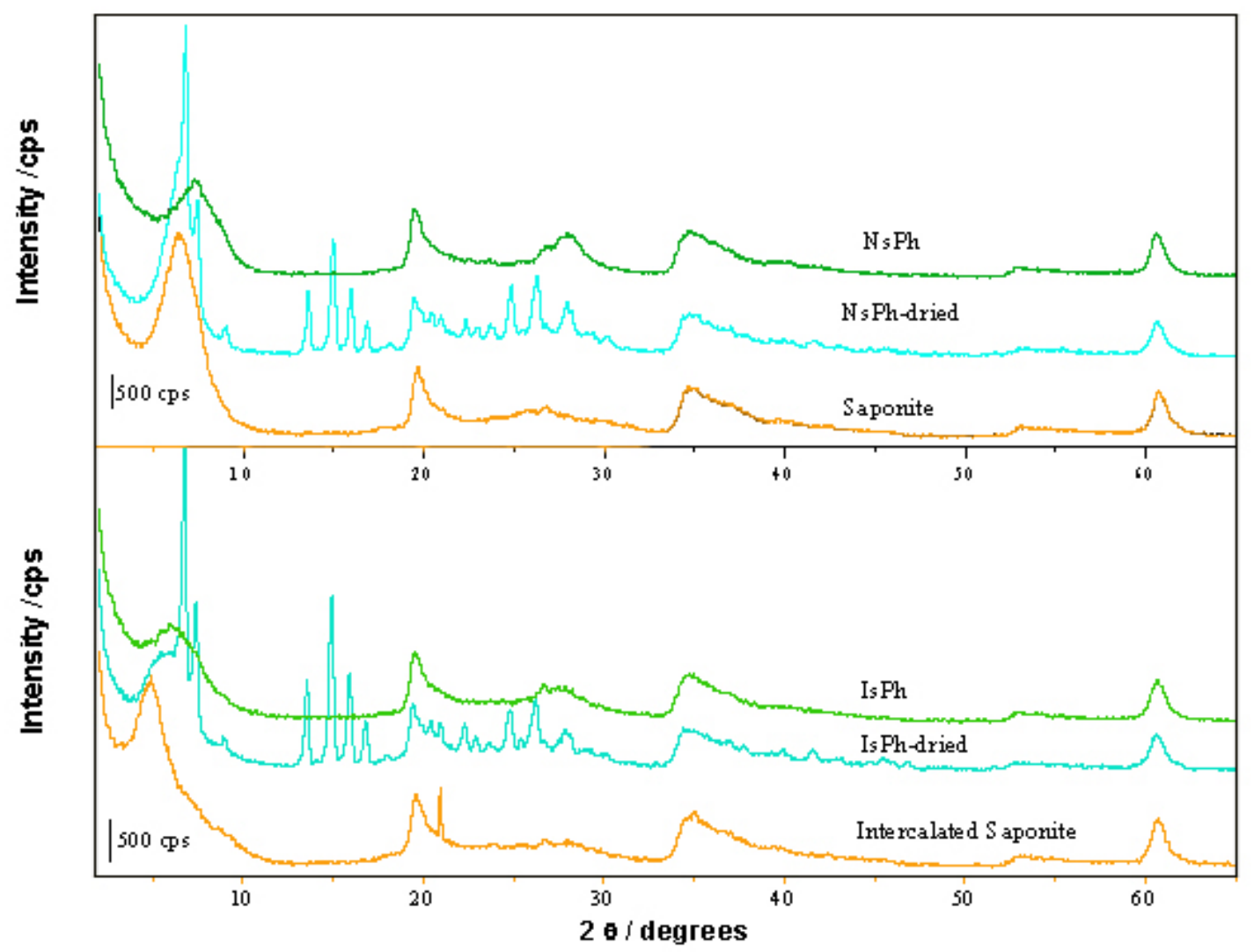

Figure 2. XRD patterns of the encapsulated solids; the diffractograms of parent and intercalated saponite are shown as reference.

The encapsulation process over the natural saponite leads to solids with basal spacing of 13.1 , with a shoulder at 14.6 , and $12.9 \AA$, respectively, when using both supports, natural and Al-intercalated saponite, together with all the characteristic peaks from the supports and from the $\mathrm{Ni}$-complexes (Figure 2). For intercalated saponite, it is evident that the interaction with the organic molecule provokes the collapse of $\mathrm{Al}_{13}{ }^{7+}$ units; though it may be considered that the $\mathrm{Al}$-intercalated clay is used for this treatment, thus $\mathrm{Al}_{13}$ species have not been still transformed into pillars and are not linked to the clay layers but as polycationic species. Looking at the chemical composition of the solids and the thermal analyses (not shown), it is deduced that the nickel complex is situated in the interlayer space of natural saponite by substitution of water molecules, being intercalated as neutral molecules, cationic exchange is not observed. As water is removed, the basal spacing of the intercalated solids may be compared to that of the TOT structure of 
saponite $(9.6 \AA)$, and thus the separation of the layers produced by the complex being of 3.3-3.5 $\AA$. The form of the molecule allows packing a large number of them in the interlayer spacing, giving rise to high final nickel content. When these solids are calcined at $500 \stackrel{\circ}{C}$, the characteristic diffractogram of the clay is observed, with intense peaks at 14.6 and $12.0 \AA$, not showing any effect due to the formation of NiO crystallites.

\begin{tabular}{|c|c|c|c|c|c|c|c|c|c|c|}
\hline Sample & $\mathrm{SiO}_{2}$ & $\mathrm{Al}_{2} \mathrm{O}_{3}$ & $\mathrm{Fe}_{2} \mathrm{O}_{3}$ & $\mathrm{MnO}$ & $\mathrm{MgO}$ & $\mathrm{CaO}$ & $\mathrm{Na}_{2} \mathrm{O}$ & $\mathrm{K}_{2} \mathrm{O}$ & $\mathrm{TiO}_{2}$ & $\mathrm{NiO}$ \\
\hline Saponite & 58.8 & 6.3 & 1.9 & 0.11 & 31.4 & 0.49 & 0.08 & 0.55 & 0.25 & -- \\
\hline Al-PI LC & 54.6 & 14.4 & 1.8 & 0.10 & 28.1 & 0.02 & 0.09 & 0.44 & 0.22 & -- \\
\hline $\mathrm{NiCl} 1$ & 54.5 & 15.1 & 1.9 & 0.11 & 26.4 & 0.03 & 0.08 & 0.58 & 0.24 & 0.9 \\
\hline $\mathrm{NiCl} 1.5$ & 53.8 & 15.0 & 2.3 & 0.11 & 26.2 & 0.03 & 0.08 & 0.61 & 0.24 & 1.5 \\
\hline $\mathrm{NiCl} 2$ & 53.5 & 14.8 & 1.7 & 0.11 & 26.0 & 0.03 & 0.07 & 0.55 & 0.23 & 2.8 \\
\hline $\mathrm{NiCl} 3$ & 52.8 & 14.9 & 2.2 & 0.10 & 25.9 & 0.03 & 0.08 & 0.59 & 0.23 & 3.3 \\
\hline $\mathrm{Ni}($ acac $) 1.5$ & 54.4 & 15.0 & 2.2 & 0.11 & 26.4 & 0.03 & 0.08 & 0.59 & 0.24 & 1.08 \\
\hline $\mathrm{Ni}($ acac $) 3$ & 53.1 & 14.7 & 2.0 & 0.10 & 25.9 & 0.03 & 0.08 & 0.60 & 0.23 & 3.2 \\
\hline $\mathrm{Ni}($ acac $) 4.5$ & 53.1 & 14.7 & 1.7 & 0.10 & 25.8 & 0.03 & 0.08 & 0.55 & 0.23 & 3.5 \\
\hline $\mathrm{Ni}($ acac $) 6$ & 51. 9 & 14.4 & 1.7 & 0.10 & 25.3 & 0.03 & 0.08 & 0.56 & 0.23 & 5.6 \\
\hline $\mathrm{Ni}($ nit) 1.5 & 53.3 & 14.7 & 3.3 & 0.10 & 25.9 & 0.03 & 0.08 & 0.54 & 0.23 & 1.7 \\
\hline $\mathrm{Ni}($ nit) 3 & 53.6 & 14.8 & 1.6 & 0.10 & 26.1 & 0.02 & 0.08 & 0.60 & 0.23 & 2.7 \\
\hline $\mathrm{Ni}($ nit) 4.5 & 52.2 & 14.5 & 2.2 & 0.10 & 25.5 & 0.02 & 0.07 & 0.58 & 0.23 & 4.4 \\
\hline $\mathrm{Ni}($ nit) 6 & 51.9 & 14.4 & 1.8 & 0.10 & 25.4 & 0.03 & 0.08 & 0.62 & 0.23 & 5.3 \\
\hline $\mathrm{NsPh}$ & 53.2 & 6.4 & 2.0 & 0.12 & 29.6 & 0.51 & 0.11 & 0.63 & 0.25 & 7.0 \\
\hline IsPh & 55.0 & 15.4 & 2.1 & 0.09 & 26.2 & 0.14 & 0.23 & 0.05 & 0.28 & 0.003 \\
\hline
\end{tabular}

Table 1. Chemical composition of the natural clay, the pillared clay and the nickel samples (wt. \%, water free solids).

The nitrogen adsorption-desorption isotherms of all solids are of type II (IUPAC classification, not shown) with a hysteresis loop type $\mathrm{H} 3$ and a high adsorption at low relative pressures. This behaviour is characteristic of layered materials with slitlike pores and microporosity [24,25]. The textural parameters of the solids obtained from these isotherms are listed in Table 2. 


\begin{tabular}{cccc}
\hline Sample & $\mathrm{S}_{\text {BET }} / \mathrm{m}^{2} \mathrm{~g}^{-1}$ & $\mathrm{~S}_{\mathrm{MP}} / \mathrm{m}^{2} \mathrm{~g}^{-1}$ & $\mathrm{~V}_{\mathrm{T}} / \mathrm{cm}^{3} \mathrm{~g}^{-1}$ \\
\hline Saponite & 191 & 143 & 0.157 \\
$\mathrm{Al}$-PILC & 230 & 180 & 0.176 \\
\hline $\mathrm{NiCl} 1$ & 159 & 115 & 0.137 \\
$\mathrm{NiCl} 1.5$ & 145 & 104 & 0.128 \\
$\mathrm{NiCl} 2$ & 137 & 100 & 0.121 \\
$\mathrm{NiCl} 3$ & 102 & 71 & 0.102 \\
\hline $\mathrm{Ni}$ (acac) 1.5 & 182 & 129 & 0.167 \\
$\mathrm{Ni}$ (acac)3 & 186 & 131 & 0.167 \\
$\mathrm{Ni}$ (acac)4.5 & 183 & 131 & 0.165 \\
$\mathrm{Ni}$ (acac)6 & 183 & 131 & 0.166 \\
\hline $\mathrm{Ni}($ nit)1.5 & 169 & 123 & 0.147 \\
$\mathrm{Ni}$ (nit)3 & 154 & 113 & 0.134 \\
$\mathrm{Ni}$ (nit)4.5 & 151 & 118 & 0.126 \\
$\mathrm{Ni}$ (nit)6 & 146 & 120 & 0.102 \\
\hline $\mathrm{NsPh}$ & 137 & 85 & 0.136 \\
$\mathrm{IsPh}$ & 138 & 85 & 0.134 \\
\hline
\end{tabular}

Table 2. BET specific surface area $\left(\mathrm{S}_{\mathrm{BET}}\right)$, microporous area $\left(\mathrm{S}_{\mathrm{MP}}\right)$ and total volume $\left(\mathrm{V}_{\mathrm{T}}\right)$ of the solids indicated.

Natural saponite shows a high specific surface area value, characteristic of saponites in Madrid Basin, due to their small particle size, derived from the sedimentary origin of these deposits $[26,27]$. The specific surface area and the microporosity increase after pillaring process but decrease during the impregnation procedure, due to the calcination necessary for the elimination of the precursors. All nickel catalysts posses optimum surface areas and microporosity for catalytic applications. The acetylacetonate impregnation appears as the best way to obtain materials with high surface areas, around $180 \mathrm{~m}^{2} \mathrm{~g}^{-1}$ with a $70 \%$ of microporous surface area. Both encapsulated solids, also studied after calcination at $500{ }^{\circ} \mathrm{C}$, have very similar textural parameters, with the surface area close to $137 \mathrm{~m}^{2} / \mathrm{g}$.

The nickel species present in the samples were studied by DR-UV-Vis spectroscopy, viewing the electronic spectra of the impregnated samples in Figure 3. 


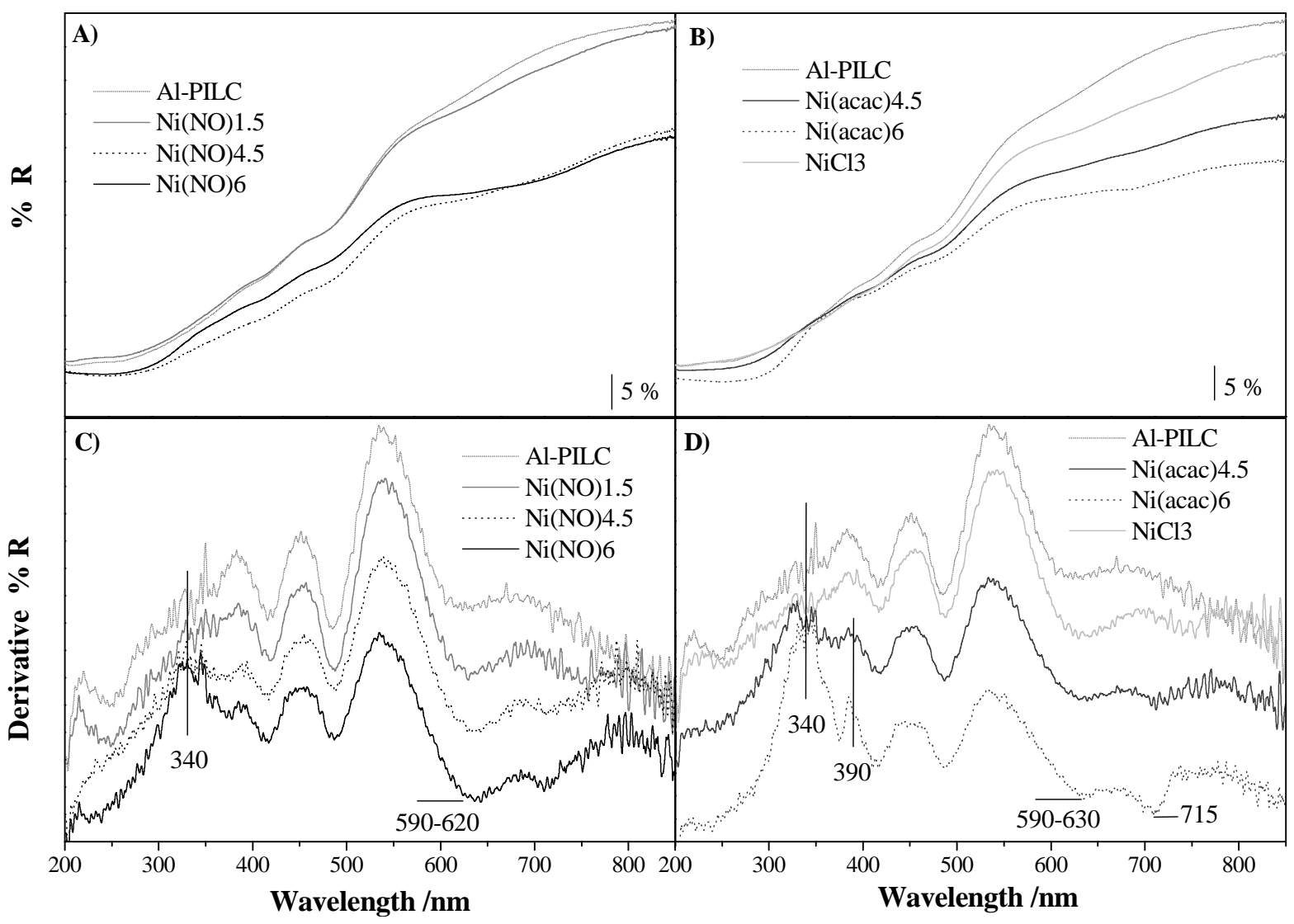

Figure 3. DR-UV-Vis spectra of impregnated solids (A, B) and their respective derivative curves (C, D).

The nickel-supported solids exhibit similar electronic spectra than the support (figure $3 A, B$ ); only in the derivative curves it is possible to observe some differences (figure $3 C, D$ ). According to the literature $[28,29]$, it is possible to distinguish the $d-d$ transitions of $\mathrm{Ni}^{2+}$ ions in different environments. Around 350 $\mathrm{nm}$ appears an absorption band attributed to the ${ }^{3} \mathrm{~A}_{2 g}-{ }^{1} \mathrm{~T}_{1 \mathrm{~g}}$ (D) transition of octahedral $\mathrm{Ni}(\mathrm{II})$ in $\mathrm{NiO}$. At higher wavelengths, and overlapping with an absorption band of the Al-PILC, a shoulder becomes visible at around 590-650 nm, probably associated to the ${ }^{3} \mathrm{~T}_{1}-{ }^{1} \mathrm{~T}_{2}$ and ${ }^{3} \mathrm{~T}_{1}-{ }^{3} \mathrm{~T}_{1}(\mathrm{P})$ transitions of tetrahedral $\mathrm{Ni}(\mathrm{II})$ ions in the alumina lattice, attributed to the presence of $\mathrm{NiAl}_{2} \mathrm{O}_{4}$ spinel [30]. Comparing the impregnated solids, the $\mathrm{Ni}$ (nit) series only shows evidences of these absorption bands in the samples with high nickel content ( $3 \%$ ), as also occurs for the $\mathrm{NiCl}$ series, while in the $\mathrm{Ni}$ (acac) series, this absorption becomes more evident. If figure $3 \mathrm{D}$ is analysed in detail, it is possible to observe that the band of the support at 
$400 \mathrm{~nm}$ almost disappears in the $\mathrm{Ni}(\mathrm{acac}) 4.5$ sample, appearing other one at $390 \mathrm{~nm}$ in the $\mathrm{Ni}(\mathrm{acac}) 6$ sample. According to the literature this band is attributed to the presence of $\mathrm{NiAl}_{2} \mathrm{O}_{4}$ spinel, assigned to the ${ }^{3} \mathrm{~A}_{2 g}-{ }^{1} \mathrm{~T}_{1 \mathrm{~g}}(\mathrm{P})$ transition of octahedral $\mathrm{Ni}(\mathrm{II})$ in the alumina lattice [30]. The $\mathrm{Ni}($ acac) 6 solid also presents other band at $715 \mathrm{~nm}$ probably associated to the ${ }^{3} \mathrm{~A}_{2 g}-{ }^{3} \mathrm{~T}_{1 \mathrm{~g}}(\mathrm{~F})$ transition of octahedral $\mathrm{Ni}(\mathrm{II})$ in NiO. These results suggest that the impregnation synthetic process yields to similar nickel species for all samples, being the $\mathrm{Ni}(\mathrm{II})$ acetylacetonate the optimum precursor for obtaining higher amounts of $\mathrm{NiO}$ and $\mathrm{NiAl}_{2} \mathrm{O}_{4}$ species; whose presence could be relevant for catalytic applications. The encapsulated samples could not be studied by this technique due to their intense black colour, which produces a continuous light absorption.

\section{V.3.2. Catalytic Properties: Selective Catalytic Reduction of Nitrogen Oxides}

The $\mathrm{NO}_{\mathrm{x}}$ reduction with propene yields to $\mathrm{N}_{2}, \mathrm{CO}_{2}$ and carbon deposits as products for all solids studied, without evidences of $\mathrm{N}_{2} \mathrm{O}, \mathrm{NH}_{3}$ or other by-products. The activities of the Ni-clay catalysts are listed in Table 3.

\begin{tabular}{ccc}
\cline { 2 - 3 } & $\mathrm{C}_{3} \mathrm{H}_{6}$ conversion / \% & NOx conversion / \% \\
\hline $\mathrm{Ni}$ (nit) 1.5 & $26(400)$ & $15(400)$ \\
$\mathrm{Ni}($ nit)3 & $20(450)$ & $12(400)$ \\
$\mathrm{Ni}$ (nit)4.5 & $23(400)$ & $13(400)$ \\
$\mathrm{Ni}($ nit)6 & $22(450)$ & $19(450)$ \\
\hline $\mathrm{Ni}$ (acac)4.5 & $31(500)$ & $22(250)$ \\
$\mathrm{Ni}(\mathrm{acac}) 6$ & $37(500)$ & $16(500)$ \\
\hline $\mathrm{NiCl} 3$ & $22(500)$ & $17(300)$ \\
\hline $\mathrm{NsPh}$ & $27(500)$ & $18(300)$ \\
IsPh & $30(500)$ & $17(450)$ \\
\hline
\end{tabular}

Table 3. Activity of $\mathrm{Ni}$-clays for the reduction of NOx with propene, expressed as the maximum conversion (in parenthesis, the temperature of maximum conversion for each reactant).

$\mathrm{NO}_{\mathrm{x}}$ and propene conversions are quite similar comparing the different samples studied, only the solids impregnated with acetylacetonate appear as more active. These conversion data, although not so high as other described for nickel catalysts [31,32], are analogous to those displayed for NiO and some Ni-ZSM-5 catalysts $[29,33]$. The presence of carbonaceous species, whose reversible formation is not 
easy, seems to be the main reason for this low activity [34]. In order to better compare the activity of the samples, the reaction rates are plotted in Figure 4, referred to the surface area of the solids, comparing the relevance of the Ni loading and of the precursor nature.

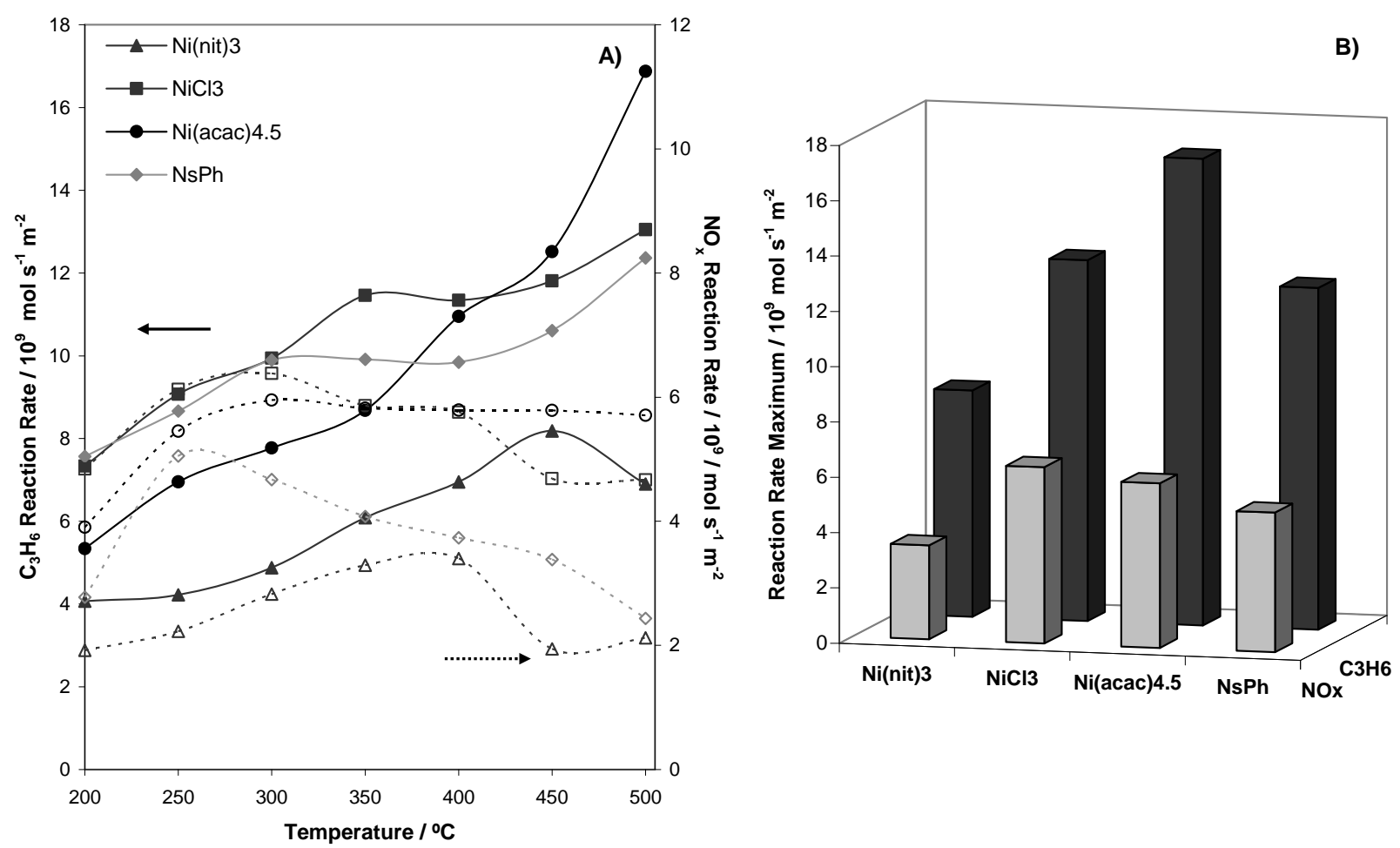

Figure 4. Reaction rates for hydrocarbon oxidation (full lines) and NOx reduction (dotted lines) comparing the $\mathrm{Ni}$-precursor and the synthesis procedure for similar nickel contents: a) following the reaction from 200 to $500 \stackrel{\circ}{ } \mathrm{C}$; b) maximum values.

The reaction rates for propene reaction are higher than for nitrogen oxides in all the solids studied. The main difference is related to the nature of the nickel precursor, $\mathrm{Ni}(\mathrm{acac})$ being the series the most active. In this sense, the literature has reported that the presence of $\mathrm{NiO}$ and $\mathrm{NiAl}_{2} \mathrm{O}_{4}$ species is related to the $\mathrm{SCR}$ activity [32,35], although the acidity properties also possess relevance [31]. The $\mathrm{NO}_{x}$ reduction is mainly related to the surface $\mathrm{NiAl}_{2} \mathrm{O}_{4}$-like phase whereas the $\mathrm{C}_{3} \mathrm{H}_{6}$ oxidation depends on the amount of highly dispersed NiO phase [29]; therefore, the $\mathrm{Ni}(\mathrm{acac})$ series with higher amounts of $\mathrm{NiO}$ and $\mathrm{NiAl}_{2} \mathrm{O}_{4}$ results the most active. On the other side, the synthesis method does not play an important role in the optimisation of this reaction. The encapsulation procedure does not lead to better 
activities than impregnation process, even when the first samples possess higher nickel amounts. The encapsulated sample only shows higher activities than the series prepared from the nitrate precursor, probably due to the nickel species formed after thermal treatment.

The comparison of the samples derived from a precursor but with different nickel contents, displayed in Figure 5, shows a slight increasing of the activity for hydrocarbon oxidation, with a maximum located at $4 \%$ wt., while the $\mathrm{NO}_{\mathrm{x}}$ reduction only differs at the higher nickel contents. Although the literature describes an increasing activity related to the nickel loading in zeolites (associated to the amount of disperse $\mathrm{Ni}^{2+}$ cations $\left.[33,34]\right)$, it is important to indicate that the $\mathrm{NO}_{x}$ reduction is typically limited by the availability of reducing agent [31]. Therefore, these results suggest that at low-medium nickel contents the concentration of the reducing agent at the active sites is insufficient.
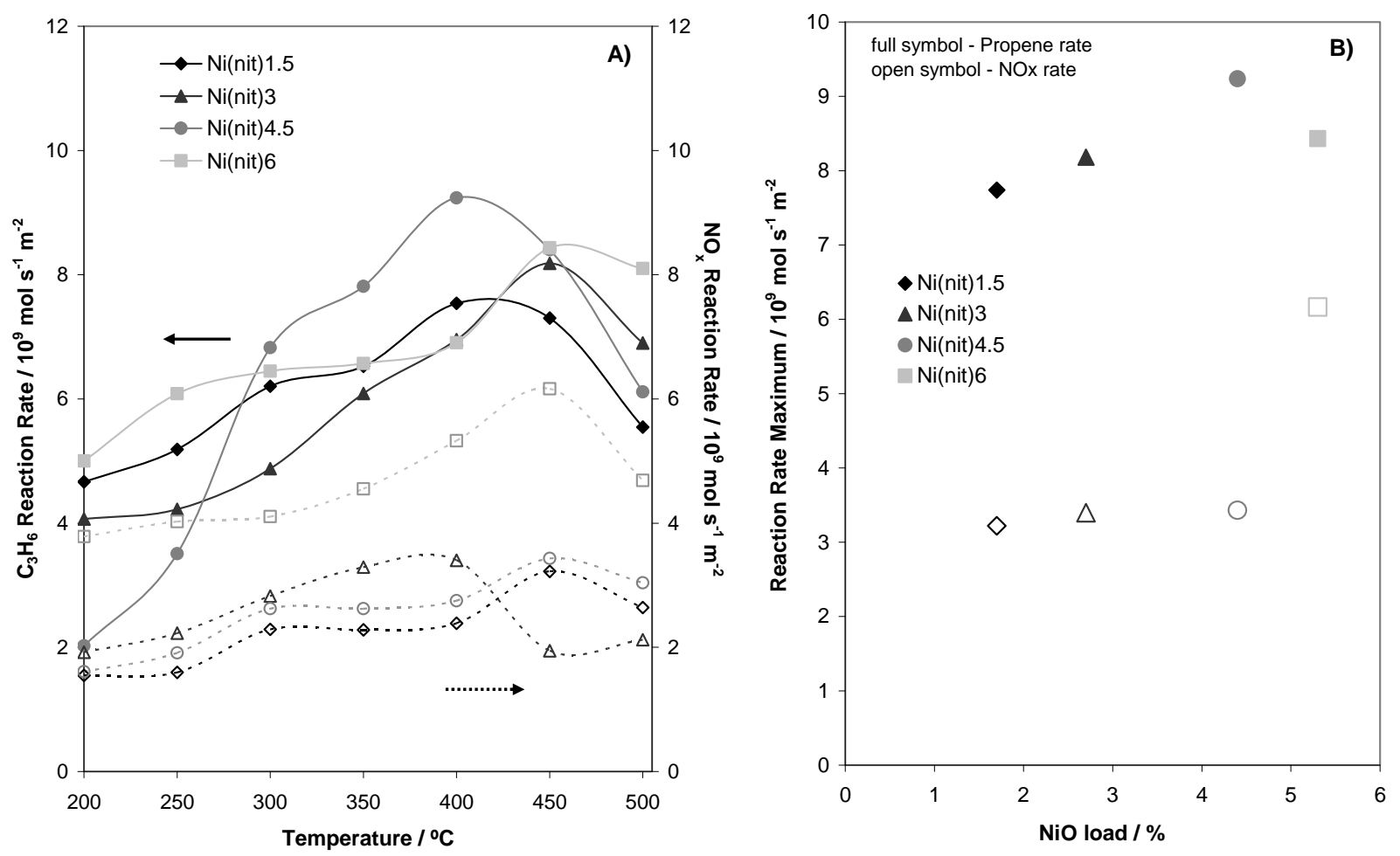

Figure 5. Reaction rates for hydrocarbon oxidation (full lines) and NOx reduction (dotted lines) for the $\mathrm{Ni}$-nitrate series comparing the nickel loading: a) following the reaction from 200 to 500 o $\mathrm{C}$; b) maximum values. 


\section{V.4. Conclusions}

Treatment of a saponite with nickel precursors successfully yields to layered solids with high surface area, microporosity and controlled nickel amounts. The characterization showed the presence of two types of nickel species with catalytic activities.

The resulting solids present similar behaviour than other nickel catalysts based on zeolitic supports for the selective catalytic reduction of nitrogen oxides, being possible to optimize the reaction rates choosing the adequate nickel precursor and the nickel content.

\section{V.5. References}

[1] A. Vaccari, Appl. Clay Sci. 14 (1999) 161-198.

[2] H.H. Murray, Appl. Clay Sci. 17 (2000) 207-221.

[3] Z. Ding, J.T. Kloprogge, R.L. Frost, G.Q. Lu, H.Y. Zhu, J. Porous Mater. 8 (2001) 273-293.

[4] E.M. Serwicka, K. Bahranowski, Catal. Today 90 (2004) 85-92.

[5] A. Gil, L.M. Gandia, M.A. Vicente, Catal. Rev. 42 (2000) 145-212.

[6] J.T. Kloprogge, J. Porous Mater. 5 (1998) 5-41.

[7] J.T. Kloprogge, L.V. Duong, R.L. Frost, Environ. Geol. 47 (2005) 967-981.

[8] R.S. Varma, Tetrahedron 58 (2002) 1235-1255.

[9] R.Q. Long, R.T. Yang, K.D. Zammit, J. Air Waste Manage. 50 (2000) 436-442; R.T. Yang, J. Cichanowicz, US Patent № 5.415.850, (1995).

[10] J.L. Valverde, A. de Lucas, P. Sánchez, E. Dorado, A. Romero, Appl. Catal. B 43 (2003) 43-56.

[11] A. de Lucas, J.L. Valverde, F. Dorado, A. Romero, I. Asencio, J. Mol. Catal. A 225 (2005) 47-58.

[12] R. Burch, Catal. Rev. 46 (2004) 271-333.

[13] X. She, M. Flytzani-Stephanopoulos, J. Catal. 237 (2006) 79-93.

[14] C. Belver, M.A. Vicente, A. Martínez-Arias, M. Fernández-García, Appl Catal. B 50 (2004) 227-234.

[15] G. Mata, R. Trujillano, M.A. Vicente, C. Belver, M. Fernández, S.A. Korili, A. Gil, Appl. Catal. A, in press, doi:10.1016/j.apcata.2007.04.021.

[16] J.L. Valverde, A. de Lucas, F. Dorado, A. Romero, P.B. García, J. Mol. Catal. A 230 (2005) 23-28. 
[17] S. Mendioroz, A.B. Martín-Rojo, F. Rivera, J.C. Martín, A. Bahamonde, M. Yates, Appl. Catal. B 64 (2006) 161-170.

[18] M. Meng, P. Lin, Y. Fu, Spectrosc. Lett. 34 (2001) 83-92.

[19] S.J. Zhang, L.D. Li, F.X. Zhang, B. Xue, N.J. Guan, Chinese J. Catal. 26 (2005) 929-932.

[20] R. Toranzo, M.A. Vicente, M.A. Bañares-Muñoz, Chem. Mater. 9 (1997) 18291836.

[21] N. Lahav, U. Shani, J. Shabtai, Clays Clay Miner. 26 (1978) 107-115.

[22] M. Che, O. Clause, Ch. Marcilly, Preparation of solid catalysts, Wiley-VCH, Weiheim, 1999.

[23] B. Cardoso, J. Pires, A.P. Carvalho, I. Kuzniarska-Biernacka, A.R. Silva, B. de Castro, C. Freire, Micropor. Mesopor. Mater. 86 (2005) 295-302.

[24] K.S.W. Sing, D.H. Everett, R.A.W. Haul, L. Moscou, R.A. Pierotti, J. Rouquerol, T. Siemieniewska, Pure Appl. Chem. 57 (1985) 603-619.

[25] F. Rouquerol, J. Rouquerol, K. Sing, Adsorption by powders and porous solids. Principles, methodology and applications, Academic Press, London, 1999.

[26] B. Casal, J. Merino, E. Ruiz-Hitzky, E. Gutiérrez, A. Aleares, Clay Miner. 32 (1997) 41-54.

[27] O. Prieto, M.A. Vicente, M.A. Bañares-Muñoz, J. Porous Mater. 6 (1999) 335344.

[28] K. Utchariyajit, E. Gulari, S. Wongkasemjit, Appl. Organomet. Chem. 20 (2005) 81-88.

[29] S.J. Zhang, L.D. Li, B. Xue, J.X. Chen, N.J. Guan, F.X. Zhang, Reac. Kinet. Catal. Lett. 89 (2006) 81-87.

[30] M. Jitianu, A. Jitianu, M. Zaharescu, D. Crisan, R. Marchitan, Vib. Spectrosc. 22 (2000) 75-86.

[31] B.I. Mosqueda-J iménez, A. Jentys, K. Seshan, J.A. Lercher, Appl. Catal. B 43 (2003) 105-115.

[32] N. Okazaki, Y. Katoh, Y. Shiina, A. Tada, M. I wamoto, Chem. Lett. 26 (1997) 889-890.

[33] M. Mihaylov, K. Hadjiivanov, D. Panayotov, Appl. Catal. B 51 (2004) 33-42.

[34] B.I. Mosqueda-Jiménez, A. Jentys, K. Seshan, J.A. Lercher, J. Catal. 218 (2003) 375-385.

[35] K. Shimizu, H. Maeshima, A. Satsuma, T. Hattori, Appl. Catal. B 18 (1998) 163-170. 


\section{Capítulo VI}

(Z)-Cyclooctene epoxidation and cyclohexane oxidation on $\mathrm{Ni}$ / alumina-pillared clay catalysts 



\section{Abstract}

This work reports the synthesis of $\mathrm{Ni}$-clay catalysts by impregnation of Al-pillared saponite with various $\mathrm{Ni}$ salts, using several $\mathrm{Ni}$ loadings. The catalysts were characterized by X-ray diffraction, infrared spectroscopy, thermogravimetric and differential thermal analysis, nitrogen adsorption, temperature-programmed reduction studies, and ultraviolet-visible spectroscopy. All the solids displayed good structural properties. FTIR spectroscopy confirmed the total removal of the anionic precursors after calcination of the solids at $500{ }^{\circ} \mathrm{C}$. The presence of $\mathrm{NiO}$ and $\mathrm{NiAl}_{2} \mathrm{O}_{4}$ in the nickel particles formed on the surface of the solids was demonstrated. Deconvolution of TPR curves allowed to identify three Ni reduction effects, their temperature and relative intensity depended on the preparation of each sample. The catalytic behaviour of the solids for (Z)-cyclooctene epoxidation and cyclohexane oxidation by either iodosylbenzene (a model oxidant) or hydrogen peroxide (a clean oxidant) was studied. In the case of the epoxidation reactions, there was $100 \%$ selectivity for the epoxide. As for cyclohexane oxidation, only cyclohexanone was formed, whereas no cyclohexanol was detected. The use of radical scavengers indicated that the high-valent oxo-nickel species is responsible for the oxygen transfer between the oxidant and the substrates.

Keywords: Alumina-pillared clay catalyst; Epoxidation; Nickel oxide; Catalytic oxidation

\section{VI.1. Introduction}

The controlled partial oxidation of alkanes, alkenes and aromatic hydrocarbons, usually involving metallic catalysts, is one of the most important technologies for the production of compounds with high added value from crude oil. Synthetic procedures usually consume high amounts of energy, produce waste contaminants and lead to low selectivity, sometimes giving rise to some undesired products. Thus, every effort has been made so that more efficient alternatives, such as soft oxidants and recyclable catalysts, can be obtained [1-5]. Although some enzymes can easily carry out hydrocarbon oxidation, reports on systems mimicking their biological selectivity are scarce [6]. Actually, the oxygen transfer from an oxidant to organic substrates by porphyrin systems and their related models has been well established, but the use of non-porphyrin transition metal materials, like the Factor 
430 system, as model systems for non-heme enzymatic reactions, has attracted the attention of various researchers [7]. In fact, Ni complexes have been frequently studied for this purpose. A further attractive alternative is the use of heterogeneous catalytic systems [8-12].

Epoxides are an important group of compounds obtained by catalytic oxidation, being strategic intermediates in the chemical industry. They can be converted into alcohols, polyethers and aldehydes, compounds that show widespread applications in the chemical, agrochemical and pharmaceutical industries $[13,14]$. Thus, epoxidation is undoubtedly one of the commonest transformations undergone by alkenes. The use of transition metal complexes as catalysts for this reaction has been a subject of great interest [15], oxidation being carried out by using several oxidants, such as oxygen, hydrogen peroxide, and tert-butylhydroperoxide, among others [16].

The mixture cyclohexanol/cyclohexanone, also called KA-oil (Ketone Alcohol), is very important for the manufacture of textile fibres, such as nylon- 6 and nylon-6.6. This mixture is usually obtained from the partial oxidation of cyclohexane by molecular oxygen catalyzed by soluble metal salts [17]. The industrial reactions take place at high temperatures and pressures, but only $4 \%$ cyclohexane conversion is obtained. Therefore, the search for catalysts that are efficient at mild temperatures is a great challenge for researchers and industries.

Hydrogen peroxide is considered a clean and environmentally friendly oxidant since it is safe and cheap, not to mention the fact that only water and oxygen are formed as by-products. In contrast, iodosylbenzene (PhIO) is considered to be environmentally hazardous, although it can give high oxidant conversion and is relatively inert in the absence of a catalyst. Various works in the literature have used the olefin/PhIO system, which makes this system useful for comparison purposes despite all its hazards. As emphasized by Koola and Kochi [18], the use of PhIO as oxidant is reminiscent of catalytic epoxidations induced by synthetic metalloporphyrins, where the use of this oxidant as oxygen donor was introduced in order to mimic the chemistry of cytochrome P-450. As recently stated by Groves [19], iodosylbenzene is a polymeric solid that does not contain a weak O-H bond, thus eliminating the occurrence of free radical chain reactions normally initiated by oxidants like alkyl hydroperoxides $\mathrm{R}-\mathrm{O}-\mathrm{O}-\mathrm{H}$. 
Pillared InterLayered Clays, PILCs, are an important family of porous solids. Their synthesis involves three main steps: i) polymerization of a cation, producing polycations; that is, positively-charged polymeric species, ii) cationic exchange between these polycations and the natural charge-compensating cations of smectitic clays, giving rise to the so-called interlayered clays, which are metastable, and iii) structure stabilization by calcination of the interlayered clays at moderate temperatures, which converts the polycations into oxi-hydroxidic phases named pillars. Pillars are stable, and their localization in the interlayer space maintains the layers separated by a long distance. The solids thus prepared, namely pillared interlayered clays, have a characteristic porous structure, conditioned by the number and size of the pillars in the interlayer region. At the same time, the clay layers avoid aggregation of the metallic oxide clusters during calcination, maintaining their dispersion. As observed, preparation of pillared clays implies nanometric modification of the material [20-27].

Nickel-based supported catalysts can be applied in a wide variety of reactions, including those involving hydrocarbons $[28,29]$. Considering that the peculiar porous structure of PILCs makes them attractive supports for catalyst preparation, in the present paper we report the preparation, characterization and catalytic oxidation behaviour of several nickel-saponite catalysts. The samples were prepared by impregnation of alumina-pillared saponite with several nickel precursors. The catalytic performance of these catalysts in (Z)-cyclooctene epoxidation, to form ( $Z$ )-cyloocteneoxide, and in the partial cyclohexane oxidation, to produce the KA-oil, by either iodosylbenzene or hydrogen peroxide as oxidant, is reported.

\section{VI.2. Experimental}

\section{VI.2.1. Raw materials, intercalation process and catalyst preparation}

The natural mineral clay used in this work was a saponite from Cabañas (Toledo, Spain), supplied by TOLSA (Madrid). The raw material was previously purified by dispersion-decantation, which led to the $2 \mu \mathrm{m}$ fraction [30].

The parent clay was intercalated with the aluminium polycation $\left[\mathrm{Al}_{13} \mathrm{O}_{4}(\mathrm{OH})_{24}\right.$ $\left.\left(\mathrm{H}_{2} \mathrm{O}\right)_{12}\right]^{7+}$, in short $\mathrm{Al}_{13}{ }^{7+}$, according to a previously described procedure [31]. An 
aluminium polycation solution was prepared by slow addition of a $\mathrm{NaOH}$ solution (Panreac, p.a.) to an $\mathrm{AlCl}_{3} \cdot 6 \mathrm{H}_{2} \mathrm{O}$ solution (Panreac, p.a.) under vigorous stirring, with a $\mathrm{OH}^{-} / \mathrm{Al}^{3+}$ molar ratio equal to $2.2[32,33]$. The resulting solution was left to age for $24 \mathrm{~h}$ at room temperature, under constant agitation. The solution was then added to a previously prepared clay suspension, at an $\mathrm{Al} / \mathrm{clay}$ ratio of $5 \mathrm{mmol} / \mathrm{g}$. The slurry was stirred for $24 \mathrm{~h}$ at room temperature, centrifuged and washed by dialysis with distilled water, dried in air at $70{ }^{\circ} \mathrm{C}$ for $16 \mathrm{~h}$, and then calcined at $500{ }^{\circ} \mathrm{C}$ for $2 \mathrm{~h}$, at a heating rate of $5^{\circ} \mathrm{C} / \mathrm{min}$, in order to obtain the aluminapillared saponite. The synthesized pillared saponite was designated Al-PILC. In some cases, the citation Al-intercalated saponite would refer to the dried sample obtained before calcination.

The synthesis of the nickel catalysts was carried out by incipient wetness impregnation of pillared clays [34]. The required amounts of $\mathrm{Ni}$ (II) nitrate, $\mathrm{Ni}$ (II) chloride and $\mathrm{Ni}$ (II) acetylacetonate salt solutions (Sigma-Aldrich) were slowly added to the clays, to give solids with a \% NiO content ranging from 1 to $6 \mathrm{wt}$. The resulting solids were dried and calcined by the same procedure reported above. The subsequent solids were designated $\mathrm{Ni}(\mathrm{nit}), \mathrm{NiCl}$ and $\mathrm{Ni}(\mathrm{acac})$, thus indicating the metal precursors $\mathrm{Ni}$ (II) nitrate, chloride and acetylacetonate, respectively, followed by the catalytst's nominal NiO load.

\section{VI.2.2. Characterization techniques}

X-ray diffraction (XRD) patterns of non-oriented powder samples, using $2 \theta$ ranging between $2^{\circ}$ and $70^{\circ}$ at a scanning rate of $2 \% / \mathrm{min}$, were obtained at $40 \mathrm{kV}$ and $30 \mathrm{~mA}$ on a Siemens D-500 diffractometer with Ni-filtered $\mathrm{Cu} \mathrm{K} \alpha$ radiation.

Elemental analysis of the solids was carried out by inductively coupled plasma optical emission spectroscopy (ICPS OES) at Activation Laboratories Ltd., Ancaster, Ontario, Canada.

FT-IR spectra were recorded in the $350-4000 \mathrm{~cm}^{-1}$ range in a Perkin-Elmer 1730 Infrared Fourier Transform Spectrometer, using the $\mathrm{KBr}$ pellet technique. About $1 \mathrm{mg}$ of the sample and $300 \mathrm{mg} \mathrm{KBr}$ were used in the preparation of the pellets. 
Thermal analysis was performed on the Perkin-Elmer analyzers TGA7 and DTA7, for thermogravimetric and differential thermal analysis, respectively. All measurements were carried out at a heating rate of $10^{\circ} \mathrm{C} / \mathrm{min}$ under a flow of air, Air Liquide, $99.999 \%$, of $20 \mathrm{~cm}^{3} / \mathrm{min}$.

Nitrogen, Air Liquide, $99.999 \%$, adsorption experiments were performed at $-196{ }^{\circ} \mathrm{C}$ on a static volumetric apparatus, Micromeritics ASAP 2010 adsorption analyzer. All the samples $(0.2 \mathrm{~g})$ had been previously degassed at room temperature for $6 \mathrm{~h}$, and then at $110^{\circ} \mathrm{C}$ for $2 \mathrm{~h}$, at a pressure lower than 6.67 Pa. The specific total surface area $\left(\mathrm{S}_{\mathrm{BET}}\right)$ was calculated using the BET equation from adsorption data in the relative pressure range between 0.05 and 0.20 , considering a nitrogen molecule cross-sectional area [35] of $0.162 \mathrm{~nm}^{2}$. The micropore volume was obtained by means of the t-method [35]. The total pore volume ( $\mathrm{Vp})$ of the solids was assessed from the amount of nitrogen adsorbed at a relative pressure of 0.99 , assuming that the density of the nitrogen condensed in the pores is equal to that of liquid nitrogen at $-196{ }^{\circ} \mathrm{C}, 0.81 \mathrm{~g} / \mathrm{cm}^{3}$ [35].

Temperature-Programmed Reduction (TPR) studies were carried out on a Micromeritics TPR/TPD 2900 instrument, from room temperature to $900{ }^{\circ} \mathrm{C}$, at a heating rate of $10{ }^{\circ} \mathrm{C} / \mathrm{min}$, under a total flow of $60 \mathrm{~cm}^{3} / \mathrm{min}, 5 \% \mathrm{H}_{2}$ in $\mathrm{Ar}$, Air Liquide. Only the solids calcined at $500{ }^{\circ} \mathrm{C}$ were studied by this technique, no pretreatments being needed. Water and other compounds that might have been formed during the metal reduction and precursor decomposition were retained by a molecular sieve trap. Hydrogen consumption was measured by a thermal conductivity detector (TCD), and calibration of the TCD signal was performed using $\mathrm{Ag}_{2} \mathrm{O}$ as external reference.

Diffuse Reflectance-UltraViolet-Visible (DR-UV-Vis) spectroscopy studies were recorded in the reflectance mode by using a Shimadzu spectrometer UV-2100, with an optical length of $0.4 \mathrm{~cm}$.

Scanning Electron Microscopy (SEM) of the materials was performed in a Digital Scanning Microscope DSM 960 Zeiss. The samples were coated with a thin gold layer using a Bio-Rad ES100 SEN coating system. 


\section{VI.2.3. Catalytic performance}

The catalytic behaviour of the catalysts in ( $Z$ )-cyclooctene epoxidation and cyclohexane oxidation by either iodosylbenzene or hydrogen peroxide was evaluated at $25^{\circ} \mathrm{C}$ and atmospheric pressure.

Iodosylbenzene (PhIO) was synthesized by hydrolysis of iodosylbenzenediacetate [36], dried under reduced pressure and kept at $5{ }^{\circ} \mathrm{C}$. The purity was periodically controlled by iodometric titration [37]. PhIO (22.7 $\mu \mathrm{mol})$ was added to a $4 \mathrm{~cm}^{3}$ vial sealed with a teflon-coated silicone septum containing one of the nickel catalysts $(10 \mathrm{mg})$, in a mixture of 1,2-dichloroethane $\left(1 \mathrm{~cm}^{3}\right)$; (Z)-cyclooctene (1.15 mmol, previously purified on alumina column) or cyclohexane $(1.39 \mathrm{mmol})$; and cyclohexanone or n-octanol as internal standard $\left(0.01 \mathrm{~cm}^{3}\right)$, respectively. The same procedure was carried out when hydrogen peroxide was employed as oxidant; the only difference was that hydrogen peroxide $70 \%(25 \mu \mathrm{L})$ was used instead of PhIO. At the end of the reaction, the solution was separated from the solid catalyst by centrifugation, the catalyst was washed several times with 1,2-dichloroethane, and the reaction products were analyzed by gas chromatography (Hewlett-Packard 6890). Yields were determined by comparison with pure compounds by means of calibration curves, and as indicated before using internal standards. A control of the reactions was carried out in the absence of nickel catalysts, with the natural saponite clay or the pillared saponite and the same reaction protocol described above.

\section{VI.3. Results and discussion}

\section{VI.3.1. Characterization of the catalysts}

Some of the solids used in the present work have been recently employed in the selective catalytic reduction of nitrogen oxide by propene [38]. Some characterization data concerning chemical analysis, X-ray diffraction, nitrogen adsorption, and DR-UV-Vis spectroscopy can be found in this reference. Here, we report only the data that are essential to make this work understandable as a whole, and we also extend the characterization by discussing FT-IR spectroscopy, thermal analyses, and TPR results. 
The process of intercalation-pillaring with aluminium polycations was successful. This was expected since this method is well standardized for the synthesis of pillared clays. The intercalated saponite showed a basal spacing of $18.2 \AA, 13.8 \AA$ in the natural clay, and this value slightly decreased to $17.8 \AA$ when the sample was calcined at $500{ }^{\circ} \mathrm{C}$ for the formation of the pillars. The XRD pattern of the pillared solid, used as support for the impregnated catalysts, is shown in Figure 1. Chemical analyses confirmed the successful intercalation of the clay, showing that the $\mathrm{Al}_{13}{ }^{7+}$ polycations substituted the original exchangeable cations of the clay, as noted by the increase in $\mathrm{Al}_{2} \mathrm{O}_{3}$ content and the decrease in $\mathrm{CaO}$ and, to a lesser extent, of $\mathrm{K}_{2} \mathrm{O}$ contents in the intercalated solid.

Because of the method used for impregnation, fixation of the targeted $\mathrm{NiO}$ amount and the subsequent relative variation in the amounts of the other elements was observed by chemical analyses of the solids. Impregnation caused a moderate structural alteration effect on the support, mainly by disordering the packing of the layers in the c-direction, leading to a decrease in the intensity of the basal reflection, which almost disappeared. This alteration became more evident when the solids were calcined at $500{ }^{\circ} \mathrm{C}$, a necessary step for the generation of the desired NiO final catalysts. The XRD patterns of the final catalysts from the series impregnated with $\mathrm{Ni}$-acac are shown in Figure 1, together with the diffractogram of the support. The loss of ordering in the c-axis was more evident and, in some cases, delaminated solids could be considered. The in-plane reflections of the clay, those independent of the c-packing, were not altered. Effects due to the precursors were observable only in the series obtained from acetylacetonate.

Figure 1. XRD diffractograms of the acac series of impregnated solids; the pattern of the support, Al-PILC solid, is shown as reference.

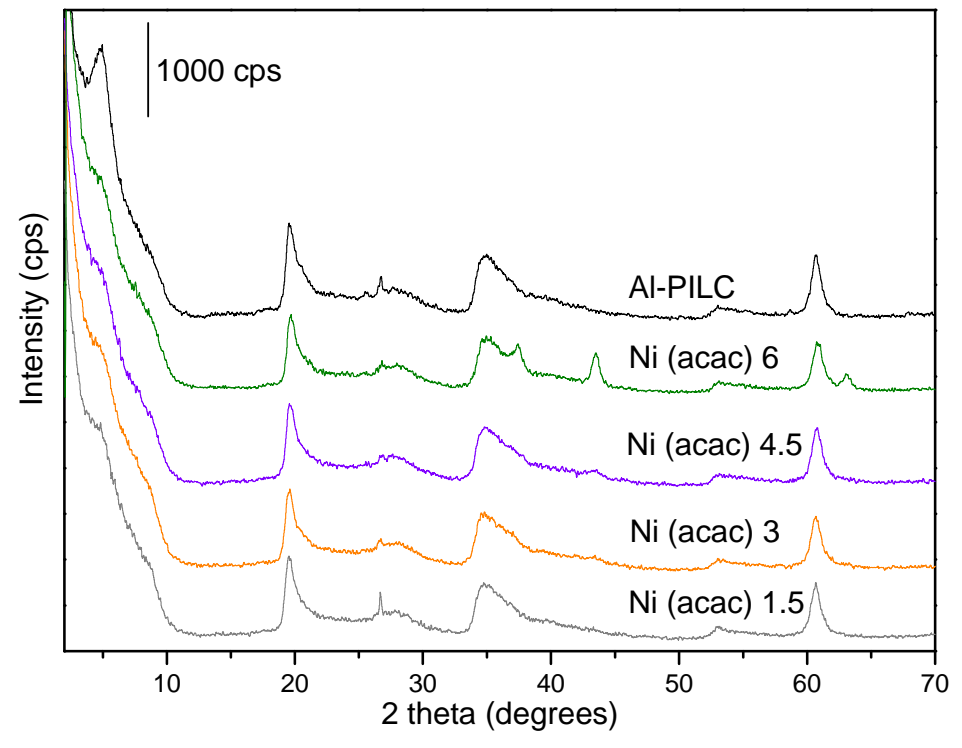


In the dried solids, diffractograms not shown, the crystallization of nickel acetylacetonate on the surface of the solid was clearly observed, while the calcination of these solids produced detectable crystallites of the NiO bunsenite phase, JCPDS pattern 4-0835 [39], mainly in the case of the solid with higher nickel content, sample $\mathrm{Ni}(\mathrm{acac}) 6$, diffractogram depicted in Figure 1 . The behaviour observed in the acetylacetonate series may be explained by the use of acetone as solvent in the preparation of the impregnated solids, and the easy crystallization of $\mathrm{Ni}$ acetylacetonate on the surface of the support when acetone evaporates. No crystalline phases were detected in the other solids, which displayed a high dispersion of the $\mathrm{Ni}$ species on the support surface. As $\mathrm{NiAl}_{2} \mathrm{O}_{4}$ is detected by DRUV-Vis spectroscopy (vide infra), it may be proposed that under calcination, the decomposition of the precursor mainly generates $\mathrm{NiO}$ particles. However, by interaction with the pillars and dissolution of small amounts of $\mathrm{Al}$ (III) cations, $\mathrm{NiAl}_{2} \mathrm{O}_{4}$, with a low degree of aggregation, may also be formed, leading to very small particles (except in the acac series) that may be mainly located at the extremes of the layer channels and on the external surface of the clay layers.

Infrared spectroscopy revealed incorporation of the precursors into the clay during the impregnation step, as well as removal of the anionic part of these precursors after calcination. Two significant examples can be seen in Figure 2. After impregnation, the effects of acetylacetonate and nitrate can be clearly observed in the dried solids of the corresponding series, while after calcination at $500{ }^{\circ} \mathrm{C}$, no effects due to these anions are noticed.

Figure 2. FT-IR spectra of two impregnated solids, before and after calcination at $500^{\circ} \mathrm{C}$.

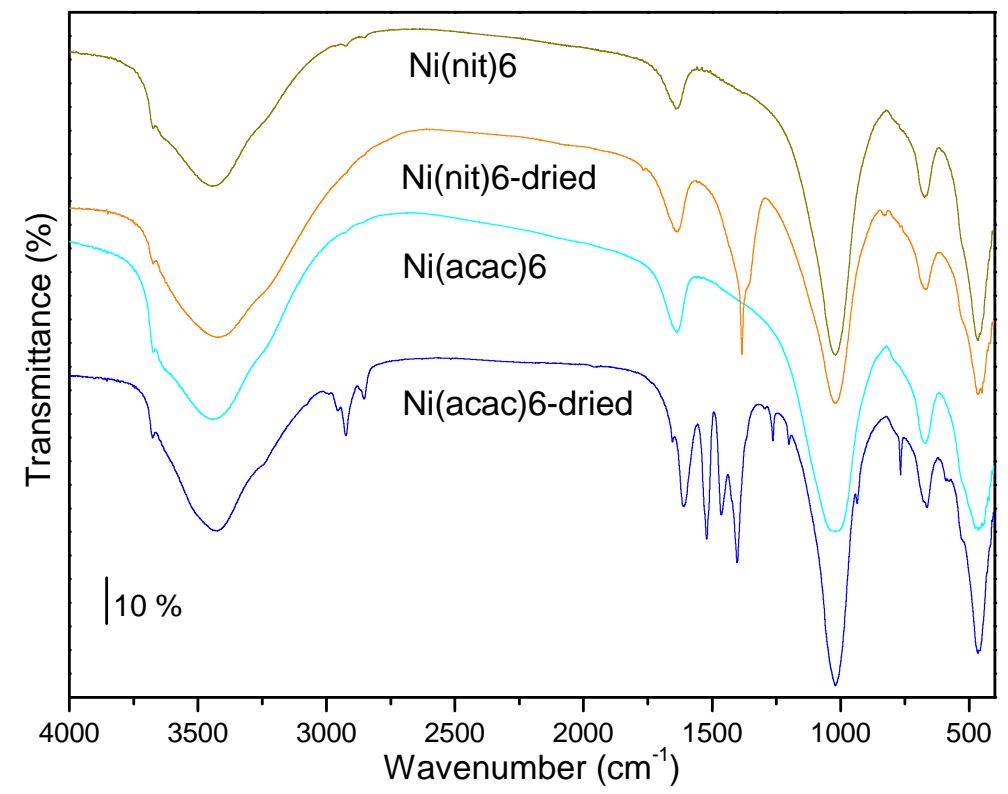




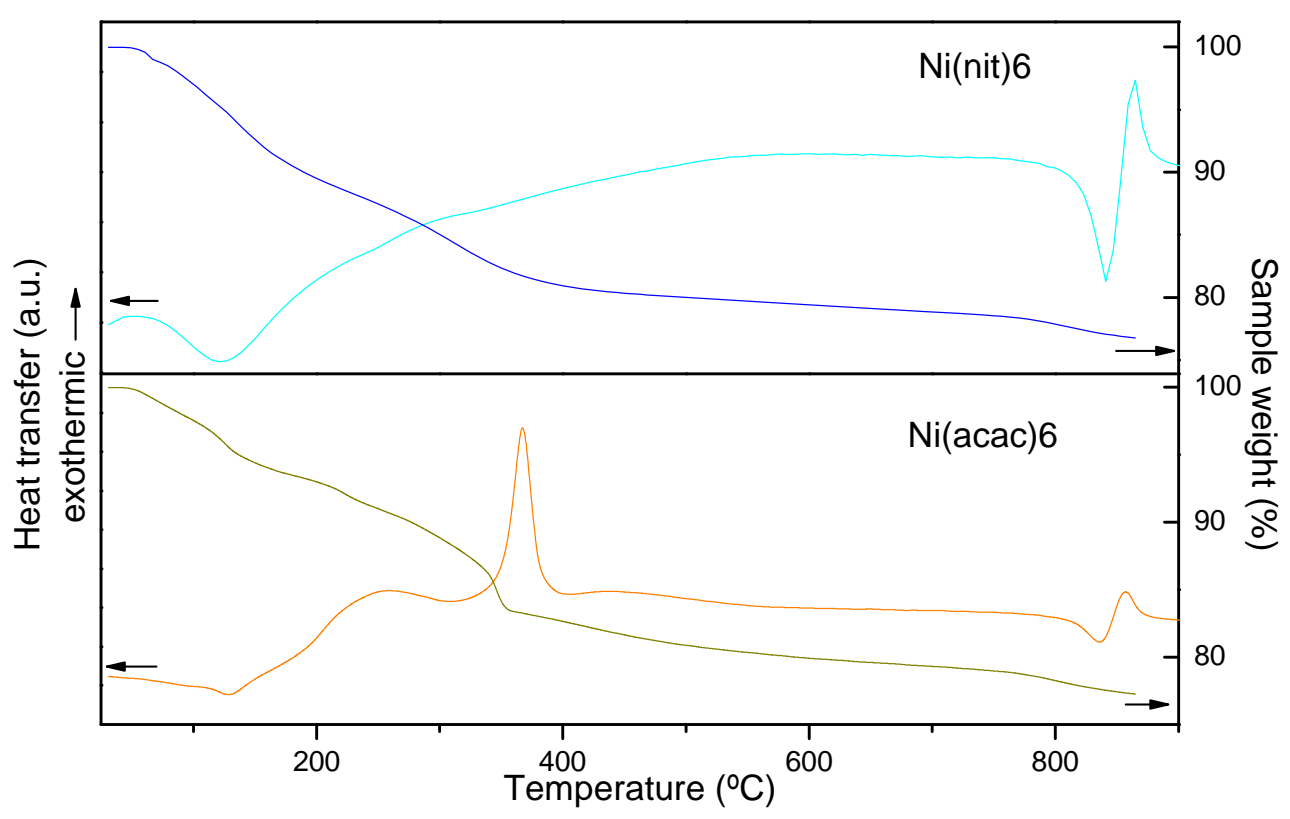

Figure 3. Thermal behaviour of two solids impregnated with Ni-nitrate and $\mathrm{Ni}$-acetylacetonate, respectively, under air atmosphere.

The decomposition of the precursors was checked by means of thermal analyses, which showed that (see Figure 3 ) the $\mathrm{Ni}$ acetylacetonate and the $\mathrm{Ni}$ nitrate supported over the clay decomposed at about $370{ }^{\circ} \mathrm{C}$ and $340{ }^{\circ} \mathrm{C}$, respectively, while decomposition of $\mathrm{Ni}$-chloride samples did not cause observable effects. Thus, calcination at this temperature produced the complete removal of the anions, and none of related species remained on the solids. The precursors are thus expected to be transformed into NiO-like phases after calcination, and FT-IR spectra confirmed the absence of residual components of these molecules in the calcined solids.

Before calcination, the impregnated solids had low specific surface areas, lower than $10 \mathrm{~m}^{2} / \mathrm{g}$. This is because of the occlusion of intra- and interparticular porosity by the impregnating species. Both natural and pillared saponites have a high surface area, 191 and $230 \mathrm{~m}^{2} / \mathrm{g}$, respectively. It is remarkable that the samples obtained from $\mathrm{Ni}$ acetylacetonate displayed surface areas of about $180 \mathrm{~m}^{2} / \mathrm{g}$, which are clearly larger than those presented by the $\mathrm{Ni}$ chloride, and the Ni nitrate series, between 102 and $159 \mathrm{~m}^{2} / \mathrm{g}$ and between 146 and $169 \mathrm{~m}^{2} / \mathrm{g}$, respectively. In all the cases, the microporous surface area was about $70 \%$ of the BET value. Besides, the surface area was virtually constant in the four samples belonging to the $\mathrm{Ni}$ acetylacetonate series, independent of the amount of precursor incorporated into 
the support, while in the other two series the surface area decreased with increasing metal loading, which agrees with the blocking of porosity by the $\mathrm{Ni}$ species. The high surface area of the samples belonging to the $\mathrm{Ni}$ acetylacetonate series may be related to the galleries opened by the $\mathrm{CO}_{2}$ produced in the combustion of the organic anions. Also, the total pore volume displayed a similar behaviour, being clearly higher in the acetylacetonate series, close to $0.17 \mathrm{~cm}^{3} / \mathrm{g}$, than in the other series, between 0.10 and $0.15 \mathrm{~cm}^{3} / \mathrm{g}$.

The temperature-programmed reduction profiles of some of the impregnated catalysts are shown in Figure 4A, together with the profile obtained for commercial $\mathrm{NiO}$ (Sigma-Aldrich, >99.999\%). NiO displayed a sharp reduction effect centred at $295^{\circ} \mathrm{C}$, and hydrogen consumption perfectly matched the expected $\mathrm{H}_{2} / \mathrm{Ni}$ ratio. The TPR profile of the support (pillared saponite) only showed a small effect at high temperature, corresponding to the reduction of the low amount of iron present in its octahedral sheet. Although the reduction profile of the support is not given, this effect could be clearly seen in the profiles of the supported catalysts, close to $760^{\circ} \mathrm{C}$.

The TPR profiles of the impregnated solids revealed up to four effects, the commented reduction of clay $\mathrm{Fe}$ (III) and three effects that can be assigned to the reduction of $\mathrm{Ni}$ (II) cations. In fact, the deconvolution of the Ni-reduction effect into three components was in excellent agreement with the experimental curve; the deconvolution of one of the curves is given in Figure $4 B$, and the results of this deconvolution are summarized in Table 1 . The second and third effects were centred at ca. 490 and $675{ }^{\circ} \mathrm{C}$, respectively, at very close temperatures for the three samples (ca. $25-30{ }^{\circ} \mathrm{C}$ higher in the $\mathrm{Ni}$ (acac)4.5 sample), while the first effect was centred at $338{ }^{\circ} \mathrm{C}$ for the $\mathrm{Ni}(\mathrm{acac}) 3$ sample, and at a significantly higher temperature for the two other samples ( 369 and $366^{\circ} \mathrm{C}$ ). The relative area of each effect was even more interesting. It is noteworthy that the first effect was much more intense in the $\mathrm{Ni}(\mathrm{acac}) 6$ sample (ca. 34\%) than in the other samples ( 7 and $13 \%)$. In other words, when the Ni loading is low, the effects at high temperature are predominant, but the higher the $\mathrm{Ni}$ loading, the more intense the first effect. Thus, it may be proposed that the $\mathrm{NiO}$ particles are clearly more similar to the bulk oxide when the metal loading is increased, facilitating its reduction. On the other 
hand, the very dispersed particles formed at low Ni loadings have lower reducibility, requiring higher temperatures for this process. For all the solids, $\mathrm{H}_{2} / \mathrm{Ni}$ ratios close to the theoretical value from the $\mathrm{Ni}(\mathrm{II}) \rightarrow \mathrm{Ni}(0)$ reduction were found.
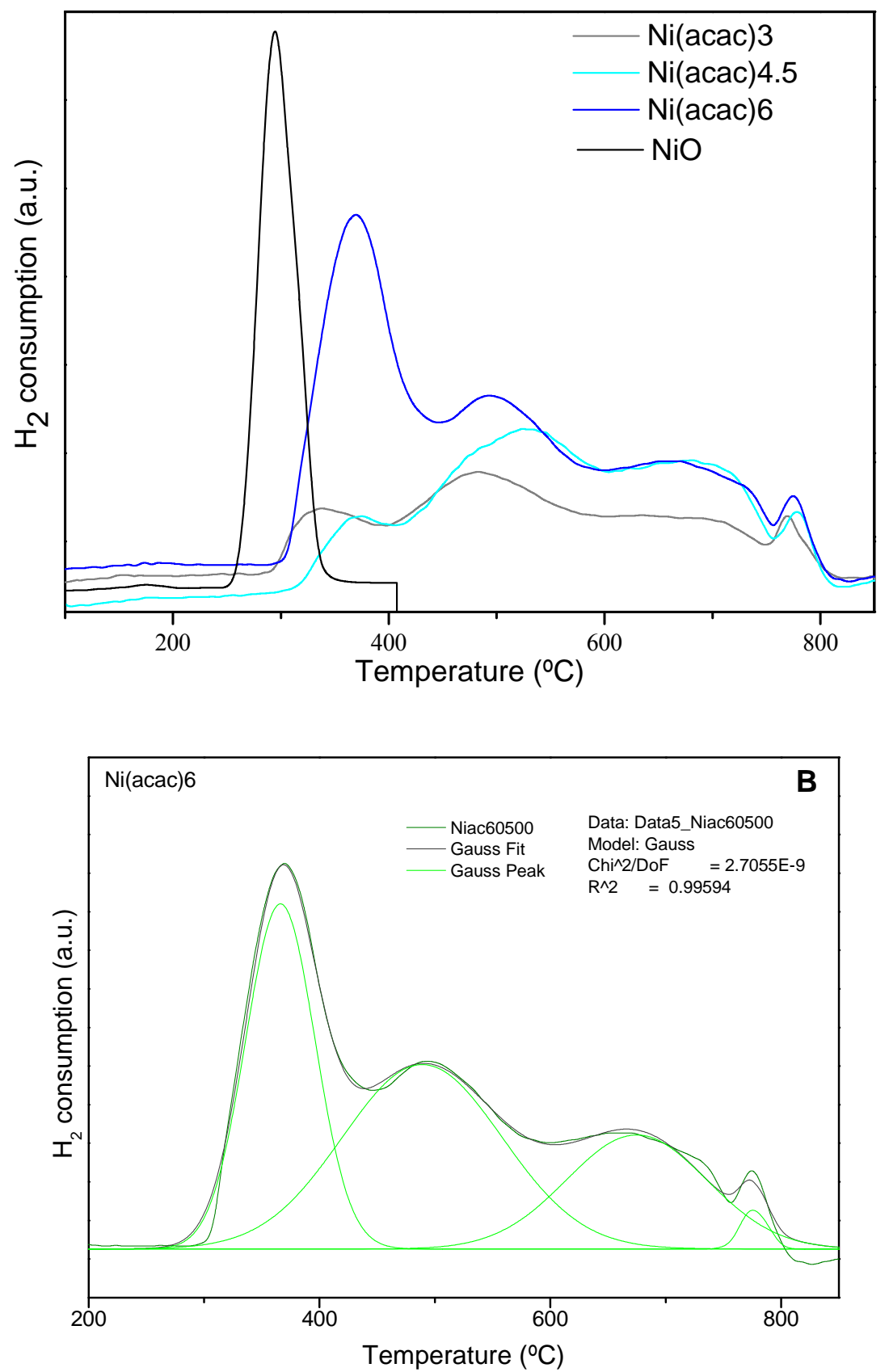

Figure 4. A. TPR curves of three solids impregnated with Ni acetylacetonate; the curve of bulk $\mathrm{NiO}$ is included as reference, its intensity being multiplied by 0.5 .

B. Deconvolution of the reduction curve of the $\mathrm{Ni}(\mathrm{acac}) 6$ sample. 


\begin{tabular}{|c|c|c|c|}
\hline & $\mathrm{Ni}($ acac $) 3$ & $\mathrm{Ni}($ acac $) 4.5$ & $\mathrm{Ni}($ acac $) 6$ \\
\hline \multicolumn{4}{|l|}{ Peak 1} \\
\hline Temperature & 339 & 369 & 366 \\
\hline Relative area & 13.4 & 7.1 & 33.7 \\
\hline \multicolumn{4}{|l|}{ Peak 2} \\
\hline Temperature & 484 & 521 & 489 \\
\hline Relative area & 53.8 & 57.0 & 41.3 \\
\hline \multicolumn{4}{|l|}{ Peak 3} \\
\hline Temperature & 671 & 695 & 675 \\
\hline Relative area & 32.8 & 35.9 & 25.0 \\
\hline
\end{tabular}

DR-UV-Vis spectroscopy of the impregnated solids showed that the nickel species are octahedral $\mathrm{Ni}(\mathrm{II})$ cations in the $\mathrm{NiO}$ phase, $\mathrm{NaCl}$ structure $\left({ }^{3} \mathrm{~A}_{2 \mathrm{~g}} \rightarrow{ }^{1} \mathrm{~T}_{1 \mathrm{~g}}\right.$ (D) transition, ca. $350 \mathrm{~nm})$, tetrahedral $\mathrm{Ni}(\mathrm{II})$ cations in the $\mathrm{NiAl}_{2} \mathrm{O}_{4}$ spinel $\left({ }^{3} \mathrm{~T}_{1} \rightarrow\right.$ ${ }^{1} \mathrm{~T}_{2}$ and ${ }^{3} \mathrm{~T}_{1} \rightarrow{ }^{3} \mathrm{~T}_{1}(\mathrm{P})$ transitions, 590-650 nm), and octahedral $\mathrm{Ni}(\mathrm{II})$ in the alumina lattice of the $\mathrm{NiAl}_{2} \mathrm{O}_{4}$ spinel $\left({ }^{3} \mathrm{~A}_{2 g} \rightarrow{ }^{1} \mathrm{~T}_{1 \mathrm{~g}}(\mathrm{P})\right.$ transition, $\left.390 \mathrm{~nm}\right)$. This spinel was probably formed via interaction of the Ni precursor with the Al from the pillars [4042]. In the $\mathrm{Ni}$ (acac) series, a band due to octahedral $\mathrm{Ni}$ (II) in $\mathrm{NiO}\left({ }^{3} \mathrm{~A}_{2 g} \rightarrow{ }^{3} \mathrm{~T}_{1 \mathrm{~g}}(\mathrm{~F})\right.$ transition, $715 \mathrm{~nm}$ ), not observed in the other solids, was detected [43]. The detailed comparison of the spectra of the solids suggests that similar Ni species are formed in all the solids after the calcination process, independent of the precursor used for impregnation, and that $\mathrm{Ni}$ (II) acetylacetonate is the precursor that gives rise to larger amounts of $\mathrm{NiO}$ and $\mathrm{NiAl}_{2} \mathrm{O}_{4}$ species.

SEM micrographs of natural saponite and of two $\mathrm{Ni}$-containing solids are given in Figure 5. In spite of the treatment processes (pillaring, impregnation, calcination), the morphology of the samples is not very different. Particles of impregnated solids seem to be more compact, less diffuse, probably because of the two calcinations suffered during the treatments. In any case, they maintained the spongy aspect of the natural sample, showing low degradation. 


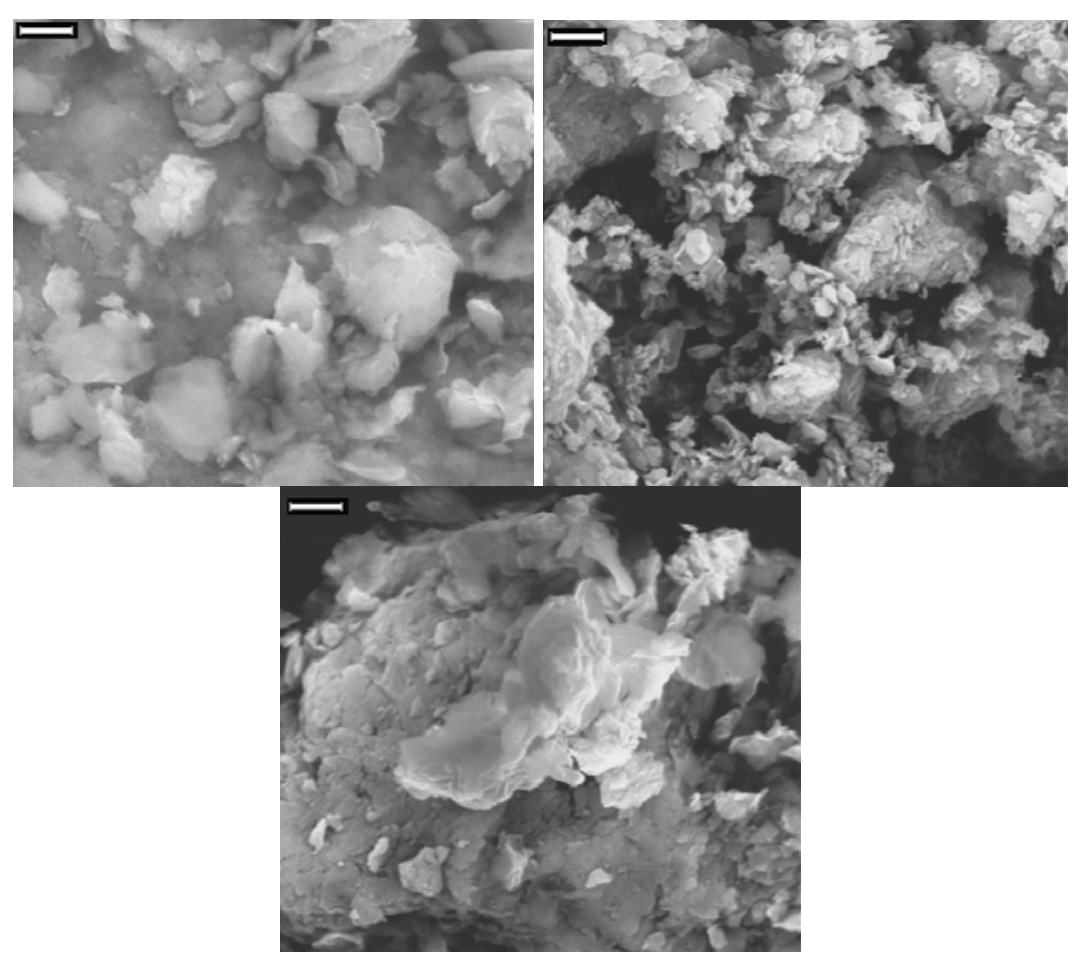

Figure 5. SEM micrographs of natural saponite and samples $\mathrm{Ni}(\mathrm{acac}) 6$ and $\mathrm{Ni}(\mathrm{nit}) 6$ (from left to right). In all cases, the segment has a length of 2.0 micrometers.

\section{VI.3.2. Catalytic performance}

In order to check the catalytic performance of the catalysts prepared in this work, (Z)-cyclooctene was oxidized by PhIO in the presence of the Ni-clay at room temperature and pressure. (Z)-cyclooctene oxidation produces (Z)cycloocteneoxide as the main reaction product by the following reaction scheme: Cyclooctene + Iodosylbenzene $\rightarrow$ Cycloocteneoxide + Iodobenzene. The overall oxidant performance was achieved from the iodobenzene (PhI) yield in all the reactions. Blank tests were carried out in the absence of nickel-solid and in the absence of PhIO oxidant, and they did not lead to any product yields. When Ni-clay catalysts were employed, though, the oxidation reaction was effectively catalyzed in all cases, see Figures 6.A, B. 

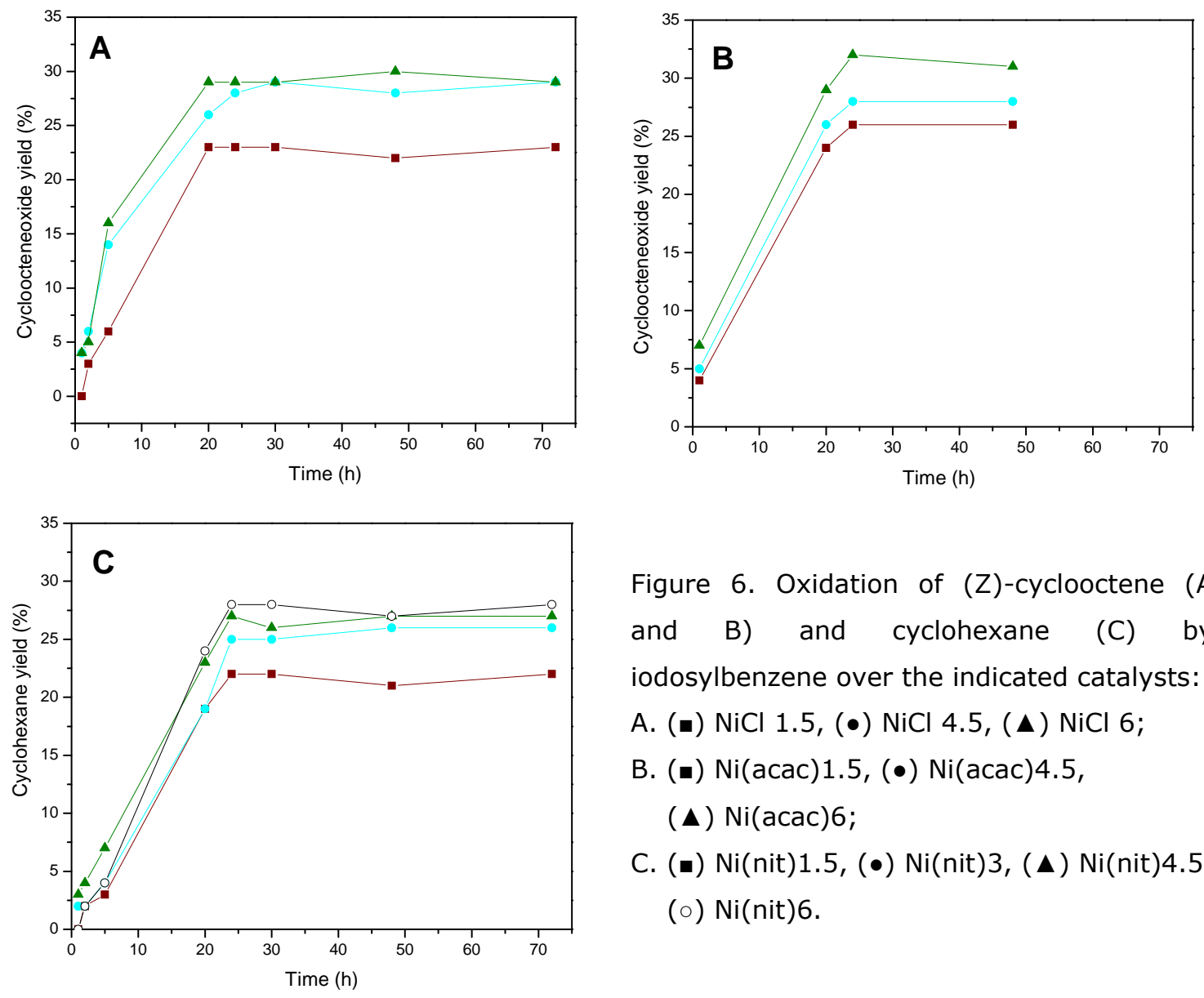

Figure 6. Oxidation of ( $Z$ )-cyclooctene (A and $B$ ) and cyclohexane (C) by iodosylbenzene over the indicated catalysts:

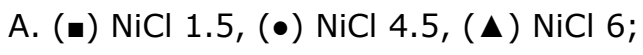

B. (घ) $\mathrm{Ni}($ acac) 1.5, (•) $\mathrm{Ni}($ acac $) 4.5$,

(A) $\mathrm{Ni}($ acac)6;

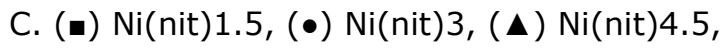
(o) $\mathrm{Ni}($ nit) 6 .

The UV-Vis spectra of the supernatant solutions obtained after the oxidation reactions showed that there was no metal leaching from the support in any of the studied conditions, since the typical $\mathrm{Ni}(\mathrm{II}) \mathrm{d}-\mathrm{d}$ bands were not detected in the solution. The solid was filtered off the reaction mixture, and an additional amount of oxidant was added to the resulting supernatant, and the oxidation reaction was allowed to proceed under the same initial conditions for another $24 \mathrm{~h}$. After this period, no products were detected, indicating that the supported solids played an essential role in the reaction. Another evidence of the heterogeneous character of the oxidation catalysis was obtained by removing the solid catalyst from the reaction solution at the reaction time of $24 \mathrm{~h}$ and further continuing the reaction by using the filtrate. In this case, it was observed that epoxide formation stopped after separation of the solid catalyst. Cyclooctene oxide was the only product in all reactions.

Because $\mathrm{Fe}(\mathrm{III})$ present in the saponite structure can also catalyze the oxidation, as observed when montmorillonite clay was used as catalyst [44], new 156 
blank tests were carried out using natural and pillared saponite as catalysts. This way, the influence of the pillaring step, which led to an increase in the aluminium content and in the surface area and porosity with respect to the natural clay, was also verified. The use of natural saponite led to only $8 \%$ epoxide yield, while $18 \%$ epoxide yield was obtained with the pillared clay. The catalytic behaviour of the supports was much lower than that of the Ni-clay materials, for which $32 \%$ yields were obtained. In general, epoxide yields depended on the precursor used for catalyst preparation. In fact, only slight variations in the yields were observed for close $\mathrm{Ni}$ amounts (29-32 \% of cyloocteneoxide), with the $\mathrm{Ni}(\mathrm{acac})$ series showing the best behaviour. In each series, the product yields increased with increasing amount of $\mathrm{Ni}$ incorporated into the solids. This could be an indication of the role played by nickel as active site and is in agreement with the fact that the active phases are very similar for all the solids after calcination at $500{ }^{\circ} \mathrm{C}$, independent of the precursor. This fact can be explained on the basis of the availability of the active sites on the surface of the materials, which favours the approach of the substrate $(Z)$-cyclooctene and the diffusion of the product cis-cycloocteneoxide from the heterogeneous catalyst to bulk solution.

As (Z)-cyclooctene epoxidation reactions were not carried out under inert atmosphere; oxygen could have reacted with the alkenes in the presence of metal ions, thus generating free radicals. We tried to distinguish between a radical mechanism and one involving high-valent metal-oxo species by carrying out ( $Z$ )cyclooctene epoxidation in the presence of a scavenger, the tert-butyl alcohol radical $[45,46]$. This radical scavenger did not diminish the product yields, so the involvement of the radical species as the epoxidizing agent was discarded. To confirm this hypothesis, another experiment was carried out in the presence of a different radical scavenger, namely hydroquinone, and again the yield did not decrease, thus allowing for the complete exclusion of the participation of the radical mechanism in the reaction.

It has been reported that insensitivity to the radical scavenger is understandable considering that the free radicals are not viable intermediates in a catalytic cycle involving nickel-oxo species [28]. Ferreira et al. [47] observed the competition between the radical and metal-oxo active species in Ni-salen systems due to the presence of oxygen. Therefore, it can be proposed that epoxidation over our $\mathrm{Ni}$ supported catalysts should occur in two steps, via a concerted mechanism. 
First, the active species $\mathrm{Ni}^{\mathrm{iV}}=\mathrm{O}$ are formed via a two-electron oxidation of the $\mathrm{Ni}^{2+}$ ions by PhIO. Then, the $\mathrm{Ni}^{\mathrm{IV}}=\mathrm{O}$ species transfer the oxygen atom to the $\mathrm{C}=\mathrm{C}$ bond of the substrate, leading to the formation of the epoxide.

Results obtained for cyclohexane oxidation reactions using the Ni-clay catalysts are shown in Figure 6.C. Cyclohexanone was the only product detected in the reaction. As in the case of $(Z)$-cyclooctene epoxidation, no nickel ion leaching from the support occurred. We found that nickel plays an essential role in mediating oxygen transfer from the oxidant to the substrate, since natural saponite led to a cyclohexane conversion of only $4 \%$. The yield obtained for this reaction with the $\mathrm{Ni}$-clay catalysts is significantly higher than those reported in the literature using other metallic complexes [48].

It has been reported that cyclohexanol is readily converted into cyclohexanone in the presence of transition metal ions as catalysts [49]. The mechanism for this catalytic reaction was proposed by Inchley et al. [50] using porphyrins as catalysts. It has also been frequently observed that cyclohexane may be directly oxidized to cyclohexanone, or be oxidized to cyclohexanol in a first step, followed by the subsequent oxidation of cyclohexanol to cyclohexanone. In order to differentiate between both possible routes, we carried out the reaction using cyclohexanol as substrate, and no ketone formation was observed. This result shows that oxidation does not occur through a two-step route and strongly suggests the direct cyclohexane oxidation to cyclohexanone. This result is also in accordance with the fact that no alcohol formation was observed when cyclohexane was used as substrate.

If the ketone is the major product in the oxidation of cycloalkanes catalyzed by metallocomplexes, it is accepted that a radical mechanism or a metal-oxo species mechanism is involved. In order to test whether the radical route is generating catalytically active species, the reaction was repeated in the presence of the radical scavengers, tert-butyl alcohol or hydroquinone. No modification of the results was observed, suggesting that a nickel high-valent species are a possible active intermediate in these reactions [47]. This also agrees with the high selectivity observed for ketone formation; typically, the radical mechanism decreases the selectivity and favors a mixture of products. 
Recently, Nagataki et al. [51], using the Ni-mCPBA complex, mCPBA being meta-chloroperbenzoic acid, in the homogeneous oxidation of cyclohexane, obtained a mixture of alcohol and ketone, but with high selectivity for cyclohexanol. The selectivity for the alcohol was attributed to the high reactivity of the metal-oxo species [52]. On the basis of the remarkable differences in the results obtained in homogeneous and heterogeneous catalysis, it is reasonable to think that the saponite support can be directly acting on the catalyst selectivity, exerting a similar effect to that wielded by several ligands in homogeneous catalysis. For example, the acid character of the clay enhances the formation of the metal-oxo active species that favours the production of the most polar product, the ketone.

The catalytic efficiency and stability of Ni-clays in the oxidation of Z-cyclooctene was also evaluated from hydrogen peroxide as oxygen donor at room temperature. In the last years, an increasing attention has been paid to the use of clean technologies employing hydrogen peroxide as oxidant. This compound is a promising oxygen donor because it is relatively cheap and readily available, and it contains a high level of active oxygen. Moreover, only water and oxygen are generated from this oxidant, which should help to reduce the costs related to industrial wastewater treatment. In fact, the reaction scheme is: Cyclooctene + Hydrogen peroxide $\rightarrow$ Cycloocteneoxide + Water. The large-scale use of hydrogen peroxide strongly depends on the development of appropriate catalysts, because the presence of water as by-product may lead to catalyst deactivation, and further reactions may take place between the products and the initial reagents that remain in the reaction medium.

The epoxide yields over the several catalysts are presented in Figure 7. All the nickel-saponite catalysts efficiently catalyzed Z-cyclooctene epoxidation by $\mathrm{H}_{2} \mathrm{O}_{2}$. Control reaction carried out using the natural saponite led to an epoxide yield of only $6 \%$. Again, this gives new evidence that the nickel ions are essential for the formation of the active species responsible for the oxygen transfer from the oxidant to the substrate. As in the case of $(Z)$-cyclooctene epoxidation by PhIO, no nickel leaching from the support was observed.

Comparison of the performances of the catalysts obtained with different precursors showed that $\mathrm{Ni}(\mathrm{nit})$ and $\mathrm{Ni}(\mathrm{acac})$ systems led to higher yields, while lower yields were obtained over the $\mathrm{Ni}(\mathrm{Cl})$ series. 

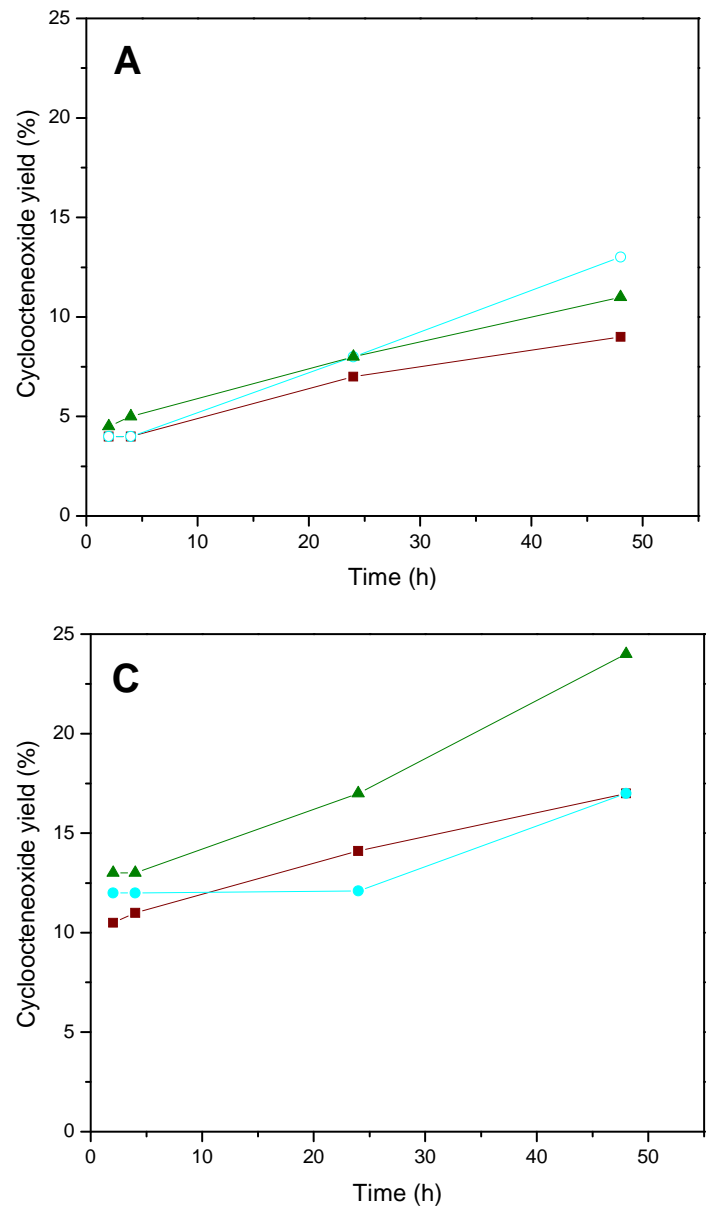

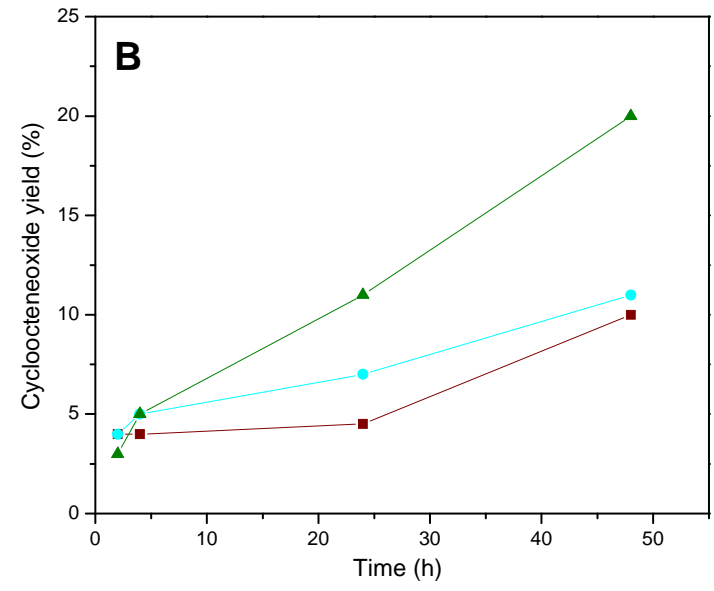

Figure 7. Oxidation of (Z)-cyclooctene by hydrogen peroxide over the indicated catalysts:

A. (घ) NiCl1.5, (०) NiCl4.5, (ム) NiCl6;

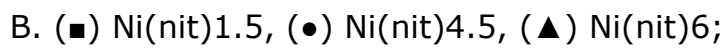

C. (•) $\mathrm{Ni}($ acac) 1.5, (•) $\mathrm{Ni}($ acac $) 4.5$,

(A) $\mathrm{Ni}($ acac $) 6$.

Note that the scales of both the yield and the time are different from those given in Figure 6.

The possible presence of chloride anions in the clays plays an important role in the catalytic process when hydrogen peroxide is employed as oxidant. After calcination, chloride anions may remain in the matrix, while the anionic part of the other precursors is completely removed. The more basic character of oxo anions in the NiO crystallites, compared with chloride anions, facilitates hydrogen peroxide deprotonation, thereby facilitating coordination of the $\mathrm{OOH}$ to the nickel center. Besides that, the acid character of the metal decreases in the presence of oxygen, favoring cleavage of the $\mathrm{O}-\mathrm{O}$ group and making formation of the $\mathrm{Ni}$-oxo species easier than in the case when chloride ions are present. Moreover, the surface area of the $\mathrm{Ni}-\mathrm{Cl}$ series solids is significantly lower compared with the other series, so accessibility to the active $\mathrm{NiO}$ crystallites may also influence the catalytic activity of these solids. It is known that, in the case of iodosylbenzene, which is a polymeric solid that does not contain a weak $\mathrm{O}-\mathrm{H}$ bond, there is no need for the occurrence of free radical chain reactions normally initiated by oxidants like alkyl hydroperoxides $\mathrm{R}-\mathrm{O}-\mathrm{O}-\mathrm{H}$ [19]. Therefore, hydrocarbon oxidation by PhIO does not depend on the $\mathrm{O}-\mathrm{O}$ bond cleavage as in the case of hydrogen peroxide, and the catalytic 160 
performance in the former case may not be influenced by the possible presence of chloride anions.
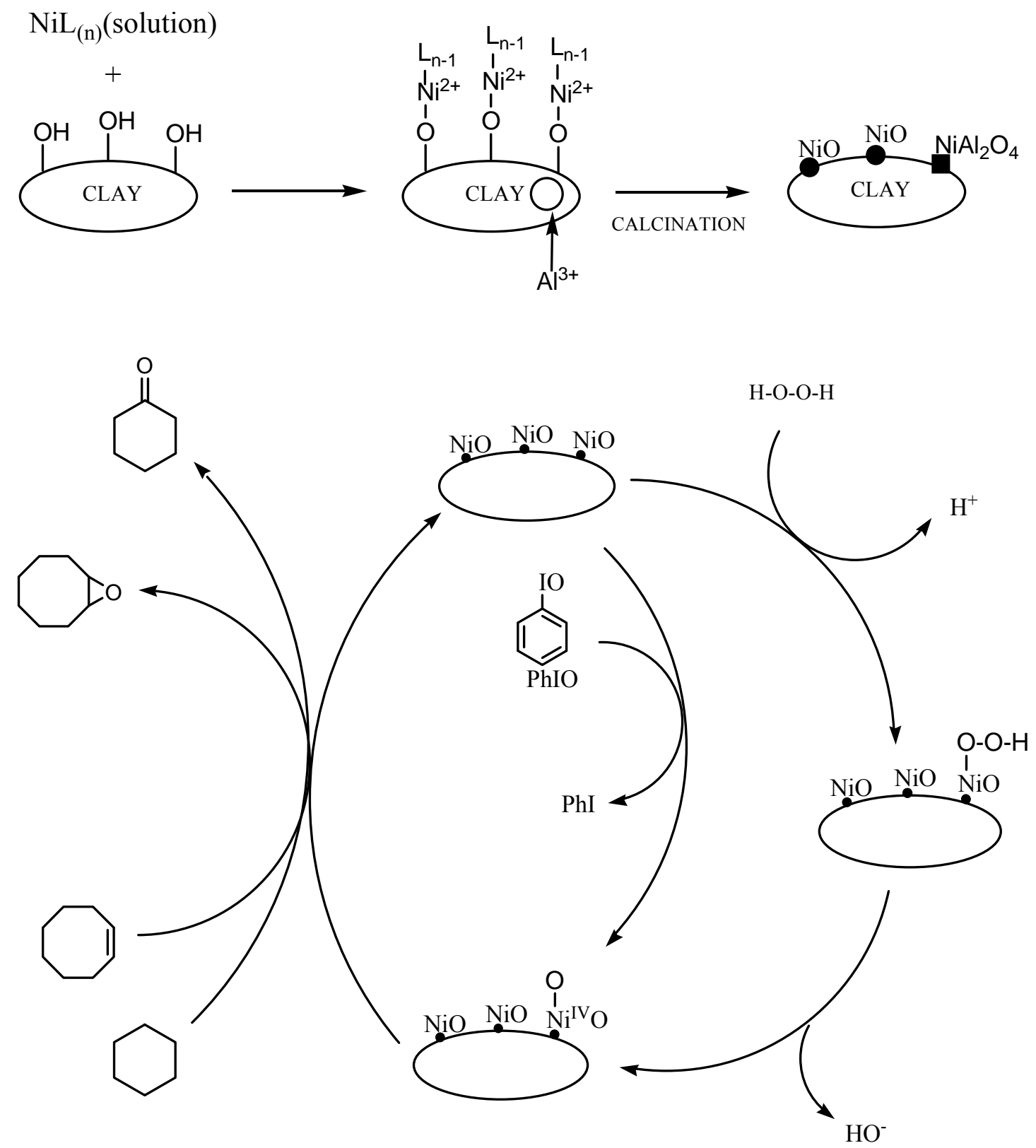

Scheme I. Mechanism proposed for the oxidation of cyclooctene and cyclohexane with iodosylbenzene over $\mathrm{Ni}$-clay catalysts.

No products were detected in the cyclohexane oxidation by $\mathrm{H}_{2} \mathrm{O}_{2}$ catalyzed by $\mathrm{Ni}$-clay. This result is not surprising since it is frequently reported that different metallocomplexes and inorganic solids containing metal ions, like metal-clays, present a catalyst-like activity and catalyze hydrogen peroxide disproportionation [53]. Because cyclohexane is a more inert substrate than olefins, the disproportionation reaction can occur before the generation of the catalytic species. 
In the case of olefin oxidation, hydrogen peroxide disproportionation can also occur, but because this substrate is more reactive than cyclohexane, the epoxidation reaction can be energetically favoured and the epoxidation reaction rapidly takes place. A mechanism for these reactions is proposed in Scheme I.

Additional cyclohexane oxidation experiments were carried out using mCPBA. There was a high selectivity for cyclohexanol, with the formation of small quantities of cyclohexanone. This selectivity is also attributed to the metal-oxo species, as previously observed by Costas et al. [52]. Hydrogen peroxide is more reactive than mCPBA, so its decomposition is easier in the support used herein. The versatile nature of the catalysts prepared in this work gives strong evidence that metal-oxo are the active species responsible for oxygen atom transfer from the oxidant to the studied substrates.

Although the results presented here strongly suggest the presence of a highvalent metal-oxo active species, a homolytic peroxide cleavage leading to radical species may also occur when hydrogen peroxide is used as oxidant. These radical species may normally result in considerable selectivity loss. In this study, only the formation of the epoxide was detected. In order to evaluate the existence of radical species, we repeated the reaction in the presence of tert-butyl alcohol, a radical scavenger; the cyloocteneoxide yield was the same as that obtained in the absence of the trap, thus confirming the absence of radical species in this system.

To confirm the non-radical character of the reaction, oxidation was carried out using the free-radical initiator tert-butyl hydroperoxide, TBHP, and no products were detected. This is in contrast with the results reported by Raja et al., who employed Fe(III), Co(III) and Mn(III) framework-substituted aluminium phosphate (M-AIPO) as catalysts. These authors found that cyclohexane oxidation proceeded by a free-radical mechanism, with the formation of the radical cyclohexyl hydroperoxide (cHHP) during the initial stages of the reaction, which subsequently decomposed to cyclohexanol and cyclohexanone [54]. It is clear that such a mechanism does not govern the reaction in the case of our Ni-clay catalysts. On the other hand, limonene epoxidation was carried out under the same conditions used for cyclooctene, and although conversion of limonene was only $2 \%$ after $48 \mathrm{~h}$ of reaction, epoxide was the only product, which once again confirms the absence of a radical mechanism in our systems [55]. 


\section{VI.4. Conclusions}

Treatment of intercalated clays with various nickel precursors successfully yields layered solids with high surface area, microporosity and controlled nickel amounts. Catalytic tests performed over these catalysts revealed that this methodology enables the design of efficient and selective heterogeneous catalysts, optimizing the use of the Ni-clay catalysts. The versatile nature of the catalysts for the oxidation of the alkene (Z)-cyclooctene and the cyclic hydrocarbon cyclohexane, using iodosylbenzene or hydrogen peroxide as oxygen donors, together with the data obtained in the presence of radical scavengers, gives strong evidence that highvalent oxo-nickel species is the active species responsible for oxygen transfer from the oxidant to the substrates, as in the case of enzymatic biological systems. A mechanism for the catalytic process has been proposed.

The obtained results demonstrated that the epoxidation of cyclooctene and the oxidation of cyclohexane occur with high selectivity for both substrates. The acid character of the clay materials enhances the formation of the metal-oxo active species that favours the production of the more polar products, the ketone. The results obtained in this work are very promising, since the formation of only one product resembles the results from biological system, which saves the necessity of separation steps, opening industrial perspectives for these systems.

\section{VI.5. References}

[1] W.R. Sanderson, Pure Appl. Chem. 72 (2000) 1289.

[2] B.S. Lane, M. Vogt, V.J. DeRose, K. Burgess, J. Am. Chem. Soc. 124 (2002) 11946.

[3] Z.L. Lu, E. Lindner, H.A. Mayer, Chem. Rev. 102 (2002) 3543.

[4] E. Torres, I. Bustos-Jaimes, S. Le Borgne, Appl. Catal. B 46 (2003) 1.

[5] K.M. Choi, S. Ikeda, S. Ishino, K. Ikeue, M. Matsumura, B. Ohtani, Appl. Catal. A 278 (2005) 269.

[6] K.S. Suslick, in: K.M. Kadish, K.M. Smith, R. Guilard (Eds.), The Porphyrin Handbook, vol.4, Academic Press, New York, 2000, pp. 41-63.

[7] D. Lee, J. Mol. Catal. A 151 (2000) 71.

[8] R.A. Reziq, D. Avnir, I. Miloslavski, H. Schumann, J. Blum, J. Mol. Catal. 185 (2002) 179. 
[9] A.P. Wight, M.E. Davis, Chem. Rev. 102 (2002) 3589.

[10] Z.L. Lu, E. Lindner, H. Mayer, Chem. Rev. 102 (2002) 3543.

[11] J.M. Thomas, R. Raja, D.W. Lewis, Angew. Chem. Int. Ed. 44 (2005) 6456.

[12] R.M. Gauvin, L. Delevoye, R.A. Hassan, J. Keldenich, A. Mortreux, Inorg. Chem. 46 (2007) 1062.

[13] J.T. Lutz, Jr., in: Kirk-Othmer, M. Grayson, D. Eckroth, G.J. Bushey, C.I. Eastman, A. Klingsberg, L. Spiro (Eds.), 3rd ed., Encyclopedia of Chemical Technology, vol. 9, Wiley, New York, 1980, pp 251-266.

[14] G. Sienel, R. Rieth, K.T. Rowbottom, in: Ullmann's Encyclopedia of Industrial Chemistry (on-line version), Epoxides, John Wiley \& Sons, 2008.

[15] L. Canali, D.C. Sherrington, Chem. Soc. Rev. 28 (1999) 85.

[16] D.E. De Vos, B.F. Sels, P.A. Jacobs, Adv. Synth. Catal. 345 (2003) 457.

[17] M.T. Musser, in: Ullmann's Encyclopedia of Industrial Chemistry (on-line version), Adipic Acid, John Wiley \& Sons, 2008.

[18] J.D. Koola, J.K. Kochi, J. Org. Chem. 52 (1987) 4545.

[19] J.T. Groves, J. Inorg. Biochem. 100 (2006) 434.

[20] J.-F. Lambert, G. Poncelet, Topics Catal. 4 (1997) 43.

[21] P. Cool, E. Vansant, in: H.G. Karge, J Weitkamp, (Eds.), Molecular Sieves Science and Technology, Volume 1, Synthesis, Springer, Berlin and Heidelberg, 1998, pp. 265-288.

[22] A. Gil., L.M. Gandía, M.A. Vicente, Catal. Rev. 42 (2000) 145.

[23] Z. Ding, T.J. Kloprogge, R.L. Frost, G.Q. Lu, H.Y. Zhu, J. Porous Mater. 8 (2001) 273.

[24] F. Bergaya, A. Aouad, T. Mandalia, in: Handbook of Clay Science, F. Bergaya, B.K.G. Theng, G. Lagaly (Eds.), Elsevier, Amsterdam, 2004, pp 393-422.

[25] E.M. Serwicka, K. Bahranowski, Catal. Today, 90 (2004) 85.

[26] J.T. Kloprogge, L.V. Duong, R.L. Frost, Environ. Geol. 47 (2005) 967.

[27] A. Gil, S.A. Korili, M.A. Vicente, Catal. Rev. 50 (2008) 153.

[28] W. Nam, S.H. Kim, S.J. Kim, R.Y.N. Ho, J.S. Valentine, Inorg. Chem. 35 (1996) 1045.

[29] F. Zaera, Catal. Today 81 (2003) 149.

[30] R. Toranzo, M.A. Vicente, M.A. Bañares-Muñoz, Chem. Mater. 9 (1997) 1829.

[31] N. Lahav, U. Shani, J. Shabtai, Clays Clay Miner. 26 (1978) 107.

[32] J.Y. Bottero, J.M. Cases, F. Fiessinger, J.E. Poirier, J. Phys. Chem. 84 (1980) 2933. 
[33] S.M. Bradley, R.A. Kydd, R.J. Yamdagni, Chem. Soc. Dalton Trans. (1990) 2653.

[34] M. Che, O. Clause, Ch. Marcilly, in: G. Ertl, H. Knözinger, J. Weitkamp (Eds.), Preparation of Solid Catalysts, Wiley-VCH, Weiheim, 1999, pp. 315-340.

[35] F. Rouquerol, J. Rouquerol, K. Sing, Adsorption by powders and porous solids. Principles, Methodology and Applications, Academic Press, London, 1999.

[36] J.G. Sharefkin, H. Saltzmann, Org. Synth. 43 (1963) 62.

[37] H.J. Lucas, E.R. Kennedy, M.W. Forno, Org. Synth. 43 (1963) 483.

[38] C. Belver, G. Mata, R. Trujillano, M.A. Vicente, Catal. Lett. 123 (2008) 32.

[39] JCPDS File, Joint Committee on Powder Diffraction Standards, International Centre for Diffraction Data, Philadelphia, 1977.

[40] K. Utchariyajit, E. Gulari, S. Wongkasemjit, Appl. Organomet. Chem. 20 (2005) 81.

[41] S.J. Zhang, L.D. Li, B. Xue, J.X. Chen, N.J. Guan, F.X. Zhang, Reac. Kinet. Catal. Lett. 89 (2006) 81.

[42] M. Jitianu, A. Jitianu, M. Zaharescu, D. Crisan, R. Marchitan, Vib. Spectrosc. 22 (2000) 75.

[43] T. Kawabata, Y. Shinozuka, Y. Ohishi, T. Shishido, K. Takaki, K. Takehira, J. Mol. Catal. A 236 (2005) 206.

[44] L. Palombi, F. Bonadies, A. Scettri, J. Mol. Catal. A 140 (1999) 47.

[45] J.S. Daniels, K.S. Gates, J. Am. Chem. Soc. 118 (1996) 3380.

[46] T. Punniyamurthy, M.M. Reddy, S.J.S. Kalra, J. Iqbal, Pure Appl. Chem. 68 (1996) 619.

[47] R. Ferreira, H. García, B. de Castro, C. Freire, Eur. J. Inorg. Chem. (2005) 4272.

[48] J.Y. Wang, G.G. Xia, Y.G. Yin, S.L. Suib, C.L. O'Young, J. Catal. 176 (1998) 275.

[49] J.M. Thomas, R. Raja, G. Sankar, R.G. Bell, Acc. Chem. Res. 34 (2001) 191.

[50] P. Inchley, J.R. Lindsay-Smith, R.J. Lower, New. J. Chem. 13 (1989) 669.

[51] T. Nagataki, K. Ishii, Y. Tachi, S. Itoh, Dalton Trans. (2007) 1120.

[52] M. Costas, K. Chen, L. Que Jr., Coord. Chem. Rev. 200-202 (2000) 517.

[53] H.B. Dunford, Coord. Chem. Rev. 233-234 (2002) 311.

[54] R. Raja, G. Sankar, J.M. Thomas, J. Am. Chem. Soc. 121 (1999) 11926.

[55] P. Oliveira, M.L. Rojas-Cervantes, A.M. Ramos, I.M. Fonseca, A.M. Botelho do Rego, J. Vital. Catal. Today 118 (2006) 307. 



\section{Capítulo VI I}

Preparation and characterization of new Ni-aluminosilicate catalysts and their performance in the epoxidation of (Z)-cyclooctene 



\section{Abstract}

This work reports the preparation of two series of Ni-aluminosilicates by the solgel and the co-precipitation methods. The main difference between the two processes of synthesis is the $\mathrm{pH}$ of the initial mixture, 2 for the sol-gel and 11 for the co-precipitation method, respectively. The obtained solids are heat-treated at various temperatures in the range of room temperature to $1000{ }^{\circ} \mathrm{C}$, and the resulting materials are characterized by X-ray diffraction, thermogravimetric and differential thermal analysis, ultraviolet-visible spectroscopy, nitrogen adsorption, and transmission electron microscopy. Various nickel compounds crystallize under the sol-gel reaction conditions, as well as, amorphous silica and crystalline $2 \mathrm{NaAlO}_{2} \cdot 3 \mathrm{H}_{2} \mathrm{O}$. Ni-Al hydrotalcite, takovite, and amorphous silica are obtained when the samples from the co-precipitation method are analysed. A maximum of $302 \mathrm{~m}^{2} / \mathrm{g}$ of specific surface area shows the co-precipitation samples series, with only $32 \mathrm{~m}^{2} / \mathrm{g}$ in the case of the sol-gel samples series. The catalytic performance in the epoxidation of $(Z)$-cyclooctene at room temperature and atmospheric pressure is evaluated using aqueous hydrogen peroxide as oxidant.

Keywords: nickel aluminosilicate, saponite, sol-gel synthesis, co-precipitation synthesis, epoxidation

\section{I.1. I ntroduction}

Among the various clay materials, saponite is characterized by a high negative charge, generated by a high degree of Si-Al substitution in the tetrahedral sheet, and by a high occupancy of the octahedral layer [1]. $\mathrm{Mg}$ is the most usual divalent cation, although saponites with high content of $\mathrm{Fe}^{2+}$ have also been described [2]. The high occupancy in the octahedral layer, together with a certain degree of substitution by trivalent cations, makes the negative charge in this sheet to be very low, sometimes close to zero, and even slowly positive. Thus, the negative charge of the clay is concentrated in the tetrahedral sheet, which differentiates saponite from the most known and used smectite material, montmorillonite, in which the charge is mainly located on the octahedral sheet. 
The amount and location of the clay charge gives saponite a particular interest from the catalytic point of view, because of the direct relation between it and the acidity of the clay [3]. In the preparation of pillared solids, the charge has a great importance regulating the facility for intercalation, the cross-linking between the clay layers and the pillars, and the thermal stability, remarkably higher than for montmorillonites [3].

The incorporation of transition elements to the structure of clays has a high interest, and may allow combining the high surface area and porosity of the clays with the catalytic properties of such elements. This incorporation has sometimes been done by means of impregnation treatments, although in this case the impregnating elements are located only on the surface of the clay, or may form new phases by dissolution of small amounts of the clays in the impregnation treatments. An alternative strategy is the synthesis of clays containing these cations in the octahedral positions. Also in this sense, saponite is an excellent candidate, it may be noticed that the theoretical formula of a saponite is, in the basis of 22 oxygen atoms, as follows: $\mathrm{M}_{x}\left[\mathrm{Si}_{8-x} \mathrm{Al}_{\mathrm{x}}\right]\left[\mathrm{M}_{3}^{\prime}\right] \mathrm{O}_{20}(\mathrm{OH})_{4} \cdot \mathrm{nH}_{2} \mathrm{O}$. This theoretical formula considers that the octahedral layer of the clay is completely occupied by the divalent $\mathrm{M}^{\prime}$ metal, and all the charge of the clay is due to isomorphous substitution of $\mathrm{Si}(\mathrm{IV})$ by $\mathrm{Al}(\mathrm{III})$ in the tetrahedral layer, the negative charge thus created being compensated by the exchangeable cations, expressed in the formula as monovalent $M$ cation. A large series of synthetic saponites with variable charge, with $x$ varying between 0.8 and 1.5 in the cell formula, having the octahedral layer completely occupied by $\mathrm{Mg}$, and $\mathrm{Na}$ being the chargecompensating cation, have been synthesized by Robert et al. [4], and used for pillaring experiments $[4,5]$. These syntheses were carried out under hydrothermal conditions, at $400^{\circ} \mathrm{C}$ and 1000 bar, and run of four weeks, conditions not easy to reach for the synthesis of large amount of solids.

Epoxides can be converted into alcohols, polyethers and aldehydes, compounds that show widespread applications in chemical and pharmaceutical industry [6]. The use of several oxidants, as oxygen, hydrogen peroxide, tert-butylhydroperoxide, etc., has been reported in the literature for the production of epoxides from alkenes [7]. Hydrogen peroxide gives a clean and environmental friendly reaction since the starting material is safe and inexpensive and only water is formed as a by-product. Reactions with hydrogen peroxide require generally a catalyst and, in this way, 170 
several efficient catalytic systems for epoxidation with hydrogen peroxide have been developed $[8,9,10]$.

In this work, we try to prepare in an easy way synthetic saponites containing $\mathrm{Ni}^{2+}$ in the octahedral layer. For that, reaction between $\mathrm{Si}, \mathrm{Al}, \mathrm{Ni}$ and $\mathrm{Na}$ reagents have been checked by means of sol-gel and co-precipitation methods. Silicate layers are obtained only by the first method, despite they do not adopt a large ordering in the c-axis. The solids obtained can be viewed as nanocomposites of $\mathrm{Ni}$ phases over aluminosilicate supports and they have been used as catalysts in the epoxidation of $(Z)$-cyclooctene.

\section{VII.2. Experimental}

\section{VII.2.1. Preparation of the solids}

The synthesis of the solids was carried out by two methods, namely sol-gel and co-precipitation, following the procedures we have reported for the synthesis of other families of solids $[10,11]$.

2.1.1. Sol-gel. The process begins by dissolving $4.37 \mathrm{~g}$ of $\mathrm{NiCl}_{2} \cdot 6 \mathrm{H}_{2} \mathrm{O}$ (Merck, $97 \%$ ), $0.33 \mathrm{~g}$ of $\mathrm{Na}_{2} \mathrm{CO}_{3}$ (Merck, 99\%) and $2.04 \mathrm{~g}$ of anhydrous $\mathrm{AlCl}_{3}$ (Merck, $99.999 \%)$ in $60.0 \mathrm{~cm}^{3}$ of dichloromethane, in a two-neck flask. Immediately, $4.75 \mathrm{~cm}^{3}$ of tetraethylorthosilicate (TEOS, Sigma-Aldrich, 99.999) were added, and then $10.0 \mathrm{~cm}^{3}$ of isopropyl ether anhydrous (Aldrich, 99.0). The $\mathrm{pH}$ of the mixture was 2.0. The mixture was maintained under reflux conditions, at $110{ }^{\circ} \mathrm{C}$ and under argon atmosphere, with magnetic stirring for 4 hours. After reflux, the mixture was cooled and aged overnight, at room temperature. The solvent was then removed under vacuum, using a rotaevaporator. The solid was washed with several solvents in the following order: dichloromethane, acetonitrile, and methanol. Then, the solid was dried in an oven, at $70{ }^{\circ} \mathrm{C}$ for 48 hours. The resulting solid was named SGdried (SG denoting sol-gel procedure) and it was submitted to thermal treatments at $300,450,600$ and $1000{ }^{\circ} \mathrm{C}$, heating it at a rate of $30^{\circ} \mathrm{C} / \mathrm{min}$, and maintaining it for 4 hours at the calcination temperatures. The resulting solids were named SG-300, SG-450, SG-600 and SG-1000, respectively. For comparative purposes, a synthesis was carried out in absence of nickel, maintaining all the other variables of the process, the dried solid thus obtained was denoted as SG-blank (it was not calcined). 
2.1.2. Co-precipitation. In this case, the process begins by dissolving $4.38 \mathrm{~g}$ of $\mathrm{NiCl}_{2} \cdot 6 \mathrm{H}_{2} \mathrm{O}, 0.16 \mathrm{~g}$ of $\mathrm{Na}_{2} \mathrm{CO}_{3}$ and $2.00 \mathrm{~g}$ of anhydrous $\mathrm{AlCl}_{3}$ in $60.0 \mathrm{~cm}^{3}$ of dichloromethane anhydrous (Aldrich, 99.8\%) in a two-neck flask. Immediately, $6.00 \mathrm{~cm}^{3}$ of concentrated ammonia (Fluka, 97\%) were added, followed by the addition of $4.75 \mathrm{~cm}^{3}$ of TEOS. At this moment, a gelification of the mixture was observed, being added $40.0 \mathrm{~cm}^{3}$ of water and $9.0 \mathrm{~cm}^{3}$ of ammonia. The $\mathrm{pH}$ of the mixture was 11.0 . The mixture was maintained under reflux conditions, at $60^{\circ} \mathrm{C}$ and with magnetic stirring, for 20 hours. The resulting solid was named CP-dried ( $\mathrm{CP}$ denoting co-precipitation procedure), and it was submitted to washing, drying and calcination processes similar to those described in the former method. The solids obtained were denoted CP-300, CP-450, CP-600 and CP-1000, respectively. As in the previous method, a comparative synthesis in absence of nickel was carried out, the dried solid thus obtained was denoted as CP-blank.

\section{VII.2.2. Characterization techniques}

Chemical analysis for $\mathrm{Si}, \mathrm{Al}, \mathrm{Ni}$ and $\mathrm{Na}$ were carried out using atomic absorption in a Mark-II ELL-240 instrument in Servicio General de Análisis Químico Aplicado (University of Salamanca, Spain).

X-ray diffraction (XRD) patterns of the solids were obtained over non-oriented powder samples, between 2 and $70^{\circ}$ of $2 \theta$ with a scanning velocity of $2 \% / \mathrm{min}$, by using a Siemens D-500 diffractometer, at $40 \mathrm{kV}$ and $30 \mathrm{~mA}$, and employing $\mathrm{Cu} \mathrm{K} \alpha$ filtered radiation.

Thermogravimetric analysis (TGA) and differential thermal analysis (DTA) were carried out using a Thermal Analyst TA Instrument SDT Q 600 Simultaneous DTATGA-DSC, in nitrogen (Air Liquide, $99.999 \%, 35 \mathrm{~cm}^{3} / \mathrm{min}$ ), at a heating rate of $20^{\circ} \mathrm{C} / \mathrm{min}$, from $25^{\circ} \mathrm{C}$ to $1500^{\circ} \mathrm{C}$.

Electronic spectra were obtained in a Hewlett-Packard 8453, Diode Array UV-Vis spectrophotometer. The spectra of the solids in dichloromethane were recorded in a 2.0-mm path length cell. Dichloromethane was the solvent of choice because it led to improved UV-Vis spectra when the suspension was prepared. The spectra were preprocessed by using wavelets filter 44.45 with the Dubauchie (db4) base function for noise minimization, subtraction of the isotonic medium spectrum, multiplicative 
scattering correction 46 (MSC) to minimize Raleigh scattering due to variation in the refraction index for the matrix, and a second degree polynomial fit to minimize matrix fluorescence.

Textural analyses were carried out from the corresponding nitrogen (Air Liquide, $99.999 \%)$ adsorption at $-196{ }^{\circ} \mathrm{C}$, obtained from a static volumetric apparatus (Micromeritics ASAP 2010 adsorption analyser). The samples, $0.2 \mathrm{~g}$, were degassed at $200{ }^{\circ} \mathrm{C}$ for $24 \mathrm{~h}(\mathrm{p}<0.133 \mathrm{~Pa})$. Specific surface area was obtained from the BET method, the external surface area by means of the $t$ method, and the total pore volume was estimated from the amount of nitrogen adsorbed at a relative pressure of 0.95 .

Scanning electron microscopy (SEM) of the materials was performed on ground samples. The samples were coated with a conducting layer of gold by sputter coating. The images were obtained in a Digital Scanning Microscope DSM 960 Zeiss.

\section{VII.2.3. Catalytic performance}

The epoxidation of (Z)-cyclooctene with hydrogen peroxide was used to evaluate the catalytic behaviour of the solids. Hydrogen peroxide, $70 \mathrm{wt}$. \%, used as received was added to a $4 \mathrm{~cm}^{3}$ vial sealed with a teflon-coated silicone septum containing one of the catalysts $(10 \mathrm{mg})$, in a mixture $1: 1$ of the solvents $1,2-$ dichloroethane : acetonitrile $\left(1 \mathrm{~cm}^{3}\right) ;(Z)$-cyclooctene (previously purified on alumina column) as substrate $(1.15 \mathrm{mmol})$; and cyclohexanone as internal standard $\left(5 \mathrm{~cm}^{3}\right)$. After 24 hours, the reaction products were analysed by gas chromatography (HP 6890). Yields were determined by comparison with pure compounds by using calibration curves, and as indicated before using cyclohexanone as internal standard. A control of the reactions was carried out in the absence of the nickel ions, by using the blank solids above described, blank tests.

\section{VII.3. Results and discussion}

\section{VII.3.1. Chemical analysis}

The chemical composition of the synthesized solids is given in Table 1. Although the as-synthesized non-calcined solids were actually analysed, the composition is given in wt. \% of oxides, referred to water-free solids, that is, the sum of the 
corresponding oxides normalised to $100 \%$. As the solids in a given series only differ in the calcination treatment, their chemical water-free composition is obviously the same. For each series, the composition of the blank solid is also offered.

\begin{tabular}{ccccc}
\hline Sample & $\mathrm{SiO}_{2}$ & $\mathrm{Al}_{2} \mathrm{O}_{3}$ & $\mathrm{NiO}$ & $\mathrm{Na}_{2} \mathrm{O}$ \\
\hline SG-blank & 56.0 & 34.1 & --- & 9.9 \\
SG & 24.3 & 25.4 & 44.3 & 6.0 \\
CP-blank & 61.6 & 36.9 & --- & 1.5 \\
CP & 35.6 & 26.6 & 37.9 & n.d.
\end{tabular}

Table 1. Chemical analyses of the solids from each series (wt. \%).

${ }^{a}$ Results referenced to ignited solids ( $0 \%$ water). n.d. $=$ not detected.

The solids in the SG series contain large amounts of the four reacting elements. The very high amount of nickel is especially remarkable. The composition of the solid is easily comprehensible considering the compounds present in it (vide infra), various $\mathrm{Ni}$ compounds crystallize under the reaction conditions. Besides, a certain amount of all the elements are forming silicate layers. Si is mainly forming amorphous silica, Al forming crystalline $2 \mathrm{NaAlO}_{2} \cdot 3 \mathrm{H}_{2} \mathrm{O}$ and probably also an amorphous phase, and $\mathrm{Na}$ also taking part of $2 \mathrm{NaAlO}_{2} \cdot 3 \mathrm{H}_{2} \mathrm{O}$ (see Figure 1 ).

The chemical composition of the $\mathrm{CP}$ solid is rather different. The only phases detected in the solid by XRD (see Figure 2) were Ni-Al hydrotalcite, takovite, and amorphous silica. The first important difference of the chemical composition of this solid with respect to that of the SG series is the total absence of $\mathrm{Na}$. This is logical considering that this element does not take part of the composition of the hydrotalcite neither of the silica, although it may be expectable some amount of it be retained by the precipitated solid. It is remarkable that the atomic ratio between $\mathrm{Ni}: \mathrm{Al}$ is very close to $1: 1$, while the chemical composition of takovite shows a ratio $3: 1$. That means that all $\mathrm{Ni}$ is in the sample as takovite, while only about $1 / 3$ of $\mathrm{Al}$ forms this compound, the other $2 / 3$ may be forming an amorphous phase.

In absence of $\mathrm{Ni}$, the blank solids of each series are composed by large amounts of $\mathrm{Si}$ and $\mathrm{Al}$, and it is remarkable that also for these blank solids the amount of $\mathrm{Na}$ is much lower in the solid obtained by co-precipitation that under sol-gel conditions. This suggests that the incorporation of $\mathrm{Na}$ to the silico-alumina phases precipitated 
is easier under sol-gel conditions, which partially justifies, together with the existence of $\mathrm{Na}$-containing phases, the high amounts of $\mathrm{Na}$ in SG-series solids.

\section{VII.3.2. X-ray diffraction}

The nature of the solids obtained by the two synthesis procedures is also different. The XRD patterns of the solids obtained by the sol-gel procedure are shown in Figure 1. As indicated in the Experimental section, we first carried out a synthesis in the absence of nickel. The XRD pattern of this solid shows the formation of a layered compound, with a basal spacing of $12.86 \AA$ and which matches with the JCPDS pattern numbers 14-0298, 38-0237 and 37-0241, all of them corresponding to "Sodium Aluminum Silicate Hydrates" [12]. However, intense crystalline peaks corresponding to the reagents, mainly of $\mathrm{NaCl}$ (pattern number 5-0628, halite syn) are also observed in the diffractogram, as also a wide band between $20-30^{\circ}$ of 2 theta, characteristic of silica (the amorphous halo). So, although a certain amount of a silicate phase is formed, an important part of the reagents does not react.

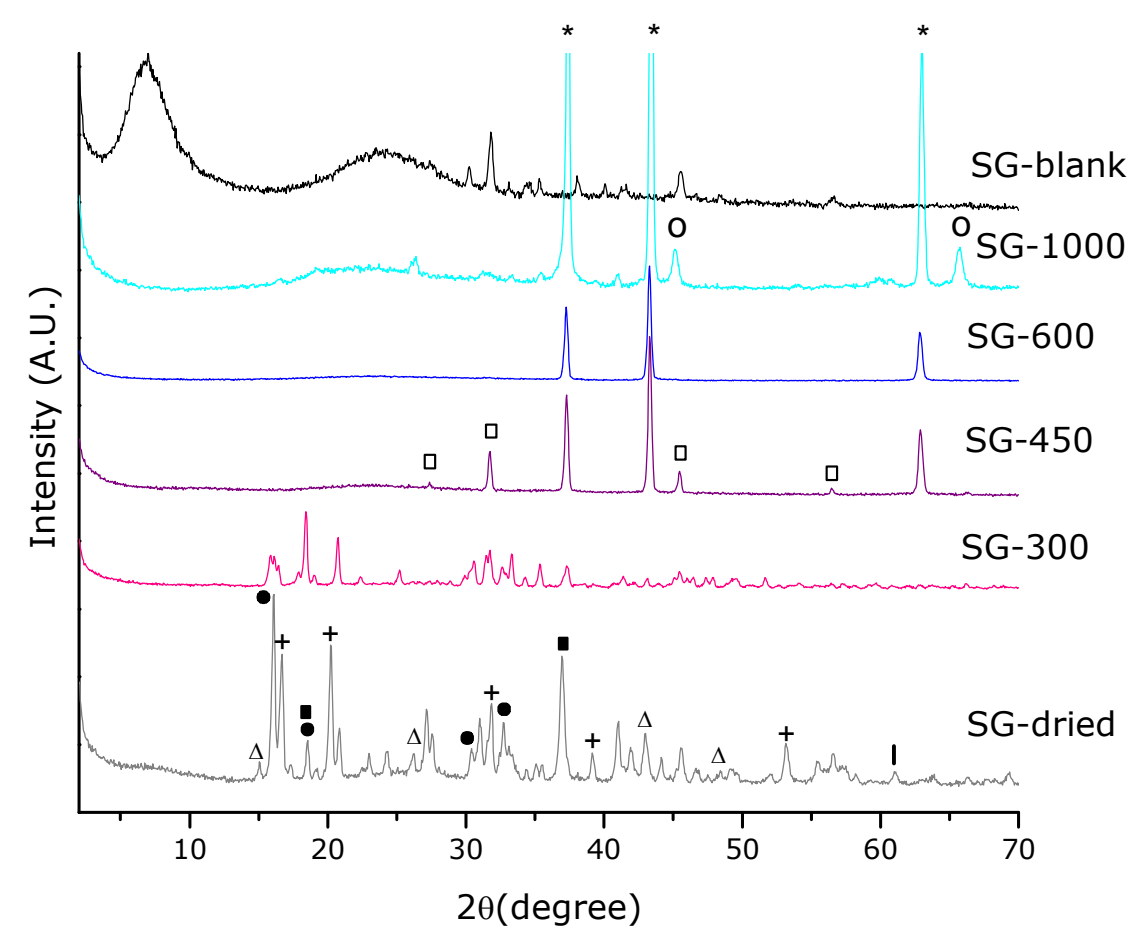

Figure 1. X-ray powder diffraction patterns of the SG series solids $\left(*=\mathrm{NiO} ; \mathrm{O}=\mathrm{NiAl}_{2} \mathrm{O}_{4}\right.$;

$\square=\mathrm{NaCl} ; \Delta=\mathrm{Ni}\left(\mathrm{HCO}_{3}\right)_{2} ; \bullet=\mathrm{NiCl}_{2} \cdot 6 \mathrm{H}_{2} \mathrm{O} ; \boldsymbol{\|}=\mathrm{Ni}_{3} \mathrm{O}_{2}(\mathrm{OH})_{4} ;+=2 \mathrm{NaAlO}_{2} \cdot 3 \mathrm{H}_{2} \mathrm{O} ;$

I = phyllosilicate). 
The diffractogram is completely different for the sample SG-dried, prepared under the same conditions but in the presence of nickel. Before discussing this diffractogram and considering that the first objective of this work was the preparation of synthetic saponites, and having in mind the theoretical formula of this clay above indicated, the saponite which synthesis we planned was: $\mathrm{Na}_{1}\left[\mathrm{Si}_{7} \mathrm{Al}_{1}\right]\left[\mathrm{Ni}_{3}\right] \mathrm{O}_{20}(\mathrm{OH})_{4} \cdot \mathrm{nH}_{2} \mathrm{O}$. For that, the ratio between the different elements in the reaction medium was $\mathrm{Si}: \mathrm{Al}: \mathrm{Ni}: \mathrm{Na}$ of $7: 5: 6: 2$. Thus, assuming that if clay layers are formed the total occupation of the octahedral sheet by $\mathrm{Ni}^{2+}$ may be difficult, and as $\mathrm{Al}^{3+}$ is also able to occupy this sheet, an amount of $\mathrm{Al}$ higher than that necessary for substituting $\mathrm{Si}$ in the tetrahedral layer was added. The excess of Al can, at the same time, increase the extent of the isomorphous tetrahedral substitution $(x>1)$, considering that up to a substitution degree of 1.5 of each 8 positions has been reported [4,5], which would increase the total charge of the clay, reason by which an excess of $\mathrm{Na}$ was also added.

The diffractogram of SG-dried sample is complex, but it can be easily decomposed in various compounds. The first one is $\mathrm{NiCl}_{2} \cdot 6 \mathrm{H}_{2} \mathrm{O}$, nickelbischofite syn, pattern number 25-1044, which is the only reagent which peaks are in the synthesized solid. Besides, two other $\mathrm{Ni}$-compounds are found, namely $\mathrm{Ni}\left(\mathrm{HCO}_{3}\right)_{2}$, pattern number 15-0782, and $\mathrm{Ni}_{3} \mathrm{O}_{2}(\mathrm{OH})_{4}$, pattern number 6-0144, and also a NaAl compound, namely $2 \mathrm{NaAlO}_{2} \cdot 3 \mathrm{H}_{2} \mathrm{O}$, pattern number $2-1025$. This suggests that a certain amount of each element tends to crystallize in simple phases, implying one or two metals, silicium may be forming amorphous phases not detected by the high crystallinity of the mentioned compounds. However, once considered the effects from these compounds, the characteristic pattern of a layered silicate can be observed. This is detected by the presence of a low intense but well-defined band centred at $12.89 \AA$ and by the presence of a small peak at $1.52 \AA$. This last effect is characteristic of the clay layers, corresponding to the reflection effect denoted 06,33 , which is actually the sum of 060 and 330 effects, both being independent of the c-axis stacking. In other words, this effect is present in a solid composed of individual layers, even if they are not ordered in the c dimension. This effect strongly depends on the size of the octahedral cations, varying in natural clays between $1.49 \AA$ when these positions are majority occupied by $\mathrm{Al}^{3+}$ and $1.53 \AA$ when these positions are majority occupied by $\mathrm{Mg}^{2+}[1]$. The position of this peak in our solid, intermediate between these both values, agrees with the trend observed in the ionic ratio of these cations in octahedral coordination, that of $\mathrm{Ni}^{2+}(0.69 \AA)$ 
being also intermediate between those of $\mathrm{Mg}^{2+}(0.72 \AA)$ and $\mathrm{Al}^{3+}(0.535 \AA)$ [13]. It may also be indicated that although this peak is centred at $1.52 \AA$, it is asymmetric, even after subtracting $\mathrm{k \alpha}_{2}$ radiation, with a shoulder at higher values of angle, that is, at lower values of basal spacing, which may correspond to the occupancy of part of the octahedral positions by $\mathrm{Al}^{3+}$ cations. Thus, the formation of individual layers with both $\mathrm{Ni}^{2+}$ and $\mathrm{Al}^{3+}$ in the octahedral sites may be proposed. The stacking of these layers along the c-axis does not reach a high crystallinity, but it is not negligible, as indicated previously an effect centred at $12.89 \AA$ is clearly observed. This is the basal spacing reported for saponites with a monolayer of water molecules in the interlayer region. It may be considered that the minimum basal spacing of this clay, TOT separation, that is, only the layers stacked without any molecule in the interlayer region, is $9.6 \AA$, and when the clay is hydrated with a bilayer of water molecules, the basal spacing increases to about $14 \AA$ [14]. So, the formation of saponite layers, with a partial stacking on the c-axis and a monolayer of water in the interlayer region may be proposed.

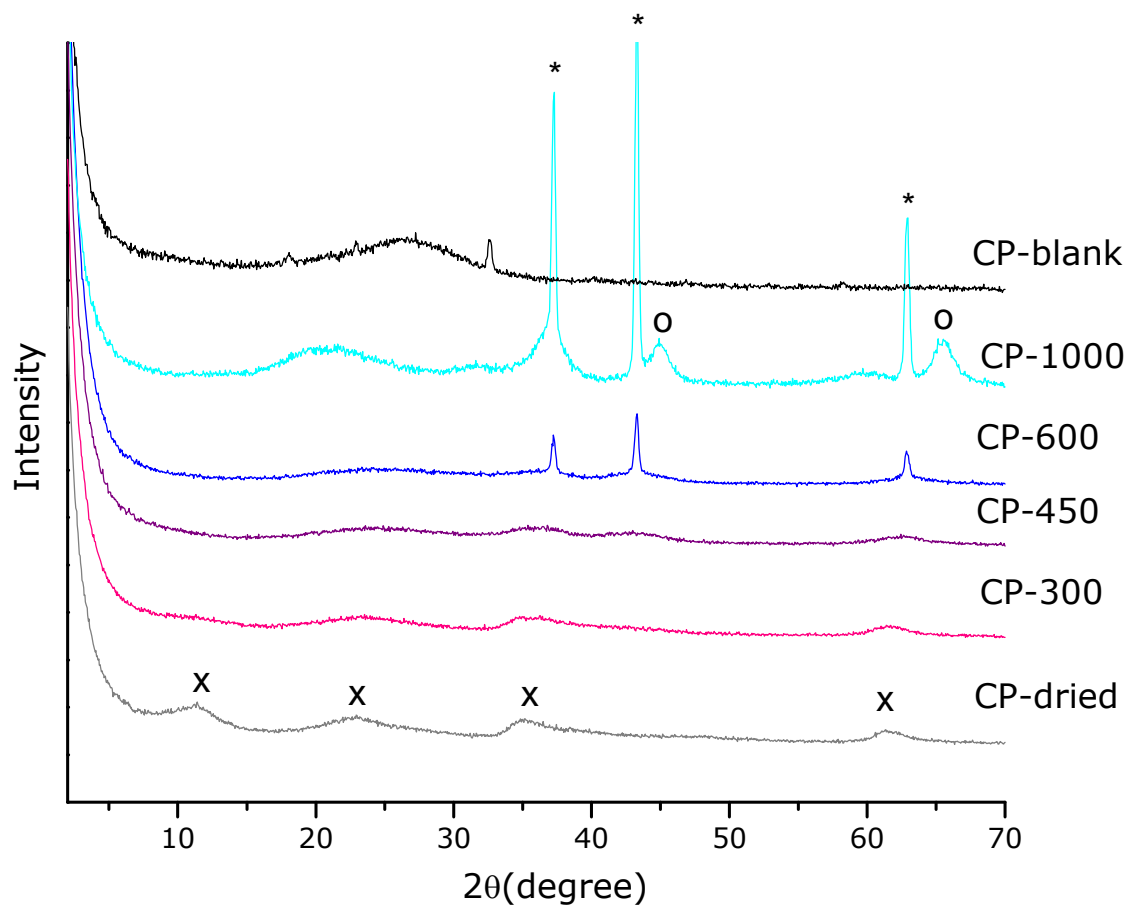

Figure 2. X-ray powder diffraction patterns of the $\mathrm{CP}$ series solids $\left(*=\mathrm{NiO} ; 0=\mathrm{NiAl}_{2} \mathrm{O}_{4} ; \mathrm{x}=\right.$ takovite $)$.

The behaviour of the synthesized solid under calcination at increasing temperature can be also observed in Figure 1 . Calcination at $300{ }^{\circ} \mathrm{C}$ mainly 
produces the modification of the hydrated phases before described, being particularly interesting the collapse of the silicate layers before stacked, observed by the disappearance of the effect at $12.89 \AA$. More interesting is the calcination at $450{ }^{\circ} \mathrm{C}$, which produces the apparition of very crystalline effects from $\mathrm{NiO}$ (pattern number 4-0835, bunsenite syn) and $\mathrm{NaCl}$, and also of the halo of amorphous silica, while Al may be present as not-detectable amorphous phases. The effects corresponding to $\mathrm{NaCl}$ disappear when calcining at $600^{\circ} \mathrm{C}$, the diffractogram of the solid obtained after heating at this temperature showing the effects of crystalline $\mathrm{NiO}$ over amorphous silica, although it may be noticed again that $\mathrm{Al}$ and $\mathrm{Na}$ remain in the solid, forming amorphous phases, such as Na-aluminate. Calcination at $1000{ }^{\circ} \mathrm{C}$ produces the beginning of the crystallization of $\mathrm{NiAl}_{2} \mathrm{O}_{4}$ spinel, pattern number 10-0339, which effects are observed together with those of $\mathrm{NiO}$ and of the amorphous wide band of silica. Formation of mullite was not detected.

The synthesis goes in a very different way when the co-precipitation method is used. The diffractograms of the solids from this series are shown in Figure 2. First, the diffractogram of the blank synthesis, without nickel, is shown, the characteristic amorphous halo of silica and small peaks from $\mathrm{NaCl}$ being the only effects observable. The dried solid obtained in presence of nickel show a pattern characteristic of a layered compound, with a basal spacing of $7.76 \AA$, and all the effects observed in the diffractogram matching with those from Ni-Al hydrotalcite, takovite, pattern number 15-0087. Hydrotalcite, a natural mineral with formula $\left[\mathrm{Mg}_{6} \mathrm{Al}_{2}(\mathrm{OH})_{16}\right] \mathrm{CO}_{3} \cdot 4 \mathrm{H}_{2} \mathrm{O}$, gives the name to a large group of layered double hydroxides (LDHs), also named anionic clays. Their structure can be easily described as sheets of $\left[\mathrm{M}^{\mathrm{II}}(\mathrm{OH})_{6}\right]$ octahedra sharing edges, brucite structure, with a partial $\mathrm{M}^{\mathrm{II}} / \mathrm{M}^{\mathrm{III}}$ substitution in these layers. This substitution produces a positive charge, compensated by interlayer anions. Water molecules also exist in the interlayer region. The nature of both the divalent and trivalent layer cations can be easily changed, and thus a lot of synthetic hydrotalcites have been prepared $[15,16]$. If $\mathrm{Mg}^{2+}$ is completely replaced by $\mathrm{Ni}^{2+}$, and $\mathrm{Al}^{3+}$ continues being the only trivalent cation, the resulting hydrotalcite is named takovite, $\left[\mathrm{Ni}_{6} \mathrm{Al}_{2}(\mathrm{OH})_{16}\right] \mathrm{CO}_{3} \cdot 4 \mathrm{H}_{2} \mathrm{O}[17]$.

So, under these synthesis conditions, $\mathrm{Ni}$ and $\mathrm{Al}$ reacts giving rise to the corresponding hydrotalcite-like compound, takovite, although with our synthesis conditions it may contain chloride, carbonate or both as interlayer charge- 
compensating cations. It may be remarked that the substitution carbonate-chloride as interlayer anions in hydrotalcite-like compounds does not produce any significant change in their basal spacings. Si and $\mathrm{Na}$ do not form any crystalline compound, although the amorphous band of silica is detected in the diffractogram. It is remarkable that the takovite obtained under our synthesis conditions is poorly crystalline, as shown by the low intensity of the diffraction peaks and also by the fact that the effect at about $62^{\circ}$ of $2 \theta$ appears as a wide band, when it appears as two well-differentiated peaks in well-crystallized samples. This is confirmed by the thermal evolution of this solid when it is heated at 300 or $450{ }^{\circ} \mathrm{C}$, it is observed that although the rest of the peaks of the hydrotalcite structure are maintained, the first one disappears, showing the collapse of the layers that were ordered in the dried sample.

The solid calcined at $600{ }^{\circ} \mathrm{C}$ show crystalline peaks from NiO, and also a low intense amorphous band. This suggests that $\mathrm{Ni}$ is segregated from the hydrotalcite structure forming a simple oxide, while Al may give an amorphous phase. However, $\mathrm{Ni}$ and $\mathrm{Al}$ react again at higher temperature, the diffractogram of the solid obtained after heating at $1000{ }^{\circ} \mathrm{C}$ shows, together with the peaks of $\mathrm{NiO}$, those of $\mathrm{NiAl}_{2} \mathrm{O}_{4}$ spinel, although it is remarkable that the spinel phase is much less crystalline than in the solid obtained at the same temperature from the sol-gel method. Besides, the band corresponding to amorphous silica is also present. So, these last solids can be viewed as $\mathrm{NiO}$ particles when calcined at $600{ }^{\circ} \mathrm{C}$, and $\mathrm{NiO}$ and $\mathrm{NiAl}_{2} \mathrm{O}_{4}$ particles when calcined at $1000^{\circ} \mathrm{C}$, over a silicoalumina support, Na probably being in the amorphous phase. As in the previous method, mullite is not detected.

\section{VII.3.3. Thermal analyses}

The thermal analyses of the SG-dried sample are shown in Figure 3. The sample underwent a strong weight loss, c.a. 7 wt. \%, with an associated endothermic transition centred at $150{ }^{\circ} \mathrm{C}$, corresponding to the loss of coordination or physisorbed water molecules, weakly bound to the materials. This weight loss is actually the sum of three processes, as observed in the DTG curve, and also in the DTA one, which show a shoulder with two components at higher temperatures. This is in agreement with the fact that the solid is composed of several phases, in which water may be retained with several intensities. A very low weight loss, with a very weak endothermal associated effect is observed at c.a. $590{ }^{\circ} \mathrm{C}$, assigned to the 
removal of carbonate groups. A clear weight loss (c.a. 2\%) is located between 700$800{ }^{\circ} \mathrm{C}$, also observed as an endothermal effect, and which corresponds to the elimination of the hydroxyl groups, thus transforming the solid to a mixture of oxide phases. This is in agreement with the transformations above described from $\mathrm{XRD}$ results, although $\mathrm{NiO}$ is formed at lower temperature, the formation of spinel does not begin up to the loss of hydroxyl groups in the alumina phase.

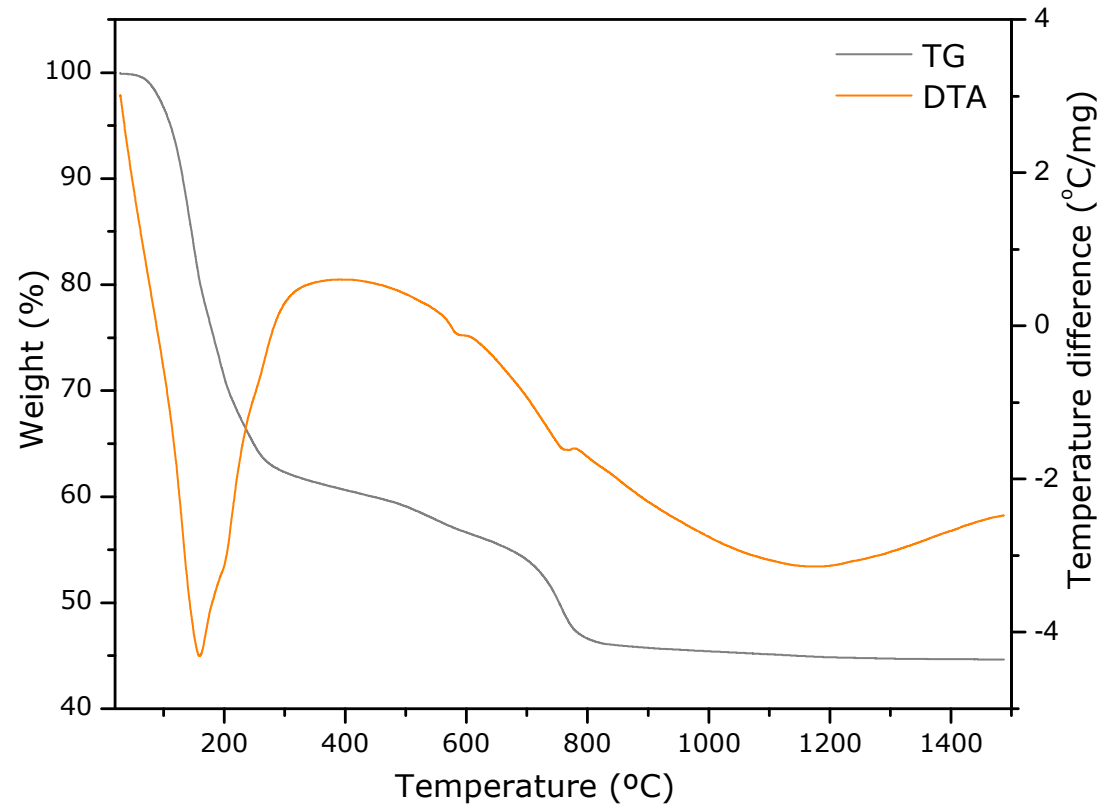

Figure 3. TGA and DTA curves of the SG-dried solid.

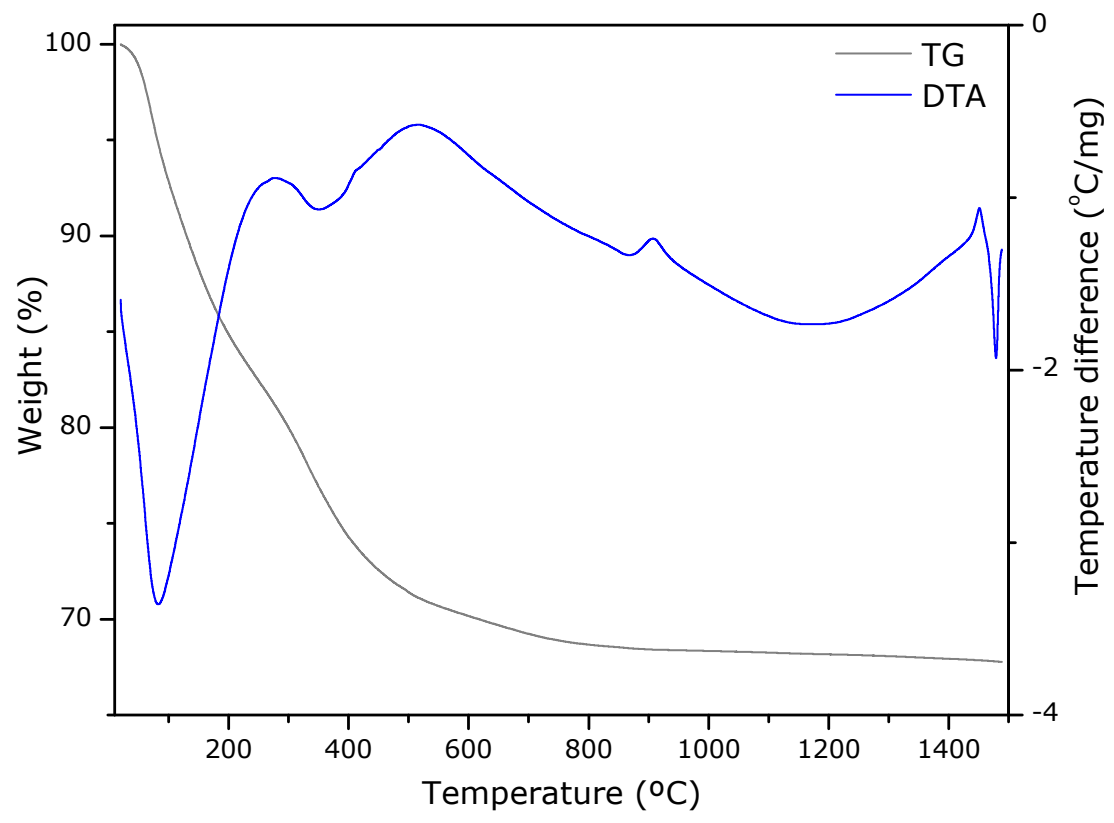

Figure 4. TGA and DTA curves of the CP-dried solid. 
The thermal curves of CP-dried sample are shown in Figure 4, being rather different than those from the sample prepared by sol-gel procedure. The initial weight loss, c.a. 7 wt. \%, is extended from room temperature to about $450{ }^{\circ} \mathrm{C}$, and it is composed of two processes, centred at about 100 and $390{ }^{\circ} \mathrm{C}$, associated to endothermal effects. As reported previously, this solid is mainly composed of Ni-Al hydrotalcite, and these effects are compatible with the thermal behaviour of hydrotalcite-like compounds. Thus, the first effect is due to the removal of interlayer water molecules and the second one is due to the removal of both the water generated from hydroxyl groups and of the carbon dioxide generated by the decomposition of the carbonate interlayer anions [18]. At the same time, water from amorphous silica may be removed in the first step of weight loss. After such processes, the solid may be composed by a mixture of oxides, as observed by X-ray diffraction, crystalline in the case of $\mathrm{NiO}$ and amorphous in the case of the other elements. As expected, no more weight change processes are observed at higher temperatures, but an exothermal effect is observed in the DTA curve at about $970{ }^{\circ} \mathrm{C}$. This effect may be assigned to the transformation from $\mathrm{NiO}$ and $\mathrm{Al}_{2} \mathrm{O}_{3}$ to $\mathrm{NiAl}_{2} \mathrm{O}_{4}$. As discussed previously, the solid calcined under static conditions at $1000{ }^{\circ} \mathrm{C}$ show the presence of a certain percentage of the spinel. Its formation implies the solid-phase reaction between crystalline $\mathrm{NiO}$ and amorphous $\mathrm{Al}_{2} \mathrm{O}_{3}$, with migration and re-ordering of the cations. It is remarkable that such an effect is not detected in the SG-dried solid, probably because of the nature of the oxides before the solid-state reaction.

\section{VII.3.4. UV-Vis spectroscopy}

The colour of the solids was found to be dependent on the synthetic method and on the temperature of calcination of each solid, varying between green and brownyellow colours. However, even at $1000{ }^{\circ} \mathrm{C}$ the characteristic blue colour of $\mathrm{NiAl}_{2} \mathrm{O}_{4}$ spinel was not observed, probably by the small amount of this phase in this solid, mainly composed of NiO. A detailed study of the UV-Vis spectra of the solids was carried out. The spectra of the SG series are given in Figure 5, those of the solids from CP series being very similar. 


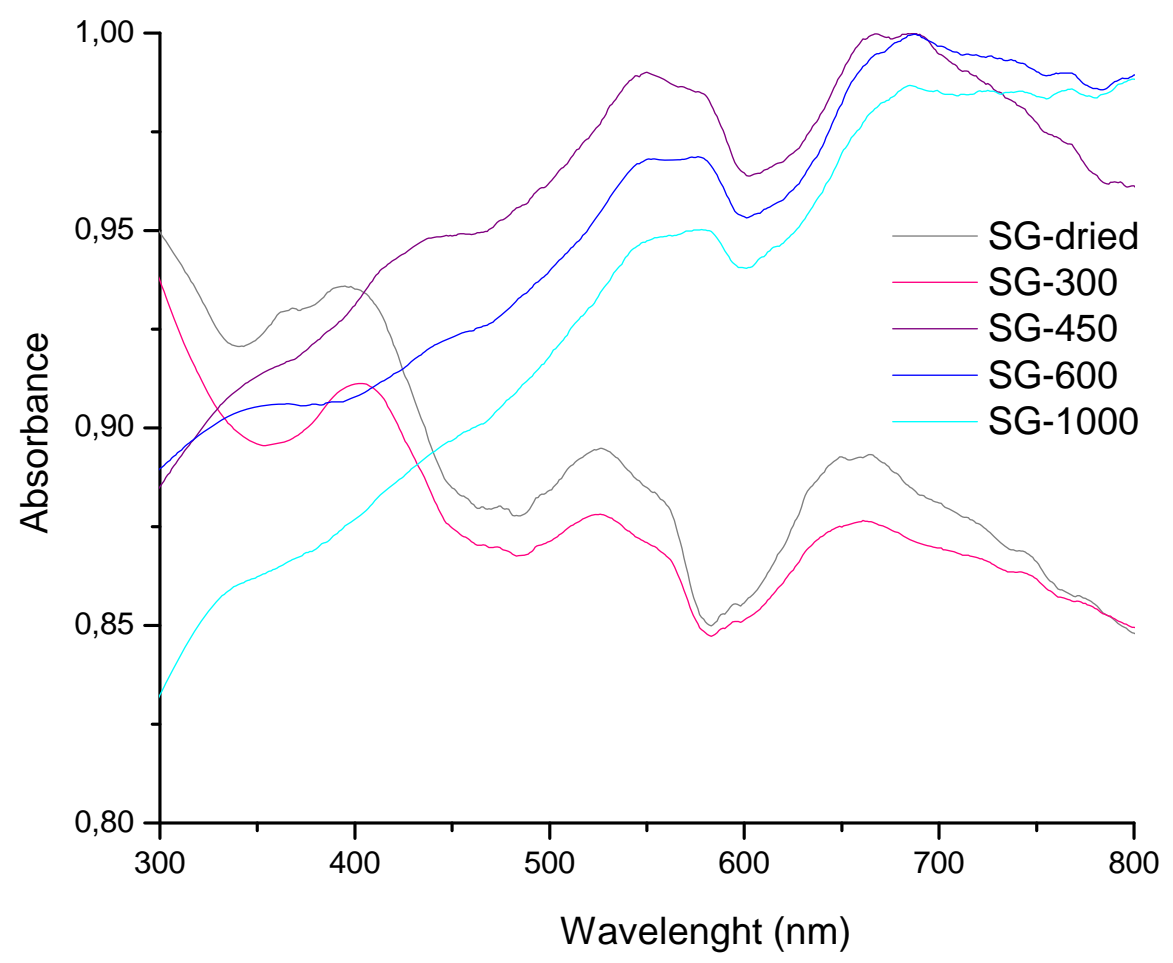

Figure 5. UV-Vis spectra of the SG series solids.

The only element that can produce the coloration of the solids is nickel. As it is well known, this element, with a $d^{8}$ electronic configuration, has three spin-allowed transitions when located in octahedral coordination, namely ${ }^{3} A_{2 g}(F) \rightarrow{ }^{3} T_{2 g}(F)$, ${ }^{3} A_{2 g}(F) \rightarrow{ }^{3} T_{1 g}(F)$ and ${ }^{3} A_{2 g}(F) \rightarrow{ }^{3} T_{1 g}(P)$, denoted $v_{1}, v_{2}$ and $v_{3}$, respectively. The position of the corresponding bands strongly depends in the nature of the ligands, appearing at 770-1400, 500-900 and 370-525 nm, respectively, which makes that most of times only two of the bands are observed in the visible region, the third one being in the NIR region, for low-field ligands, or in the UV region, for the highfield ligands. For $\mathrm{NiO}$, these bands have been reported at 1160, 740 and $405 \mathrm{~nm}$. Besides, two forbidden transitions can be observed, registered at 670 and $460 \mathrm{~nm}$ for $\mathrm{NiO}$, and the charge transference band uses to be very intense and to be located close to $220 \mathrm{~nm}$ [19].

All of our solids, prepared by both methods, show two bands in the visible region, corresponding to $v_{1}$ and $v_{2}$ transitions. In the sol-gel series, these effects are located close to 525 and $655 \mathrm{~nm}$, the last one actually appearing as two bands, 
centred at about 645 and $665 \mathrm{~nm}$, in the solids dried and calcined at $300{ }^{\circ} \mathrm{C}$, positions typical of $\mathrm{Ni}^{2+}$ in octahedral coordination [20]. These bands shift to c.a. 550 and $685 \mathrm{~nm}$ in the solids calcined at 450, 600 and $1000{ }^{\circ} \mathrm{C}$. This shifting is compatible with the transformation from phases close to hydroxides to phases close to oxides, that is, the coordination sphere of the cation passing from being majority occupied by hydroxyl to be occupied by oxo anions, this provoking a decrease in the field stabilization around the cations.

No significant changes were observed in the spectrum of the solid calcined at $1000{ }^{\circ} \mathrm{C}$, although $\mathrm{Ni}^{2+}$ in tetrahedral coordination exists in this phase and in spite of because the tetrahedral transitions are more intense than the octahedral ones, effects from cations in this environment may be expected. However, X-ray diffraction shows that this phase is minority even after calcination at this temperature, and besides only $1 / 4$ of $\mathrm{Ni}^{2+}$ cations in $\mathrm{NiAl}_{2} \mathrm{O}_{4}$ spinel are in tetrahedral coordination environment. Only a very small effect at $585 \mathrm{~nm}$ can be assigned to $\mathrm{Ni}^{2+}$ in spinel, which is in good agreement with the position described by Shen et al. [21] for this phase in NiW/alumina catalysts. The same can be said for the CP series, although in this case the band at $585 \mathrm{~nm}$ in the solid calcined at $1000{ }^{\circ} \mathrm{C}$ is more intense. No bands from $\mathrm{Ni}^{3+}$ were detected, in opposite to the results reported for $\mathrm{Ni}$-Al hydrotalcite calcined at several temperatures [22].

\section{VII.3.5. Textural properties}

The textural properties of the solids are summarized in Table 2. All the solids of the SG series were analysed, but only three solids in the CP series. All the solids in the SG series show low BET surface area values, which varies between $32 \mathrm{~m}^{2} / \mathrm{g}$ for the dried solid to $2 \mathrm{~m}^{2} / \mathrm{g}$ for the solid calcined at $1000{ }^{\circ} \mathrm{C}$. It is remarkable that the surface area decreases slowly when increasing the temperature, and thus the solid calcined at $600{ }^{\circ} \mathrm{C}$ show a surface area of $19 \mathrm{~m}^{2} / \mathrm{g}$, in fact higher than that of the solid calcined at $450{ }^{\circ} \mathrm{C}$. The decrease in the surface area may be easily related to the dehydroxilation of the solids. The low value of the surface area in the solid calcined at $450{ }^{\circ} \mathrm{C}$ may be related to the collapse of hydroxide phases above described and the presence of crystalline $\mathrm{NaCl}$. The disapparition of $\mathrm{NaCl}$ with incorporation of sodium to the amorphous silicoaluminate phases produces the increase in surface area observed when increasing the calcination temperature from 450 to $600^{\circ} \mathrm{C}$. 


\begin{tabular}{|c|c|c|c|c|}
\hline Sample & $\mathrm{S}_{\mathrm{BET}}^{\mathrm{a}}\left(\mathrm{m}^{2} / \mathrm{g}\right)$ & $\mathrm{S}_{\text {ext }}^{b}\left(m^{2} / g\right)$ & $V p^{c}\left(\mathrm{~cm}^{3} / g\right)$ & $V_{\mu} p_{t}{ }^{d}\left(\mathrm{~cm}^{3} / g\right)$ \\
\hline SG-blank & $11\left(C^{e}=64\right)$ & 5 & 0.014 & 0.007 \\
\hline SG-dried & $32(C=105)$ & 31 & 0.055 & 0.000 \\
\hline SG-300 & $26(C=108)$ & 25 & 0.049 & 0.000 \\
\hline SG-450 & $14(C=67)$ & 8 & 0.020 & 0.005 \\
\hline SG-600 & $19(C=101)$ & 16 & 0.045 & 0.001 \\
\hline SG-1000 & $2(C=196)$ & 0.2 & 0.003 & 0.001 \\
\hline CP-blank & $157(C=130)$ & 140 & 0.446 & 0.004 \\
\hline CP-dried & $302(C=111)$ & 297 & 0.608 & 0.000 \\
\hline CP-1000 & $65(C=108)$ & 60 & 0.176 & 0.002 \\
\hline
\end{tabular}

Table 2. Textural characteristics of the solids, obtained from the adsorption of nitrogen at $-196{ }^{\circ} \mathrm{C}$. ${ }^{a}$ Specific surface areas from the BET equation $\left(0.05 \leq \mathrm{p} / \mathrm{p}^{\circ}\right.$ $\leq 0.20$, interval of relative pressure). ${ }^{b}$ Specific external surface area obtained from the $t$ method. ${ }^{c}$ Specific total pore volumes at a relative pressure of $0.95 .{ }^{d}$ Specific micropore volumes derived from the $t$ method. ${ }^{e}$ BET C-values, characteristic of the intensity of the adsorbate-adsorbent interactions.

The same behaviour is observed for the pore volume, also given in Table 2, while the micropore surface area and micropore volume show in all cases very low values. The increase in the size of the $\mathrm{NiO}$ particles and sintering of the amorphous phase produce the decrease of surface area when calcining at $1000{ }^{\circ} \mathrm{C}$, as may be expected when heating to a so high temperature.

The dried solid prepared by the CP method shows a high surface area, $157 \mathrm{~m}^{2} / \mathrm{g}$, much higher than in the solid prepared by the SG method. This is a value even higher than those usually reported for hydrotalcite compounds. The surface area may be due to a contribution from the hydrotalcite-like phase, clearly detected by X-ray diffraction, and from the amorphous silica-alumina phase. Besides, the fact that the layers of the hydrotalcite phase are poorly ordered may increase the contribution from this phase, in agreement with the high contribution of the external surface area to the total area of this solid. It is remarkable that the surface area of the solid of this series calcined at $1000{ }^{\circ} \mathrm{C}$ shows a high value, $65 \mathrm{~m}^{2} / \mathrm{g}$, much higher than for hydrotalcites calcined at this temperature. $\mathrm{NiO}$ and 184 
$\mathrm{NiAl}_{2} \mathrm{O}_{4}$, even if the spinel is poorly crystalline, may contribute in a very limited extent to the surface area, which may be mainly due to the amorphous silicaalumina phase. This suggests that the sintering of silica-alumina phase is delayed by the presence of $\mathrm{Ni}$, and eventually of $\mathrm{Na}$, and makes more interesting the $\mathrm{NiO}$ $\mathrm{NiAl}_{2} \mathrm{O}_{4} /$ silica-alumina nanocomposite, which composes the solid calcined at $1000^{\circ} \mathrm{C}$.

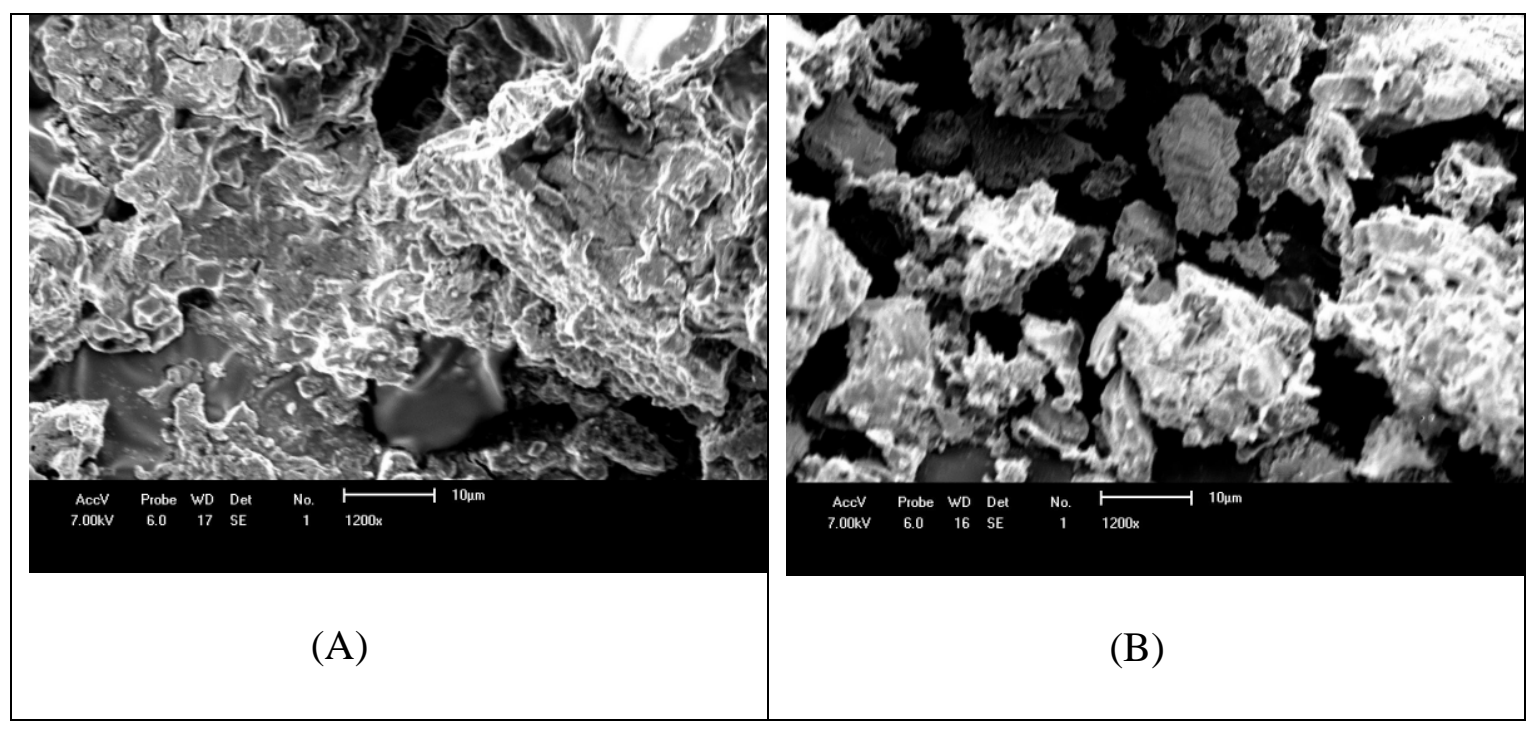

Figure 6. SEM images of: A) SG-dried; B) SG-450 solids.

Finally, scanning electron micrographs (see Figure 6) of the solids show the characteristic aspect of this type of solids, with spongy particles, even when the solids have low surface area values. In the calcined solids, this spongy habit gives rise to much more closed particles.

\section{VII.3.6. Catalytic performance}

In order to check the catalytic behaviour of the Ni-containing catalysts, the epoxidation of cyclooctene with $\mathrm{H}_{2} \mathrm{O}_{2}$ was studied at room temperature and atmospheric pressure. As indicated previously, (Z)-cyclooctene exclusively reacts by epoxidation, giving cyclooctene epoxide as only product (see Scheme I). In our case, $\mathrm{H}_{2} \mathrm{O}_{2}$ was chosen as oxygen source because it is a clean and environmentally friendly oxidant yet water and oxygen are the only products of its reaction. The concentration of $\mathrm{H}_{2} \mathrm{O}_{2}$ was 70 wt \%, following the results reported by Rinaldi et al. [23], who found that the use of this concentration improves the lifetime and the 
productivity of the catalysts, due to a smaller extent of $\mathrm{H}_{2} \mathrm{O}_{2}$ decomposition, allowing good epoxide yields with a reduced formation of molecular oxygen.

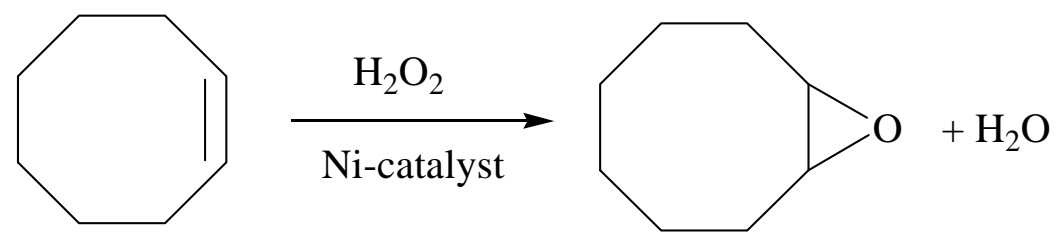

Scheme 1. Reaction of epoxidation of (Z)-cyclooctene with hydrogen peroxide.

Before beginning the reaction in the presence of the catalysts, control tests were carried out in the absence of oxidant, finding that they did not lead to any product yields.

It may be remarked that no lixiviation of the catalysts was observed, for any of the solids studied and under any of the conditions considered. This point was verified from the UV-Vis spectra of the supernatant solutions obtained after the oxidation reactions were completed. No bands of $\mathrm{Ni}^{2+}$ were detected in them. Obviously, this technique does not allow discarding leaching of other elements from the solids, such as $\mathrm{Al}^{3+}$, because they do not show any band in this region, but leaching of these elements is not expectable.

In order to verify the contribution of the homogeneous reaction, the solid catalysts were filtered off from the reaction medium after a reaction cycle of 24 hours, an additional amount of hydrogen peroxide was added to the resulting supernatant, and the resulting mixture was submitted to reaction for another 24 hours under the same initial conditions. It was observed that there was no catalytic activity, thus confirming the exclusively heterogeneous character of the catalytic process. It was also observed that a solution of $\mathrm{Ni}$ chloride produces the transformation of $25 \%$ of cyclooctene after 24 hours, $39 \%$ after 48 hours and $71 \%$ after 72 hours, thus again confirming the absence of $\mathrm{Ni}^{2+}$ leached from the catalysts in the supernatant, although as commented before, the possible leaching of other elements from the catalysts can not be discarded, but it may be affirmed that they form catalytically inactive species, as observed by Coles et al. [24] for titania-silica catalysts. 
The results obtained by using the catalysts from the SG series are shown in Figure 7. As indicated before, cyclooctene epoxide is the only product of the reaction, so the conversion of the reactant is the same that the yield to the product, and we may use these terms as synonymous. The conversion strongly varies with the temperature of calcination of the solids.

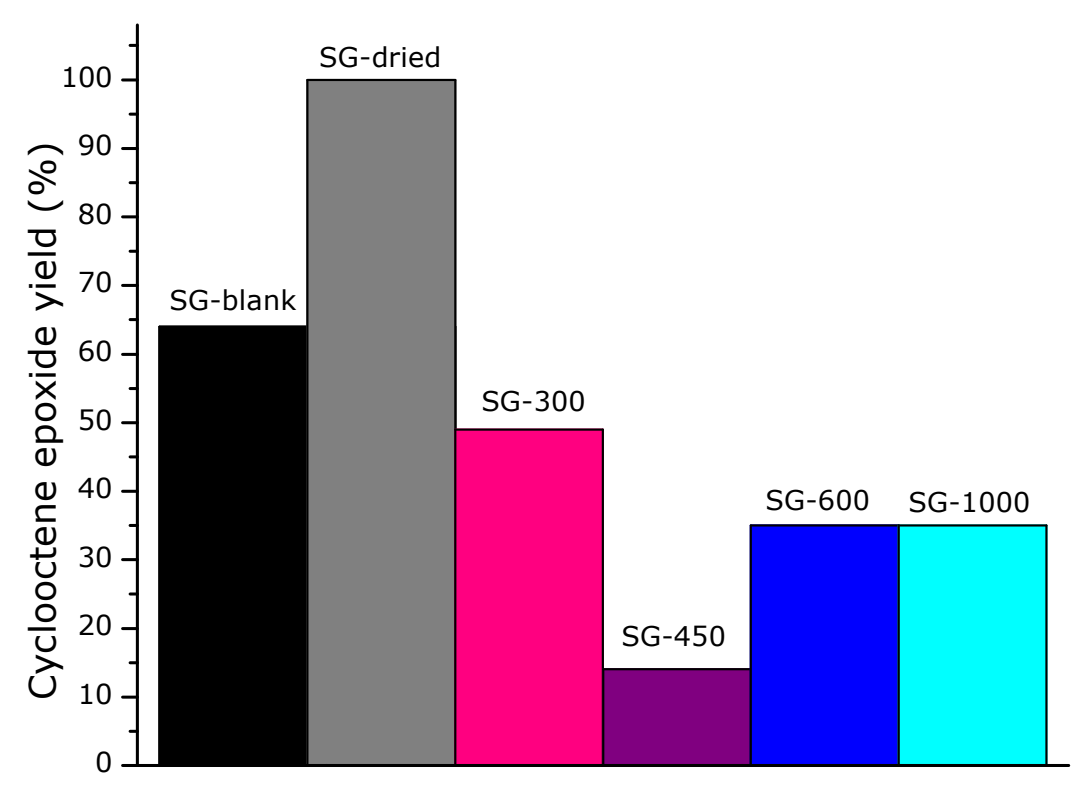

Figure 7. Catalytic results of SG solids in the oxidation of (Z)-cyclooctene using hydrogen peroxide as oxygen donor.

The SG-dried sample exhibits the highest performance, reaching a yield of cyclooctene epoxide of $100 \%$. This result may be explained by the presence of nickel in various forms, as previously reported by X-ray diffraction; it is well established in the literature that nickel can act as active species in the transfer of oxygen atoms from the oxidant to organic substrates [25]. Besides, the surface area and pore volume of this solid is relatively high, the higher in this series. In the same way, a certain number of layers are present in this solid, with a poor degree of ordering in the c-axis, so they are widely delaminated, both $\mathrm{Al}^{3+}$ and $\mathrm{Ni}^{2+}$ can be occupying the octahedral sites. This may make the approach of the olefin to these layers very easy, giving a good accessibility to the cations located in the octahedral positions. It is important to note that Rinaldi et al. [23] have reported that $\mathrm{Al}^{3+}$ is active for cyclooctene epoxidation, which may also explain the fact that the blank 
solid of this series, without nickel, shows a conversion of $64 \%$. These authors investigated the activity for this reaction of a free transition metal alumina prepared by sol-gel methodology, finding that it is an efficient heterogeneous catalyst. For this catalyst, the activation of hydrogen peroxide occurred probably through the reaction of weak acidic sites $(\mathrm{Al}-\mathrm{OH})$ of the alumina surface to form hydroperoxide groups, which are responsible for the transfer of oxygen to the (Z)-cyclooctene. This suggests a strong influence of both the acidity of the solids and of their textural properties. The results obtained for the blank solids, both in this series and in that prepared by co-precipitation (see below), together with the results reported by Rinaldi et al. [23], open up wide perspectives for the use of these type of materials as catalysts for the oxidation of olefins, since they are free of transition metal, being much less polluting materials.

Two different mechanisms have been proposed in the literature for oxidation reactions when transition metals are used as catalysts [25]. In the first one, the oxidant active species is ascribed as the high-valent oxo-metal species $\mathrm{Ni}^{\mathrm{IV}}=\mathrm{O}$ formed via a two-electron oxidation of the $\mathrm{Ni}^{2+}$ ions by hydrogen peroxide species; later the $\mathrm{Ni}^{\mathrm{IV}}=\mathrm{O}$ species transfer the oxygen atom to the epoxide carbon-carbon double bond, thus forming the epoxide. In the second mechanism, the oxidizing species are free radicals generated in the presence of metals and/or due to the homolytic cleavage of the $0-0$ hydrogen peroxide bond [25]. In the attempt to discover which active species is acting in the reaction between the SG materials and $\mathrm{H}_{2} \mathrm{O}_{2}$, we carried out the $(\mathrm{Z})$-cyclooctene epoxidation reactions again, in the presence of the radical scavenger tert-butyl alcohol $[10,26,27]$. This radical scavenger diminishes the product yields in $22 \%$, indicating that a contribution of radical mechanisms also exists in this system. It is remarkable that although the participation of a radical process is happening, the epoxide was detected as only product, with a total conversion.

SG-300 produces $49 \%$ of cyclooctene epoxide. Comparing to the previous solid, SG-300 still have a complex composition, with various nickel crystalline phases, and its specific surface area and pore volume show only a small decrease with respect to the values of these magnitudes in the dried solid. The silicate layers strongly collapse, the partial c-ordering observed in the dried sample completely 
disappears, and even the individual layers are altered. This fact may make the access of the substrate to the active sites more difficult, which results in a decrease of the catalytic activity.

The solid SG-450 shows a low conversion, only $14 \%$, the lowest in all the series. This result is logical, considering that this is the solid with a lower value of surface area in the series, and that although NiO crystals are already formed, their size is not very large. At the same time, crystalline $\mathrm{NaCl}$ composes the solid, but this compound seems to not contribute to the catalytic behaviour.

The trend changes in the two solids calcined at higher temperatures, both show a conversion of $35 \%$. It may seem contradictory that the activity is the same for two solids rather different, but this activity is the sum of two factors. The solid SG600 is composed of large crystals of $\mathrm{NiO}$ over amorphous silica-alumina, and $\mathrm{NiO}$ is capable to produce the active $\mathrm{Ni}^{\mathrm{IV}}=\mathrm{O}$ species. Besides, this solid still conserves a relatively high surface area, $19 \mathrm{~m}^{2} / \mathrm{g}$, and pore volume, $0.045 \mathrm{~cm}^{3} / \mathrm{g}$. On the other hand, solid SG-1000 shows surface area and pore volume values very close to zero, and besides of large crystals of $\mathrm{NiO}$, it is also composed of spinel, also over a silicaalumina amorphous phase and mainly this amorphous phase suffers the sintering, making difficult to access to Al cations. It is reasonable to expect that the strong deleterious of the textural properties will lead to a decrease in the catalytic activity, and this decrease seem to be compensated by the formation of the spinel.

The catalytic behaviour of the solids prepared by co-precipitation is shown in Figure 8. It can be seen that all of them catalyses epoxidation of (Z)-cyclooctene. However, some differences are observed with respect to the activity of the solids from the SG series. Thus, the catalytic activity of all the CP solids is almost constant, with values between $20-35 \%$, that is, the influence of the temperature of calcination is very low. The blank solid of this series shows a conversion of $40 \%$.

These results can again be discussed on the basis of the chemical composition of the solids and of their textural properties. The blank solid is completely amorphous, no layered phases are observed, which differentiates it from the blank solid of the SG method. The solids CP-dried, CP-300 and CP-450 are mainly composed of a hydrotalcite-like phase, silica being as an amorphous phase, and the 
catalytic results clearly suggest that the sites occupied for both $\mathrm{Ni}^{2+}$ and $\mathrm{Al}^{3+}$ in these solids make them much less active than in the corresponding SG series counterparts. It is remarkable that solids in CP series show much larger surface area values than those in SG series, and as observed, this is not a key factor conditioning the catalytic activity when comparing solids from both series, the nature of the sites seems to be much more important.

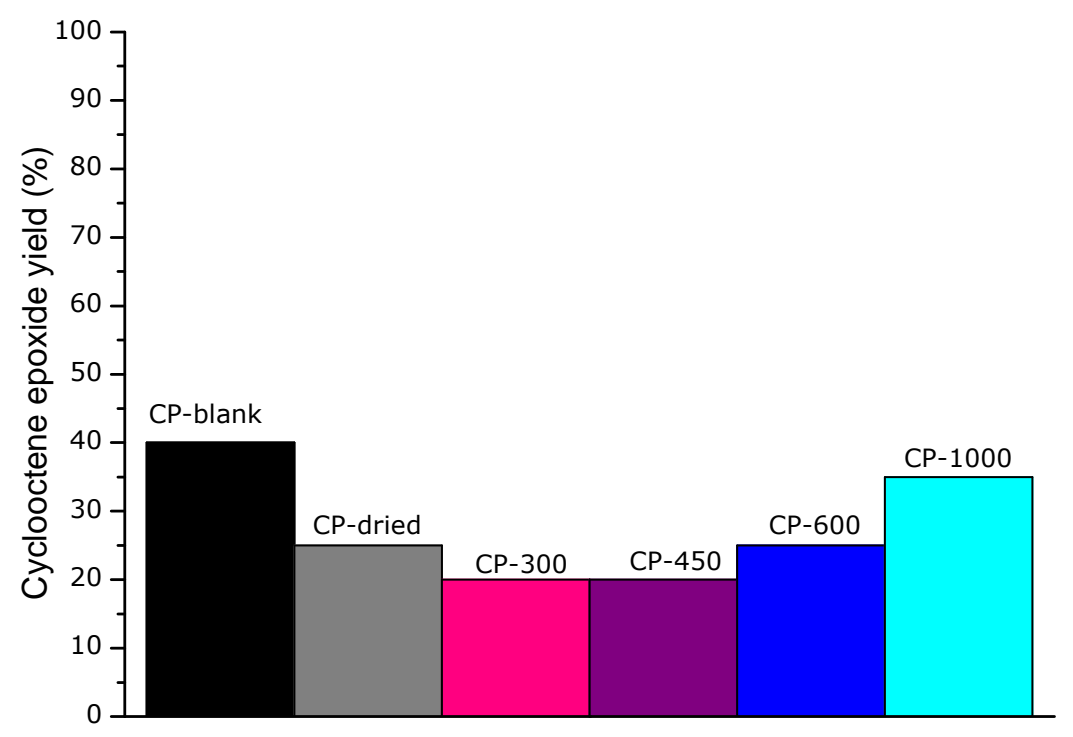

Figure 8. Catalytic results of CP solids in the oxidation of (Z)-cyclooctene using hydrogen peroxide as oxygen donor.

A steric factor may also be taken into account. Layers of hydrotalcite are relatively well ordered, and the basal spacing of this compound is $7.76 \AA$, which considering the height of each sheet gives an interlayer spacing of c.a. $3 \AA$, the accessibility of the reactants to the interlayer spacing being rather difficult. The acidity of the solids may also be taken into account, particularly in the case of synergic effects. It is clear that in the CP solids, silica is forming the amorphous phase while $\mathrm{Ni}$ and $\mathrm{Al}$ are confined forming the hydrotalcite. Even if the silica phase is rather acidic, acid and metal sites are enough separated to produce synergic effects. However, in the solids prepared by the SG method, both types of active centres are much close, particularly because $\mathrm{Al}^{3+}$ is forming the silica-alumina amorphous phase. 
Solids CP-600 and CP-1000 show exactly the same catalytic activity that the SG solids calcined at the same temperatures. As textural parameters are rather different, it seems evident that the activity is controlled by the presence of $\mathrm{NiO}$ and $\mathrm{NiAl}_{2} \mathrm{O}_{4}$ crystals, very similar in the solids from both series, giving rise to the same catalytic activity.

As in the SG series, we carried out the (Z)-cyclooctene epoxidation reaction over the $\mathrm{CP}$ solids in presence of the radical scavenger tert-butyl alcohol, the presence of the radical scavenger surprisingly increased the epoxide yields in almost $100 \%$, confirming what was observed in the SG series, the existence of the radical process, and the strong competition between the $\mathrm{Ni}^{\mathrm{IV}}=\mathrm{O}$ and the radical species, being remarkable that for the $\mathrm{CP}$ series solids the radical mechanism controls the process. The acid character of the CP solids seems to trigger the radical process favouring the formation of radicals species and when these species are trapped by the radical scavenger the only species that acts is $\mathrm{Ni}^{\mathrm{iV}}=\mathrm{O}$, without competition. It is worth to point out that the mechanism for the oxidation of alkenes with hydrogen peroxide involving nickel ions is still under strong controversy, and due to this fact, additional experiments will be accomplished with selected solids between those prepared in this work.

\section{VII.4. Conclusions}

Two series of samples were prepared by reaction of $\mathrm{Si}, \mathrm{Al}, \mathrm{Ni}$ and $\mathrm{Na}$ precursors by sol-gel and co-precipitation methods. Although the conditions were chosen to obtain layered smectitic clay saponite, only individual layers of this clay were obtained by the first method, together with a complex mixture of compounds, while $\mathrm{Ni}-\mathrm{Al}$ hydrotalcite, takovite, together with amorphous silica, was obtained by the second method. Thus, much effort may be done to optimise the obtaining of saponite. However, the solids obtained, and those obtained by their calcination at various temperatures, which may be seen as $\mathrm{NiO}$ or $\mathrm{NiAl}_{2} \mathrm{O}_{4}$ over amorphous silicaalumina phases, show interesting properties as catalysts. Their oxidation activity was tested for the epoxidation of $(\mathrm{Z})$-cyclooctane with $\mathrm{H}_{2} \mathrm{O}_{2}$, under environmental friendly conditions. Very different results were obtained for the various solids, in some cases reaching $100 \%$ conversion of the cycloalkene, the corresponding 
epoxide being the only product detected. However, some questions rise from our results, such as the influence of radical mechanism and the surface acid-base properties of the solids in the reaction.

\section{VII.5. References}

[1] G.W. Brindley, G. Brown. Crystal Structures of Clay Minerals and their X-Ray Identification; Mineralogical Society, London, U.K. 1984, 495 pp.

[2] M.A. Vicente Rodríguez, M. Suárez Barrios, J.D. López González, M.A. BañaresMuñoz, Clays Clay Miner. 42 (1994) 724.

[3] J.F. Lambert, G. Poncelet, Topics Catal. 4 (1997) 43.

[4] L.J. Michot, F. Villiéras, J.F. Lambert, L. Bergaoui, Y. Grillet, J.L. Robert, J. Phys. Chem. B 102 (1998) 3466.

[5] L. Bergaoui, J.F. Lambert, M.A. Vicente Rodríguez, L.J. Michot, F. Villiéras, Langmuir 11 (1995) 2849.

[6] J.T. Lutz, Jr., in: Kirk-Othmer, M. Grayson, D. Eckroth, G.J. Bushey, C.I. Eastman, A. Klingsberg, L. Spiro (Eds.), Encyclopedia of Chemical Technology, Vol. 9, 3rd Edition, Wiley, New York, 1980, pp 251-266.

[7] D.E. De Vos, B.F. Sels, P.A. Jacobs, Adv. Synth. Catal. 345 (2003) 457.

[8] B.S. Lane, K. Burgess, Chem. Rev. 103 (2003) 2457.

[9] S.H. Jhung, J.-H. Lee, A.K. Cheetham, G. Férey, J.-S. Chang, J. Catal. 239 (2006) 97.

[10] B.L. Caetano, L.A. Rocha, E. Molina, Z.N. Rocha, G. Ricci, P.C. Calefi, O.J. de Lima, E.J. Nassar, K.J. Ciuffi, Appl. Catal. A: 311 (2006) 122.

[11] O.J. de Lima, D.P. de Aguirre, D.C. de Oliveira, M.A. da Silva, C. Mello, C.A.P. Leite, H.C. Sacco, K.J. Ciuffi, J. Mater. Chem.11 (2001) 2476.

[12] JCPDS File, Joint Committee on Powder Diffraction Standards, International Centre for Diffraction Data, Philadelphia, 1977.

[13] N.N. Greenwood and A. Earnshaw, Chemistry of the Elements, Second edition. Butterworth-Heinemann, Elsevier Science, 1997.

[14] H. Suquet, C. de la Calle, H. Pezerat, Clays Clay Miner. 23 (1975) 1.

[15] V. Rives, M.A. Ulibarri, Coord. Chem. Rev. 181 (1999) 61.

[16] Layered Double Hydroxides: Present and Future. V Rives (Ed.) Nova Sci. Pub. Inc., New York, 2001.

[17] D.L. Bish, G.W. Brindley, Am. Mineral. 62 (1977) 458.

[18] M.J. Holgado, V. Rives, M.S. San Román, Appl. Catal. A 214 (2001) 219. 
[19] A.B.P. Lever. Inorganic Electronic Spectroscopy. Monograph 1 in M.F. Lappert (Ed.) Physical Inorganic Chemistry. Elsevier Publishing Company, Amsterdam, 1968.

[20] V. Rives, App. Catal. A 214 (2001) 219.

[21] B. Shen, H. Li, W. Zhang, Y. Zhao, Z. Zhang, X. Wang and S. Shen, Catal. Today 106 (2005) 206.

[22] F. Prinetto, D. Tichit, R. Teissier, B. Coq, Catal. Today 55 (2000) 103.

[23] R. Rinaldi, J. Sepúlveda, U. Schuchardt, Adv. Synth. Catal. 346 (2004) 281.

[24] M.P. Coles, C.G. Lugmair, K.W. Terry, T.D. Tilley, Chem. Mater. 12 (2000) 122.

[25] D. Lee, H. Bang and M.P. Suh, J. Mol. Catal. A 151 (2000) 71.

[26] J.S. Daniels, K.S. Gates, J. Am. Chem. Soc. 118 (1996) 3380.

[27] T. Punniyamurthy, M.M. Reddy, S.J.S. Kalra, J. Iqbal, Pure Appl. Chem. 68 (1996) 619. 

Capítulo VI I I

Conclusiones 

En la presente Tesis Doctoral se han preparado catalizadores basados en materiales arcillosos y en dos elementos de transición catalíticamente activos, cromo y níquel. Se pretende aprovechar las propiedades singulares de los materiales arcillosos, muy apropiadas para su utilización como soportes catalíticos, y combinarlas con las propiedades catalíticas de los metales. En la mayoría de los casos, se han utilizado arcillas de origen natural, que han sido modificadas en el laboratorio por diversos métodos, aunque en algunas ocasiones se han utilizado materiales sintéticos.

En este último apartado se recopilan los resultados y las conclusiones más relevantes de cada uno de los capítulos anteriores.

\section{VIII.1. Materiales de partida.}

Los materiales de partida utilizados en el presente trabajo son arcillas de origen natural, en concreto saponitas, procedentes del yacimiento de Yunclillos-Cabañas (Toledo). Esta materia prima presenta, entre otras ventajas, la utilización de nuestros propios recursos y tener un coste muy bajo, ya que las arcillas están presentes en la naturaleza en gran cantidad.

Las conclusiones más importantes sobre los materiales de partida utilizados son las siguientes:

1. Las arcillas suministradas por la empresa explotadora de los yacimientos son de alta pureza. No obstante, se purificaron mediante suspensión en agua, decantación y extracción de la fracción inferior a dos micras. Esta purificación permite la casi total eliminación de las impurezas mineralógicas, obteniéndose materiales de pureza muy elevada. Esto permitirá una mejor justificación de los resultados obtenidos, así como su comparación con resultados bibliográficos.

2. Las saponitas utilizadas tienen una elevada carga, mayoritariamente localizada sobre su capa tetraédrica. Esto hace que sean idóneas para su 
intercalación con policationes, y que presenten una elevada acidez que, junto a sus elevadas superficies específicas, las hacen óptimas para su utilización en la preparación de catalizadores. Por otra parte, contienen pocos cationes de transición, lo que favorece el estudio de su actividad catalítica, ya que sus cationes constituyentes no interfieren significativamente con el cromo y el níquel en su actividad en reacciones redox.

3. Por las propiedades antes mencionadas, se intentó la síntesis de una saponita que contenga $\mathrm{Ni}^{2+}$ como catión octaédrico (no se intentó en el caso del cromo, al no haberse encontrado referencia alguna al respecto, y no ser previsible que ningún catión de este elemento ocupe la capa octaédrica arcillosa). La obtención no fue exitosa, ni al utilizar el método sol-gel ni al utilizar el de co-precipitación. No obstante, se obtuvieron nanocompuestos de níquel soportados sobre aluminosilicatos, con propiedades interesantes, por lo que han sido ampliamente estudiados y utilizados.

\section{VIII.2. Propiedades de los catalizadores utilizados.}

Los catalizadores utilizados se han preparado por modificación química de las arcillas naturales indicadas en el apartado anterior. También se han utilizado los sólidos obtenidos en el laboratorio mediante los métodos sol-gel y co-precipitación. A continuación se indican las propiedades más importantes de los sólidos preparados en cada una de las series consideradas.

1. El proceso de intercalación de las saponitas con policationes de Keggin da como resultado la obtención de sólidos con un espaciado interlaminar muy elevado, que alcanza los 18,5 A. Esta intercalación se traduce en una mejora muy importante de las propiedades superficiales de la arcilla, principalmente su área superficial y su superficie de microporos.

2. En el caso de la intercalación con policationes mixtos $\mathrm{Al} / \mathrm{Cr}$, el proceso resulta igualmente exitoso, intercalándose policationes de Keggin de aluminio y especies de cromo con diferentes grados de polimerización, que 
van desde el monómero hasta los tetrámeros en función de las condiciones utilizadas para la preparación de los policationes, principalmente de la relación $\mathrm{Al} / \mathrm{Cr}$. Variando la relación $\mathrm{Al} / \mathrm{Cr}$ de las disoluciones intercalantes puede, además, controlarse el contenido en cromo de los sólidos finales.

3. Los sólidos intercalados presentan superficies específicas muy elevadas, la mayor contribución a esa superficie viene dada por el desarrollo de una estructura microporosa bidimensional generada por la introducción de policationes en el espacio interlaminar. Tras el proceso de calcinación, los sólidos pilareados muestran diferentes propiedades superficiales; se mantiene la estructura microporosa en el caso de los sólidos pilareados únicamente con aluminio o con aluminio y cantidades bajas de cromo, mientras que los sólidos con mayor contenido en cromo desarrollan superficie externa.

4. Los procesos de calcinación afectan de forma diferente a cada uno de los sólidos cointercalados en función de sus contenidos de aluminio y cromo, observándose que los catalizadores con un mayor contenido en cromo son menos estables a altas temperaturas que los intercalados sólo con policationes de aluminio, mientras que los sólidos que contienen simultáneamente $\mathrm{Al}$ y $\mathrm{Cr}$ conjugan las propiedades de ambos, la estabilidad térmica generada por los policationes de aluminio y las propiedades redox aportadas por la presencia de cromo.

5. La saponita previamente pilareada con policationes de aluminio es un excelente soporte para la preparación de los catalizadores soportados, ya que las propiedades superficiales de la arcilla natural se ven notablemente mejoradas mediante este tratamiento. La impregnación de este soporte con los precursores considerados es sencilla, no presentando ninguna dificultad reseñable.

6. La impregnación de la saponita pilareada permite obtener sólidos con contenidos controlados de cromo y níquel. Los sólidos impregnados secos presentan superficies específicas muy bajas, debido a que los precursores 
utilizados para la impregnación producen la oclusión de los poros del soporte, pero su posterior calcinación hace que los precursores se descompongan y los catalizadores finales presenten elevados valores de superficie específica y microporosidad.

7. Los catalizadores obtenidos tanto mediante pilarización como impregnación no presentan picos de difracción correspondientes a óxidos, oxihidróxidos o hidróxidos de los metales correspondientes, lo que indica el alto grado de dispersión de los metales sobre los soportes utilizados.

8. Al utilizar los métodos de preparación sol-gel y co-precipitación, se obtienen sólidos con diferencias muy notables tanto en su composición como en sus características. Aunque la obtención de saponita mediante estos métodos no resulta efectiva, al no orientarse las láminas arcillosas según el eje c, se obtienen sólidos cuyas composiciones y características superficiales pueden ser controladas.

9. Varios compuestos de níquel precipitan en las condiciones de preparación utilizadas en el método sol-gel, mientras mediante el método de coprecipitación sólo se obtienen sílice amorfa y una fase $\mathrm{Ni}$-Al tipo hidrotalcita (takovita). En ambos casos, las fases cristalinas varían notablemente al calcinar los sólidos a temperaturas crecientes, resultando mezclas de $\mathrm{NiO}$ y $\mathrm{NiAl}_{2} \mathrm{O}_{4}$ soportadas sobre fases de sílice y alúmina amorfas. Los sólidos obtenidos mediante ambos métodos contienen cantidades de níquel muy altas, entre el 38 y el $44 \%$, y la principal diferencia entre ellos es la ausencia de sodio en los catalizadores preparados mediante coprecipitación.

10. Las propiedades superficiales de estos sólidos son también muy diferentes en función del método de preparación utilizado. Los sólidos obtenidos mediante sol-gel presentan superficies bajas, que disminuyen al aumentar la temperatura utilizada en el tratamiento térmico. Sin embargo, el método de co-precipitación da lugar a sólidos con elevadas superficies específicas. 


\section{VIII.3. Reacciones catalíticas}

Los catalizadores metal/arcilla permiten combinar los elevados valores de área superficial y de porosidad de las arcillas con las propiedades catalíticas de los elementos metálicos incorporados. Los catalizadores basados en arcillas modificadas y fases activas de cromo o níquel son utilizados en reacciones de interés medioambiental; por un lado en la reducción de óxidos de nitrógeno, utilizando diversas mezclas oxidantes y, por otro lado, en la oxidación de hidrocarburos, en concreto, en la obtención de epóxido a partir de cicloocteno y en la oxidación de ciclohexano a ciclohexanol / ciclohexanona. La reducción de $\mathrm{NO}_{\mathrm{x}}$ elimina especies muy contaminantes, mientras que la oxidación de hidrocarburos constituye una alternativa limpia a los procesos industriales habituales, muy contaminantes.

Como en los apartados anteriores, a continuación se recogen las conclusiones más importantes de la utilización de los catalizadores en cada una de estas reacciones.

\section{VIII.3.1. Eliminación de óxidos de nitrógeno}

1. Los catalizadores cromo/arcilla presentan una actividad relativamente baja frente a la reducción de óxidos de nitrógeno, pero muy alta para la oxidación del propeno, hidrocarburo utilizado como reductor. La saponita pilareada presenta conversiones muy bajas, incluso a temperaturas elevadas, lo que indica que la actividad catalítica de los sólidos se debe a la incorporación de cromo.

2. La incorporación a los catalizadores de cantidades de cromo relativamente bajas es suficiente para llevar a cabo la oxidación del hidrocarburo. Cantidades por encima del $8 \%$ no producen una mejora de las conversiones.

3. Al estudiar la reacción entre el propeno y el oxígeno, se encuentran altas conversiones de hidrocarburo desde temperaturas de reacción intermedias, y los resultados son independientes de la forma de incorporación del cromo 
a los catalizadores. Pero al utilizar NO como oxidante, se encuentran conversiones diferentes para el oxidante y el reductor, sugiriendo que los catalizadores aportan oxígeno para la oxidación del hidrocarburo por un mecanismo tipo Mars-van Krevelen.

Al utilizar conjuntamente ambos oxidantes, $\mathrm{O}_{2}$ y $\mathrm{NO}$, se observa que las conversiones de propeno son mayores que las obtenidas cuando se utiliza únicamente NO, por lo que la reacción se ve favorecida por la mezcla de oxidantes. En todos los casos, las conversiones de NO son iguales al rendimiento a nitrógeno, sin que se observe $\mathrm{NH}_{3}$ ni otros compuestos nitrogenados en los productos de reacción.

4. Al utilizar los catalizadores basados en cromo, a temperaturas intermedias el aumento de oxígeno en el reactor favorece la oxidación de propeno sin que se observe una disminución de las conversiones de óxidos de nitrógeno, lo que evidencia un efecto sinérgico entre los oxidantes. Sin embargo, a temperaturas superiores la mezcla de oxidantes entra en competencia por la oxidación del hidrocarburo, lo que se traduce en una menor conversión de los óxidos de nitrógeno ya que el propeno reacciona preferentemente con el oxígeno. Todos los catalizadores de cromo tienen el mismo comportamiento, independientemente de la forma de incorporación del metal.

5. En el caso de los catalizadores de níquel, las conversiones de propeno a temperaturas intermedias son mayores para la mezcla de oxidantes que para la oxidación con oxígeno puro. A las máximas temperaturas estudiadas, la conversión del hidrocarburo se ve favorecida cuando el oxígeno es el único oxidante en la reacción.

6. Los catalizadores basados en níquel presentan distintas conversiones según el precursor utilizado en su preparación. En algunos casos, se detecta monóxido de carbono en los productos de reacción. Es decir, hay una importante influencia de los precursores utilizados en la actividad catalítica final, probablemente originada durante la descomposición de los contraiones de las sales precursoras. 


\section{VIII.3.2. Oxidación de cicloocteno y ciclohexano}

1. Al realizar pruebas "en blanco" ( $\sin$ catalizador, o con catalizador pero sin oxidante) se observan conversiones nulas, por lo que no hay reacción en fase homogénea.

2. Los catalizadores obtenidos por el método sol-gel presentan conversiones de cicloocteno que varían entre el 14 y el 100\%, con selectividad total hacia el epóxido. La conversión depende de la temperatura de calcinación, por lo que se ve influida por las propiedades superficiales de los catalizadores, la superficie específica, el tamaño de poro y las distintas fases formadas. No se detectó lixiviación de los catalizadores.

3. Los catalizadores obtenidos mediante co-precipitación dan lugar a conversiones de cicloocteno entre el 20 y el 35\%, también con selectividad total hacia el epóxido. No se observa una dependencia de la conversión respecto a la temperatura de preparación de cada sólido, aunque sí con su composición y sus propiedades superficiales. En esta serie, tampoco se detectó lixiviación de los catalizadores.

4. La comparación entre las actividades de los catalizadores obtenidos por ambos métodos sugiere una fuerte dependencia de la accesibilidad de los cationes $\mathrm{Ni}^{2+}$ y $\mathrm{Al}^{3+}$.

5. En la oxidación de ciclohexano sobre catalizadores $\mathrm{Ni}$ /arcilla, la conversión alcanzó valores del 28\% (4\% al utilizar la arcilla, sin níquel), siendo la selectividad total a ciclohexanona. El hecho de que no se forme ciclohexanol, junto con otras pruebas realizadas, indica que el ciclohexano es directamente oxidado a ciclohexanona, en una sola etapa, sin pasar por el alcohol. No se detectó lixiviación de los catalizadores.

6. La reacción fue estudiada en presencia de secuestradores de radicales, y los resultados obtenidos indican que la transferencia de oxígeno de los oxidantes (yodosilbenceno y peróxido de hidrógeno) a los sustratos es 
llevada a cabo por especies de níquel de alta valencia. Esto, junto con la selectividad total hacia un único producto, supone una fuerte similitud de estos catalizadores con sistemas biológicos enzimáticos, tales como el citocromo P-450, abriendo nuevas perspectivas a la utilización de este tipo de catalizadores en reacciones de oxidación. 